\title{
INFLUENCE OF MACH NUMBER ON PROFILE LOSS OF AXIAL-FLOW TURBINE BLADES
}

by

\author{
Mitch D. Kibsey
}

A Thesis submitted to the Faculty of Graduate Studies and Research in partial fulfilment of the requirements for the degree of

\section{Master of Applied Science}

in

Aerospace Engineering

\author{
Ottawa-Carleton Institute for \\ Mechanical \& Aerospace Engineering \\ Department of Mechanical and Aerospace Engineering \\ Carleton University \\ Ottawa, Ontario
}

(C) 2015

Mitch D. Kibsey 
The undersigned recommend to

the Faculty of Graduate Studies and Postdoctoral Affairs

acceptance of the thesis entitled

Influence of Mach Number on Profile Loss of Axial-Flow Turbine Blades

submitted by

Mitch D. Kibsey

in partial fulfilment of the requirements for the degree of

Master of Applied Science in Aerospace Engineering

Thesis Supervisor:

Professor Steen A. Sjolander

Department of Mechanical \& Aerospace Engineering

Department Chair:

Professor Metin I. Yaras

Department of Mechanical \& Aerospace Engineering

Carleton University 


\begin{abstract}
The current profile loss prediction methods for axial turbine blades predict a monotonic increase in profile losses at outlet Mach numbers above 1.0, while linear cascade testing in the literature has revealed a more complex behaviour. An objective of this investigation was to help clarify the flow features that are most influential on the profile losses in the transonic and supersonic regimes. Four linear cascades of turbine blades were investigated both experimentally and computationally. Linear cascade measurements were carried out using the High-Speed Wind Tunnel (HSWT) at Carleton University. It was found that the base pressure was strongly linked to the profile losses in the transonic and supersonic regimes. The position of the trailing edge shock impingement on the adjacent blade and its associated effect on the boundary layer were also found to influence the profile losses.
\end{abstract}




\section{Acknowledgments}

First and foremost, I wish to thank my thesis supervisor, Professor Steen A. Sjolander, for his invaluable guidance during the entire course of this project. His judicious and sound advice were essential to my understanding of the flow physics that were observed. Our discussions always brought to me a renewed enthusiasm for the project.

I wish to thank Hamza Abo El Ella, Steven Hall, Chris Sooriyakumaran and Gordon MacIsaac for kindly taking me under their wings and introducing me to wind tunnel operation at Carleton University. They willingly passed on their knowledge and experience, and patiently answered my many questions. I also greatly appreciate the suggestions of Andrew Scribner throughout the course of this research.

I wish to thank my colleagues Adrian Gerber, Mark Sutherland and Derek Ancrum for lending an ear when I was troubled or stuck, and for our much-needed lunch breaks. My Masters degree at Carleton was truly enjoyable in your company.

The financial support offered by the Ontario Student Assistance Program (OSAP - OGS), by the Natural Sciences and Engineering Research Council (NSERC), by Siemens and by Carleton University is gratefully acknowledged.

Lastly, I am tremendously grateful to my family for their unwavering and loving support throughout my academic endeavours. 


\section{Table of Contents}

Abstract $\quad$ iii

\begin{tabular}{|ll}
\hline Acknowledgments & iv
\end{tabular}

$\begin{array}{ll}\text { List of Tables } & \text { ix }\end{array}$

List of Figures $\quad x$

List of Symbols $\quad$ xvi

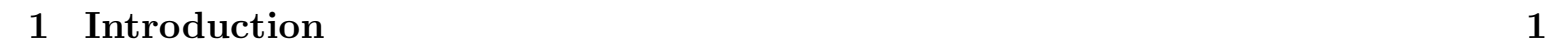

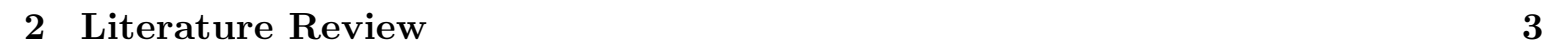

2.1 Introduction . . . . . . . . . . . . . . . . . . . . . 3

$2.2 \quad$ Flow Features Within an Axial Turbine Blade Row . . . . . . . . . . . . . . . 3

2.3 Loss Components Within an Axial Turbine Blade Row . . . . . . . . . . . . . . 5

2.4 Loss Coefficient Definitions $\ldots \ldots \ldots \ldots \ldots \ldots$

$2.5 \quad$ Aerodynamic Influences on Midspan Losses $\ldots \ldots \ldots$. . . . . . . . . . . . . . 8

2.5 .1 Mach Number . . . . . . . . . . . . . . . . . . . . . . . . . 8

$2.5 .2 \quad$ Base Pressure . . . . . . . . . . . . . . . . . . . . . . . . . . . . . . . 14

2.5 .3 Reynolds Number $\ldots \ldots \ldots \ldots$. . . . . . . . . . . . . . . . . . 19

2.5 .4 Freestream Turbulence $\ldots \ldots \ldots \ldots$. . . . . . . . . . . . 20

$2.5 .5 \quad$ Axial Velocity Density Ratio (AVDR) . . . . . . . . . . . . . . . 22

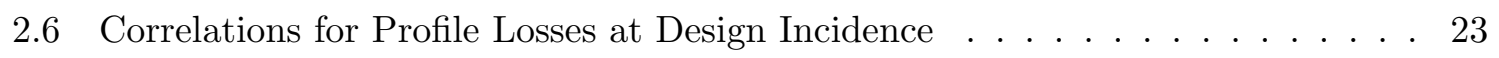




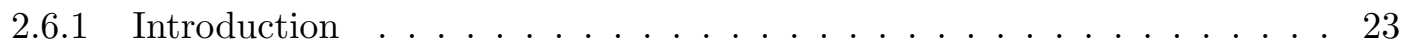

2.6 .2 Ainley and Mathieson Loss Correlation $\ldots \ldots \ldots \ldots \ldots$

2.6 .3 Dunham and Came Loss Correlation . . . . . . . . . . . . . 26

$2.6 .4 \quad$ Kacker and Okapuu Loss Correlation $\ldots \ldots \ldots \ldots \ldots \ldots$

2.6.5 Alternatives to the Ainley and Mathieson Family of Loss Correlations . 32

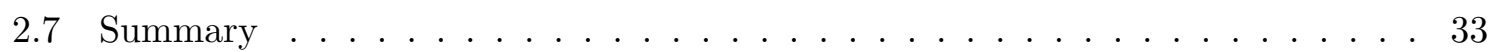

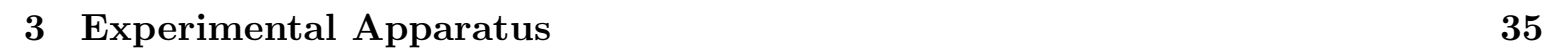

3.1 Introduction . . . . . . . . . . . . . . . . . . . 35

3.2 Pratt \& Whitney Canada High-Speed Wind Tunnel $\ldots \ldots \ldots \ldots$

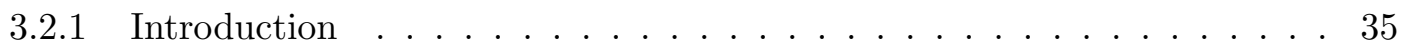

3.2 .2 Compressor $\ldots \ldots \ldots \ldots \ldots \ldots \ldots \ldots \ldots \ldots \ldots \ldots$

$3.2 .3 \quad$ Air Dryer $\ldots \ldots \ldots \ldots \ldots \ldots \ldots \ldots \ldots \ldots$

$3.2 .4 \quad$ Air Storage Tanks $\ldots \ldots \ldots \ldots \ldots \ldots$

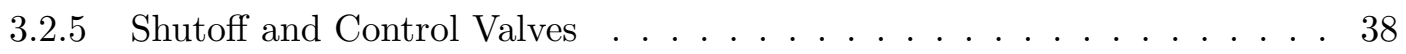

$3.2 .6 \quad$ Cascade Test Section . . . . . . . . . . . . . . . . . . . . . . 39

3.3 Wind Tunnel Instrumentation $\ldots \ldots \ldots$. . . . . . . . . . . . . 41

3.3 .1 Pressure Transducers . . . . . . . . . . . . . . . . . . . . . . . 41

$3.3 .2 \quad$ Upstream Reference Total Pressure and Temperature Sensors . . . . . . 42

3.3 .3 Pressure Measuring Probes $\ldots \ldots \ldots$. . . . . . . . . . . . . . 43

3.3 .4 Probe Traverse Mechanism . . . . . . . . . . . . . . . 45

3.4 Cascade Geometry . . . . . . . . . . . . . . . . . . . . 47

3.4 .1 AF4 Turbine Cascade . . . . . . . . . . . . . . . . 47

$3.4 .2 \quad$ HS1 Turbine Cascade Family $\ldots \ldots \ldots \ldots \ldots \ldots \ldots$

4 Experimental Procedures and Measurement Uncertainty $\quad 52$

4.1 Introduction . . . . . . . . . . . . . . . . . . . . . . . . 52

4.2 Control System $\ldots \ldots \ldots \ldots \ldots \ldots$

4.3 Data Acquisition Procedures $\ldots \ldots \ldots \ldots \ldots \ldots \ldots$

4.3 .1 Measurement Locations . . . . . . . . . . . . . . . . . . 55 
$4.3 .2 \quad$ Data Acquisition System $\ldots \ldots \ldots \ldots$

4.3 .3 Sampling Rates . . . . . . . . . . . . . . . . . . . 58

4.4 Data Reduction Procedures $\ldots \ldots \ldots$. . . . . . . . . . . . . . . . . 59

4.4 .1 Introduction $\ldots \ldots \ldots \ldots \ldots \ldots \ldots \ldots$

$4.4 .2 \quad$ Blade Loading Measurements . . . . . . . . . . . . . . . . . 59

4.4 .3 Three-Hole Probe and Static Probe Data Reduction . . . . . . . . . . . 59

4.4 .4 Rayleigh Shock Correction $\ldots \ldots \ldots \ldots$

4.4.5 Alignment Procedures for Three-Hole and Static Pressure Probe Measurements . . . . . . . . . . . . . . . . . . 63

$4.4 .6 \quad$ Mixing Calculation for Non-Uniform Flow . . . . . . . . . . . . . 65

$4.5 \quad$ Measurement Uncertainty $\ldots \ldots \ldots$

4.5 .1 Introduction $\ldots \ldots \ldots \ldots \ldots \ldots$

4.5 .2 Methodology $\ldots \ldots \ldots \ldots \ldots$

4.5 .3 Uncertainty in Pressure Measurements . . . . . . . . . . . . . . 70

4.5 .4 Uncertainty in Calculated Quantities . . . . . . . . . . . . . 71

$\begin{array}{lll}5 & \text { Computational Procedures } & 73\end{array}$

5.1 Introduction . . . . . . . . . . . . . . . . . . . . . 73

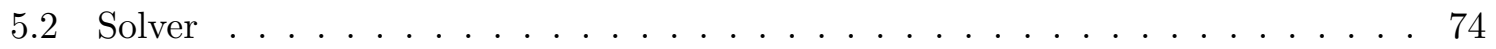

5.3 Mesh . . . . . . . . . . . . . . . . . . . . 76

5.4 Boundary Conditions $\ldots \ldots \ldots \ldots \ldots \ldots \ldots \ldots \ldots$

$6 \quad$ Experimental and Computational Results 82

6.1 Introduction . . . . . . . . . . . . . . . . . . . . . . . 82

$6.2 \quad$ Flow Quality $\ldots \ldots \ldots \ldots \ldots \ldots$

6.2 .1 Introduction . . . . . . . . . . . . . . . . . . 83

6.2 .2 Inlet Pitchwise Flow Uniformity $\ldots \ldots \ldots \ldots$. . . . . . 83

6.2 .3 Outlet Flow Periodicity . . . . . . . . . . . . . . . . . 84

$6.2 .4 \quad$ Axial Velocity Density Ratio (AVDR) $\ldots \ldots \ldots \ldots \ldots$

6.3 Test Cases . . . . . . . . . . . . . . . . . . . . . . . . . 87 
$6.4 \quad$ Blade Loading $\ldots \ldots \ldots \ldots$

6.5 Profile Losses . . . . . . . . . . . . . . . . . . . . . . . . . . . . . . . 94

6.6 Outlet Flow Angle $\ldots \ldots \ldots \ldots$

6.7 Base Pressure . . . . . . . . . . . . . . . . . . . . . . . 98

6.8 Wake Profiles and Blade-to-Blade Flow Features . . . . . . . . . . . . . . 105

6.9 Blade Surface Boundary Layers . . . . . . . . . . . . . . . . . . . . . . . . . . . 128

6.10 Denton (1993) Trailing Edge Loss Prediction . . . . . . . . . . . . . . . 134

6.11 Applicability of the Denton (1993) Trailing Edge Loss Prediction to Compressible Flow . . . . . . . . . . . . . . . . . . . . . 138

\begin{tabular}{|lll}
\hline 7 & Conclusions and Recommendations & 143
\end{tabular}

7.1 Conclusions . . . . . . . . . . . . . . . . . . . . . . . . . . . . 143

7.2 Recommendations . . . . . . . . . . . . . . . . . . . . . 145

\begin{tabular}{ll}
\hline List of References & 146
\end{tabular}

\begin{tabular}{|l|l|}
\hline Appendix A Upstream Reference Total Pressure Calibration & 151
\end{tabular}

Appendix B Incompressible Control Volume Analysis for the Trailing Edge

Losses 154

B.1 Introduction . . . . . . . . . . . . . . . . . . . . . . . . . . . 154

B.2 Continuity Equation . . . . . . . . . . . . . . . . . . 155

B.3 Momentum Equation . . . . . . . . . . . . . . . . . . . . . . . . 157

Appendix C Compressible Control Volume Analysis for the Trailing Edge

Losses 159

C.1 Introduction . . . . . . . . . . . . . . . . . . . . . . . . . . . . . . . 159

C.2 Continuity Equation . . . . . . . . . . . . . . . . . . . 160

C.3 Momentum Equation . . . . . . . . . . . . . . . . . . . . . . . . 161

C.4 Energy Equation . . . . . . . . . . . . . . . . . . . . . . 163

C.5 Solution Method . . . . . . . . . . . . . . . . . . . . . . . . 165 


\section{List of Tables}

2.1 Summary of Studies on Axial Turbine Blades in Steady Compressible Flow . . 9

$3.1 \quad$ Specifications for the Valve Used in the High-Speed Wind Tunnel . . . . . . . . 39

3.2 Typical Cascade Inlet Conditions (Corriveau, 2005:[Jouini[ [2000)] . . . . . . . . 41

$3.3 \quad$ Druck Pressure Transducer Specifications and Roles . . . . . . . . . . . . . . . 42

3.4 Cascade Geometric Parameters . . . . . . . . . . . . . . . . . . . . . . . . . . . 49

4.1 Uncertainty of Measured and Calculated Values (Corriveau, 2005[ Hall[ 2012)] . 72

5.1 Mesh Quality Criteria for the Four Cascades . . . . . . . . . . . . . . . 78

6.1 Summary of Geometry Values Used in the Sieverding et al.(1979) Base Pressure

Correlation . . . . . . . . . . . . . . . . . . . . 102 


\section{List of Figures}

$2.1 \quad$ Flow Features in an Axial Turbine Blade Passage (Corriveau, 2005, Adapted from Jeffries, 2000) $\ldots \ldots \ldots \ldots \ldots \ldots$

2.2 Midspan Loss Components of a Transonic Turbine Cascade, Expressed as Kinetic Energy Loss Coefficient (Mee et al., 1992)] . . . . . . . . . . . . . . . . . 6

$2.3 \quad$ Enthalpy-Entropy Diagram for a Turbine Cascade Flow (Adapted from Denton,

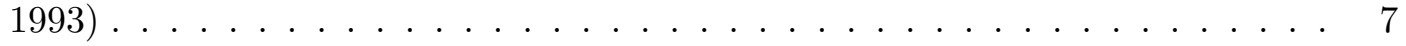

$2.4 \quad$ Schlieren Image of a High Pressure Turbine Cascade at $M_{2}=0.90$ (DetempleLaake $\mid 1989] \ldots \ldots \ldots \ldots \ldots \ldots \ldots$

2.5 Schlieren Image of a High Pressure Turbine Cascade at $M_{2}=1.00$ (DetempleLaake $[1989] \ldots \ldots \ldots \ldots \ldots \ldots$. . . . . . . . . . . . . . . . . . . . . . . . . .

$2.6 \quad$ Schlieren Image of a High Pressure Turbine Cascade at $M_{2}=1.25$ (DetempleLaake $[19890 \ldots \ldots \ldots \ldots \ldots \ldots$

2.7 Shock-Boundary Layer Interaction and Associated Pressure Rise (Graham \& Kost, 1979) . . . . . . . . . . . . . . . . . . . . . . 13

2.8 Profile Loss Data from Corriveau $(2005)$, Showing the Loss "Plateau" Behaviour Roughly Between $M_{2}=1.0$ to $1.2 \ldots \ldots \ldots$. . . . . . . . 13

2.9 Trailing Edge Shock System (Adapted from Denton \& Xu[ 1990) . . . . . . . . 14

2.10 Typical Trailing Edge Pressure Distribution (Adapted from Sieverding et al.,

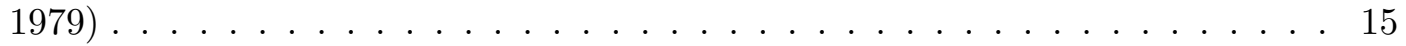

2.11 Behaviour of Base Pressure as a Function of Outlet Static Pressure (Adapted from Sieverding et al. [1979] . . . . . . . . . . . . . . . . . . . 17

2.12 Base Pressure Correlation by Sieverding et al. [1979)] . . . . . . . . . . . . . 18 
2.13 Effect of Reynolds Number on Profile Losses (Hourmouziadis, 1989) . . . . . . 20

2.14 Effect of Turbulence Intensity on the Profile Losses of Three Turbine Cascades: T 104, T 105 and T 106 (Hoheisel et al. [1987)] . . . . . . . . . . . . . . 21

2.15 Effect of AVR on Profile Losses and Base Pressure, Provided for Three Values of Incidence Angle (Rodger et al. $[1992)] \ldots$. . . . . . . . . . . . . . . . . 23

2.16 Profile Loss Coefficients Predicted by the Ainley \& Mathieson[1951) Correlation, Including the Predictions for the Four Cascades Used in this Study . . . . 25

2.17 Correction Factor for Trailing Edge Thickness (t), Non-Dimensionalized by the Blade Pitch (s) (Ainley \& Mathieson, 1951)] . . . . . . . . . . . . . . . 25

2.18 Compressibility Correction Factor (Dunham \& Came, 1970) . . . . . . . . . . . 27

2.19 Trailing Edge Losses from Kacker \& Okapuu [1982), Expressed as Energy Loss Coefficient . . . . . . . . . . . . . . . . . . . 30

2.20 Trailing Edge Total Pressure Loss Coefficient of the Studied Airfoils, as Predicted by $\mid$ Kacker \& Okapuu $[(1982)] \ldots$. . . . . . . . . . . . . . . . . . . . . 31

2.21 Losses Predicted by the Kacker \& Okapuu (1982) Correlation at Midspan for the Cascades Used in the Current Study . . . . . . . . . . . . . . . . . . . 32

2.22 Profile Loss Coefficients Predicted by Seven Profile Loss Correlations (Adapted

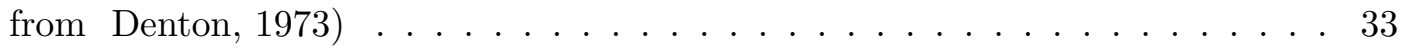

3.1 Pratt \& Whitney Canada High-Speed Wind Tunnel at Carleton University

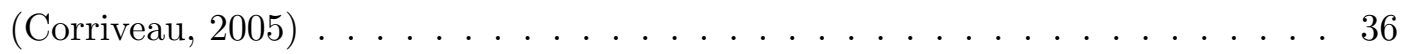

3.2 Broom Wade VC500 Compressor (Corriveau, 2005)] . . . . . . . . . . . . . . 37

3.3 Cascade Test Section (Corriveau, 2005). . . . . . . . . . . . . . . . . . . 39

3.4 Cross-Section of the Inlet Channel and Cascade Assembly (Corriveau[ [2005)] . 40

3.5 Transducer Calibrations Performed by Sooriyakumaran $[(2014)]$. . . . . . . . . 43

3.6 Three-Hole Probe Manufactured by $\operatorname{Islam}[(1999)] \ldots \ldots$. . . . . . . . . . . . . . 44

$3.7 \quad$ Static Probe Manufactured by $\operatorname{Islam}[(1999)] \ldots \ldots$. . . . . . . . . . . . . 45

3.8 Downstream Probe Traversing Mechanism (Adapted from Corriveau, 2005)] . . 46

3.9 Summary of Symbols Used to Define Cascade Geometry (Adapted from Cor-

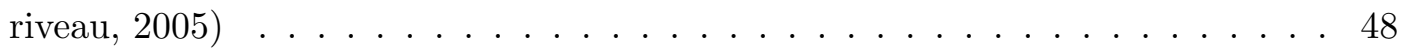


3.10 Profiles of the AF4 and HS1 Family of Cascades (Actual Size) . . . . . . . . . 48

3.11 Surface Curvature of Airfoils, with Airfoil Shape Shown in Background . . . . . 50

4.1 Flow Diagram of the Wind Tunnel Mach Number Control System (Adapted

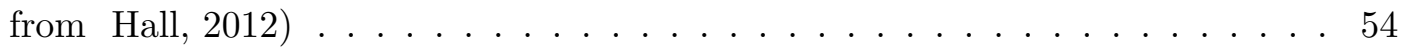

4.2 Measurement Locations in the Cascade Test Section . . . . . . . . . . . 56

$4.3 \quad$ Schematic of the Three-Hole Probe Tip, Showing the Locations of the Three

Pressure Ports . . . . . . . . . . . . . . . . . . 60

4.4 Three-Hole Probe Calibration Curves Used in the Current Study, Collected by Sooriyakumaran $[(2014)] \ldots \ldots \ldots \ldots$. . . . . . . . . . . . . 61

$4.5 \quad$ Flow Quantities around the Probe Tip Shock Wave (Adapted from Sooriyaku-

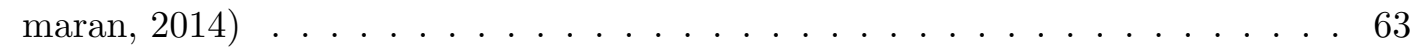

4.6 Static Pressure Pitchwise Misalignment and its Effect on the Local Loss Distribution, at a Mixed-Out Mach Number of 1.25 (Adapted from Sooriyakumaran,

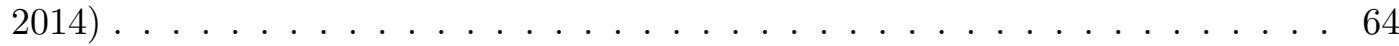

4.7 Control Volume Used for the Mixing Calculation (Adapted from Sooriyaku-

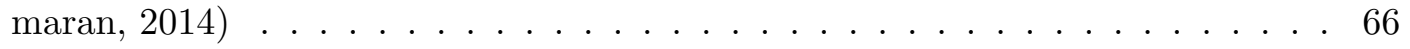

4.8 Variation of Uncertainty on Pressure Measurements (Corriveau, 2005)] . . . . . 71

5.1 Typical Convergence Behaviour of the Simulations . . . . . . . . . . . . 75

5.2 Grid Convergence Study for the AF4 Airfoil . . . . . . . . . . . . . . . . 77

5.3 Mesh Blocking Topology $\ldots \ldots \ldots \ldots$. . . . . . . . . . . . . 79

5.4 Trailing Edge Mesh Detail . . . . . . . . . . . . . . . . . . . 80

$5.5 \quad$ Boundary Conditions Applied to the Fluid Domain . . . . . . . . . . . . . . . 81

$6.1 \quad$ Inlet Mach Number Distribution at $M_{2, i s}=1.3 \ldots \ldots \ldots$. . . . . . 84

6.2 Local Total Pressure Loss Coefficient across the Full Cascade at $M_{2, i s}=1.2$. . 85

6.3 Periodicity of the Mixed-Out Profile Loss Coefficient of the AF4 Cascade at $M_{2, i s}=1.2 \ldots \ldots \ldots \ldots \ldots \ldots$

6.4 Periodicity of the Axial Velocity Density Ratio (AVDR) of the AF4 Cascade

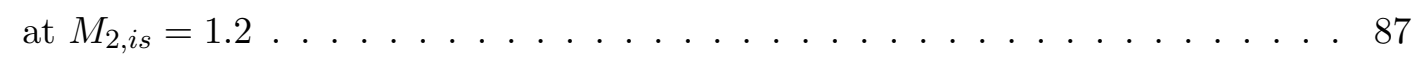


6.5 Mach Number and Reynolds Number Ranges of the Cascades Investigated in the Current Study . . . . . . . . . . . . . . . . . . . 88

6.6 Comparison between Experimental and Numerical Blade Loadings for the AF4 Cascade . . . . . . . . . . . . . . . . . . . . 89

6.7 Blade Loading, Expressed in Terms of the Blade Surface Isentropic Mach Number 92

6.8 Schematic of Trailing Edge Expansion Waves and Shocks, Which Propagate across the Flow Passage $\ldots \ldots \ldots$. . . . . . . . . . . . . . . . . 93

6.9 Effect of Mach Number on the Total Pressure Loss Coefficient. . . . . . . . . . 95

6.10 Effect of Mach Number on the Outlet Flow Angle. . . . . . . . . . . . . . . . . 97

6.11 Base Pressure Distribution around the Trailing Edge Surface of the AF4 cascade as a Function of the Angle of the Blade Surface Normal Vector $\alpha_{\text {surface }}$. . . . 99

6.12 Base Pressure Coefficient $\left(C_{b}\right)$ Variation with Mixed-Out Mach Number . . . . 101

6.13 Definitions of the Symbols Used in the Sieverding et al. (1979) Base Pressure Correlation . . . . . . . . . . . . . . . . . . . . . . . 102

6.14 Comparison of the Base Pressure Measurements of the AF4 Cascade with the

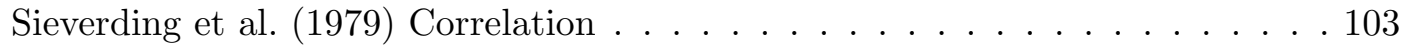

6.15 Comparison of the Base Pressure Measurements of the HS1C Cascade with the Sieverding et al. $(1979)$ Correlation . . . . . . . . . . . . . . . . 103

6.16 Comparison of the Base Pressure Measurements of the HS1A Cascade with the Sieverding et al. $(1979)$ Correlation . . . . . . . . . . . . . . . . . . . 104

6.17 Comparison of the Base Pressure Measurements of the HS1D Cascade with the Sieverding et al. $(1979)$ Correlation . . . . . . . . . . . . . . . . . . . . . 104

6.18 Blade-to-Blade Flow Features and Wake Profile of the AF4 Cascade at a MixedOut Mach Number of 0.77 (Profile Losses and Base Pressure Inset) . . . . . . . 107 6.19 Blade-to-Blade Flow Features and Wake Profile of the AF4 Cascade at a MixedOut Mach Number of 0.96 (Profile Losses and Base Pressure Inset) . . . . . . . 108

6.20 Blade-to-Blade Flow Features and Wake Profile of the AF4 Cascade at a MixedOut Mach Number of 1.16 (Profile Losses and Base Pressure Inset) . . . . . . . 109 
6.21 Blade-to-Blade Flow Features and Wake Profile of the AF4 Cascade at a MixedOut Mach Number of 1.34 (Profile Losses and Base Pressure Inset)] . . . . . . 110

6.22 Blade-to-Blade Flow Features and Wake Profile of the AF4 Cascade at a MixedOut Mach Number of 1.37 (Profile Losses and Base Pressure Inset)] . . . . . . 111

6.23 Blade-to-Blade Flow Features and Wake Profile of the AF4 Cascade at a MixedOut Mach Number of 1.38 (Profile Losses and Base Pressure Inset) . . . . . . 112

6.24 Blade-to-Blade Flow Features and Wake Profile of the HS1A Cascade at a

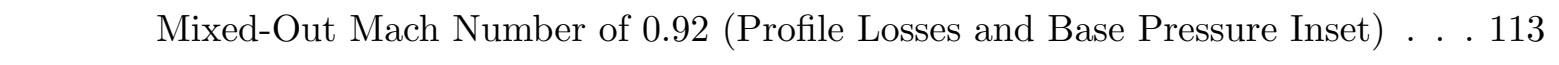

6.25 Blade-to-Blade Flow Features and Wake Profile of the HS1A Cascade at a Mixed-Out Mach Number of 1.01 (Profile Losses and Base Pressure Inset) . . . 114

6.26 Blade-to-Blade Flow Features and Wake Profile of the HS1A Cascade at a Mixed-Out Mach Number of 1.24 (Profile Losses and Base Pressure Inset) . . . 115

6.27 Blade-to-Blade Flow Features and Wake Profile of the HS1A Cascade at a Mixed-Out Mach Number of 1.29 (Profile Losses and Base Pressure Inset) . . . 116

6.28 Blade-to-Blade Flow Features and Wake Profile of the HS1A Cascade at a Mixed-Out Mach Number of 1.32 (Profile Losses and Base Pressure Inset)] . . . 117 6.29 Blade-to-Blade Flow Features and Wake Profile of the HS1C Cascade at a

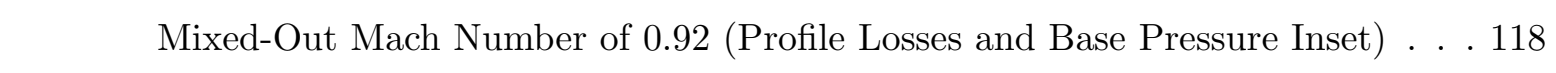

6.30 Blade-to-Blade Flow Features and Wake Profile of the HS1C Cascade at a Mixed-Out Mach Number of 1.06 (Profile Losses and Base Pressure Inset) . . . 119

6.31 Blade-to-Blade Flow Features and Wake Profile of the HS1C Cascade at a Mixed-Out Mach Number of 1.23 (Profile Losses and Base Pressure Inset) . . . 120

6.32 Blade-to-Blade Flow Features and Wake Profile of the HS1C Cascade at a Mixed-Out Mach Number of 1.27 (Profile Losses and Base Pressure Inset) . . . 121

6.33 Blade-to-Blade Flow Features and Wake Profile of the HS1D Cascade at a Mixed-Out Mach Number of 0.92 (Profile Losses and Base Pressure Inset) . . . 122

6.34 Blade-to-Blade Flow Features and Wake Profile of the HS1D Cascade at a Mixed-Out Mach Number of 1.01 (Profile Losses and Base Pressure Inset)] . . . 123 
6.35 Blade-to-Blade Flow Features and Wake Profile of the HS1D Cascade at a Mixed-Out Mach Number of 1.23 (Profile Losses and Base Pressure Inset) . . . 124

6.36 Blade-to-Blade Flow Features and Wake Profile of the HS1D Cascade at a Mixed-Out Mach Number of 1.30 (Profile Losses and Base Pressure Inset) . . . 125

6.37 Effect of Mach Number on the Boundary Layer Displacement Thickness $\left(\delta^{*}\right)$ and Momentum Thickness $(\theta)$ at $95 \%$ Axial Chord $\ldots . . . . . . .130$

6.38 Effect of the Outlet Mach Number on the Shape Factor of the Suction Surface

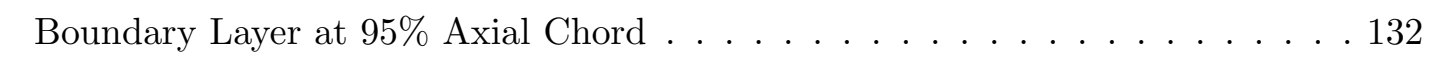

6.39 Trailing Edge Loss Prediction from Denton $[1993)]$. . . . . . . . . . . . . . . 136

6.40 Trailing Edge Loss Prediction from Denton $[1993)$ Compared to the Mixed-Out Profile Losses, Both Using Data from the Simulations . . . . . . . . . . . . . 139

6.41 Effect of Mach Number on the Compressible Trailing Edge Loss Prediction of the AF4 Cascade, Using Constant Values of $C_{b}, \delta^{*}$ and $\theta$. . . . . . . . . . 140

6.42 Effect of Individually Varying Each Parameter on the Trailing Edge Loss Prediction for Compressible Flow . . . . . . . . . . . . . . . . . . . . . 141

A.1 $\quad$ Pitot tube used for upstream total pressure measurements . . . . . . . . . . . 152

A.2 4th order polynomial curve fit of the correction factor for inlet total pressure . 153

B.1 Control Volume Defined Downstream of the Trailing Edge of a Row of Staggered Blades in Incompressible Flow (Adapted from Denton, [1993)] . . . . . . . . . 154

C.1 Control Volume Defined Downstream of the Trailing Edge of a Row of Staggered Blades in Compressible Flow (Adapted from Denton[] 1993)] . . . . . . . . . . . 159 


\section{List of Symbols}

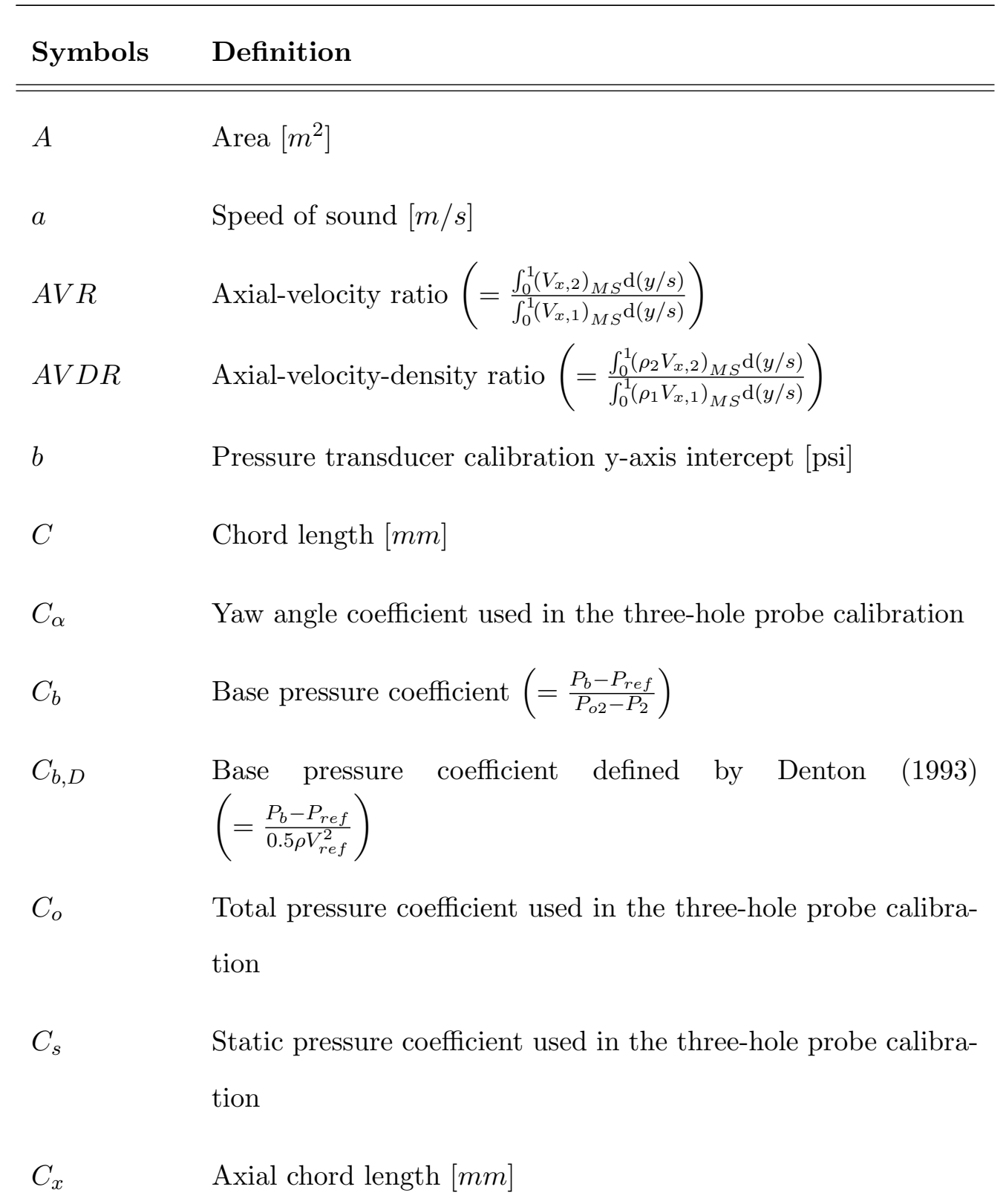




\begin{tabular}{|c|c|}
\hline$C F M$ & Compressibility correction used in Kacker \& Okapuu (1982) \\
\hline$H$ & Shape factor $\left(=\frac{\delta^{*}}{\theta}\right)$ \\
\hline$h$ & Blade span $[\mathrm{mm}]$ \\
\hline$i_{\text {des }}$ & Design incidence $\left[^{\circ}\right]$ \\
\hline$k_{C}$ & Compressibility correction used in Dunham \& Came (1970) \\
\hline$K_{P}$ & Mach number loss correction factor \\
\hline$M$ & $\operatorname{Mach}$ number $\left(=\frac{u}{a}=\sqrt{\left.\frac{2}{(\gamma-1)}\left(\left(\frac{P_{o}}{P}\right)^{\frac{\gamma-1}{\gamma}}-1\right)\right)}\right.$ \\
\hline$M_{i s}$ & Isentropic Mach number $\left(=\sqrt{\frac{2}{(\gamma-1)}}\left(\left(\frac{P_{o 1}}{P}\right)^{\frac{\gamma-1}{\gamma}}-1\right)\right.$ \\
\hline$\left(M_{s}\right)_{x}$ & Blade surface isentropic Mach number \\
\hline$m$ & Pressure transducer calibration slope $[p s i / V]$ \\
\hline$\dot{m}$ & Mass flow rate $[\mathrm{kg} / \mathrm{s}]$ \\
\hline$o$ & Throat opening $[\mathrm{mm}]$ \\
\hline$P$ & Pressure $[P a]$ \\
\hline$P_{b}$ & Base pressure $[P a]$ \\
\hline$\left(P_{s}\right)_{x}$ & Blade surface static pressure $[\mathrm{Pa}]$ \\
\hline$q$ & Dynamic pressure $\left(=0.5 \rho V^{2}\right)$ \\
\hline \multirow[t]{2}{*}{$R$} & Local surface radius of curvature $[\mathrm{mm}]$ or ideal gas constant for \\
\hline & $\operatorname{air}(=287.058 J / k g K)$ \\
\hline$R e$ & Reynolds Number $\left(=\frac{\rho_{2} V_{2} C}{\mu}\right)$ \\
\hline
\end{tabular}


$R_{\theta t} \quad$ Boundary layer momentum thickness Reynolds number for transition $\left(=\frac{\rho_{e} V_{e} \theta}{\mu}\right)$

$s$

$T$

$t$

$T u$

$u$

V

$v$

$W_{e}$

$w$

$x$

Y

$y$

$y^{+}$

Z

$z$

\section{Greek}

$\alpha$
Blade pitch $[\mathrm{mm}]$

Temperature $\left[K\right.$ or $\left.{ }^{\circ} C\right]$

Trailing edge thickness $[\mathrm{mm}]$

Turbulence intensity

Component of velocity in the x-direction $[\mathrm{m} / \mathrm{s}]$

Velocity $[\mathrm{m} / \mathrm{s}]$ or measured voltage $[\mathrm{V}]$

Component of velocity in the y-direction $[\mathrm{m} / \mathrm{s}]$

Trailing edge wedge angle $\left[^{\circ}\right]$

Component of velocity in the z-direction $[\mathrm{m} / \mathrm{s}]$ or dimension $(=t+o)[m m]$

$\mathrm{X}$ coordinate $[m]$

Total pressure loss coefficient $\left(=\frac{P_{o 1}-P_{o 2}}{P_{o 2}-P_{2}}\right)$

Y coordinate $[m]$

Non-dimensional distance from the wall $\left(=\frac{\rho_{w} \sqrt{\frac{\tau_{w}}{\rho_{w}}} y}{\mu_{w}}\right)$

Zweifel Coefficient

$\mathrm{Z}$ coordinate $[m]$ 


$\begin{array}{ll}\beta_{2}^{*} & \text { Gauging angle }\left[^{\circ}\right] \\ \delta & \left.\text { Flow deviation angle from the outlet metal angle }{ }^{\circ}\right] \\ \delta^{*} & \text { Boundary layer displacement thickness } \quad[\mathrm{mm}] \\ & \left(=\int_{0}^{y_{e}}\left(1-\frac{\rho(y) V(y)}{\rho_{e} V}\right) \mathrm{d} y\right) \\ \eta & \text { Overall turbine efficiency } \\ \gamma & \text { Ratio of specific heats }(1.4 \text { for air }) \\ \mu & \text { Dynamic viscosity }\left[N s / \mathrm{m}^{2}\right] \\ \nu & \text { Kinematic viscosity }\left[\mathrm{m}^{2} / \mathrm{s}\right] \\ & \end{array}$

$\Delta \phi^{2} \quad$ Kinetic energy loss coefficient

$\rho \quad$ Density $\left[\mathrm{kg} / \mathrm{m}^{3}\right]$

$\tau \quad$ Shear stress $[P a]$

$\theta$ Boundary layer momentum thickness $[\mathrm{mm}]$

$\left(=\int_{0}^{y_{e}} \frac{\rho(y) V(y)}{\rho_{e} V_{e}}\left(1-\frac{V(y)}{V_{e}}\right) \mathrm{d} y\right)$

$\theta_{u} \quad$ Unguided turning angle $\left[^{\circ}\right]$

$\zeta \quad$ Energy or enthalpy loss coefficient, or blade stagger angle $\left[^{\circ}\right]$

Subscript

1

Upstream conditions

2

Downstream mixed-out conditions (unless indicated otherwise)

atm Atmospheric conditions 
e

is

$M S$

$\max$

mix

$O$

$P$

ref

S

SHOCK

TET or TE

$T C$

$w$

\section{Acronyms}

AMDC

Ainley and Methieson and Dunham and Came

CFD

HPT

HSWT

$\mathrm{KO}$

Computational fluid dynamics

High pressure turbine

High speed wind tunnel

Kacker and Okapuu
Conditions at the edge of the boundary layer

Conditions reached through an isentropic process

Midspan

Maximum (used for maximum thickness $t_{\max }$ )

Mixed-out conditions, far downstream of cascade

Stagnation conditions

Profile losses or static conditions behind the probe tip shock, as observed by the three-hole probe

Reference conditions $\left(P_{o, r e f}\right.$ : conditions measured far upstream of the cascade)

Secondary losses

Shock losses

Trailing edge losses

Tip clearance losses

Value at the wall or blade surface 
LE Leading edge

LPT Low pressure turbine

PS Pressure side or pressure surface

SS Suction side or suction surface

TE Trailing edge

TRL Technological readiness level 


\section{Chapter 1}

\section{Introduction}

While gas turbine technology continues to mature, the global technological needs for transportation and energy are shifting increasingly towards higher fuel efficiency, low emissions and low noise operation. As such, current gas turbine designs are well optimized to balance performance, efficiency and economics of manufacturing and operation. Improving upon these well balanced machines requires a good understanding of the flow mechanisms within the turbomachinery.

During the preliminary design of axial turbine blades, it is necessary to estimate the losses around the blades in order to predict the efficiency of the turbine, and thus determine whether the turbine meets the design requirements. In the early stages of design, the losses are typically estimated using empirical correlations based on a small number of aerodynamic and geometric parameters. In order to accurately reflect improvements in the state of the art, loss correlations must be updated periodically.

An area of possible improvement in turbine loss correlations is the prediction of losses at transonic and supersonic Mach numbers. It is desirable to operate turbine blades at these high Mach numbers because, for the same aerodynamic blade loading, higher turbine rotor speeds allow more work to be extracted from a turbine stage. Increasing the rotor speed can thus allow the number of stages to be reduced, leading to a lighter design. For this reason, transonic Mach numbers are common in aeroengines, despite the penalty in losses that transonic blades suffer over subsonic ones. However, the profile losses of turbine blades 
in transonic flows are still not adequately captured by the current loss correlations, which hampers the preliminary design of an efficient transonic turbine.

Experimental research in gas turbine flow physics is typically done in either a linear cascade of turbine blades or a cold flow rotating test rig. While the rotating test rig more closely approximates the flow observed in an operational turbine, the rotating equipment imposes limitations on instrumentation. The linear cascade, though lacking rotational effects, can offer more insight into the fundamental features that arise in flow around blade profiles. Linear cascades are therefore used to evaluate concepts at technology readiness levels (TRL) of about 1 to 3 . Carleton University is home to one such linear cascade facility, namely the Pratt \& Whitney Canada High-Speed Wind Tunnel (HSWT), which is an intermittent blow-down wind tunnel.

The objective of the current investigation is to clarify the flow mechanisms that drive the profile losses in the transonic and supersonic regimes. This was accomplished through wind tunnel measurements in the high-speed wind tunnel, and also through numerical simulations. The behaviour of four linear cascades of high-pressure turbine blades was investigated. Aerodynamic data were collected at a fine resolution of Mach numbers in order to clearly identify the effect of Mach number on the profile losses. The effect of the base pressure (the pressure in the region of separated flow just downstream of the trailing edge) was also investigated, as well as the character of the boundary layer along the blade surface. A quantitative estimate of the trailing edge losses was then made in order to situate the relative contribution of trailing edge losses to the profile losses.

The next chapter will provide an overview of the current state of knowledge regarding profile losses in an axial turbine. The main factors contributing to the profile losses will be explained, and the current methods of profile loss prediction will be presented. Chapter 3 will describe the experimental apparatus, including the high-speed wind tunnel and the four cascades under investigation. The experimental procedures will be provided in Chapter 4, and the numerical procedures used to simulate the cascade flows will be provided in Chapter 5 . All experimental and numerical results and discussion will be presented in Chapter 6, followed by the conclusions in Chapter 7 . 


\section{Chapter 2}

\section{Literature Review}

\subsection{Introduction}

The current study examines the effects of compressibility on the profile losses in axial turbines at transonic and supersonic conditions. The present chapter begins by providing a summary of the main flow features of transonic axial turbines. Based on these flow features, the overall losses can be subdivided into loss components, including profile losses. The parameters that affect profile losses, such as Mach number and the Reynolds number, will then be examined. Finally, a description of existing loss correlations will be presented, and it will be shown where these loss correlations could be improved.

\subsection{Flow Features Within an Axial Turbine Blade Row}

The large pressure and velocity gradients present in axial turbine flows introduce a number of characteristic flow features. Given the complexity of these flow features, their study is still an area of active research.

The main flow features of an axial turbine blade row are shown schematically in Figure 2.1 . The blades are bounded by two endwalls. The hubwall and tipwall are the endwalls corresponding to the blade hub and blade tip,respectively. Due to the presence of a stagnation point at the airfoil leading edge, an adverse pressure gradient occurs upstream of the airfoil. The adverse pressure gradient causes separation to occur in the endwall boundary layer, and 


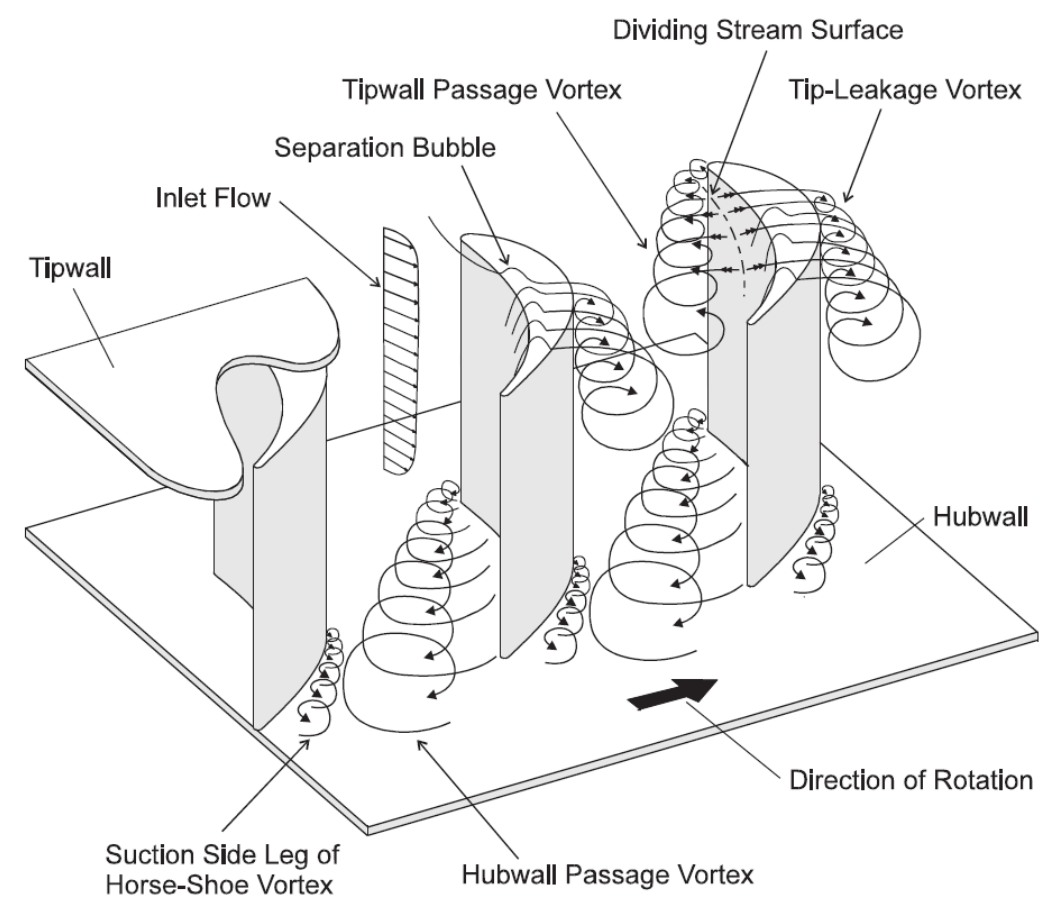

Figure 2.1: Flow Features in an Axial Turbine Blade Passage (Corriveau, 2005, Adapted from Jeffries, 2000)

the separated fluid curls up into a vortex at the blade leading edge. This "horseshoe" vortex is generated and swept back on either side of the blade, creating the suction side and pressure side legs of the horseshoe vortex.

Due to the pressure gradient between the blade suction surface and pressure surface, the pressure side leg of the horseshoe vortex is swept across the blade passage, along with the low momentum endwall boundary layer fluid inside the passage. This boundary layer fluid is added to the fluid in the pressure side leg of the horseshoe vortex, increasing its size. The combined structure becomes what is called the passage or secondary vortex.

In rotating blade rows, a gap exists between the rotating blade tips and the stationary endwall (or tipwall). This small tip clearance allows fluid to flow from the pressure side to the suction side of the blade, causing a jet of fluid to be ejected next to the suction side leg of the horseshoe vortex. The ejected fluid's interaction with the main passage flow causes a strong tip leakage vortex to form. Since the linear cascade used in the current study has no 
tip clearance, the flow at both ends of the blade resembles the flow at the hubwall shown in Figure 2.1.

\subsection{Loss Components Within an Axial Turbine Blade Row}

Flow features that cause viscous mixing have the effect of reducing the efficiency of the turbine. These flow features are called losses since they reduce the turbine's power output. The "lost" power results from thermodynamic irreversibilities and is irrecoverable. As described in Denton (1993), the overall losses in the turbine are historically estimated by dividing the losses into components. The components are typically evaluated independently and then summed together. In reality, it is recognized that the components are seldom truly independent. However, the method of treating components separately is still used to provide useful loss estimates. The typical loss components are the following:

- Profile Losses

- Secondary Losses

- Tip-Leakage Losses

Profile losses are the losses generated by the flow around the two-dimensional airfoil cross-section, unaffected by the flow features near the endwalls. Profile losses arise in the blade surface boundary layers, in the shocks that form when there are regions of supersonic flow, and in the separated flow behind the trailing edge. Figure 2.2 shows the relative magnitudes of these loss components at different Mach numbers for a particular transonic turbine cascade.

Secondary losses sometimes called endwall losses, result from the viscous mixing of the secondary vortices described in Section 2.2 .

Tip leakage losses occur due to the leakage flow around rotating blades. These losses originate from the viscous mixing in the narrow tip gap, in the tip-leakage vortex and in the vortex's interactions with the surrounding flow, following a mixing process similar to the secondary losses. 


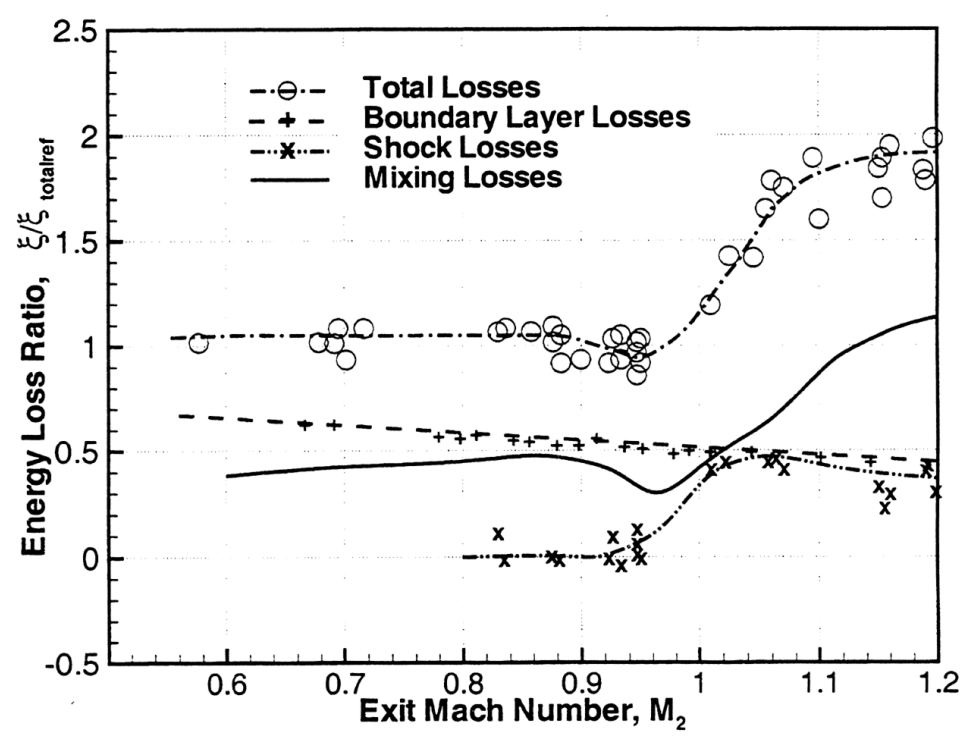

Figure 2.2: Midspan Loss Components of a Transonic Turbine Cascade, Expressed as Kinetic Energy Loss Coefficient (Mee et al., 1992)

The current study examines the midspan flow of a two-dimensional cascade. Since this flow is approximately two-dimensional and well away from endwall flow features, the midspan losses are considered representative of profile losses.

\subsection{Loss Coefficient Definitions}

From a thermodynamic standpoint, losses cause a reduction in performance as a result of an increase in entropy. Although a rise in entropy is the most fundamental definition of loss, this definition is cumbersome to use as a loss coefficient since entropy cannot be measured directly. It is more convenient to express losses in terms of measurable quantities, such as those presented in the entropy-enthalpy diagram in Figure 2.3.

The total pressure loss coefficient $(Y)$ is used in many loss prediction models such as Ainley \& Mathieson (1951) and Kacker \& Okapuu (1982), and will be the primary method of expressing losses in this thesis. For a turbine, $Y$ is defined as:

$$
Y=\frac{P_{o 1}-P_{o 2}}{P_{o 2}-P_{2}}
$$




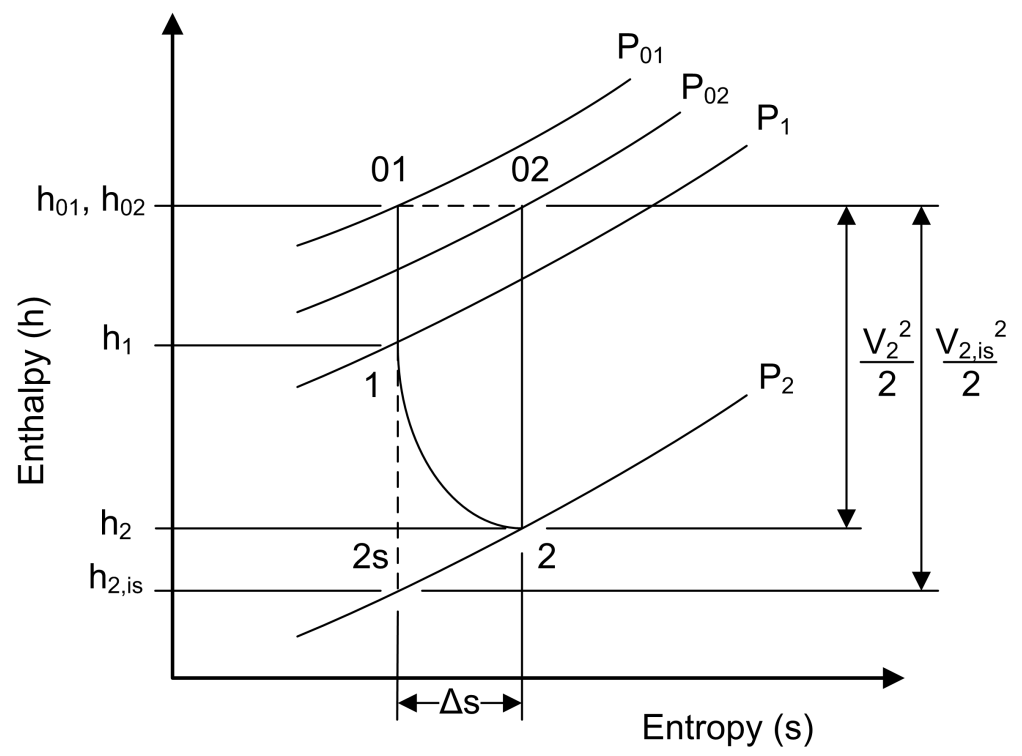

Figure 2.3: Enthalpy-Entropy Diagram for a Turbine Cascade Flow (Adapted from Denton, 1993)

where $P_{o 1}$ is the mass-averaged total pressure at the inlet and $P_{o 2}$ and $P_{2}$ are the mixed-out total and static pressures at the outlet, respectively.

The energy or enthalpy loss coefficient $(\zeta)$ is defined by Denton (1993) as:

$$
\zeta=\frac{h_{2}-h_{2, i s}}{h_{o 2}-h_{2}}
$$

where the outlet isentropic enthalpy $\left(h_{2, i s}\right)$ is the enthalpy that would be obtained from an isentropic expansion to the same outlet static pressure as the real process. The numerator $\left(h_{2}-h_{2, i s}\right)$ is therefore the static enthalpy rise caused by the increase in entropy.

Kacker \& Okapuu (1982) and Mee et al. (1992) use a slightly different definition of energy loss coefficient $\left(\Delta \phi^{2}\right)$.

$$
\Delta \phi^{2}=\frac{h_{2}-h_{2, i s}}{h_{o 2}-h_{2, i s}}
$$

The total enthalpy $\left(h_{0}\right)$ can be expressed in terms of the static enthalpy $(h)$ and the flow velocity $(V)$.

$$
h_{0}=h+\frac{V^{2}}{2}
$$


This relationship is shown graphically in Figure 2.3, where the difference between the total and static enthalpy is given by the velocity term. Equation 2.3 can thus be rearranged into the following form.

$$
\Delta \phi^{2}=1-\frac{V_{2}^{2}}{V_{2, i s}^{2}}
$$

For this reason, $\Delta \phi^{2}$ is often called the kinetic energy loss coefficient.

\subsection{Aerodynamic Influences on Midspan Losses}

\subsubsection{Mach Number}

Higher rotor speeds allow more work to be extracted from a stage for the same aerodynamic blade loading. Thus, higher rotor speeds can lead to lighter designs if the number of stages can be reduced. For this reason, transonic Mach numbers in turbines are common in aeroengines, despite the penalty in losses that transonic blades suffer over subsonic ones.

Many studies of high speed cascades are concerned with the effect of Mach number on losses. Examples of relevant research are summarized in Table 2.1. However, in cascade testing it can be difficult to vary the Mach number without also varying the Reynolds number, since both parameters are functions of the flow velocity. Some studies attempt to separate the two flow parameters by varying the density independently of the pressure ratio across the cascade. Mee et al. (1992) and Hall (2012) find that the Mach number does not greatly affect the profile losses in the subsonic regime. Though the Mach number has a pronounced effect on the blade loading, the resulting effect on the boundary layer loss is moderate. However, in the transonic regime, it is found that the appearance of shock waves and pockets of supersonic flow causes significant increases in loss.

Typical shock wave patterns can be observed in the schlieren images presented by Detemple-Laake (1989), as shown in Figures 2.4 to 2.6. The appearance of shocks begins at a critical outlet Mach number below $M_{2}=1.0$. Local regions of supersonic flow form along the blade suction surface, visible as weak expansion fans and ending in normal shocks, as shown in Figure 2.4. As the Mach number is increased, trailing edge shocks form, visible 
Table 2.1: Summary of Studies on Axial Turbine Blades in Steady Compressible Flow

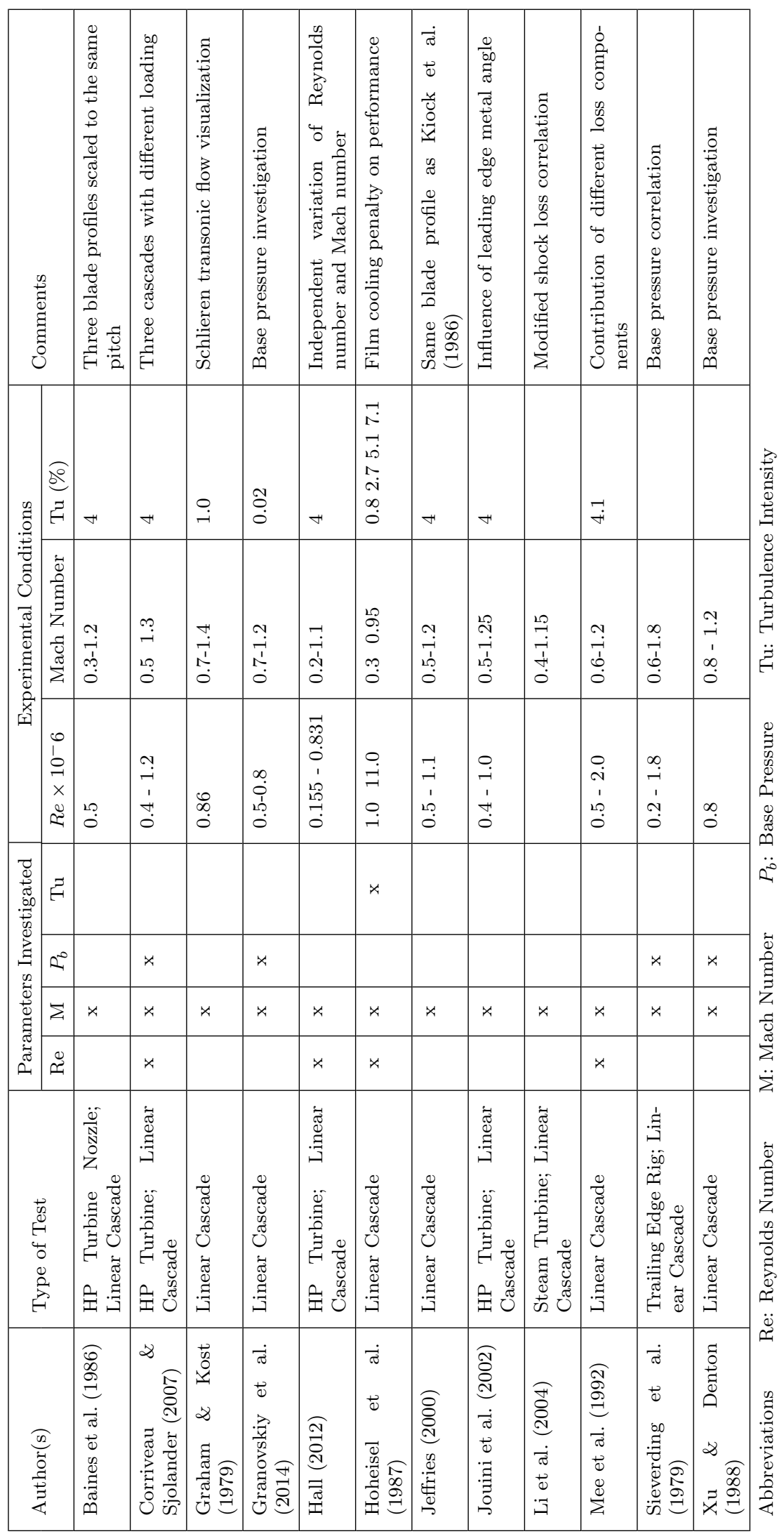




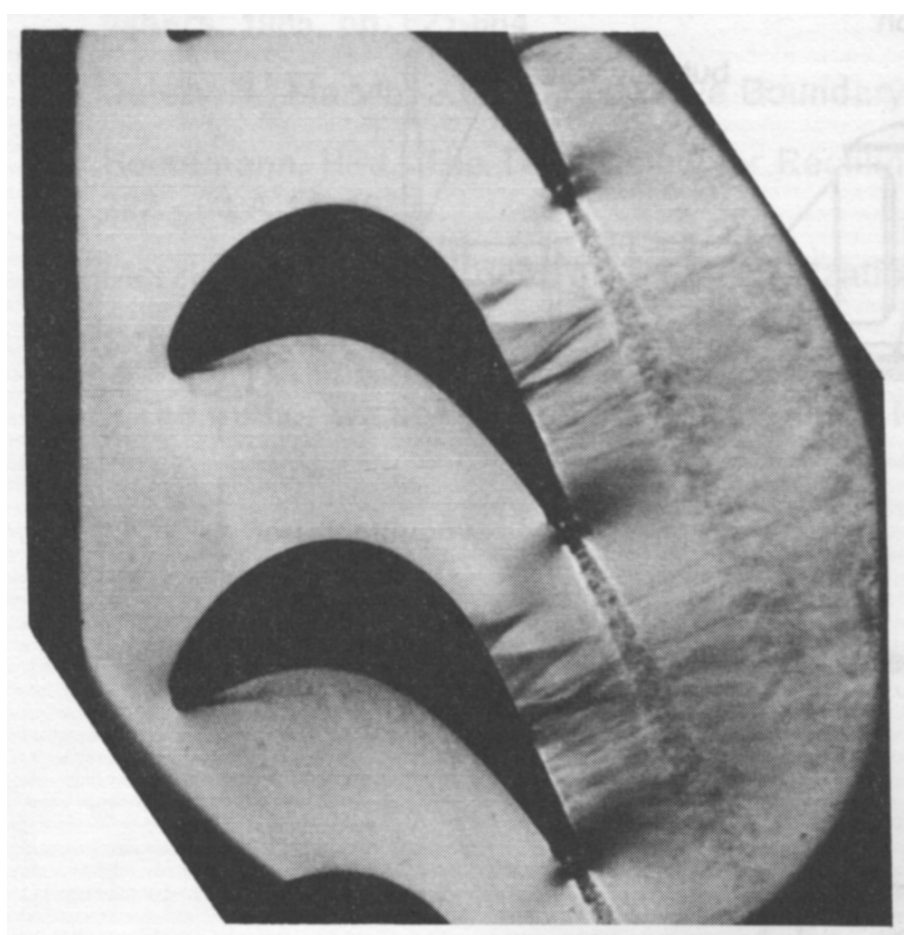

Figure 2.4: Schlieren Image of a High Pressure Turbine Cascade at $M_{2}=0.90$ (DetempleLaake, 1989)

in Figure 2.5. Further increasing the Mach number causes the trailing edge shock system to strengthen and extend further into the flow passage, eventually reaching the suction surface of the adjacent blade. Sufficiently strong shocks are reflected off this surface, as shown in Figure 2.6. The trailing edge shocks become more oblique and tend to sweep back along the adjacent blade's suction surface. At a high enough Mach number, the trailing edge shocks become so oblique that they sweep clear of the trailing edges of the adjacent blade.

The shocks described above generate losses in two ways. The most obvious source of losses is the entropy generated within the shock. However, an additional source of losses comes from the interaction of shock waves with the boundary layers on the adjacent blade surfaces. Shocks cause the static pressure to rise in the streamwise direction, thus imposing a strong local adverse pressure gradient on the boundary layer. A boundary layer separation bubble almost certainly forms, and complete boundary layer separation often occurs. In both cases, viscous dissipation inside and downstream of the shock-boundary layer interaction is a source of losses (Denton, 1993). 


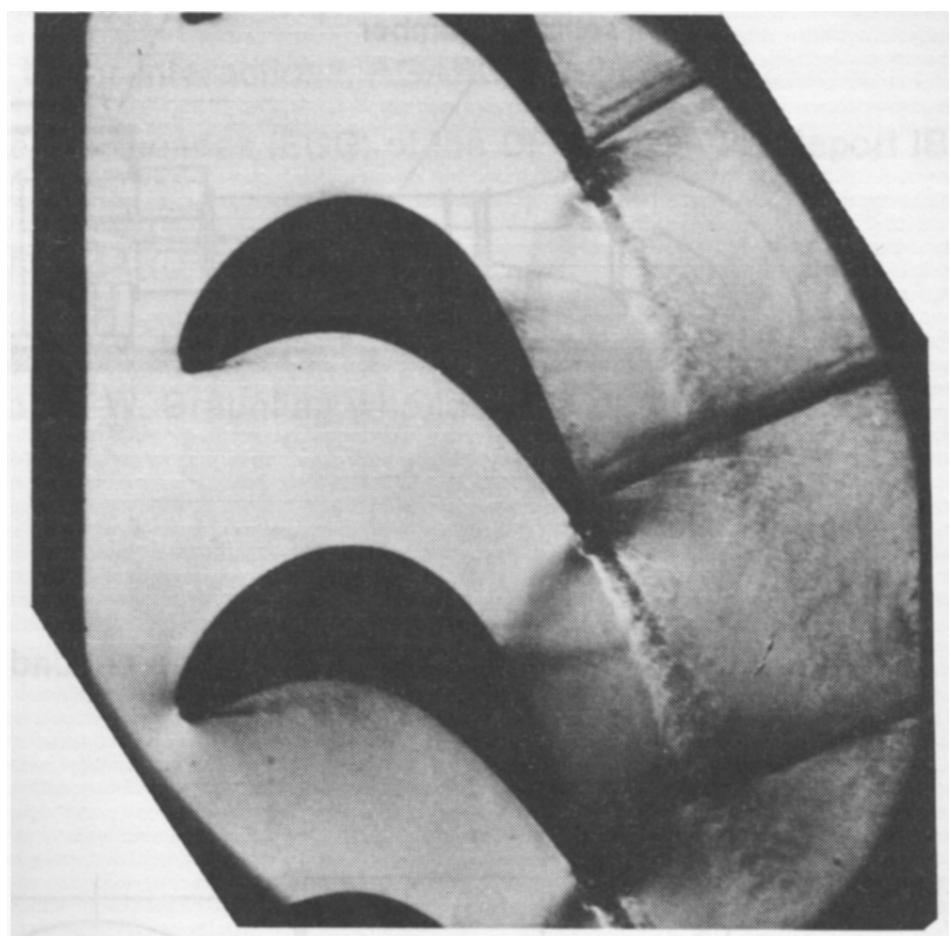

Figure 2.5: Schlieren Image of a High Pressure Turbine Cascade at $M_{2}=1.00$ (DetempleLaake, 1989)

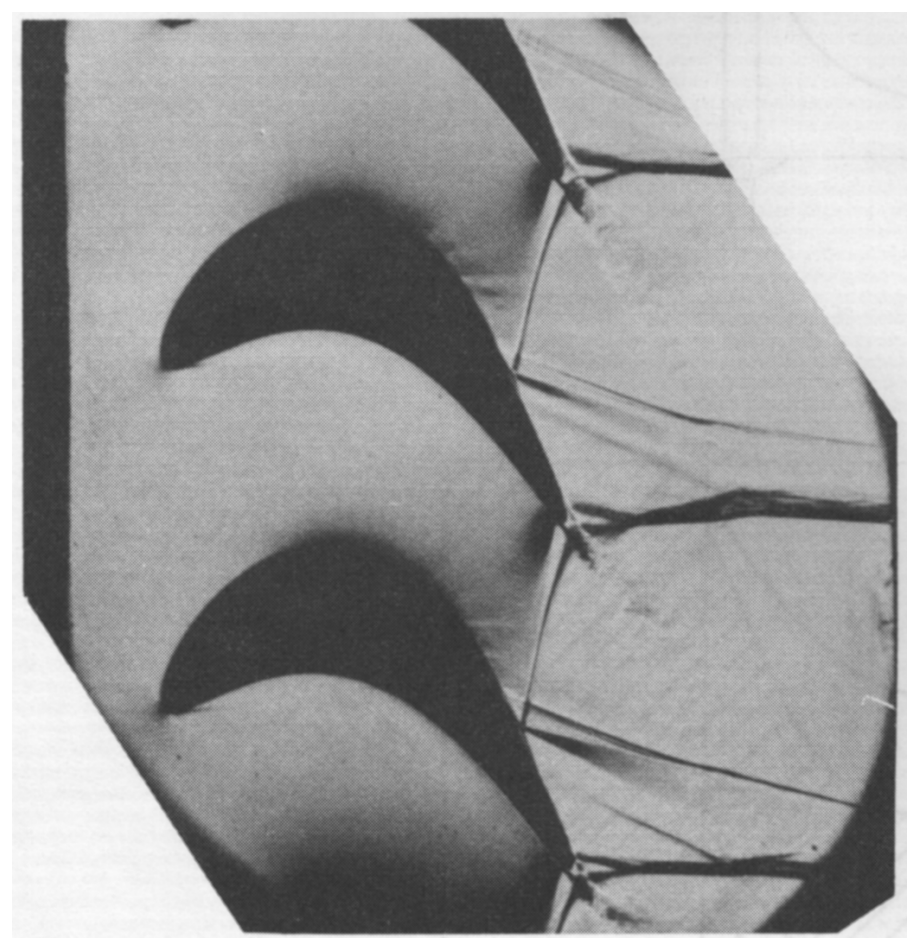

Figure 2.6: Schlieren Image of a High Pressure Turbine Cascade at $M_{2}=1.25$ (DetempleLaake, 1989) 
The mechanisms of the shock-boundary layer interaction are described by Graham \& Kost (1979), and in more detail by Delery \& Marvin (1986). The main features are shown in Figure 2.7. Shock waves, in order to obey the second law of thermodynamics, must compress the fluid and cause a pressure rise. This compression occurs over an infinitesimally small distance in the shock wave. However, in the boundary layer, where there is subsonic flow, the pressure rise is distributed over a larger streamwise distance. Unlike in supersonic flow, the pressure rise is transmitted upstream in the subsonic flow, causing the boundary layer to thicken and possibly separate. At the point of separation, compression waves are generated that coalesce into a shock away from the surface. The separated boundary layer thus becomes a shear layer which moves away from the surface, displacing the adjacent freestream flow. The increased shear forces and entrainment of the freestream fluid tend to force the shear layer back down, and the flow turning generates an expansion fan. The boundary layer typically reattaches downstream of the shock. However, the flow may remain fully separated with a sufficiently strong shock or high surface curvature. If the boundary layer reattaches, a second compression shock is generated. In that case, the two compression shocks propagate downstream and appear to be "reflected" from the original incoming shock.

As a result of the complex interactions between the shock patterns and the boundary layers, particularly around the trailing edge, the effect of Mach number on the profile losses is equally complex. A typical profile loss curve is shown in Figure 2.8, from Corriveau (2005). The curve shows a significant increase in profile losses just before Mach 1.0 resulting from the formation of shocks. After this increase, the profile loss coefficient typically levels off and sometimes even decreases, before resuming its sharp increase at higher supersonic Mach numbers. This loss "plateau" has been observed by many studies, including Sieverding (1985), Chen (1987), Xu \& Denton (1988) Mee et al. (1992), and, more recently, Li et al. (2004), Corriveau (2005) and Granovskiy et al. (2014).

The shape of the profile loss curve is highly dependent on the blade geometry. It is also suggested by Sieverding et al. (1979) that the supersonic profile loss behaviour is related to the base pressure, or the static pressure aft of the trailing edge, since the base pressure is strongly linked to the trailing edge losses. However, the exact mechanisms leading to the loss 


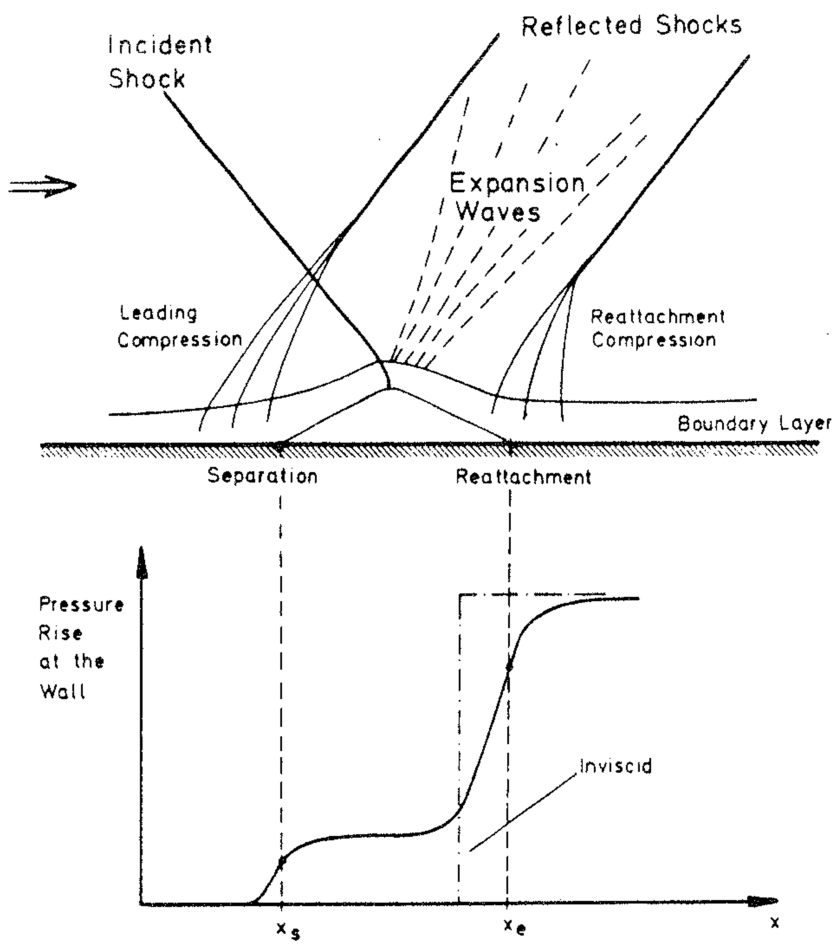

Figure 2.7: Shock-Boundary Layer Interaction and Associated Pressure Rise (Graham \& Kost, 1979)

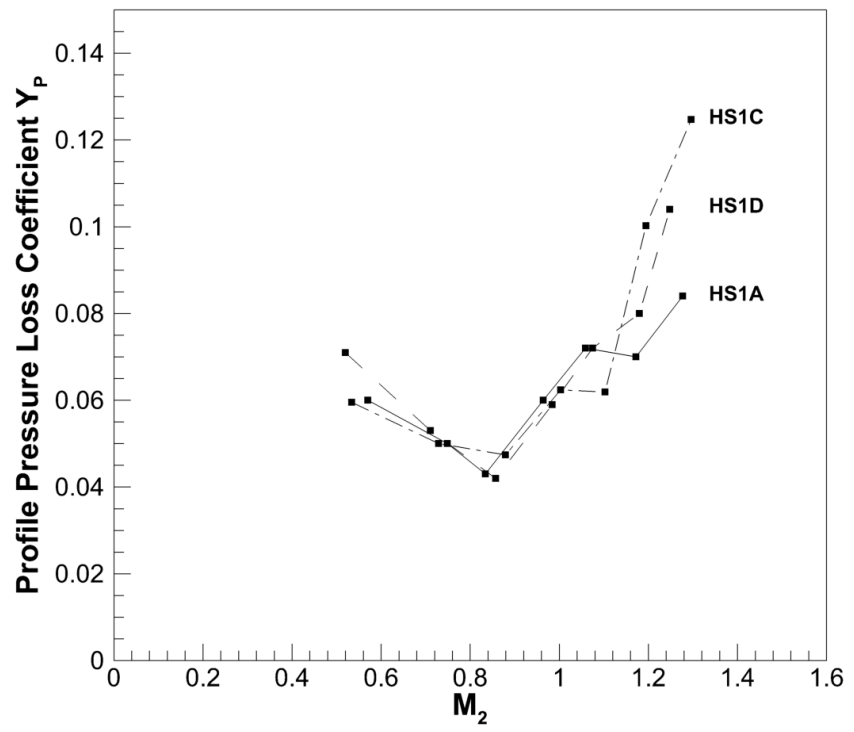

Figure 2.8: Profile Loss Data from Corriveau (2005), Showing the Loss "Plateau" Behaviour Roughly Between $M_{2}=1.0$ to 1.2 


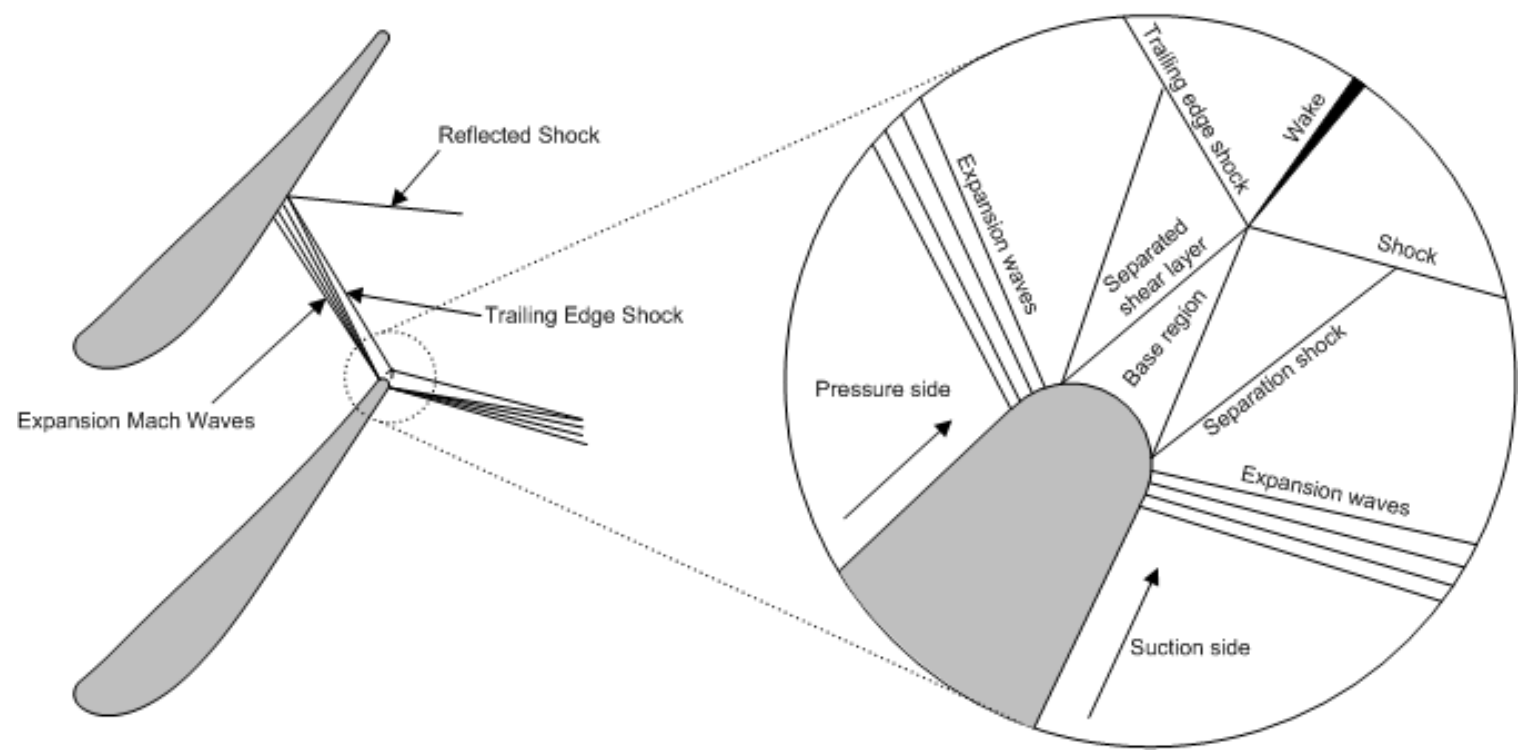

Figure 2.9: Trailing Edge Shock System (Adapted from Denton \& Xu, 1990)

plateau are not well understood. It is one of the objectives of the current study to investigate these mechanisms further.

\subsubsection{Base Pressure}

Base pressure refers to the static pressure within the region of separated flow directly behind the trailing edge of the blade. In the supersonic flow regime, a complex system of expansion fans and shock waves forms at the trailing edge, as shown in Figure 2.9.

When supersonic flow first begins to curve around the trailing edge, an expansion fan is created as the flow expands to remain attached to the surface. Shortly after the expansion fan, the flow separates, forming a compression corner and causing a weak separation shock. A shear layer is formed between the high speed flow and the separated fluid in the base region. When the suction and pressure side flows converge, the compression corner required to align the two flows generates the trailing edge shock.

In Figure 2.10, Sieverding et al. (1979) show a typical pressure distribution around a turbine trailing edge in transonic conditions. The two outer pressure dips represent the low pressure just downstream of the expansion fans. Once the flow separates, a region of nearly constant pressure covers the majority of the trailing edge. It is generally accepted that this 


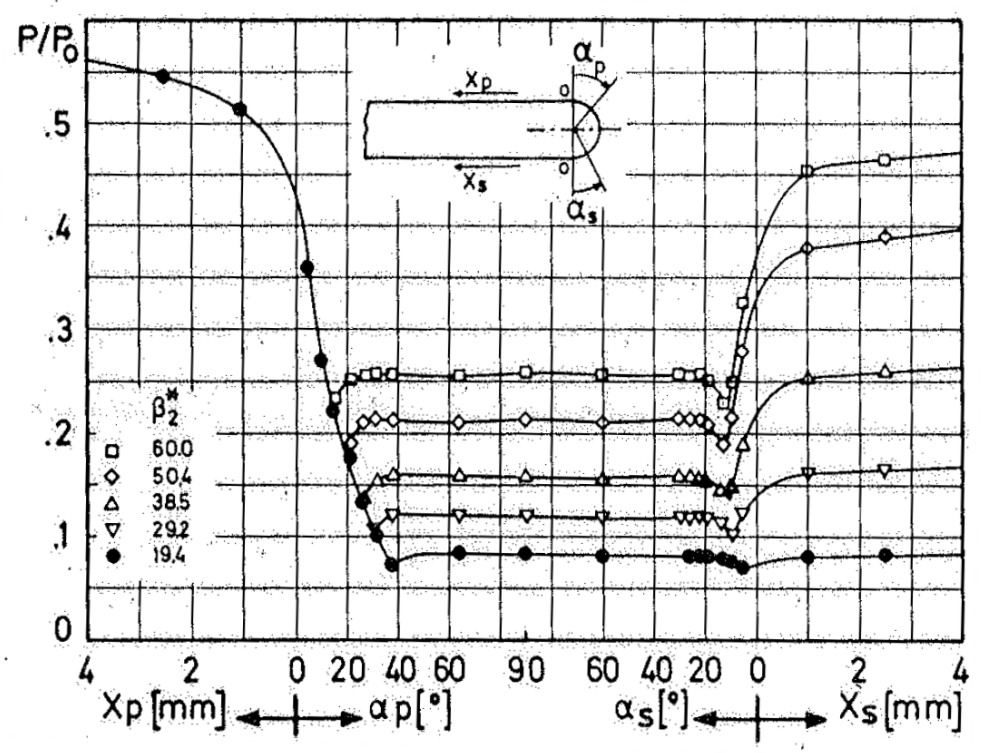

Figure 2.10: Typical Trailing Edge Pressure Distribution (Adapted from Sieverding et al., 1979

base pressure is associated with the strength of the trailing edge shock system. When the base pressure is lower, the separated flows expand further, resulting in stronger trailing edge shocks and a deeper wake.

Cascade testing has shown that the aerodynamic losses arising from flow around the trailing edge are significant. Using boundary layer traverse data to estimate the boundary layer losses of a transonic cascade, $\mathrm{Xu} \&$ Denton (1988) were able to subtract the boundary layer losses from the overall profile losses and isolate the trailing edge losses (the contribution of shock losses was not considered). The authors concluded that the trailing edge losses accounted for roughly $70 \%$ of the total profile losses, though the trailing edge blockage for the cascades tested was not provided. Using a similar approach, Mee et al. (1992) subtracted the boundary layer losses and the shock losses from the total profile losses of a particular transonic cascade. The remaining losses were caused by mixing and included the trailing edge losses. For this cascade, having a trailing edge blockage of $6.3 \%$ and operating at transonic Mach numbers, the mixing losses accounted for roughly $50 \%$ of the total profile losses. Given the apparent influence of trailing edge losses, understanding its driving factors is thus a worthwhile step in understanding the overall profile loss behaviour at transonic Mach numbers. 
In a thorough literature review on losses in turbomachinery, Denton (1993) develops an approximate expression for the trailing edge loss using a simplified control volume analysis and the incompressible flow assumption. The details of this derivation are given in Appendix B, and the final expression for trailing edge loss is

$$
Y_{T E}=\frac{\Delta P_{o}}{0.5 \rho V_{r e f}^{2}}=-\frac{C_{b, D} t}{w}+\frac{2 \theta}{w}+\left(\frac{\delta^{*}+t}{w}\right)^{2}
$$

where $\theta$ and $\delta^{*}$ are the boundary layer momentum thickness and displacement thickness respectively, $t$ is the trailing edge thickness, $w$ is the throat opening and $C_{b, D}$ is the base pressure coefficient defined by Denton:

$$
C_{b, D}=\frac{P_{b}-P_{r e f}}{0.5 \rho V_{r e f}^{2}}
$$

where $P_{b}$ is the base pressure, $P_{r e f}$ is a reference static pressure and $V_{r e f}$ is the flow velocity at the same reference point. Since the derivation assumes incompressible flow, $\rho$ is the constant density throughout the control volume. The first term on the right-hand side of Equation 2.6 represents the effect of a uniform base pressure applied across the trailing edge surface. The second term represents the losses from mixing out the boundary layer momentum thickness. The last term represents the losses due to the sudden expansion at the trailing edge, since this term contains the sum of the trailing edge thickness and the boundary layer displacement thickness.

Although base pressure is linked to trailing edge loss, the key parameters governing the behaviour of base pressure are not completely understood. Xu \& Denton (1988) argue that a lower base pressure is caused by flow entrainment in the shear layers next to the base region. The authors discuss the effect of the boundary layer characteristics on the base pressure, particularly the effect of shape factor $\left(H=\frac{\delta^{*}}{\theta}\right)$, which is a measure of the fullness of the boundary layer velocity profile. A lower shape factor describes a fuller velocity profile with greater fluid momentum in the boundary layer. If the boundary layer upstream of the trailing 


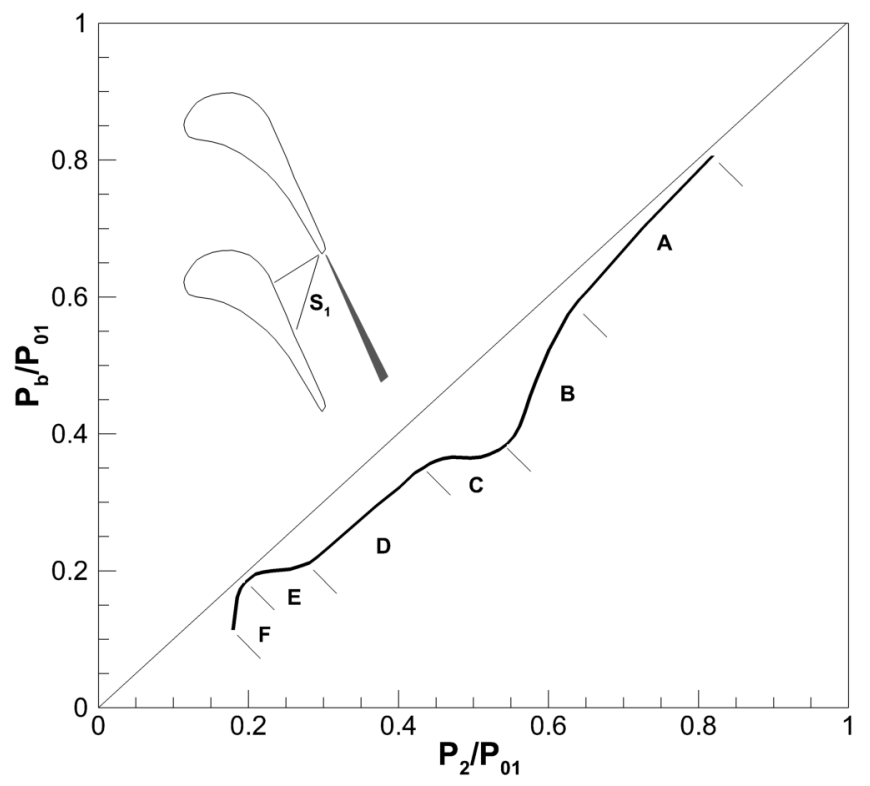

Figure 2.11: Behaviour of Base Pressure as a Function of Outlet Static Pressure (Adapted from Sieverding et al., 1979)

edge has a low shape factor, the separated shear layer which is formed from the boundary layer thus has a greater ability to entrain the base region fluid, leading to a lower base pressure.

Sieverding et al. (1979) performed an experimental study on base pressure and described the evolution of base pressure as a result of varying the ratio of downstream static pressure to upstream total pressure (and implicitly, the Mach number), as shown in Figure 2.11. Note that decreasing the ratio of outlet static pressure to upstream total pressure $\left(P_{2} / P_{o 1}\right)$ is equivalent to increasing the downstream Mach number. Segment A in Figure 2.11 corresponds to subsonic flow. At segment B, supersonic flow reaches the trailing edge and a normal shock $S_{1}$ forms at the trailing edge. As the downstream Mach number is increased further in segment $\mathrm{C}$, the suction side flow becomes completely supersonic and the trailing edge shock $S_{1}$ transitions from normal to oblique. Along segment $\mathrm{D}$, the increasingly oblique shock impinges on the suction surface further and further downstream. At segment E, the shock $S_{1}$ approaches the trailing edge and finally interacts with the wake in segment F. 


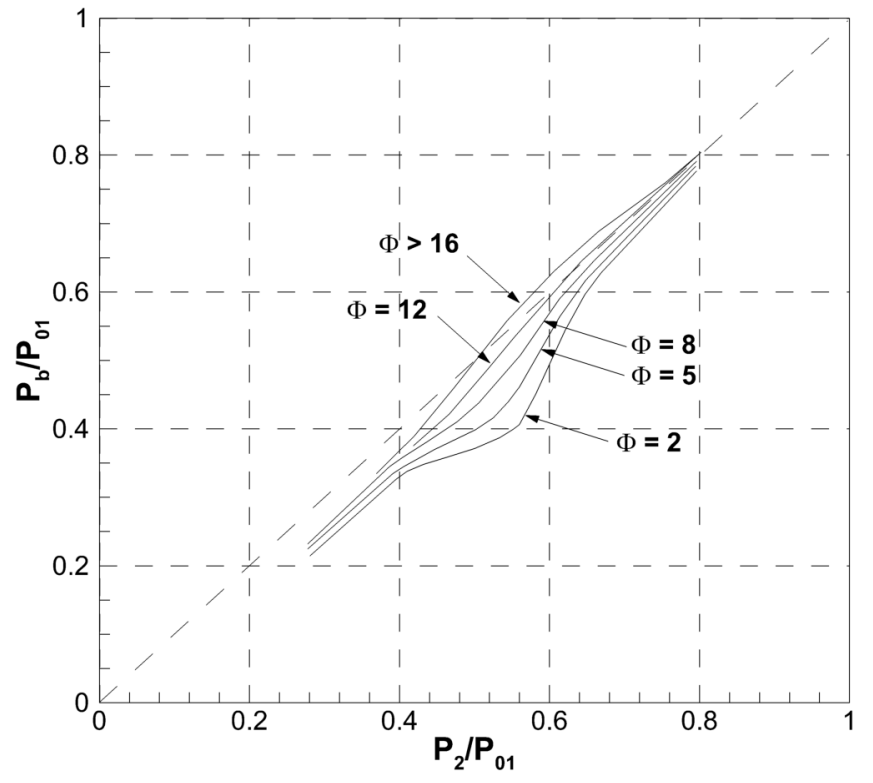

Figure 2.12: Base Pressure Correlation by Sieverding et al. (1979)

Despite identifying a general trend, Sieverding et al. (1979) found that the base pressure was difficult to predict due to the influence of airfoil geometry. However, it was noted by the authors that blades with high suction side curvature, particularly downstream of the throat, tend to maintain base pressures that are closer to the downstream static pressure. Sieverding et al. (1979) attempted to correlate this effect with the trailing edge wedge angle $\left(W_{e}\right)$ and unguided turning angle $\left(\theta_{u}\right)$ by defining the following parameter:

$$
\Phi=\frac{W_{e}+\theta_{u}}{2}
$$

The definitions of $W_{e}$ and $\theta_{u}$ are shown schematically in Figure 3.9 in the following chapter.

The Sieverding et al. correlation is shown in Figure 2.12. The correlation does offer some insight into base pressure behaviour, but it has been criticized for ignoring the effect of trailing edge blockage. As Sieverding himself admits, a more suitable correlation might include as a parameter the ratio of the boundary layer thickness to trailing edge thickness, though the data required to produce a trustworthy correlation did not (and may still not) exist. 
Given that the base pressure is so strongly linked with the trailing edge losses, understanding the flow mechanisms governing the base pressure behaviour may provide the key to understanding the transonic loss plateau described in Section 2.5.1. However, the main drivers of base pressure and loss in the transonic flow regime are still unclear, and are the subject of this study.

\subsubsection{Reynolds Number}

The Reynolds number represents the ratio of inertial forces to viscous forces, meaning that higher Reynolds number flows have less dominant viscous effects. In this study, the Reynolds number is defined based on the exit flow conditions and the true chord of the blade $\left(R e=\frac{\rho_{2} V_{2} C}{\mu}\right)$.

The Reynolds number affects the total pressure loss through its effect on the boundary layer properties. The effect of Reynolds number on losses has been well documented Baines et al., 1986; Hourmouziadis, 1989, Mayle, 1991; Ladwig \& Fottner, 1993). The Reynolds number has a strong influence on the location of transition from a laminar to a turbulent boundary layer. A turbulent boundary layer generates higher losses than a laminar boundary layer because the turbulent boundary layer's fuller velocity profile creates stronger shear forces at the blade surface. Despite generating higher frictional losses, a turbulent boundary layer can be desirable because it tends to delay the onset of separation that might otherwise occur in a laminar boundary layer.

In order to describe the effect of Reynolds number on losses, it is useful to describe the flow features in order of decreasing Reynolds number. A visual representation of these flow features is shown in Figure 2.13. At very high Reynolds numbers (a), the location of transition occurs very near the leading edge. A turbulent boundary layer covers most of the airfoil, causing higher losses due to the strong surface shear forces. With a sufficiently strong adverse pressure gradient near the trailing edge, turbulent separation can occur, causing additional mixing losses. As the Reynolds number decreases (b), the location of transition moves downstream. Losses decrease due to the larger proportion of laminar boundary layer. The losses normally reach a minimum just before the transition point reaches the point of 


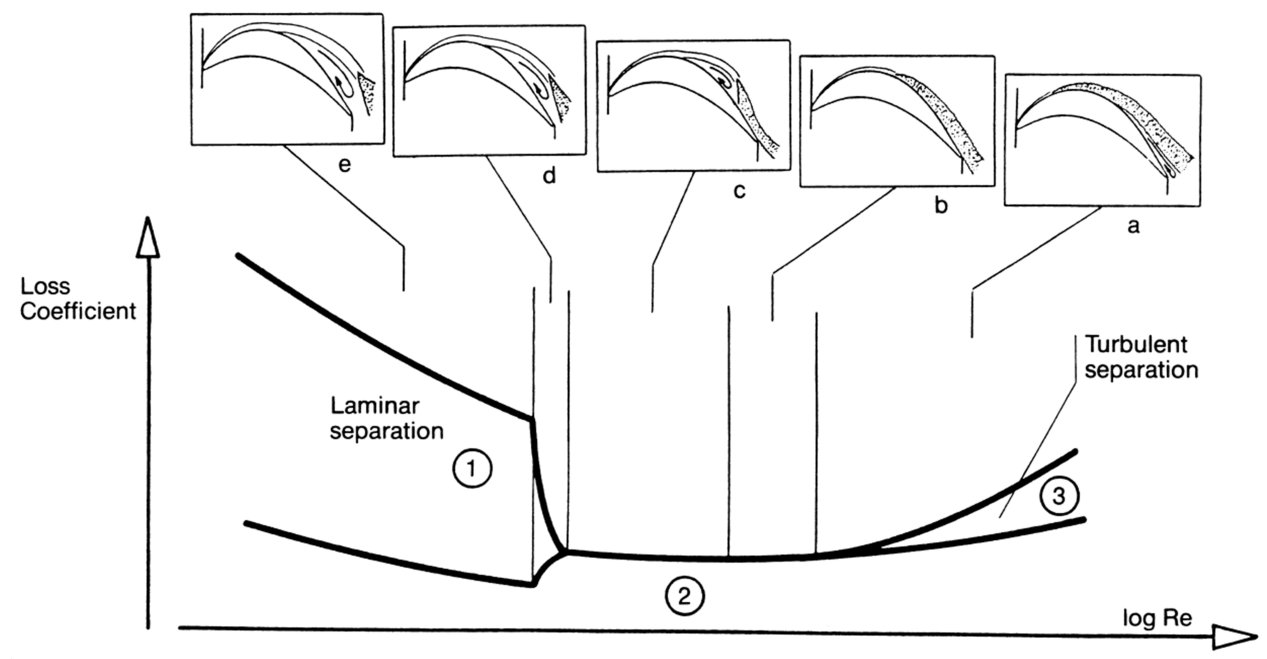

Figure 2.13: Effect of Reynolds Number on Profile Losses (Hourmouziadis, 1989)

laminar separation. In this condition, the laminar boundary layer extent is maximized and no separation occurs. Once the transition location passes the laminar separation point (c), the turbulent boundary layer no longer suppresses separation. A separation bubble forms and the flow reattaches near the transition location. Decreasing the Reynolds number further can have two outcomes. Along the upper curve, the separation bubble grows until it can no longer reattach $(d)$. Losses increase significantly due to the strong viscous mixing that occurs at the trailing edge and in the separated region. These losses continue to increase at lower Reynolds numbers if transition of the free shear layer occurs downstream of the trailing edge, completely clear of the airfoil (e). The lower curve represents the case where the Reynolds number becomes sufficiently low that the laminar boundary layer does not separate. This condition can be achieved on a carefully loaded airfoil with gentle adverse pressure gradients.

\subsubsection{Freestream Turbulence}

In order to quantify the turbulence observed in actual turbines, Porreca et al. (2007) performed measurements of turbulence intensity downstream of a rotor blade in a multistage axial turbine. At midspan, turbulence intensities as high as $9 \%$ were observed, for a blade Reynolds number of $R e=2 \times 10^{5}$. Similar measurements were made for a low pressure turbine by Binder et al. (1989) over a range of Reynolds numbers $\left(1.2 \times 10^{5} \leq R e \leq 4.93 \times 10^{5}\right)$. The 


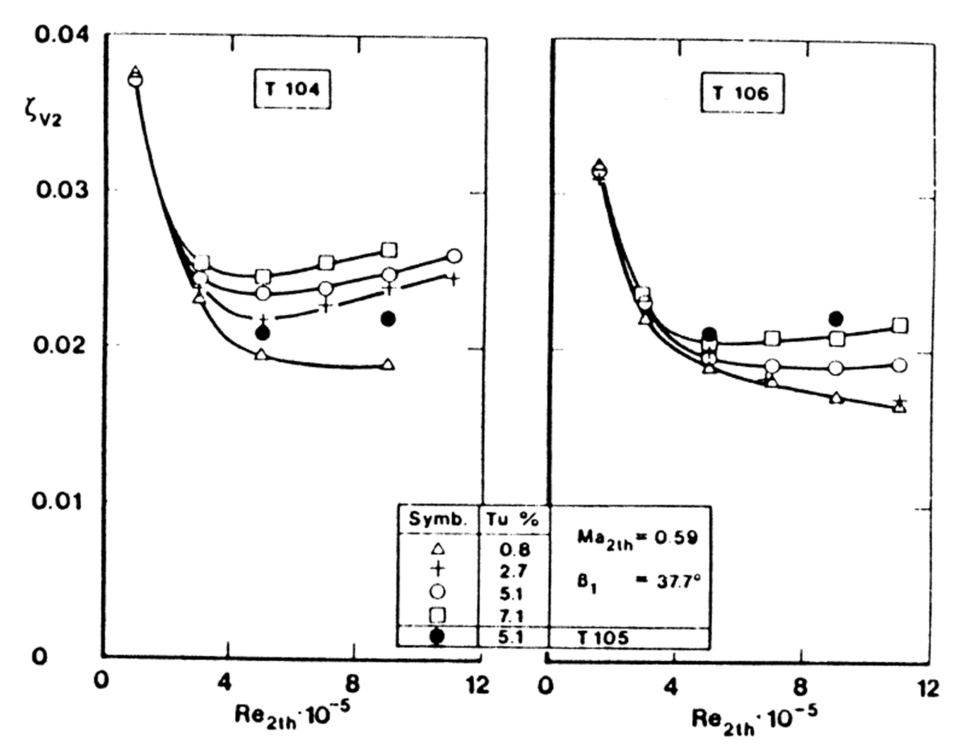

Figure 2.14: Effect of Turbulence Intensity on the Profile Losses of Three Turbine Cascades: T 104, T 105 and T 106 (Hoheisel et al., 1987)

highest values of turbulence intensity (8\%) occurred at the lowest Reynolds number. Even higher turbulence intensities generally occur in the high pressure turbine, as it is directly downstream of the combustor.

Given the large range of turbulence intensities encountered in practice, Hoheisel et al. (1987) investigated the combined effect of Reynolds number and turbulence intensity on blade profile losses. The results provided in Figure 2.14 show a sharp increase in losses at low Reynolds numbers, likely due to the formation of laminar separation bubbles. Little effect of turbulence intensity is observed at low Reynolds number. However, at higher Reynolds numbers, higher turbulence intensities are associated with greater losses. Mayle (1991) explains that higher freestream turbulence intensity causes transition to occur at a lower Reynolds number, causing the transition point to move upstream on the blade suction surface. The increased turbulence level in the boundary layer has the effect of increasing the wall shear stress, thus increasing the loss production in the boundary layer. 


\subsubsection{Axial Velocity Density Ratio (AVDR)}

The axial velocity density ratio (AVDR) is defined as follows:

$$
A V D R=\frac{\left(\rho V_{x}\right)_{2}}{\left(\rho V_{x}\right)_{1}}=\frac{\left(\frac{\dot{m}}{A}\right)_{2}}{\left(\frac{\dot{m}}{A}\right)_{1}}=\frac{\int_{0}^{1}\left(\rho_{2} V_{x, 2}\right)_{M S} \mathrm{~d}(y / s)}{\int_{0}^{1}\left(\rho_{1} V_{x, 1}\right)_{M S} \mathrm{~d}(y / s)}
$$

where $V_{x}$ is the axial velocity and $\left(\rho V_{x}\right)$ is the mass-averaged value for a thin streamtube at midspan extending over one blade pitch. The integrated expression on the right-hand side is the full definition of AVDR, but the other forms are useful for explaining the physical significance of AVDR. Recalling the expression for mass flow rate $\left(\dot{m}=\rho V_{x} A\right)$, the AVDR is the ratio of the mass flow per unit area at the outlet relative to the inlet. The AVDR is a measure of the average flow convergence $(A V D R>1.0)$ or divergence $(A V D R<1.0)$ that the flow experiences at midspan. For this reason, $A V D R=1.0$ is a necessary condition for flow two-dimensionality at midspan. However, for the blade surface flow to be truly twodimensional, there must be no local region of convergence or divergence across the blade pitch. Since the AVDR is averaged in the pitchwise direction, a value of AVDR $=1.0$ is a necessary but insufficient condition for flow two-dimensionality at midspan.

In a planar cascade where the endwalls are parallel, the flow convergence or divergence is a consequence of the blockage associated with the displacement thickness of the endwall boundary layers. The blade-to-blade pressure distribution tends to thicken the endwall boundary layer near the blade suction-side and to thin it near the blade pressure-side. As a result, the suction-side midspan flow may experience convergence at the same time that the pressure-side midspan flow experiences divergence, even though the average convergence or divergence may be zero $(A V D R=1.0)$. High aspect ratio blades minimize the effect of endwall flows on the midspan flow convergence. For low aspect ratio blades $(<3.0)$, flow two-dimensionality is more difficult to achieve.

A study by Rodger et al. (1992) examined the effect of flow convergence and divergence on profile losses in a turbine cascade at different incidence angles, as shown in Figure 2.15a. Given that the study is for incompressible flow, the AVR (Axial Velocity Ratio) was given because density changes through the cascade were negligible. From Figure 2.15a, the profile 


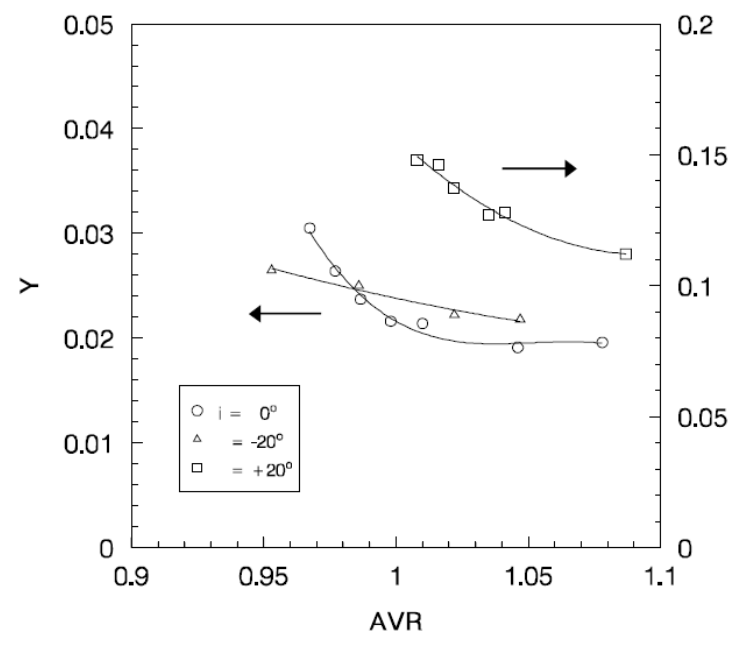

(a) Effect of AVR on Profile Losses

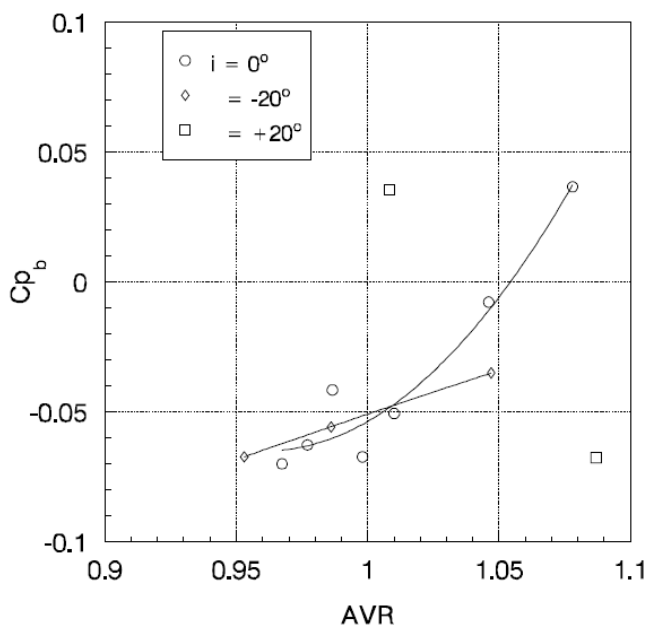

(b) Effect of AVR on Base Pressure

Figure 2.15: Effect of AVR on Profile Losses and Base Pressure, Provided for Three Values of Incidence Angle (Rodger et al., 1992)

losses at zero incidence angle increase in the case of diverging flow $(A V R<1.0)$. Rodger et al. (1992) also found that diverging flow caused a decrease in the base pressure, as shown in Figure 2.15b, Given that low base pressures are generally associated with higher trailing edge losses, the decreasing base pressure observed with decreasing AVR likely contributed to the increase in losses.

\subsection{Correlations for Profile Losses at Design Incidence}

\subsubsection{Introduction}

In the early stages of turbine design, it is necessary to estimate the efficiency of a new turbine in order to know that its final power output meets the design requirements. Empirical loss correlations allow the designer to estimate turbine efficiency using a limited number of geometric and flow parameters. Given that loss correlations are based on actual turbine performance, they must be updated periodically to reflect improvements in current technology. The following section describes a well known loss prediction system, the Ainley \& Mathieson (1951) correlation. Subsequently, several authors suggested improvements to the correlation, 
including Dunham \& Came (1970) and Kacker \& Okapuu (1982). The last section will also briefly cover some alternatives to the Ainley \& Mathieson loss correlation.

\subsubsection{Ainley and Mathieson Loss Correlation}

One of the most widely used axial turbine loss systems was proposed by Ainley \& Mathieson (1951). The correlation provides predictions of loss at both design and off-design incidence angles, for profile losses, secondary losses and tip leakage losses. The following review will only cover profile loss prediction methods at design incidence, since these are the subject of the current study.

The Ainley \& Mathieson system provides profile loss data for two extremes of flow acceleration for a given outlet flow angle $\left(\alpha_{2}\right)$. The nozzle blade $\left(\beta_{1}=0^{\circ}\right)$ provides maximum flow acceleration, while the impulse blade $\left(\beta_{1}=\alpha_{2}\right)$ provides no flow acceleration. These profile loss data, shown in Figures 2.16a and 2.16b, are given for a range of pitch to chord ratios $(s / c)$. The predicted values for the blades examined in this thesis are also indicated. In practice, the blade inlet angle is typically an intermediate value between nozzle and impulse blades. For these cases, the Ainley and Mathieson system uses a weighted average for the profile losses, as follows:

$$
Y_{P, A M}=\left\{Y_{P,\left(\beta_{1}=0\right)}+\left(\frac{\beta_{1}}{\alpha_{2}}\right)^{2}\left[Y_{P,\left(\beta_{1}=\alpha_{2}\right)}-Y_{P,\left(\beta_{1}=0\right)}\right]\right\}\left(\frac{t_{\max } / C}{0.2}\right)^{\beta_{1} / \alpha_{2}}
$$

In Equation 2.10, $Y_{P,\left(\beta_{1}=0\right)}$ and $Y_{P,\left(\beta_{1}=\alpha_{2}\right)}$ are the profile loss coefficients for nozzle and impulse blades, respectively, taken from Figures $2.16 \mathrm{a}$ and $2.16 \mathrm{~b}$.

The profile loss data provided in Figures 2.16a and 2.16b are for blades with a maximum thickness of $20 \%$ blade chord and with a trailing edge thickness $(t)$ of $2 \%$ of the blade pitch. The $t_{\max } / C$ term in Equation 2.10 provides a correction for blades with different maximum thicknesses. For blades with different trailing edge thicknesses, the Ainley \& Mathieson system offers a correction in the form of a multiplying factor, obtained from Figure 2.17.

Most of the data presented by Ainley \& Mathieson were taken at a Reynolds number of $2 \times 10^{5}$. For blades operating at different Reynolds numbers, the following correction is 


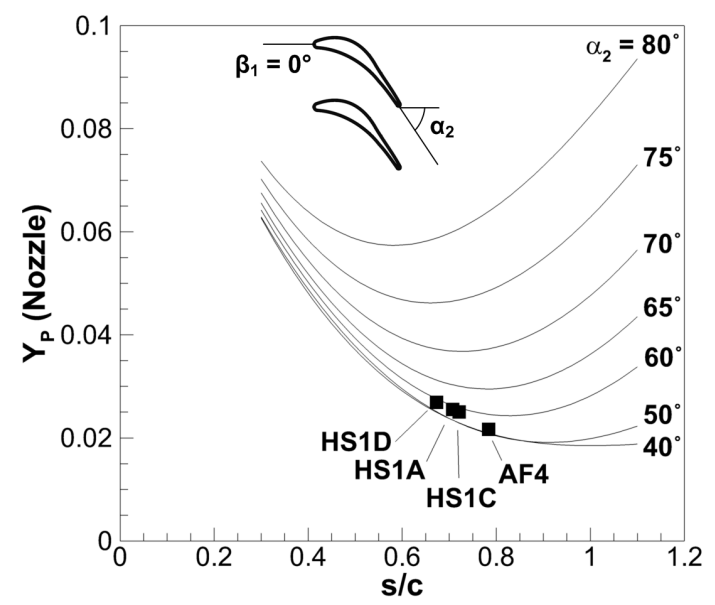

(a) Nozzle Blades $\left(\beta_{1}=0^{\circ}\right)$

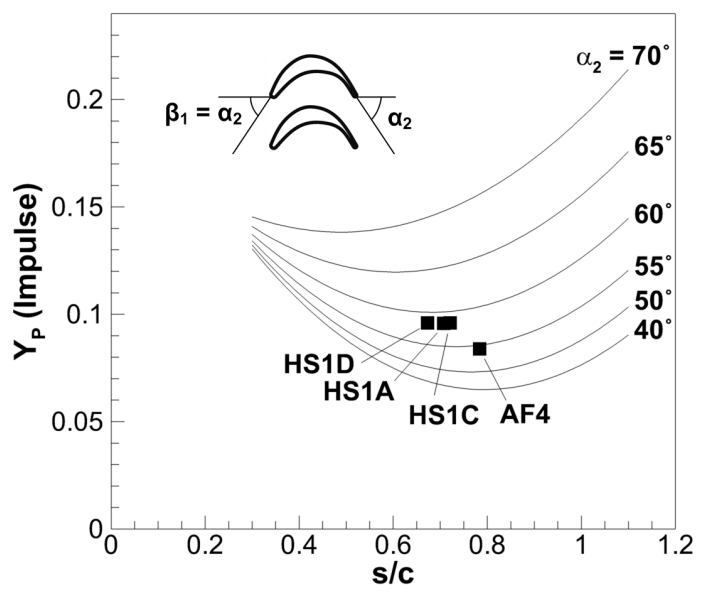

(b) Impulse Blades $\left(\beta_{1}=\alpha_{2}\right)$

Figure 2.16: Profile Loss Coefficients Predicted by the Ainley \& Mathieson (1951) Correlation, Including the Predictions for the Four Cascades Used in this Study

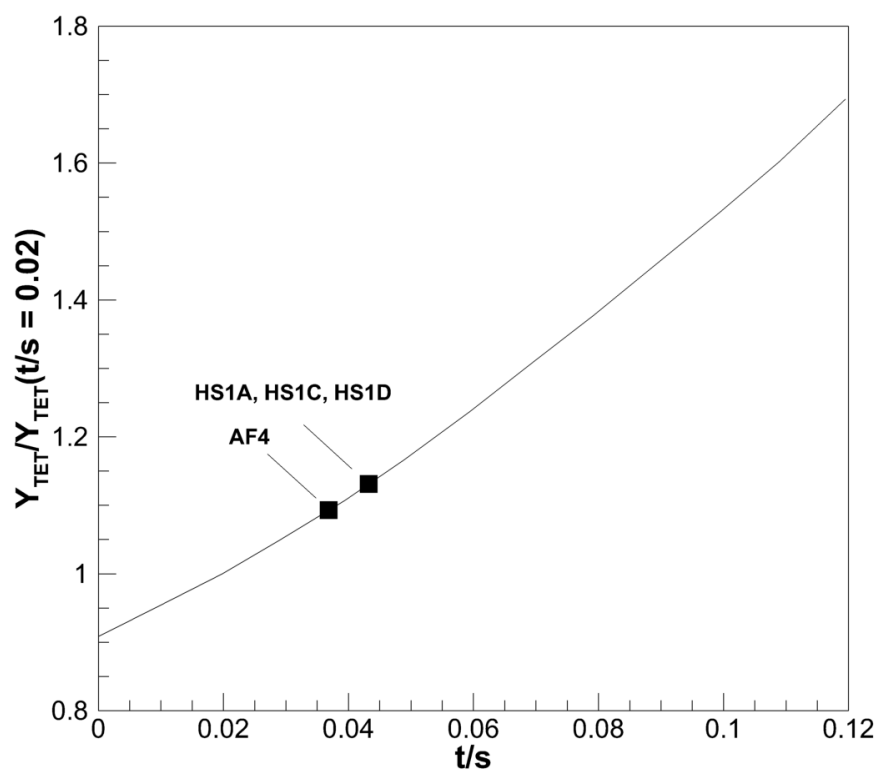

Figure 2.17: Correction Factor for Trailing Edge Thickness (t), Non-Dimensionalized by the Blade Pitch (s) Ainley \& Mathieson, 1951) 
applied to the overall turbine isentropic efficiency:

$$
1-\eta_{\text {corrected }}=(1-\eta)\left(\frac{R e}{2 \times 10^{5}}\right)^{-1 / 5}
$$

where $\eta$ is the overall turbine efficiency. This correction is cumbersome to apply, as it is necessary to first obtain the overall total pressure loss coefficient of the blade assuming the nominal Reynolds number of $2 \times 10^{5}$, and then to convert the total pressure loss into an efficiency value. The correction can then be applied to obtain the overall turbine efficiency at a different Reynolds number.

The profile loss data were collected at a Mach number of less than $M_{2}=0.6$. Ainley \& Mathieson offer no correction for the effect of Mach number.

\subsubsection{Dunham and Came Loss Correlation}

Based on performance data from 16 more recent turbine designs, the Dunham \& Came (1970) loss system provides a modified correlation based on Ainley \& Mathieson (1951). In particular, the new Ainley \& Mathieson, Dunham \& Came (AMDC) loss system offers an improved correction for the Reynolds number and a new correction for the Mach number.

The Reynolds number correction is modified by applying it directly to profile losses and secondary losses instead of efficiency. In order to account for the effect of Mach number, a compressibility correction $k_{C}$ is introduced as a multiplier to the profile losses when the outlet Mach number $\left(M_{2}\right)$ exceeds unity, as shown in Figure 2.18. The compressibility correction is defined as:

$$
k_{C}=1+60\left(M_{2}-1\right)^{2}
$$

As described in Section 2.5.1, the effect of Mach number on losses is complex. As the Mach number is increased through the transonic regime, losses do not simply increase in a parabolic trend as indicated by Dunham \& Came (1970). The profile losses sometimes reach a plateau or even reduce somewhat at low supersonic Mach numbers before increasing again at higher Mach numbers. While the Dunham \& Came system offers an improvement over the Ainley \& Mathieson system, the physics of the transonic loss behaviour are still not adequately 


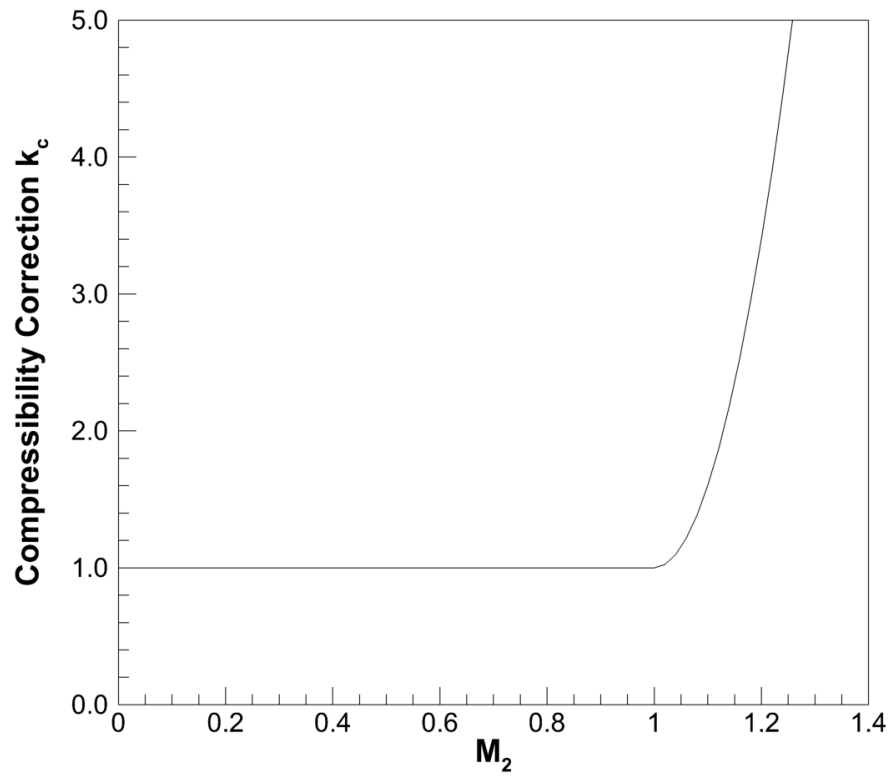

Figure 2.18: Compressibility Correction Factor (Dunham \& Came, 1970)

captured. This loss behaviour is not well understood, even to this day, and is the subject of the current investigation.

\subsubsection{Kacker and Okapuu Loss Correlation}

Kacker \& Okapuu (1982) proposes additional improvements to the Ainley \& Mathieson, Dunham \& Came loss system for blades operating at design incidence. Kacker \& Okapuu divide the losses into components as follows:

$$
Y_{\text {Total }}=Y_{P} f(R e)+Y_{S}+Y_{T E T}^{\prime}+Y_{T C}
$$

where $Y_{P}, Y_{S}, Y_{T E T}^{\prime}$ and $Y_{T C}$ are the total pressure loss coefficients for profile, secondary, trailing edge and tip clearance losses, respectively. $f(R e)$ is a Reynolds number correction factor, applied only to the profile losses. 
In order to predict profile losses, Kacker \& Okapuu apply modifications to the profile loss predictions given by the AMDC system, as follows.

$$
Y_{P}=0.914\left(\frac{2}{3} Y_{P, A M D C} K_{P}+Y_{S H O C K}\right)
$$

As seen in Equation 2.13, the Kacker \& Okapuu system recognizes the trailing edge losses as being a separate loss component from the profile losses. The factor of 0.914 is employed to account for the removal of trailing edge losses from the AMDC profile loss term. The value of 0.914 is the value of the original $Y_{T E T}$ multiplier in the AMDC system when the trailing edge thickness is set to zero. The factor of $2 / 3$ was used by Kacker \& Okapuu to reflect the reductions in turbine profile losses obtained by improved design methods since the original data were collected by Ainley \& Mathieson.

The $Y_{P, A M D C}$ term is simply the weighted average between nozzle and impulse blades used in the AMDC system, as obtained from the following modified version of Equation 2.10:

$$
Y_{P, A M D C}=\left\{Y_{P,\left(\beta_{1}=0\right)}+\left|\frac{\beta_{1}}{\alpha_{2}}\right|\left(\frac{\beta_{1}}{\alpha_{2}}\right)\left[Y_{P,\left(\beta_{1}=\alpha_{2}\right)}-Y_{P,\left(\beta_{1}=0\right)}\right]\right\}\left(\frac{t_{\max } / C}{0.2}\right)^{\beta_{1} / \alpha_{2}}
$$

The absolute value sign added to the $\left|\beta_{1} / \alpha_{2}\right|$ ratio by Kacker \& Okapuu allows the system to accommodate negative inlet metal angles $\left(\beta_{1}\right)$.

Kacker \& Okapuu also introduced new corrections for compressibility effects. The $K_{P}$ multiplier used in Equation 2.14 accounts for the effects of channel acceleration, and is defined as follows:

$$
K_{P}=1-K_{2}\left(1-K_{1}\right)
$$

where $K_{1}$ and $K_{2}$ are given by:

$$
K_{1}=1-1.25\left(M_{2}-0.2\right) \text { for } M_{2}>0.2
$$

and

$$
K_{2}=\left(\frac{M_{1}}{M_{2}}\right)^{2}
$$


The $Y_{S H O C K}$ term in Equation 2.14 is employed to account for leading edge shocks which typically form at the blade hub. The expression derived by Kacker \& Okapuu for $Y_{S H O C K}$ is the following:

$$
Y_{\text {SHOCK }}=\left(\frac{\Delta P_{o}}{q_{1}}\right)\left(\frac{P_{1}}{P_{2}}\right) \frac{1-\left(1+\frac{\gamma-1}{2} M_{1}^{2}\right)^{\frac{\gamma}{\gamma-1}}}{1-\left(1+\frac{\gamma-1}{2} M_{2}^{2}\right)^{\frac{\gamma}{\gamma-1}}}
$$

For $M_{2} \geq 1.0$, Kacker \& Okapuu multipliy the profile losses with the same supersonic drag rise factor as in the AMDC system, renaming the factor CFM:

$$
C F M=1+60\left(M_{2}-1\right)^{2}
$$

Kacker \& Okapuu admit that the CFM is unsatisfactory, and does not account for the supersonic drag rise associated with limit load, which depends on outlet flow angle.

Kacker \& Okapuu improve upon the AMDC Reynolds number correction by basing the Reynolds number influence on skin friction models. The improved correlation considers three ranges: laminar, transitional and turbulent flow. The corresponding Reynolds number corrections are the following:

$$
f(R e)= \begin{cases}\left(\frac{R e}{2 \times 10^{5}}\right)^{-0.4} & \text { for } R e \leq 2 \times 10^{5} \\ 1.0 & \text { for } 2 \times 10^{5}<R e<1 \times 10^{6} \\ \left(\frac{R e}{1 \times 10^{6}}\right)^{-0.2} & \text { for } R e \geq 1 \times 10^{6}\end{cases}
$$

For the transitional range, $2 \times 10^{5}<R e<1 \times 10^{6}$, Kacker \& Okapuu offer no correction for the effect of Reynolds number, as no clear dependence on Reynolds number was observed.

Since the trailing edge losses have been separated from profile losses, Kacker \& Okapuu propose a new trailing edge loss correlation. The trailing edge losses take the form of an energy loss coefficient, obtained using a weighted average between the equivalent nozzle and impulse blade configurations. Kacker \& Okapuu use the ratio of trailing edge thickness (t) to throat opening (o) as a parameter, unlike Ainley \& Mathieson, who non-dimensionalize by the blade pitch (s). The nozzle and impulse blade energy loss coefficients are drawn from 


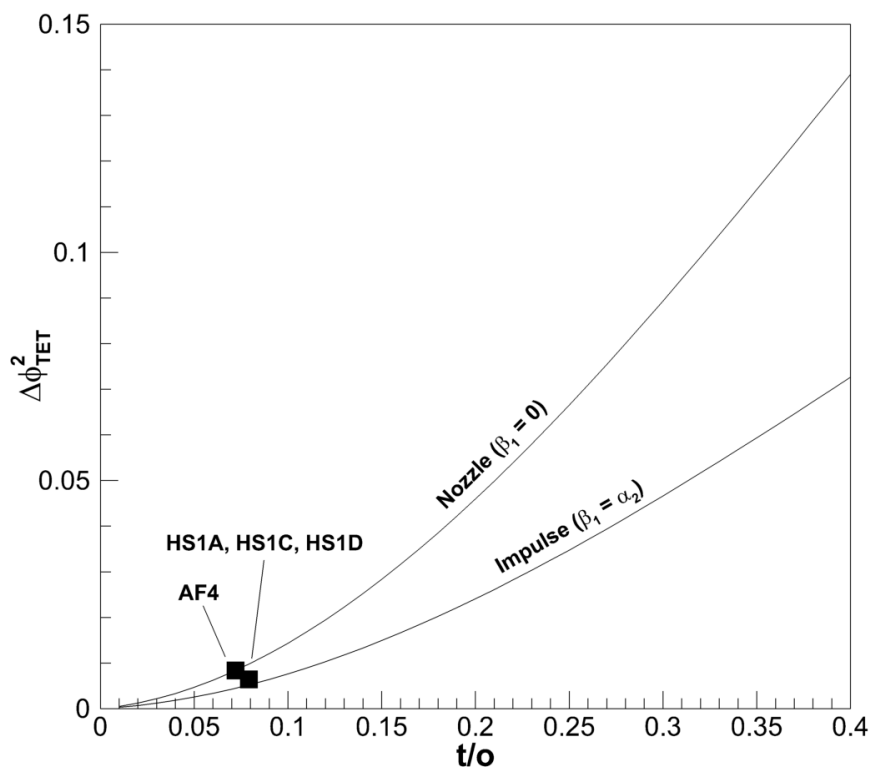

Figure 2.19: Trailing Edge Losses from Kacker \& Okapuu (1982), Expressed as Energy Loss Coefficient

Figure 2.19, and a weighted average for intermediate geometries is then given by the following equation:

$$
\Delta \phi_{T E T}^{2}=\Delta \phi_{T E T\left(\beta_{1}=0\right)}^{2}+\left|\frac{\beta_{1}}{\alpha_{2}}\right|\left(\frac{\beta_{1}}{\alpha_{2}}\right)\left[\Delta \phi_{T E T\left(\beta_{1}=\alpha_{2}\right)}^{2}-\Delta \phi_{T E T\left(\beta_{1}=0\right)}^{2}\right]
$$

In order to convert the energy loss coefficient from Equation 2.21 into a total pressure loss coefficient, the following expression is provided by Kacker \& Okapuu:

$$
Y_{T E T}^{\prime}=\frac{\left[1-\frac{\gamma-1}{2} M_{2}^{2}\left(\frac{1}{1-\Delta \phi_{T E T}^{2}}-1\right)\right]^{-\frac{\gamma}{\gamma-1}}-1}{1-\left(1+\frac{\gamma-1}{2} M_{2}^{2}\right)^{-\frac{\gamma}{\gamma-1}}}
$$

Although the energy loss coefficient provided by Kacker \& Okapuu is independent of the outlet Mach number, the conversion to total pressure loss coefficient incorporates the Mach 


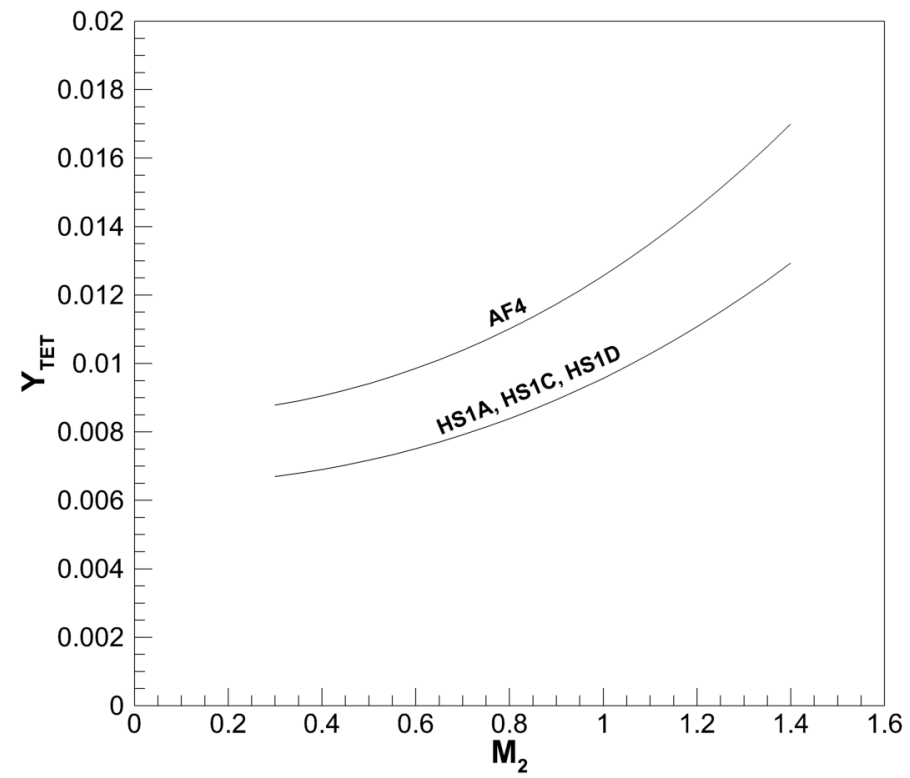

Figure 2.20: Trailing Edge Total Pressure Loss Coefficient of the Studied Airfoils, as Predicted by Kacker \& Okapuu (1982)

number. For the airfoils under study, the resulting trailing edge losses are shown in Figure2.20 as a function of the outlet Mach number.

In order to visualize the final result of the Kacker \& Okapuu loss correlation, the losses for the cascades examined in the present study are predicted. The prediction is made at blade midspan, such that the contributions from secondary and tip-leakage losses are excluded. The contribution from shocks at the blade hub $\left(Y_{S H O C K}\right)$ is also excluded. The remaining contributions are the profile and trailing edge losses, calculated over a wide range of Mach number. This loss prediction is shown in Figure 2.21. As the outlet Mach number is increased beyond sonic conditions, the losses are strongly influenced by the parabolic increase of the CFM multiplier. Once again, comparing this behaviour to the wind tunnel measurements presented in Figure 2.8, the prediction by Kacker \& Okapuu does not reflect the profile loss "plateau" observed in turbine cascades at supersonic Mach numbers. 


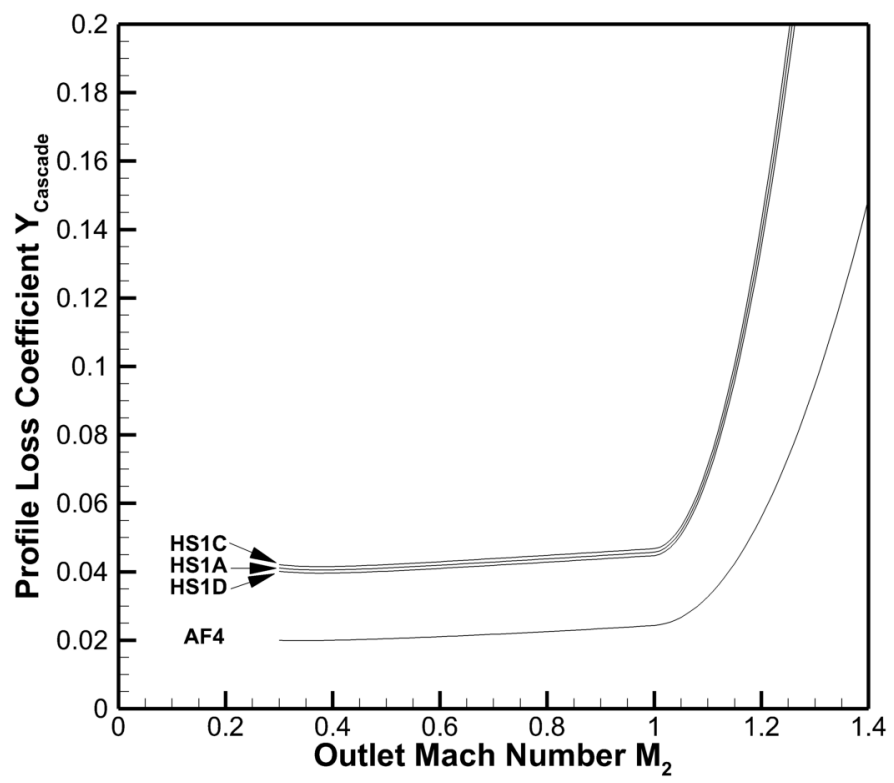

Figure 2.21: Losses Predicted by the Kacker \& Okapuu (1982) Correlation at Midspan for the Cascades Used in the Current Study

\subsubsection{Alternatives to the Ainley and Mathieson Family of Loss Correlations}

While the Ainley \& Mathieson (1951) family of correlations is commonly used, other formulations of loss predictions have been proposed. Denton (1973) performed a comparison of seven profile loss correlations: Ainley \& Mathieson (1951), Baljé \& Binsley (1968), Bolte (1964), Craig \& Cox (1971), Deich (1953), Denton (1971) and Traupel (1966). The predicted profile losses from these correlations are shown in Figure $2.22 \mathrm{a}$ for blades with an axial inlet metal angle, and in Figure $2.22 \mathrm{~b}$ for blades with an inlet metal angle of $60^{\circ}$. The blades are considered to have optimum pitch to chord ratio $(s / c)$, and are operated at a Reynolds number of $2 \times 10^{5}$ and at a low subsonic Mach number. There is a large amount of scatter in these figures, particularly in Figure 2.22b, where the maximum difference between the predictions is on the order of $50 \%$ of their mean value. Denton (1973) also compared the profile loss predictions with a total of 79 experimental cascade results. All methods were generally found to provide poor agreement between the predicted and experimental values of profile loss, with standard deviations on the order of $35 \%$ of the profile loss values. 


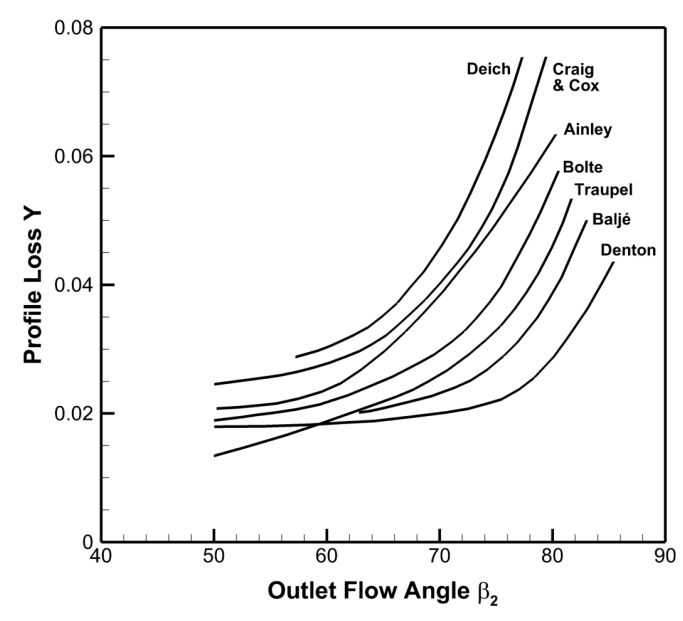

(a) Nozzle Blades $\left(\beta_{1}=0^{\circ}\right)$

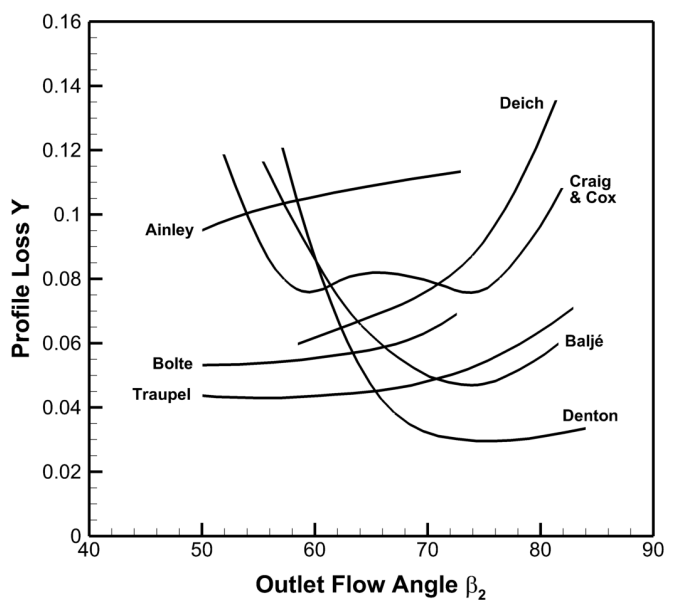

(b) Blades with $\beta_{1}=60^{\circ}$

Figure 2.22: Profile Loss Coefficients Predicted by Seven Profile Loss Correlations (Adapted from Denton, 1973)

Denton explained that these correlation methods are based on the philosophy that profile losses are mainly a function of the flow angles and basic geometric parameters, rather than the detailed blade curvature. Denton (1973) reasoned that the poor agreement suggests that this philosophy might not be entirely correct. While some mechanisms of profile loss generation are undoubtedly dependent on the detailed blade geometry, these simplified loss correlations continue to be used in the early stages of design for their immediate feedback on the merits of a turbine blade design. Presumably, the Ainley \& Mathieson (1951) correlation has endured because turbine designers have found the approach to reasonably capture the influence of basic geometric parameters, in part resulting from the repeated efforts to improve and modernize the correlation by authors such as Dunham \& Came (1970) and Kacker \& Okapuu (1982).

\subsection{Summary}

The Kacker \& Okapuu (1982) loss correlation could be improved in its treatment of profile losses at transonic and supersonic Mach numbers. The next step is to identify what physical mechanisms are responsible for the loss plateau. The expression provided by Denton (1993) in Equation 2.6 suggests that trailing edge losses are a function of the base pressure and the 
boundary layer parameters at the trailing edge. From the Sieverding et al. (1979) correlation, the base pressure is related to the suction side curvature downstream of the throat (unguided turning). While these authors offer insight on the important flow variables, a better understanding of the flow mechanisms governing profile losses at transonic and supersonic Mach numbers is needed before creating a reliable loss correlation.

The purpose of the current investigation is to elucidate the flow mechanisms that drive the profile losses in the transonic and supersonic regimes. Using the Pratt \& Whitney Canada High-Speed Wind Tunnel at Carleton University, new experimental profile loss data are offered to supplement the existing data in the literature. Numerical simulations are performed to replicate wind tunnel data, allowing a closer look at the flow inside the blade passages and near the blade surfaces. Chapter 3 describes the high-speed wind tunnel facilities used to generate the experimental data. Chapter 4 describes the methods used to collect and process the data, and Chapter 5 provides the numerical methods used for the simulations. 


\section{Chapter 3}

\section{Experimental Apparatus}

\subsection{Introduction}

The current chapter presents the Pratt \& Whitney Canada High-Speed Wind Tunnel Laboratory. First, the wind tunnel hardware is described. Secondly, the instrumentation is discussed, including the three-hole probe and static probe used for wake measurements. The detailed geometry of the cascades is then provided.

\subsection{Pratt \& Whitney Canada High-Speed Wind Tunnel}

\subsubsection{Introduction}

The experimental data collected during the present study were obtained using the Pratt \& Whitney Canada High-Speed Wind Tunnel Laboratory at Carleton University (Figure 3.1. The wind tunnel was originally constructed at McMaster University in Hamilton and was recommissioned at Carleton University by Jeffries (1994). It is an intermittent, blow-down type wind tunnel used for transonic turbine cascade research. The wind tunnel operates by filling large air storage tanks using a compressor. The tanks are then discharged at a controlled Mach number through the test section, which contains the cascade of turbine blades and all required instrumentation. 


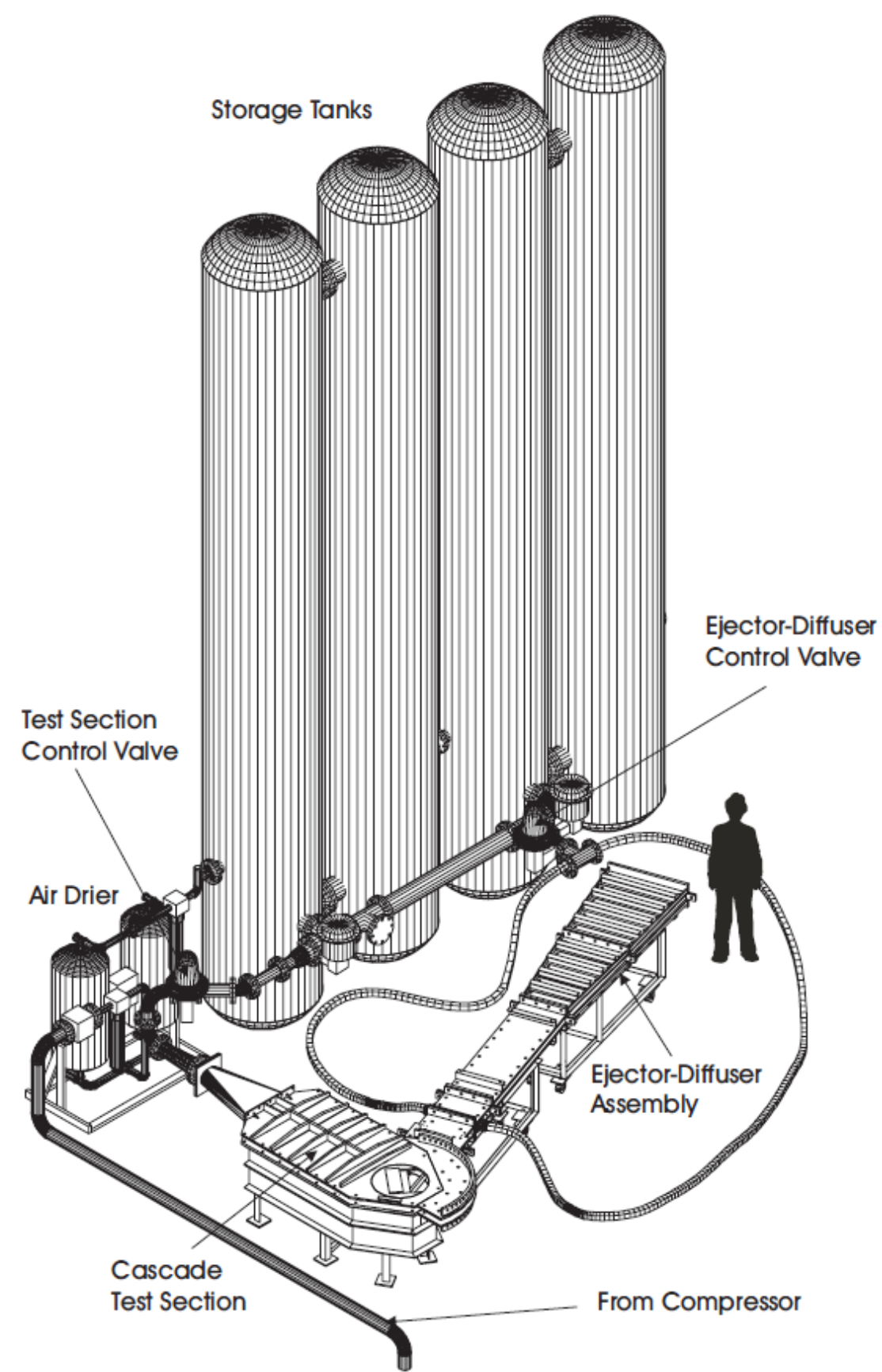

Figure 3.1: Pratt \& Whitney Canada High-Speed Wind Tunnel at Carleton University (Corriveau, 2005)

\subsubsection{Compressor}

Air is supplied to the wind tunnel storage tanks by a Broom-Wade model VC500 two-stage intercooled positive-displacement compressor. The compressor is shown in Figure 3.2. The 
compressor is powered by a $100 \mathrm{hp} \mathrm{AC} \mathrm{three-phase} \mathrm{electric} \mathrm{motor.} \mathrm{The} \mathrm{compressor} \mathrm{delivers}$ a volume flow rate of roughly $14.2 \mathrm{~m}^{3} / \mathrm{min}$ (500 S.C.F.M). The compressor provides air to the storage tanks up to a maximum pressure of about $690 \mathrm{kPa}(100 \mathrm{psi}(\mathrm{g}))$, which takes approximately 20 minutes starting from atmospheric pressure. Air leaving the compressor is passed through a reverse flow aftercooler to lower the air temperature from about $100^{\circ} \mathrm{C}$ to about $27^{\circ} \mathrm{C}$. This process generates some condensation. Therefore the air is passed through a water separator to remove excess moisture.

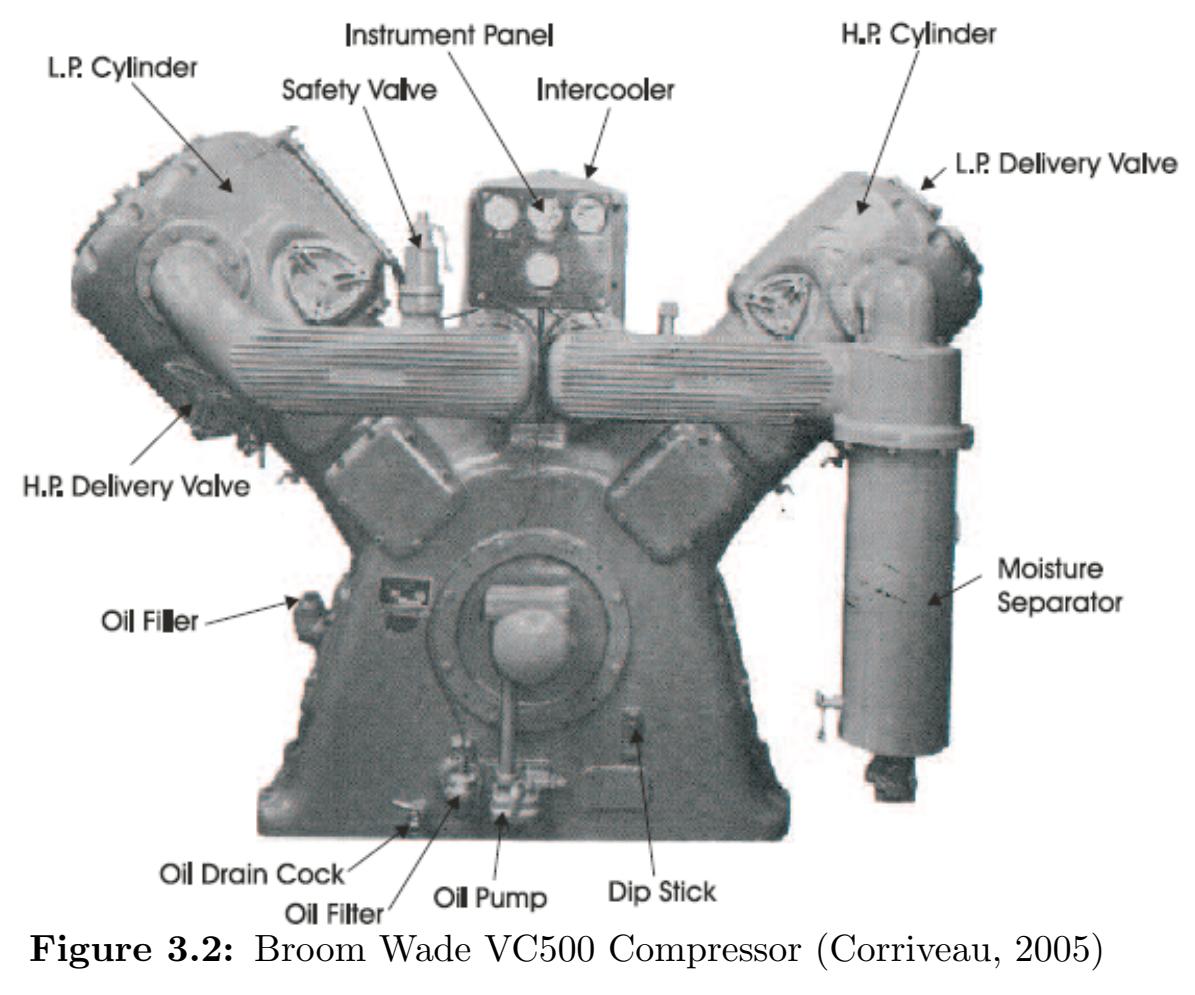

\subsubsection{Air Dryer}

When the wind tunnel is operating, the air expands in the storage tanks and through the cascade test section. The temperature of the air in the storage tanks, and by extension the stagnation temperature of the air in the test section, is thus continuously dropping during a run. Additionally, as the air is accelerated through the test section the static temperature 
decreases. Given the low temperatures in the test section, it is possible for condensation and even ice formation to occur.

For this reason, the air is dried prior to entering the storage tanks using a Comp-Air Kellogg model CDH520 regenerative desiccant dryer. The system is composed of two tanks containing a desiccant material. The tanks alternate modes: while one tank is used to dry the air from the compressor, some high pressure air is used to flush and regenerate the desiccant in the other tank. This drying system lowers the dew point of the air to about $-45^{\circ} \mathrm{C}$, which prevents the formation of condensation up to Mach numbers of about 1.2.

\subsubsection{Air Storage Tanks}

The Pratt \& Whitney Canada high speed wind tunnel employs four large pressure vessels, each having a volume of roughly $6.5 \mathrm{~m}^{3}$. The total internal volume of stored air is about $26 \mathrm{~m}^{3}$. The arrangement of four tanks can be subdivided into sets of two, to be used separately for alternative test setups. However, for the purposes of this research, all four tanks were used simultaneously in parallel.

\subsubsection{Shutoff and Control Valves}

The wind tunnel has two flow paths: the main test section flow path, and the auxiliary system flow path. The auxiliary airflow can be used to operate an ejector system attached to the wind tunnel outlet, which allows independent control of the Reynolds number. The auxiliary system is also used to provide airflow to the in-house probe calibration rig. The main and auxiliary flow paths are controlled independently. In both cases, air first passes through a shutoff valve, which is kept closed for safety when the wind tunnel is not operating. Downstream, a control valve regulates the total pressure of the air supply using the control system described in Section 4.2. The specifications for the valves, actuators and positioners are provided in Table 3.1 . 
Table 3.1: Specifications for the Valve Used in the High-Speed Wind Tunnel

\begin{tabular}{cllll}
\hline & Function & \multicolumn{1}{c}{ Valve } & \multicolumn{1}{c}{ Actuator } & Positioner \\
\hline \hline $\begin{array}{c}\text { Main Test } \\
\text { Section }\end{array}$ & Shut-Off & $\begin{array}{l}\text { 6" N.J. }{ }^{1} 815 \mathrm{~L} \\
\text { Butterfly }\end{array}$ & N.J. QP3C-M & - \\
\cline { 2 - 5 } & Control & 4" N.J. 7150 Ball & N.J. QP2C-M & $\begin{array}{l}\text { NE724 0-20 mA } \\
\text { Electro-Pneumatic }\end{array}$ \\
\hline $\begin{array}{c}\text { Auxiliary } \\
\text { System }\end{array}$ & Shut-Off & $\begin{array}{l}\text { 6" N.J. 815L } \\
\text { Butterfly }\end{array}$ & N.J. QP3C-M & - \\
\cline { 2 - 5 } & Control & $\begin{array}{l}\text { 2" N.J. R21 } \\
\text { Segmented-Ball }\end{array}$ & N.J. QP1C-M & $\begin{array}{l}\text { NE724 0-20 mA } \\
\text { Electro-Pneumatic }\end{array}$ \\
\hline
\end{tabular}

${ }^{1}$ Neles-Jamesbury

\subsubsection{Cascade Test Section}

The test section of the Pratt \& Whitney Canada High-Speed Wind Tunnel contains the cascade and all instrumentation required for data acquisition. The components of the test section are shown in Figure 3.3 .

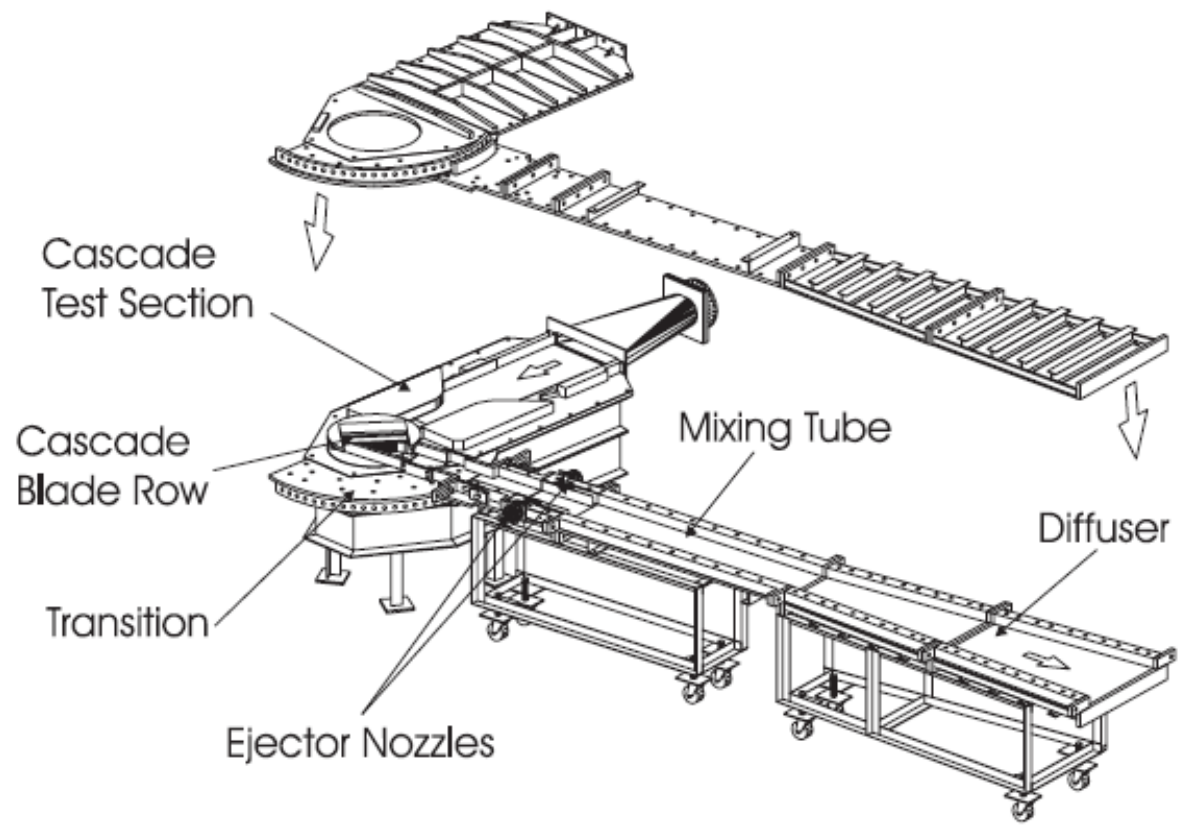

Figure 3.3: Cascade Test Section (Corriveau, 2005). 


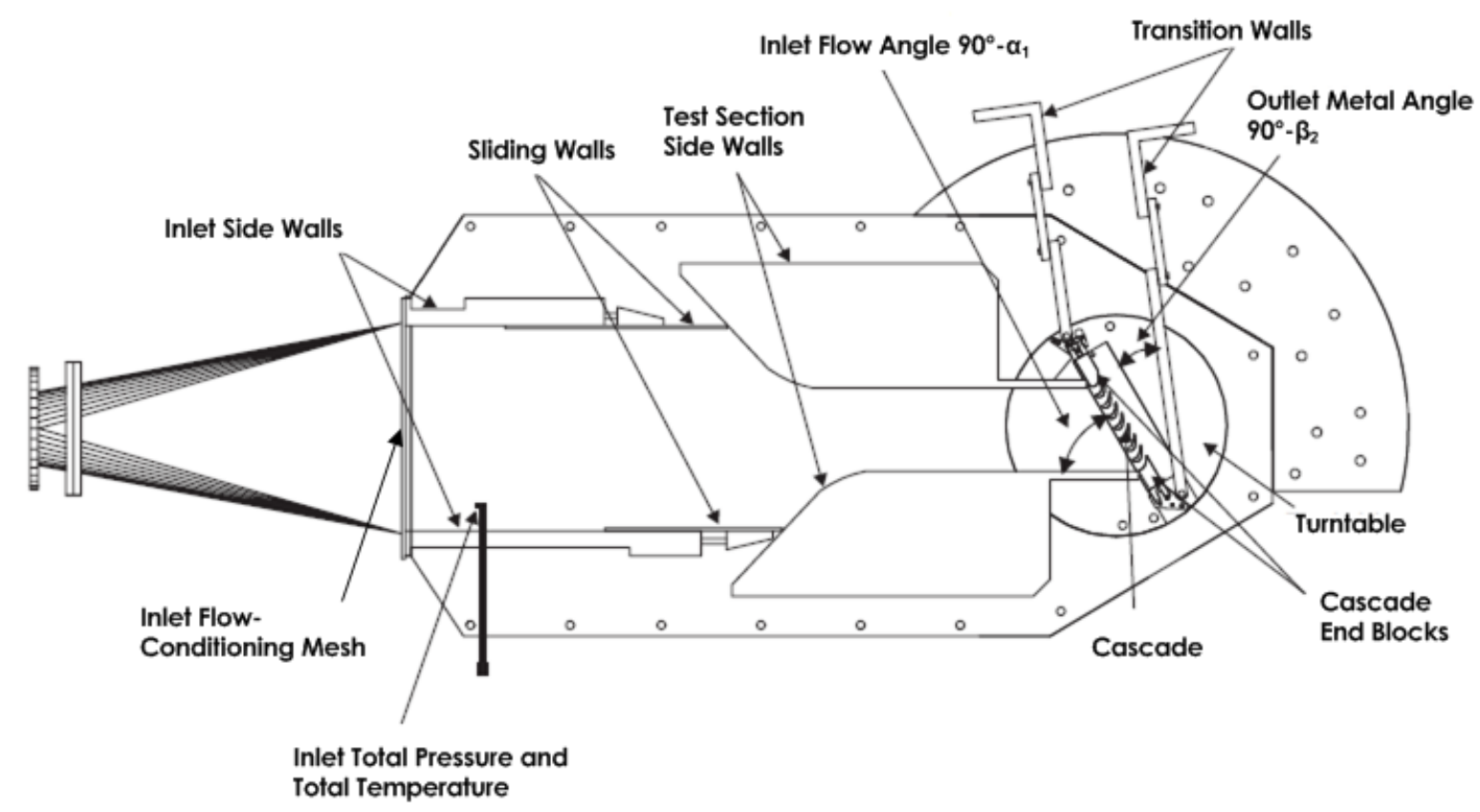

Figure 3.4: Cross-Section of the Inlet Channel and Cascade Assembly (Corriveau, 2005)

The incoming air passes through a circular-to-rectangular transition duct which leads into an inlet channel of rectangular cross section. A detailed layout of the inlet channel is shown in Figure 3.4. A metal flow-conditioning mesh is installed upstream of the inlet channel to promote symmetrical boundary layer growth on the top and bottom endwalls. The mesh wire diameter is 0.018 inch $(0.46 \mathrm{~mm})$, and wires are spaced at 10 wires per inch (roughly 2.5 mm spacing). The inlet boundary layer profile was measured and is described in detail by Corriveau (2005). Typical boundary layer thickness is on the order of $30 \%$ of the blade span, totaling $60 \%$ of the blade span for the top and bottom endwalls. The inlet section features moveable side walls which can be moved in the streamwise and pitchwise directions in order to accommodate cascades of different widths and incidence angles. When correctly installed, the side walls are butted against the two ends of the cascade to form seals. The side walls are placed parallel and aligned with the intended, undisturbed inlet flow direction. The angle of these side walls can be adjusted to fine tune the static pressure distribution at the cascade inlet. The inlet conditions are summarized in Table 3.2 
Table 3.2: Typical Cascade Inlet Conditions (Corriveau, 2005, Jouini, 2000)

\begin{tabular}{ll}
\hline Mach Number & $0.3-0.5$ \\
Turbulence intensity (300 mm upstream of LE) & $\sim 4 \%$ \\
Turbulence length scale $(300 \mathrm{~mm}$ upstream of LE) & $\sim 15 \mathrm{~mm}$ \\
Stagnation temperature $\left(T_{o 1}\right)$ at beginning of run & $\sim 20^{\circ} \mathrm{C}$ \\
Stagnation temperature decrease $\left(\Delta T_{o 1}\right)$ during run & $10^{\circ} \mathrm{C}-20^{\circ} \mathrm{C}$ \\
Endwall boundary layer thickness as percentage of blade span & $\sim 30 \%$ \\
\hline
\end{tabular}

The cascade is mounted on a turntable which can be rotated to vary the incidence angle of the cascade. Using this turntable and a protractor, the incidence angle can be set to within $\pm 1.0^{\circ}$. In the current study, only the design incidence angle of the cascades was examined.

The outlet of the test section is connected to a diffuser duct (also shown in Figure 3.3) which allows some static pressure recovery. Using this diffuser, the static pressure downstream of the cascade is on the order of $70 \mathrm{kPa}(\mathrm{a})$ at an outlet Mach number of 1.2. The air is then exhausted at atmospheric pressure into the laboratory. The wind tunnel facility also has an ejector system. In this system, high pressure air is bled from the storage tanks and passed through two convergent-divergent ejector nozzles, which are placed downstream of the cascade (Figure 3.3). The ejector system thus behaves as a jet pump. It is used to vary the pressure downstream of the cascade in order to vary the Reynolds number (Corriveau, 2005). This capability was not employed in the current study, and the diffuser section was directly attached to the cascade test section.

\subsection{Wind Tunnel Instrumentation}

\subsubsection{Pressure Transducers}

All pressure measurements in the high-speed wind tunnel are performed by eleven Druck PDCR 22 pressure transducers. The roles of these transducers and their measurement ranges are summarized in Table 3.3. Channels 1, 2 and 4, corresponding to the atmospheric, upstream total and downstream static pressures respectively, are required for control of the wind tunnel Mach number. The roles of the remaining channels can be reassigned based on the 
Table 3.3: Druck Pressure Transducer Specifications and Roles

\begin{tabular}{llllll}
\hline Channel & Serial N & Range & Single Port Probe & Three Port Probe & Misc. \\
\hline 1 & 134061 & $100 \mathrm{psi}(\mathrm{d})$ & $P_{\text {atm }}$ & $P_{\text {atm }}$ & $P_{\text {atm }}$ \\
2 & B6228 & $50 \mathrm{psi}(\mathrm{d})$ & $P_{0, r e f}$ & $P_{0, r e f}$ & $P_{0, r e f}$ \\
3 & $82842 / 834$ & $50 \mathrm{psi}(\mathrm{d})$ & $P_{1}$ & $P_{1}$ & Tap 1 \\
4 & 114370 & $50 \mathrm{psi}(\mathrm{d})$ & $P_{2}$ & $P_{2}$ & $P_{2}$ \\
5 & B6992 & $100 \mathrm{psi}(\mathrm{d})$ & Static Port & Port 1 & Tap 2 \\
6 & B3116 & $75 \mathrm{psi}(\mathrm{d})$ & - & Port 2 & Tap 3 \\
7 & 76741 & $100 \mathrm{psi}(\mathrm{a})$ & - & Port 3 & Tap 4 \\
8 & 76740 & $100 \mathrm{psi}(\mathrm{a})$ & - & - & Tap 5 \\
9 & 134060 & $100 \mathrm{psi}(\mathrm{d})$ & - & - & Tap 6 \\
10 & B6202 & $100 \mathrm{psi}(\mathrm{d})$ & - & - & Tap 7 \\
11 & 76738 & $50 \mathrm{psi}(\mathrm{a})$ & - & d: differential a: absolute \\
\hline \multicolumn{5}{r}{}
\end{tabular}

type of measurement being performed. For example, when taking blade surface pressure measurements, channels 3 and 5 through 11 are available to measure static pressure from eight blade surface taps.

The transducers are calibrated using the Druck DPI 605 Pressure Calibrator using a procedure outlined in Appendix B of Hall (2012). The pressure transducers output a voltage signal, and the calibration is used to convert the voltage to a pressure through a linear correlation. The calibration was last performed by Sooriyakumaran (2014), and the curves for the eleven transducers are shown in Figure 3.5 .

\subsubsection{Upstream Reference Total Pressure and Temperature Sensors}

The inlet total pressure and total temperature are measured upstream of the cascade. A cobra probe is used for the total pressure measurement. The probe is placed in the inlet channel as shown in Figure 3.4. Using flexible Tygon tubing, the cobra probe is connected to the Channel 2 transducer indicated in Table 3.3 . The probe is placed well ahead of the cascade such that its wake is dissipated when the flow reaches the cascade. A calibration, described in Appendix A, is applied in order to relate the measured total pressure to a location just 


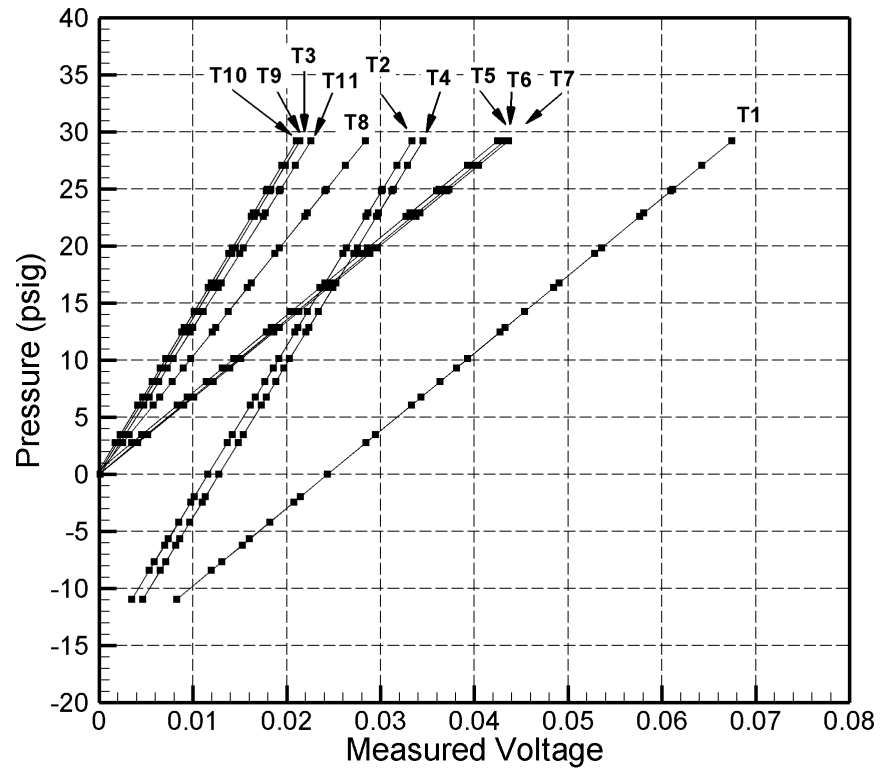

Figure 3.5: Transducer Calibrations Performed by Sooriyakumaran (2014)

ahead of the cascade. The total temperature measurement is accomplished using a K-type thermocouple which is mounted within the cobra probe.

\subsubsection{Pressure Measuring Probes}

\section{Three-Hole Probe}

The total pressure and flow angle are measured using a three-hole probe designed and manufactured by Islam (1999). The dimensions of the three-hole probe are shown in Figure 3.6. The probe is constructed using three stainless steel hypodermic tubes which are soldered together. The central tube is cut squarely at the tip, such that it acts as a pitot tube when the probe is aligned with the flow direction. The two outboard ports have $30^{\circ}$ beveled ends. The flow angle can then be inferred by considering the differential pressure between the two outboard ports.

The probe was calibrated by Sooriyakumaran (2014) and Hall (2012) in a known flow using an in-house calibration rig at Carleton University. The calibration rig employs a convergent nozzle, which limits the available Mach number to the subsonic range. In order to take 


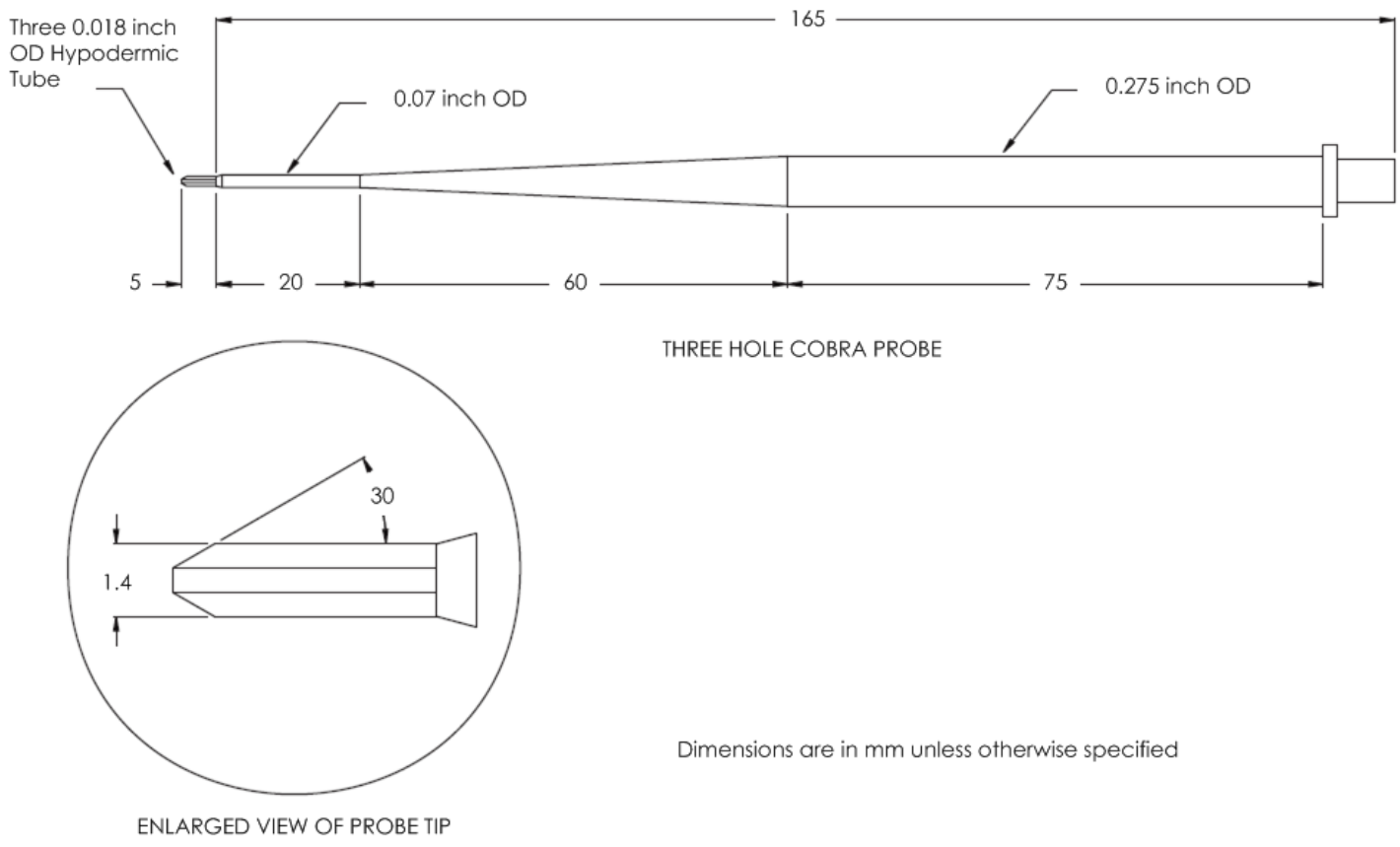

Figure 3.6: Three-Hole Probe Manufactured by Islam (1999)

measurements in supersonic flow, a Rayleigh correction is applied to the probe's total pressure measurements to account for the standing normal shock wave at the tip of the probe, as will be described in Section 4.4.4. Correct application of the Rayleigh correction requires knowledge of the true static pressure. The static pressure measured by the three-hole probe cannot be used since it is affected by the shock wave at the probe tip. For this reason, the true static pressure is measured separately with a static probe.

\section{Static Probe}

The static pressure probe is used to measure the static pressure directly. The static probe dimensions are shown in Figure 3.7. It was designed by Islam (1999) with a $15^{\circ}$ conical tip to minimize the effect of the probe tip shock wave that occurs in supersonic flow. Static pressure is measured through two holes drilled on opposite sides of the probe's hollow tube. Since the largest pressure gradients in the cascade flow occur in the pitchwise direction, the two holes are aligned vertically. 

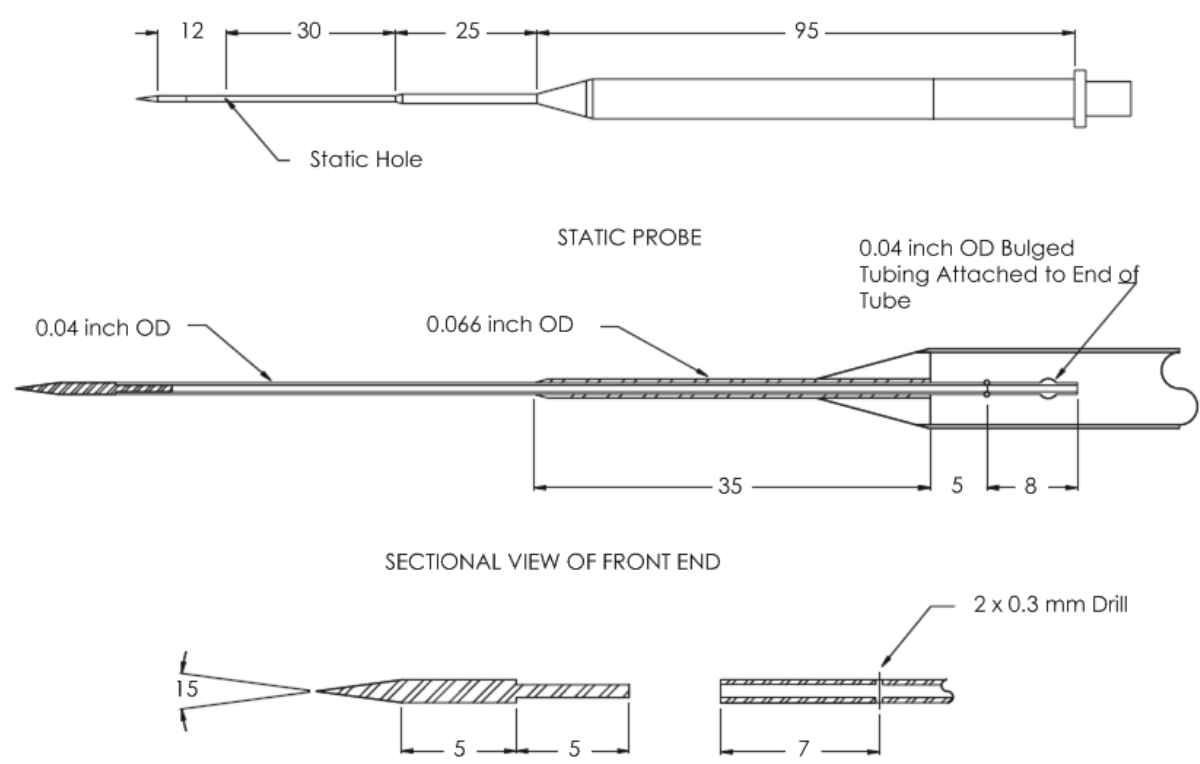

ENLARGED ASSEMBLY OF PROBE TIP

Dimensions are in mm unless otherwise specified

Figure 3.7: Static Probe Manufactured by Islam (1999)

\subsubsection{Probe Traverse Mechanism}

The pressure measuring probes are traversed across the wakes downstream of the cascade using the probe traverse mechanism shown in Figure 3.8. The probes cannot be used simultaneously, but can be mounted interchangeably on a probe stem which moves across the test section at a fixed distance downstream of the cascade. The arm has sharp leading and trailing edges and a narrow profile to reduce the effect of drag and blockage on the pressure downstream of the cascade. The arm position is controlled by a stepper motor connected to a threaded rod. This mechanism is used to incrementally traverse the probe between measurement points during a run. Details about the spacing between these measurement points are given in Section 4.3.1. 


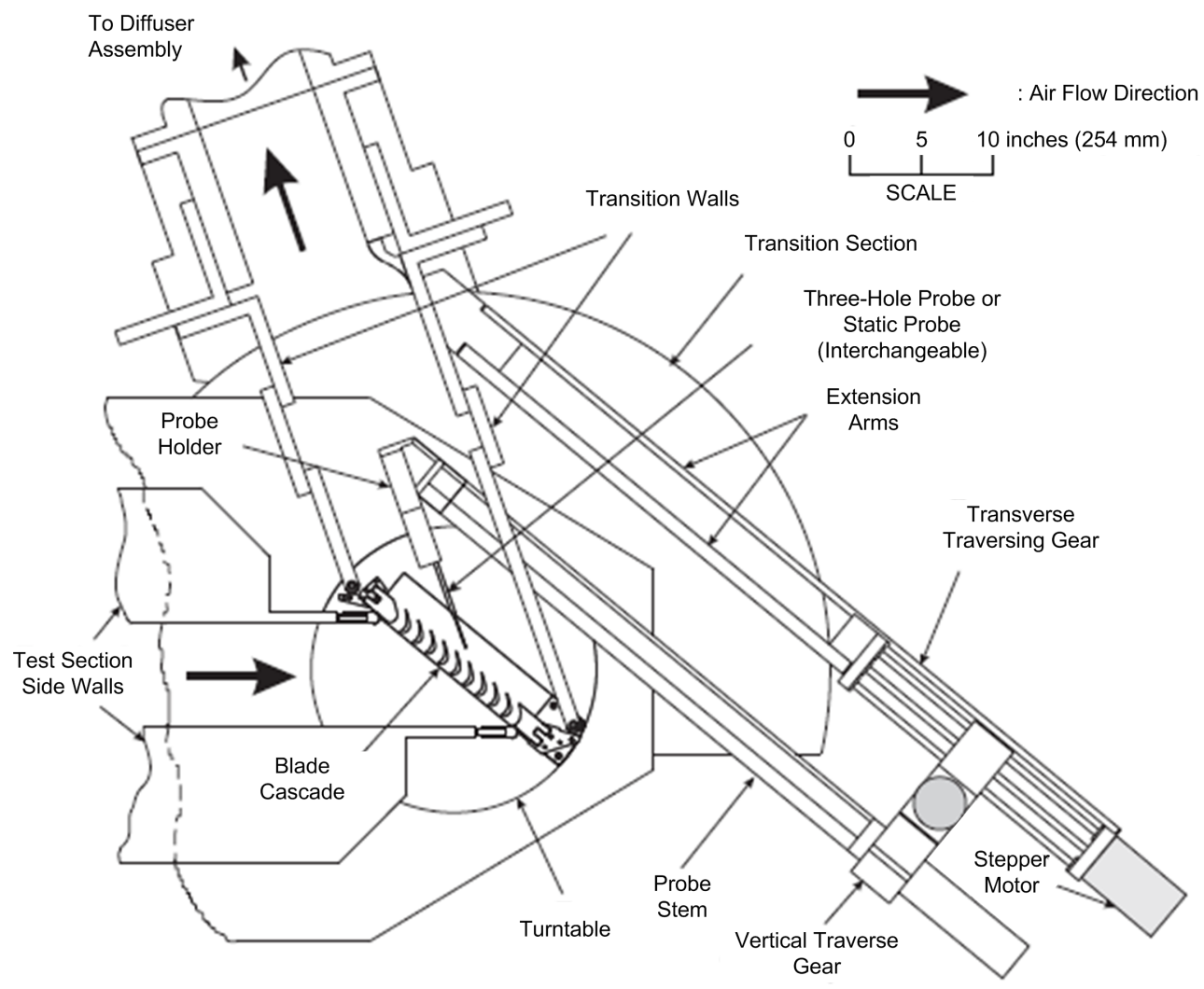

Figure 3.8: Downstream Probe Traversing Mechanism (Adapted from Corriveau, 2005) 


\subsection{Cascade Geometry}

\subsubsection{AF4 Turbine Cascade}

The cascade of turbine blades used for testing in the wind tunnel is designated AF4. Prior to the present investigation, four cascades were supplied to Carleton University by Siemens Energy Inc., designated AF1 to AF4. Although the same axial chord, pitch, span and trailing edge thickness were used for all cascades, each cascade featured a different loading distribution. AF1 served as a mid-loaded reference cascade. AF4 was the most front-loaded cascade and had low suction side curvature downstream of the throat (low unguided turning). The objective of the study was to investigate the effect of the loading distribution on the transonic profile losses. Based on this study, all blades exhibited the loss plateau described in Section 2.5.1. However, the final rise in losses at high Mach numbers was not observed in AF4, suggesting that the loss plateau for AF4 might extend to higher Mach numbers than for the other cascades. This behaviour, combined with the low unguided turning angle $\left(\theta_{u}\right)$, makes the AF4 cascade an interesting case for further investigation.

Profile loss measurements were made for AF4 prior to the current study, but only five data points were taken at different Mach numbers. In the current study, measurements were made at sixteen Mach numbers over a Mach number range of about 0.5 to 1.4 in order to better understand the flow behavior in the transonic and supersonic regimes. In addition to experimental wind tunnel testing, numerical simulations were performed at matching conditions, as described in Chapter 5 .

Figure 3.9 summarizes the symbols used to define cascade geometry. The geometric parameters for the AF4 cascade are provided in Table 3.4. The airfoil shape of the AF4 cascade is shown in Figure 3.10, and a plot of the surface curvature is provided in Figure 3.11. The surface curvature is expressed as $C_{x} / R$, which is the ratio of the axial chord $\left(C_{x}\right)$ to the local radius of curvature $(\mathrm{R})$.

In addition to loss data for AF4 measured in the present study, previously measured data for three other cascades (HS1A, HS1C and HS1D) were used. The geometry of these cascades are also included in Table 3.4 and the cascades are described further in the next section. 


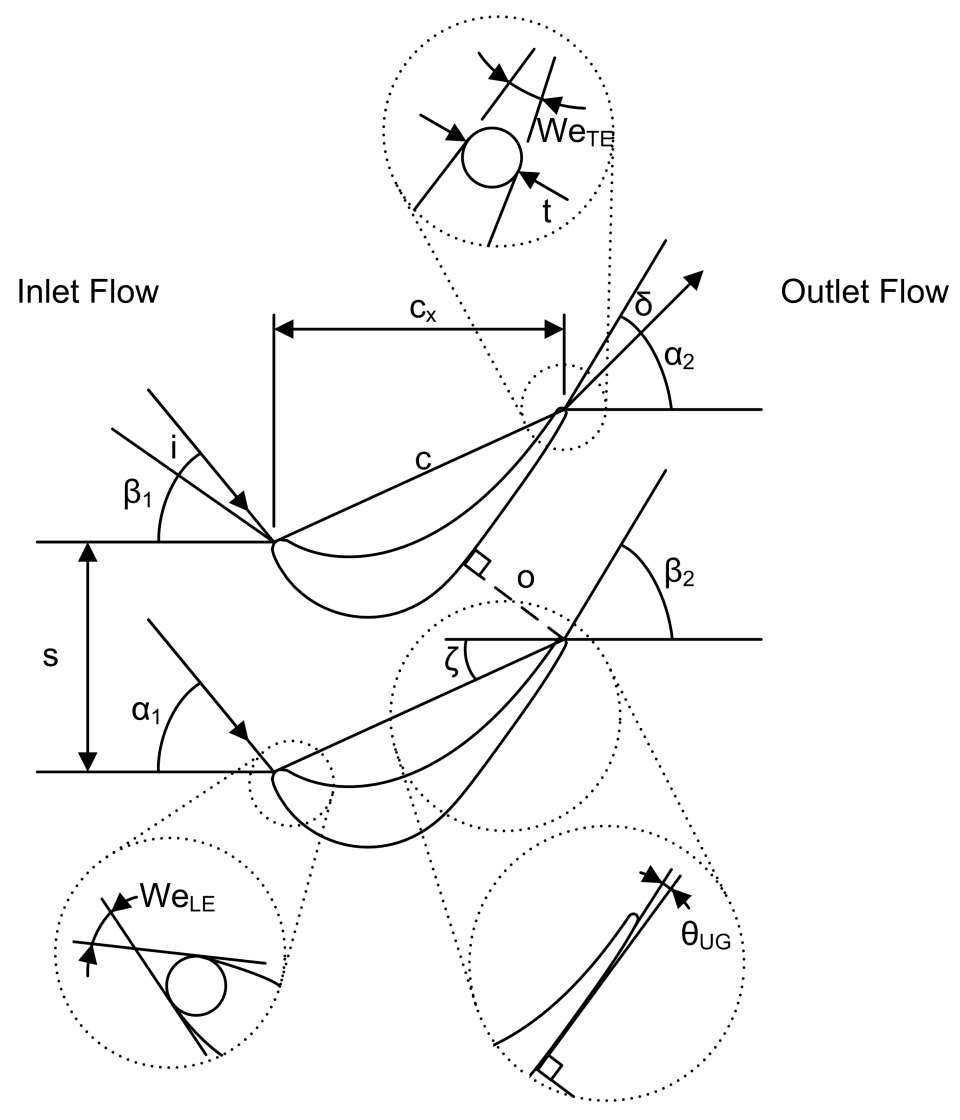

Figure 3.9: Summary of Symbols Used to Define Cascade Geometry (Adapted from Corriveau, 2005)
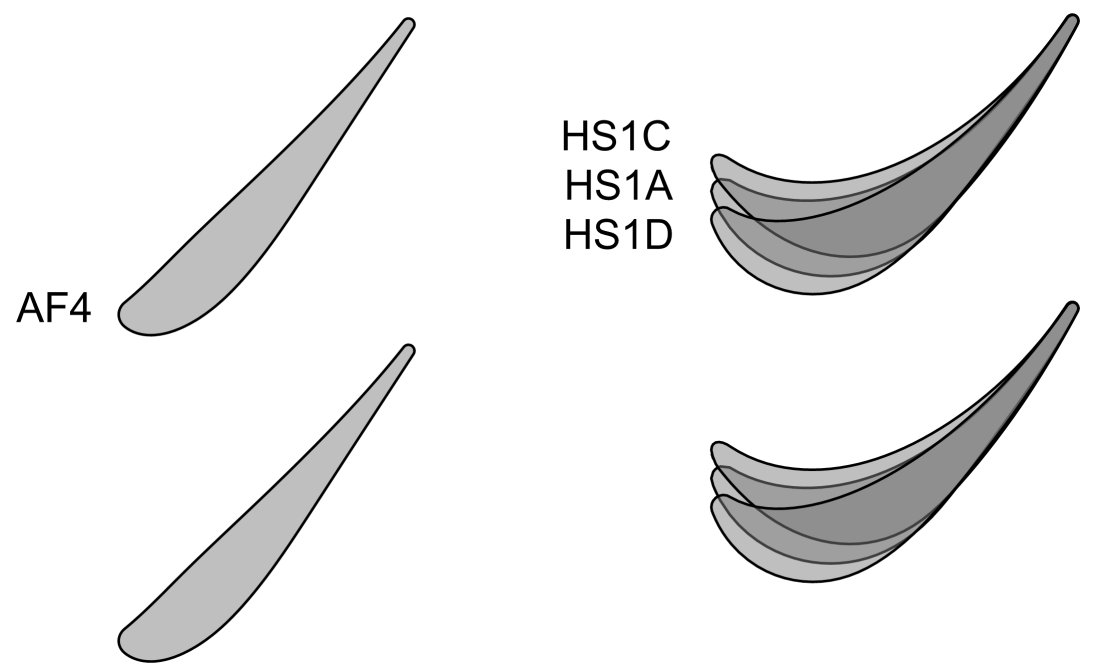

Figure 3.10: Profiles of the AF4 and HS1 Family of Cascades (Actual Size) 
Table 3.4: Cascade Geometric Parameters

\begin{tabular}{|c|c|c|c|c|c|}
\hline Symbol & Parameter & $\mathrm{AF} 4$ & HS1A & HS1C & HS1D \\
\hline$C$ & Chord Length & $42.22 \mathrm{~mm}$ & $41.2 \mathrm{~mm}$ & $40.4 \mathrm{~mm}$ & $43.3 \mathrm{~mm}$ \\
\hline$C_{x}$ & Axial Chord & $30.04 \mathrm{~mm}$ & $37.3 \mathrm{~mm}$ & $37.3 \mathrm{~mm}$ & $37.3 \mathrm{~mm}$ \\
\hline$h$ & Blade Span & $61 \mathrm{~mm}$ & $61 \mathrm{~mm}$ & $61 \mathrm{~mm}$ & $61 \mathrm{~mm}$ \\
\hline$s$ & Blade pitch & $33.105 \mathrm{~mm}$ & $29.14 \mathrm{~mm}$ & $29.14 \mathrm{~mm}$ & $29.14 \mathrm{~mm}$ \\
\hline$t_{\max }$ & Maximum Thickness & $6.68 \mathrm{~mm}$ & $7.9 \mathrm{~mm}$ & $8.0 \mathrm{~mm}$ & $7.9 \mathrm{~mm}$ \\
\hline$h / C$ & Aspect Ratio & 1.445 & 1.481 & 1.51 & 1.409 \\
\hline$s / C$ & Solidity & 0.784 & 0.707 & 0.721 & 0.673 \\
\hline$\zeta$ & Stagger Angle & $45.13^{\circ}$ & $25.1^{\circ}$ & $22.6^{\circ}$ & $30.5^{\circ}$ \\
\hline$\beta_{1}$ & Inlet Metal Angle & $0.28^{\circ}$ & $50.5^{\circ}$ & $50.5^{\circ}$ & $50.5^{\circ}$ \\
\hline$\alpha_{1}$ & Inlet Flow Angle & - & $46^{\circ}$ & $46^{\circ}$ & $46^{\circ}$ \\
\hline$i_{\text {des }}$ & Design Incidence & - & $-4.5^{\circ}$ & $-4.5^{\circ}$ & $-4.5^{\circ}$ \\
\hline$W e_{L E}$ & LE Wedge Angle & $77.91^{\circ}$ & $38^{\circ}$ & $15^{\circ}$ & $38^{\circ}$ \\
\hline$a / b$ & LE Ellipse Ratio & 1.0625 & 1 & 2.1 & 1 \\
\hline$b$ & LE Minor Axis & $1.6 \mathrm{~mm}$ & $1.02 \mathrm{~mm}$ & $1.02 \mathrm{~mm}$ & $1.02 \mathrm{~mm}$ \\
\hline$\beta_{2}$ & Outlet Metal Angle & $54.39^{\circ}$ & $59^{\circ}$ & $59^{\circ}$ & $59^{\circ}$ \\
\hline$\alpha_{2}$ & Outlet Flow Angle & $54.67^{\circ}$ & $58.5^{\circ}$ & $58.5^{\circ}$ & $58.5^{\circ}$ \\
\hline$t$ & TE Thickness & $1.22 \mathrm{~mm}$ & $1.26 \mathrm{~mm}$ & $1.26 \mathrm{~mm}$ & $1.26 \mathrm{~mm}$ \\
\hline$o$ & Throat Opening & $16.93 \mathrm{~mm}$ & $15.9 \mathrm{~mm}$ & $15.9 \mathrm{~mm}$ & $15.9 \mathrm{~mm}$ \\
\hline$t / o$ & TE to Throat Ratio & 0.072 & 0.079 & 0.079 & 0.079 \\
\hline$W e_{T E}$ & TE Wedge Angle & $5^{\circ}$ & $6^{\circ}$ & $6^{\circ}$ & $6^{\circ}$ \\
\hline$\theta_{u g}$ & Unguided Turning & $3.68^{\circ}$ & $11.5^{\circ}$ & $14.5^{\circ}$ & $11.5^{\circ}$ \\
\hline$\Phi$ & $\begin{array}{l}\text { Sieverding et al. (1979) } \mathrm{Pa} \text { - } \\
\text { rameter }\end{array}$ & $4.34^{\circ}$ & $8.75^{\circ}$ & $10.25^{\circ}$ & $8.75^{\circ}$ \\
\hline$M_{1}$ & Inlet Mach Number & - & 0.53 & 0.53 & 0.53 \\
\hline$M_{2}$ & Outlet Mach Number & - & 1.04 & 1.04 & 1.04 \\
\hline$Z$ & Zweifel Coefficient & - & 0.856 & 0.856 & 0.856 \\
\hline
\end{tabular}




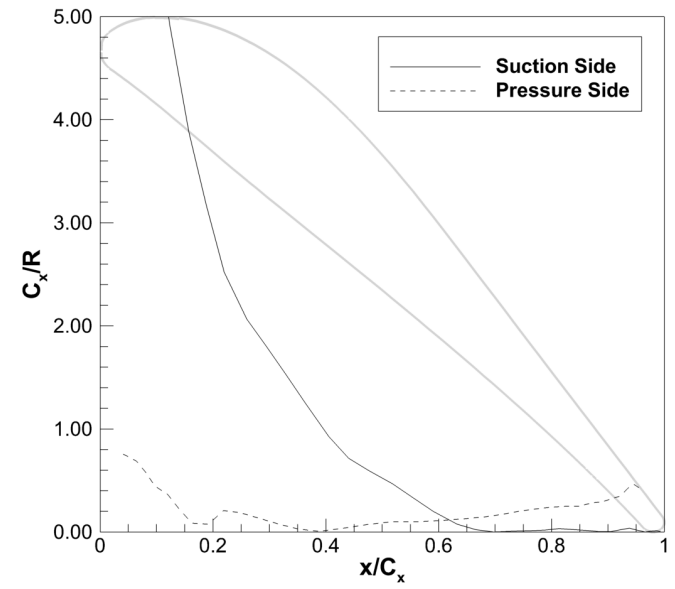

(a) $\mathrm{AF} 4$

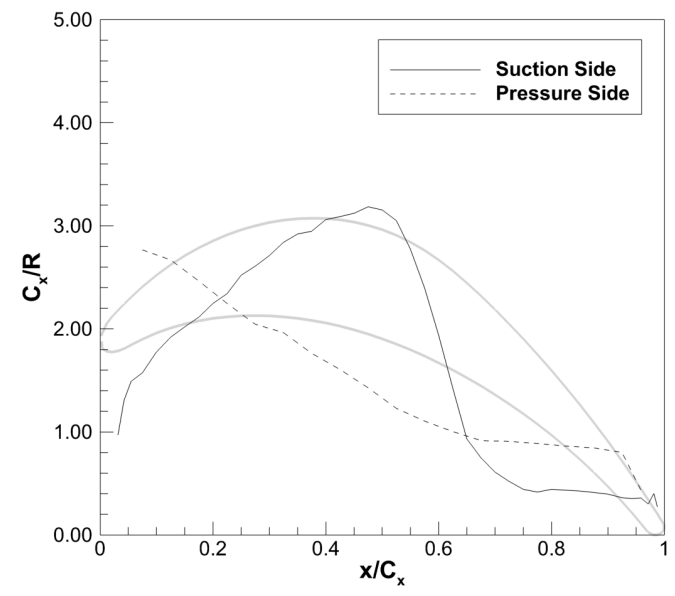

(c) HS1C

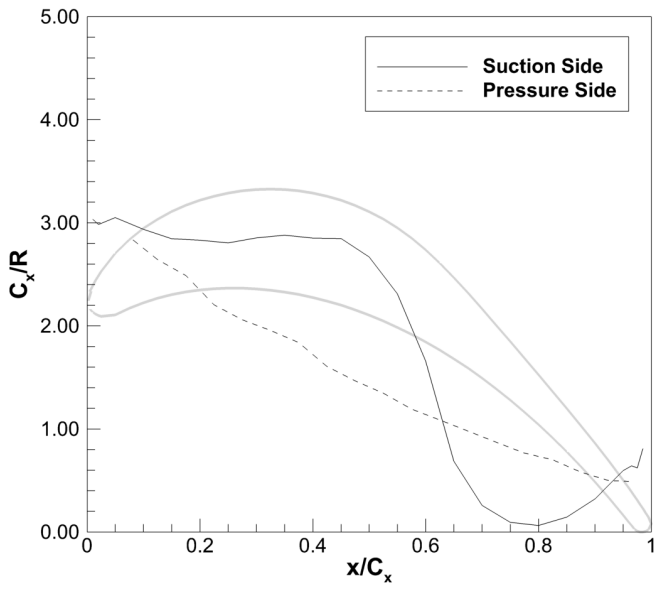

(b) HS1A

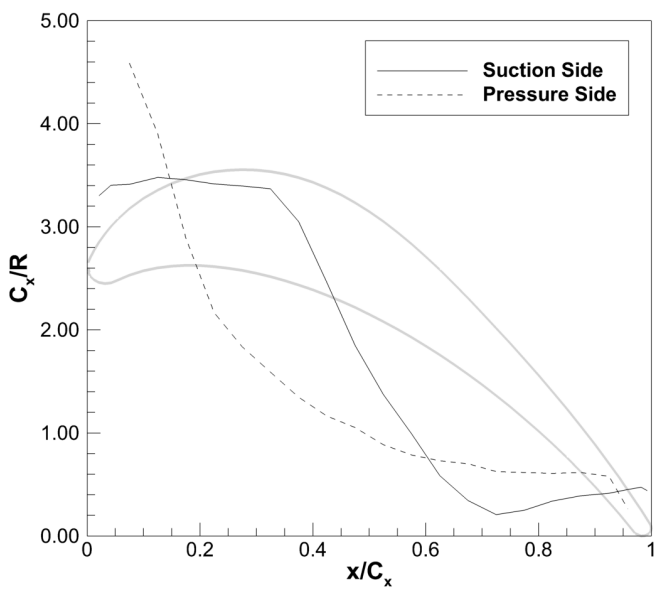

(d) HS1D

Figure 3.11: Surface Curvature of Airfoils, with Airfoil Shape Shown in Background 


\subsubsection{HS1 Turbine Cascade Family}

The HS1 family of cascades was the subject of an investigation on transonic profile losses by Corriveau (2005). The HS1A cascade served as a baseline, and was derived from the midspan profile of a turbine blade used in an engine by Pratt \& Whitney Canada. The HS1C and HS1D cascades were designed by Corriveau using Pratt \& Whitney Canada in-house blade design tools. The loading distributions were modified to create more front-loaded and aft-loaded blades compared to the mid-loaded HS1A. In order to maintain the same velocity triangles, the loading distributions were mainly modified by changing the stagger angles. The same axial chord, pitch, span and trailing edge thickness were maintained across all three airfoils. Corriveau used the cascades to investigate the effect of blade loading distribution on the total pressure losses in the transonic regime. He identified a loss plateau that occurred above Mach 1.0, and attributed the behavior to the variations in base pressure. However, the limited data at different Mach numbers made it difficult to confidently understand the loss trends.

In the current study, the HS1 cascade midspan flows are numerically simulated at a much finer resolution of Mach numbers than the measurements and simulations performed by Corriveau. The numerical simulations in the current study are compared to Corriveau's experimental measurements.

As mentioned in the previous section, the geometric parameters of the HS1 cascades are included in Table 3.4. The airfoil shape and surface curvature of the HS1 cascades are shown in Figures 3.10 and 3.11 together with the AF4 cascade.

The experimental apparatus used in this investigation has now been fully described. The next chapter explains how this apparatus is used. The data collection and reduction methods employed to obtain experimental data are described, and an estimate of the measurement uncertainty is provided. 


\section{Chapter 4}

\section{Experimental Procedures and Measurement Uncertainty}

\subsection{Introduction}

The experimental procedures employed in the high-speed wind tunnel are discussed in the current chapter. The Mach number control system of the wind tunnel is described first, followed by the data acquisition methods. The data processing methods are explained, including the required calibrations and corrections. The uncertainty in the measurements is then quantified.

\subsection{Control System}

When the high-speed wind tunnel was first commissioned by Jeffries (1994), the operating point was controlled based on holding the blowing pressure (or upstream total pressure $P_{o 1}$ ) at a specified value. A Proportional-Integral (P.I.) control system was used to position the control valve which regulates the blowing pressure. This arrangement was problematic because the Mach number in the test section is a function of both the upstream total pressure $\left(P_{o 1}\right)$ and the downstream static pressure $\left(P_{2}\right)$. Since the wind tunnel air is exhausted into the laboratory, daily variations in atmospheric pressure caused variations in this pressure ratio. Therefore, for a given blowing pressure, the Mach number in the test section could vary on different testing days. 
The high-speed wind tunnel control system was recently redesigned by Hall (2012). The wind tunnel is now controlled on the basis of the Mach number directly, by sampling both the upstream total pressure $\left(P_{o 1}\right)$ and the downstream static pressure $\left(P_{2}\right)$. The following isentropic Mach number relation is employed:

$$
\frac{P}{P_{o}}=\left(1+\frac{\gamma-1}{2} M^{2}\right)^{\frac{-\gamma}{\gamma-1}}
$$

where $\gamma$ is the ratio of specific heats. The upstream total pressure measurement is taken from the pitot tube in the inlet duct, corresponding to Channel 2 in Table 3.3. The downstream static pressure (Channel 4, Table 3.3) is obtained from a small manifold connected to four of the downstream endwall static taps, effectively averaging the pressure across the taps. This manifold is used because the static pressure read by any single endwall static tap can vary greatly at different operating points due to the complex shock and wake patterns that arise downstream of the blade trailing edges.

The control system uses a feedback loop, as shown in Figure 4.1. In the box labeled "Sensor", the two pressure measurements are taken by the pressure transducers and transmitted as voltage signals. A 16-bit National Instruments (NI) PCI-6229A data acquisition card is used to acquire the voltage signals. The card is connected to a Microsoft Windows-based PC. The control system software, written in LabVIEW8, receives the voltage signals and converts them to pressures using calibration curves generated by Sooriyakumaran (2014) shown in Figure 3.5. These calibrations are linear functions of the measured voltage $(V)$ :

$$
P=m V+b
$$

The slope $(m)$ stays nearly constant, but the offset $(b)$ tends to drift as a result of changing atmospheric pressure in the laboratory. For this reason, the offset value is measured at the beginning of every test day as part of the tunnel start-up procedures. Once the pressure measurements are obtained, the isentropic Mach number in the tunnel is calculated using 


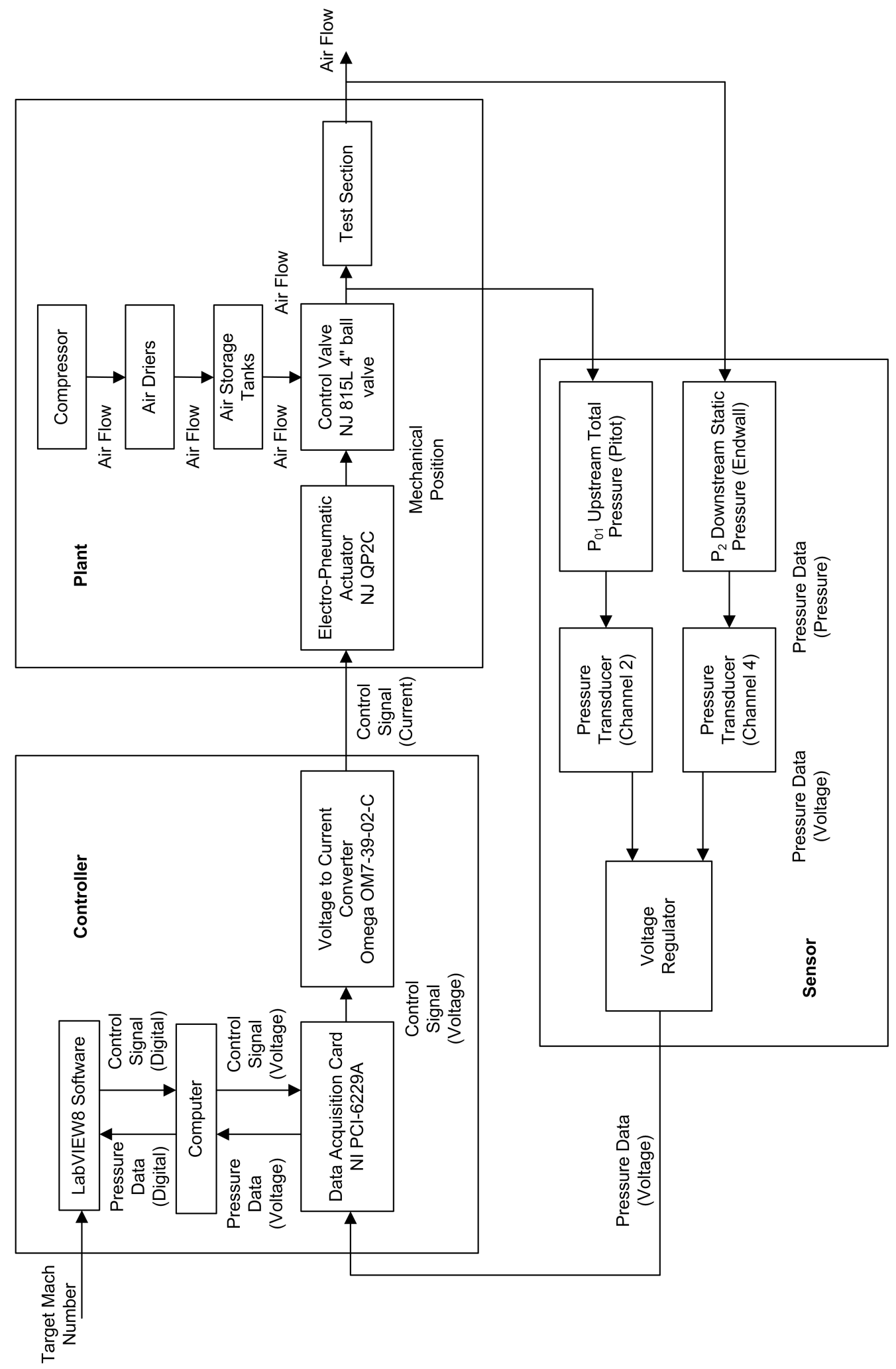

Figure 4.1: Flow Diagram of the Wind Tunnel Mach Number Control System (Adapted from Hall, 2012) 
Equation 4.1. A P.I.D. control system, designed by Hall (2012), is used control the valve position.

The NI PCI-6229A data acquisition card then provides a voltage output for control valve positioning. The wind tunnel control valve is positioned using an electro-pneumatic actuator (Neles-Jamesbury model QP2C), which is controlled on the basis of current. For this reason, the voltage signal from the data acquisition card is converted to current (0-20 mA) using an Omega OM7-39-02-C voltage to current converter. The tunnel blowing pressure is then controlled using a $10.2 \mathrm{~cm}$ (4 in) Neles-Jamesbury model 815L butterfly valve.

The isentropic Mach number calculated by the control system assumes that the upstream total pressure $\left(P_{o 1}\right)$ is constant across the cascade. However, total pressure losses across the cascade lead to a lower downstream total pressure $\left(P_{o 2}\right)$. Therefore, the true Mach number in the test section is lower than the isentropic Mach number, and is dependent on the total pressure losses through the cascade. The actual downstream Mach number is calculated from pressure probe data as part of the final data reduction procedures.

\subsection{Data Acquisition Procedures}

\subsubsection{Measurement Locations}

The locations of the measurements made in the cascade test section are shown in Figure 4.2. As described in Section 4.2, the control system operates based on two pressure measurements: the upstream total pressure and the downstream static pressure. The upstream total pressure is measured using a pitot tube far upstream of the cascade, so as not to disturb the flow into the cascade. In order to account for losses in the inlet duct, the total pressure measurement is corrected to a distance of roughly $60 \mathrm{~mm}$ upstream of the cascade leading edge, according to the method which is described in Appendix A.

Two rows of $0.4 \mathrm{~mm}$ diameter static pressure taps are drilled into the Lexan window that forms the top endwall and seals the test section. The rows are placed across the cascade inlet and outlet, allowing the static pressure distribution across all blades to be measured. The inlet row is positioned 1.0 axial chord lengths $\left(C_{x}\right)$ upstream of the cascade leading edge and 


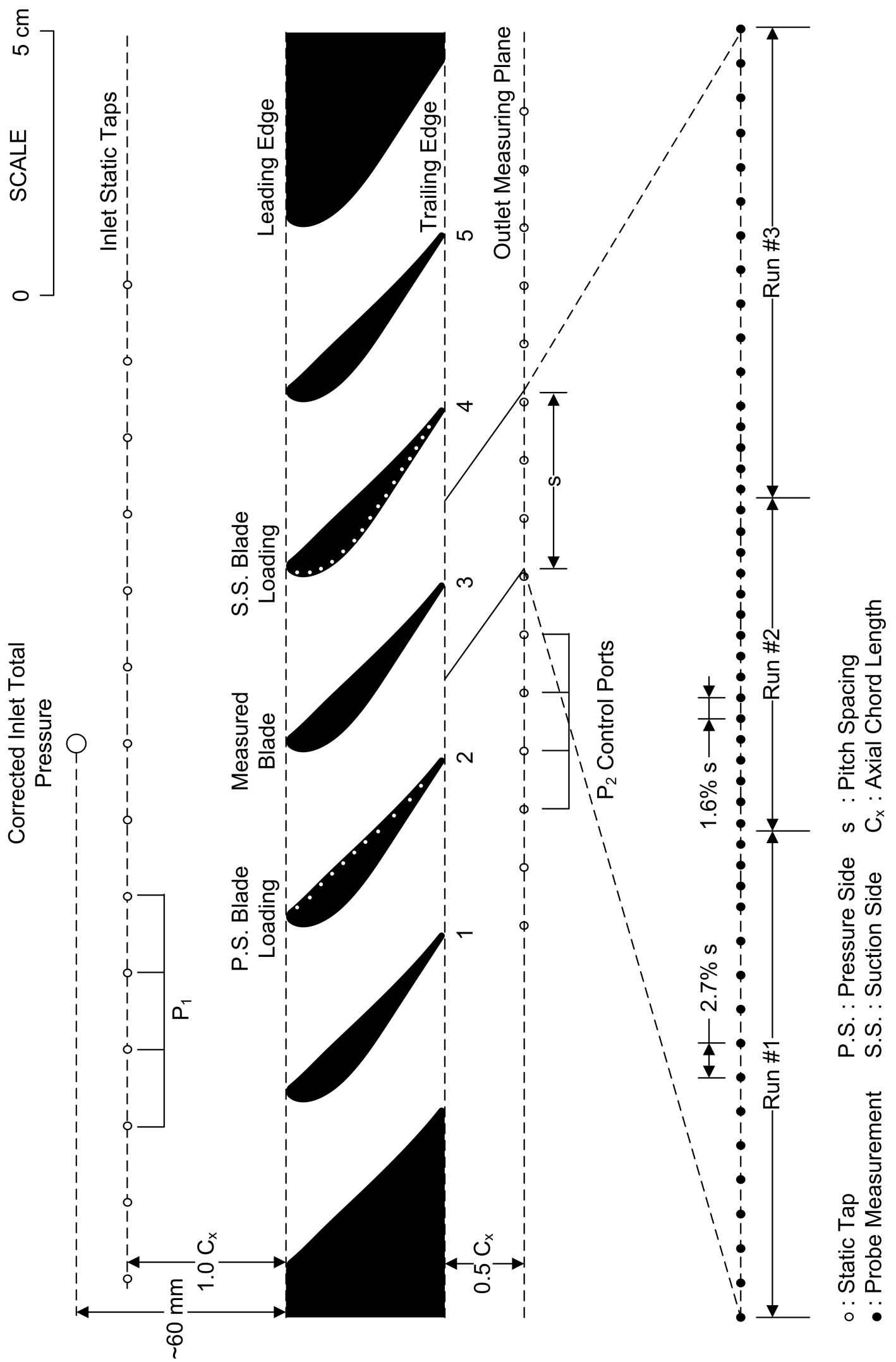

Figure 4.2: Measurement Locations in the Cascade Test Section 
contains fourteen taps. The outlet row is positioned 0.5 axial chord lengths $\left(C_{x}\right)$ downstream of the cascade trailing edge and contains fifteen taps. Prior to testing, all static pressures are measured to ensure that the upstream static pressure distribution is uniform and that the downstream static pressure distribution is periodic.

For the wind tunnel control system, the outlet static pressure $\left(P_{2}\right)$ is measured by connecting a manifold to four of the outlet static pressure taps at the location indicated in Figure 4.2 . While probe measurements are taken, the inlet static pressure $\left(P_{1}\right)$ is also collected using a manifold connected to the four inlet static pressure taps indicated.

The blade loading data is collected via static ports built into blades 2 and 4 . Blade 2 contains 11 static ports on the pressure side, while blade 4 contains 16 static ports on the suction side. The blade used for the loss measurements (blade 3) was deliberately left without instrumentation in order to avoid disturbing the blade surface flow, and thus affecting wake measurements.

Wake data in the pitchwise direction is collected using the three-hole probe and static probe at 0.5 axial chord lengths downstream of the cascade trailing edge. Data is collected at a total of 48 discrete locations across one blade pitch $(s)$, downstream of the middle blade. Due to the limited mass of pressurized air in the storage tanks, wind tunnel run times are on the order of 40 seconds, and are further reduced at higher Mach numbers. The 48 data points are measured over three runs of sixteen data points each, in order to ensure that data is only collected while the wind tunnel can maintain steady state operation.

\subsubsection{Data Acquisition System}

The data acquisition system was most recently updated by Taremi et al. (2008), and is built into the same LabVIEW8 software as the control system. As with the control system, all pressures are detected by the pressure transducers described in Table 3.3. The resulting voltage signals are amplified and recorded by the 16-bit National Instruments (NI) PCI-6229A data acquisition card. The upstream total temperature reading from the K-type thermocouple is amplified, filtered and recorded using a 24-bit NI USB-9211A unit. 


\subsubsection{Sampling Rates}

Due to the inherent unsteadiness of an intermittent supersonic blow-down wind tunnel, it is necessary to ensure that adequate sampling rates and times are chosen to represent the desired steady-state condition. To this end, the dynamic behaviour of the wind tunnel was investigated by Jeffries et al. (1997). The fluctuations observed in the tunnel can be divided into low frequency and high frequency ranges.

Across a large range of Mach numbers, Jeffries et al. (1997) detected a low frequency (about $0.5 \mathrm{~Hz}$ ) fluctuation in all pressure measurements, with a magnitude of about $1 \%$ of the mean measured value. All readings were in phase, so the fluctuation was attributed to the wind tunnel control system. As a correction method, all pressures are normalized by the simultaneously measured upstream total pressure. Despite removing the low frequency fluctuations in all pressure measurements, this method does not correct for the effect of changing Mach number, which affects the losses, and by extension the pressures measured downstream. The losses are particularly sensitive to Mach number fluctuations in the transonic Mach number range, where shocks rapidly appear with small changes in Mach number. Furthermore, the effect of wind tunnel dynamics on pressure measurements has not been assessed since the new control system was commissioned by Hall (2012). However, based on Hall's assessment of the new control system, the magnitude of low frequency fluctuations is reduced and Mach number control is improved.

Regarding the wind tunnel's high frequency behavior, Jeffries et al. (1997) observed pressure fluctuations up to $1000 \mathrm{~Hz}$. According to the Nyquist criterion, measurements must be taken at twice the highest perceived frequency in order to obtain representative data. For this reason, data is recorded at $2000 \mathrm{~Hz}$. Jeffries et al. (1997) also investigated the period of time over which data should be sampled and averaged. It was determined that averaging measurements over 0.1 second is sufficient to obtain a pressure measurement with less than $1 \%$ uncertainty in the averaged pressure value. Thus, for every data point obtained, pressure is measured at $2000 \mathrm{~Hz}$ for 0.1 second and averaged, totaling 200 measurements per data point. 


\subsection{Data Reduction Procedures}

\subsubsection{Introduction}

Once measurements have been made, some manipulations are required to obtain useful information from the raw data. The following section describes these mathematical manipulations. Firstly, the procedures for obtaining blade loading are provided. Secondly, the process for converting probe pressure measurements into wake flow data is presented. The process begins by applying appropriate probe calibration, followed by a correction for the shock wave at the tip of the three-hole probe. The static pressure distribution from the static probe must be aligned with that of the three-hole probe. The wake data must then be "mixed-out" to uniform conditions to account for all mixing losses that occur downstream of the measuring plane.

\subsubsection{Blade Loading Measurements}

Blade loading data are collected over the same range of Mach numbers as the downstream traverses. The blade surface static pressures $\left(\left(P_{s}\right)_{x}\right)$ are obtained using the static ports in blades 2 and 4 . The total pressure just upstream of the cascade $\left(P_{o 1}\right)$ is obtained by taking the reference total pressure measurement $\left(P_{o, r e f}\right)$ far upstream of the cascade and applying the correction described in Appendix $\mathrm{A}$ to account for losses in the inlet duct. The isentropic Mach number on the blade surfaces is then calculated using the following isentropic Mach number relation:

$$
\left(M_{s}\right)_{x}^{2}=\frac{2}{\gamma-1}\left[\left(\frac{P_{o 1}}{\left(P_{s}\right)_{x}}\right)^{\frac{\gamma-1}{\gamma}}-1\right]
$$

\subsubsection{Three-Hole Probe and Static Probe Data Reduction}

Prior to being used to record data, the three-hole probe's output must be calibrated. The three-hole probe was most recently calibrated by Sooriyakumaran (2014) for high subsonic Mach numbers, and by Hall (2012) for mid to low subsonic Mach numbers. The calibration procedures are described in detail by Sooriyakumaran (2014). 


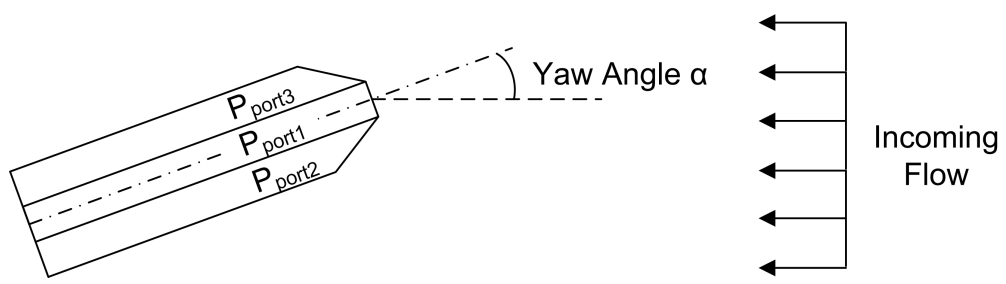

Figure 4.3: Schematic of the Three-Hole Probe Tip, Showing the Locations of the Three Pressure Ports

The probe calibration consists of the following calibration coefficients, given for a range of Mach numbers and yaw angles $(\alpha)$. The coefficients are defined based on the three port pressures $\left(P_{\text {port } 1}, P_{\text {port } 2}\right.$ and $\left.P_{\text {port } 3}\right)$, shown schematically in Figure 4.3 , as follows:

$$
\begin{aligned}
\text { Yaw angle coefficient } & C_{\alpha}=\frac{P_{\text {port } 2}-P_{\text {port } 3}}{P_{\text {port } 1}-\bar{P}} \\
\text { Total pressure coefficient } & C_{o}=\frac{P_{o}-P_{\text {port } 1}}{P_{\text {port } 1}-\bar{P}} \\
\text { Static pressure coefficient } & C_{s}=\frac{P_{o}-P_{s}}{P_{\text {port } 1}-\bar{P}}
\end{aligned}
$$

where

$$
\bar{P}=\frac{P_{\text {port } 2}+P_{\text {port } 3}}{2}
$$

Calibrations were performed at Mach numbers ranging from 0.2 to 0.98 . At intermediate Mach numbers, new calibrations can be generated by linearly interpolating between two original calibrations. The calibration rig has a converging nozzle without a diverging section. As a result, only subsonic calibrations can be obtained. When probe measurements are made at supersonic Mach numbers, the highest calibration is used (Mach 0.98) and a Rayleigh shock correction is applied to account for the normal shock ahead of the probe, as will be described in Section 4.4.4. The calibration curves obtained by Sooriyakumaran are provided in Figure 4.4 .

The raw probe data from the wind tunnel has the form of port pressures. The data reduction procedures are used to obtain the flow angle, total pressure and static pressure. The procedures begin by dividing all probe pressures by the upstream total pressure $\left(P_{o 1}\right)$. Guessing a Mach number allows new calibration curves to be generated by linearly interpolating 


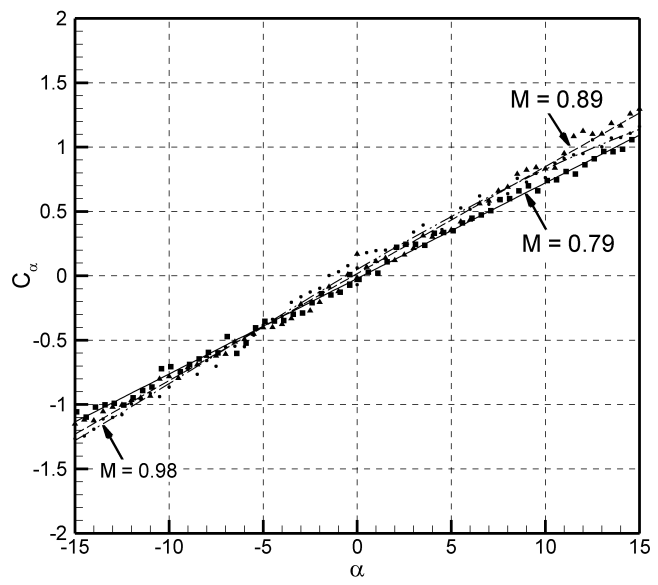

(a) $C_{\alpha}$ Calibration Curve

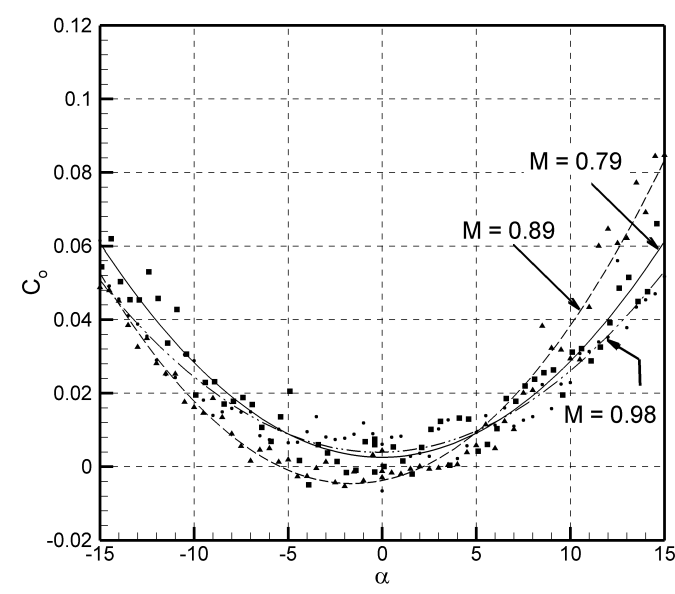

(b) $C_{0}$ Calibration Curve

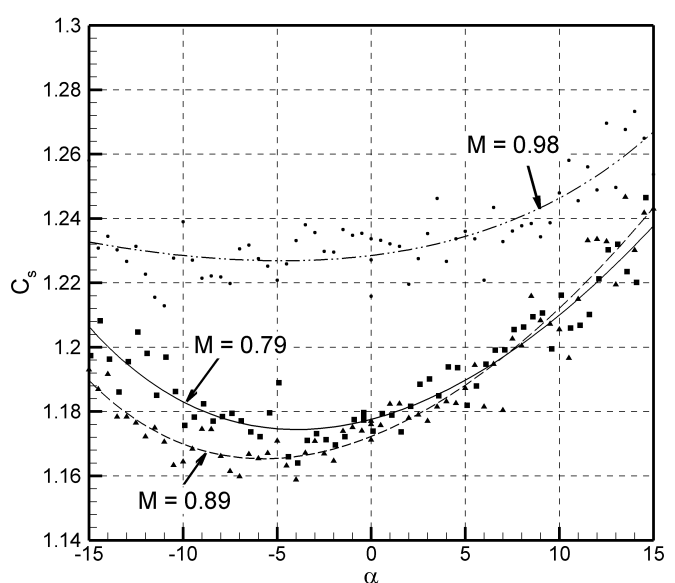

(c) $C_{s}$ Calibration Curve

Figure 4.4: Three-Hole Probe Calibration Curves Used in the Current Study, Collected by Sooriyakumaran (2014) 
between two known Mach number calibration curves. Once the calibration curves for the desired Mach number are established, the measured port pressures are used to calculate $C_{\alpha}$ in Equation 4.4a. Since the $C_{\alpha}$ calibration is a linear function of the flow angle $(\alpha)$, the value of $C_{\alpha}$ can be interpolated to obtain the flow angle $(\alpha)$. The flow angle can then be used to interpolate for the values of $C_{o}$ and $C_{s}$. Equations $4.4 \mathrm{~b}$ and $4.4 \mathrm{c}$ allow the calculation of the total pressure $\left(P_{o 2}\right)$ and static pressure $\left(P_{2}\right)$, and therefore the Mach number. This Mach number is used as the next guess in a subsequent iteration. The iterative process is repeated until the change in Mach number is below $10^{-6}$. Generally, no more than ten iterations are required.

The three-hole probe does not provide accurate measurements of static pressure, particularly at yaw angles near zero, presumably because all three tubes that compose the probe are approximately aligned with the flow and behave roughly as pitot tubes. The curves shown in Figure $4.4 \mathrm{c}$ for the static pressure coefficient $\left(C_{s}\right)$ do not appear in order of increasing Mach number, making interpolation problematic. This issue is overcome by taking measurements of the static pressure directly using the static probe. The static pressure obtained from the three-hole probe is still useful as a point of comparison for aligning the positions of the measurements made by the two probes. This alignment procedure will be presented in Section 4.4.5,

\subsubsection{Rayleigh Shock Correction}

The three-hole probe is only calibrated up to high subsonic Mach numbers. When the three-hole probe encounters supersonic flow, a bow shock wave occurs just upstream of the probe tip as shown in Figure 4.5. The total pressure observed at the probe tip is thus lower than the true total pressure of the measured flow. Fortunately, the total pressure ratio across a normal shock is a well known function of the upstream Mach number. The total pressure measured by the probe can thus be corrected to the true value.

The correction method requires two known values: the total pressure measured by the probe $\left(P_{O P}\right)$ and the true static pressure $\left(P_{2}\right)$, measured separately using the static probe. The unknown value is the true total pressure $\left(P_{o 2}\right)$, ahead of the normal shock. The Rayleigh 


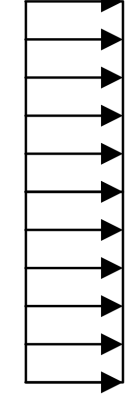

\section{True flow}

$\mathrm{M}_{2}>1.0$

$\mathrm{P}_{02}, \mathrm{P}_{2}$

Flow altered by the presence of the shock

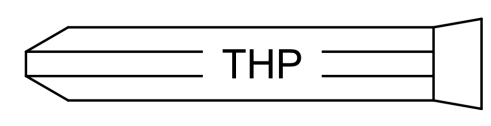

$M_{P}<1.0$

$\mathrm{P}_{\mathrm{OP}}$

Figure 4.5: Flow Quantities around the Probe Tip Shock Wave (Adapted from Sooriyakumaran, 2014)

Pitot Formula describes the relationship between the known quantities $P_{o P}$ and $P_{2}$, and the unknown true Mach number ahead of the normal shock $\left(M_{2}\right)$, as follows:

$$
\frac{P_{o P}}{P_{2}}=\left[\frac{(\gamma+1) M_{2}^{2}}{2}\right]^{\frac{\gamma}{\gamma-1}}\left[\frac{\gamma+1}{2 \gamma M_{2}^{2}-(\gamma-1)}\right]^{\frac{1}{\gamma-1}}
$$

Equation 4.6 can be solved iteratively for the true Mach number. Once this value is known, the total pressure ratio across a normal shock can be obtained from the following expression (White, 2006):

$$
\frac{P_{o P}}{P_{o 2}}=\left(\frac{(\gamma+1) M_{2}^{2}}{2+(\gamma-1) M_{2}^{2}}\right)^{\frac{\gamma}{\gamma-1}}\left(\frac{\gamma+1}{2 \gamma M_{2}^{2}-(\gamma-1)}\right)^{\frac{1}{\gamma-1}}
$$

Using Equation 4.7, the true total pressure $\left(P_{o 2}\right)$ is obtained.

\subsubsection{Alignment Procedures for Three-Hole and Static Pressure Probe Measurements}

As described in Section 3.3.3, the three-hole probe and static probe measurements are performed separately. Despite taking measurements at the same nominal locations, the data from the two probes are generally misaligned in the pitchwise direction. This misalignment occurs due to backlash in the traverse gear and small discrepancies in position when the probes 


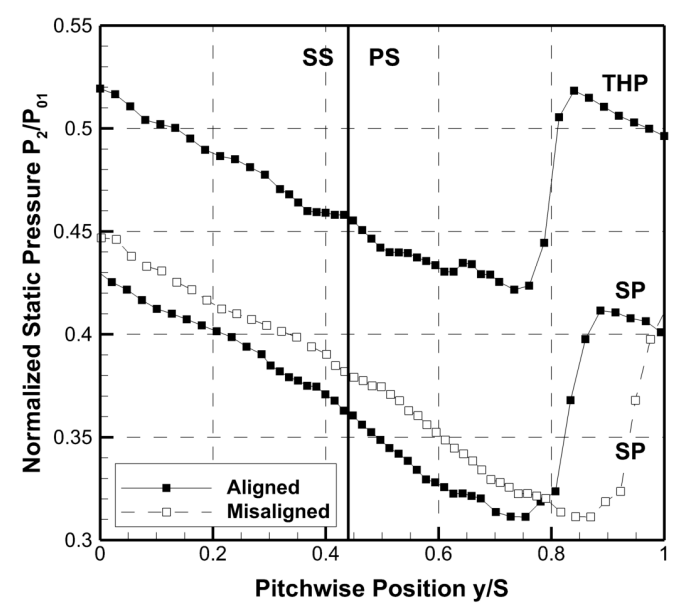

(a) Static Pressure Distributions from the ThreeHole Probe (THP) and the Static Probe (SP)

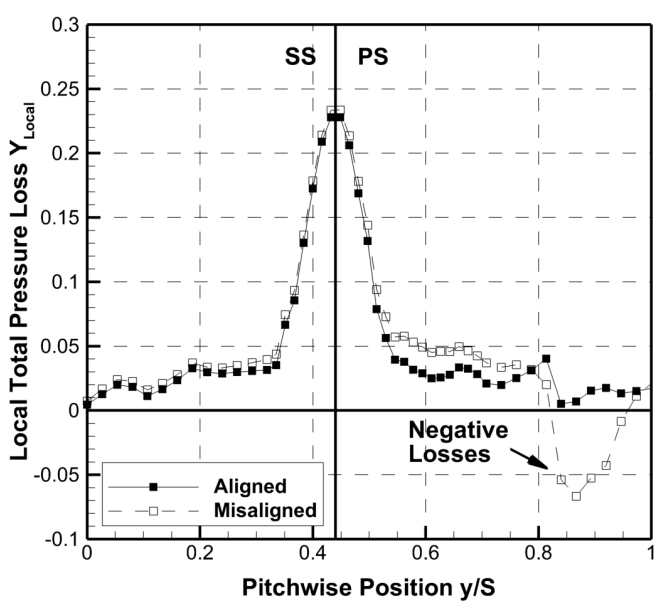

(b) Local Loss Coefficient

Figure 4.6: Static Pressure Pitchwise Misalignment and its Effect on the Local Loss Distribution, at a Mixed-Out Mach Number of 1.25 (Adapted from Sooriyakumaran, 2014)

are mounted manually. Because a normal shock wave occurs at the tip of the three-hole probe, the static pressure obtained from the probe calibration is offset from the true value of static pressure in the undisturbed flow. Despite this offset, the general blade-to-blade static pressure trends are still captured by the three-hole probe. Therefore, the pressure data from the static probe, misaligned in the pitchwise direction, can be realigned by visually comparing them to the pressure data from the three-hole probe.

In order to show the effect of static pressure misalignment on the calculated losses, the pitchwise variations of static pressure and local total pressure loss coefficient $\left(Y_{\text {Local }}\right)$ are shown in Figure 4.6, with an exaggerated static pressure misalignment. The data were obtained for the AF4 cascade at an outlet Mach number of 1.25, and the static pressures are normalized by the corrected inlet total pressure. The local total pressure loss coefficient $\left(Y_{\text {local }}\right)$ is defined as:

$$
Y_{\text {local }}=\frac{P_{o 1}-P_{o}}{P_{o 2}-P_{2}}
$$

where $P_{o 1}$ is the upstream total pressure (corrected according to the method in Appendix A), $P_{o}$ is the local total pressure downstream of the cascade, and $P_{o 2}$ and $P_{2}$ are the reference 
mixed-out total pressure and static pressure, respectively. The concept of "mixed-out" flow conditions will be explained in the next section.

The misaligned static pressure distribution causes regions of apparently higher and lower losses, including a region of negative total pressure losses, which cannot physically occur in the linear cascade since no work is done on the fluid. The erroneous losses primarily result from the Rayleigh shock correction described in the preceding section. If the misaligned static distribution causes a region of static pressure that is lower than the true value, this region is calculated to have an incorrectly high Mach number. The Rayleigh shock correction then overestimates the shock losses at the probe tip, and the remaining blade profile losses are too low. Since this shock correction only takes effect at supersonic Mach numbers, cases at subsonic Mach numbers are not affected by the error introduced by the correction. Misalignment in the static pressure distribution has an additional small effect on the local loss coefficient $Y_{\text {local }}$ and other parameters by affecting the integrated outlet flow conditions. This error is not expected to be large since the static pressure distribution is approximately periodic, meaning that the integrated static pressure across one pitch should not be greatly affected by pitchwise shifting of the distribution.

\subsubsection{Mixing Calculation for Non-Uniform Flow}

As the flow leaves a blade row, it contains non-uniformities such as wakes, separated boundary layers and shocks propagating downstream. As these non-uniformities dissipate through viscous mixing, additional entropy is generated, ultimately causing the total pressure to reduce further. For this reason, the measurements taken at the outlet measuring plane $\left(0.5 C_{x}\right.$ downstream of the trailing edge $)$ are not representative of the final outlet flow because additional mixing loss has yet to take place. It is thus desired to obtain the "mixed-out" flow, in which all flow properties have reached a uniform value through viscous dissipation. Results obtained from this mixing calculation are more easily comparable to data from other researchers because the results are independent of the distance downstream from the cascade.

It is possible to calculate the mixed-out flow parameters using a control volume analysis, as

described by Prassad (2005). This method begins by defining a control volume downstream of 


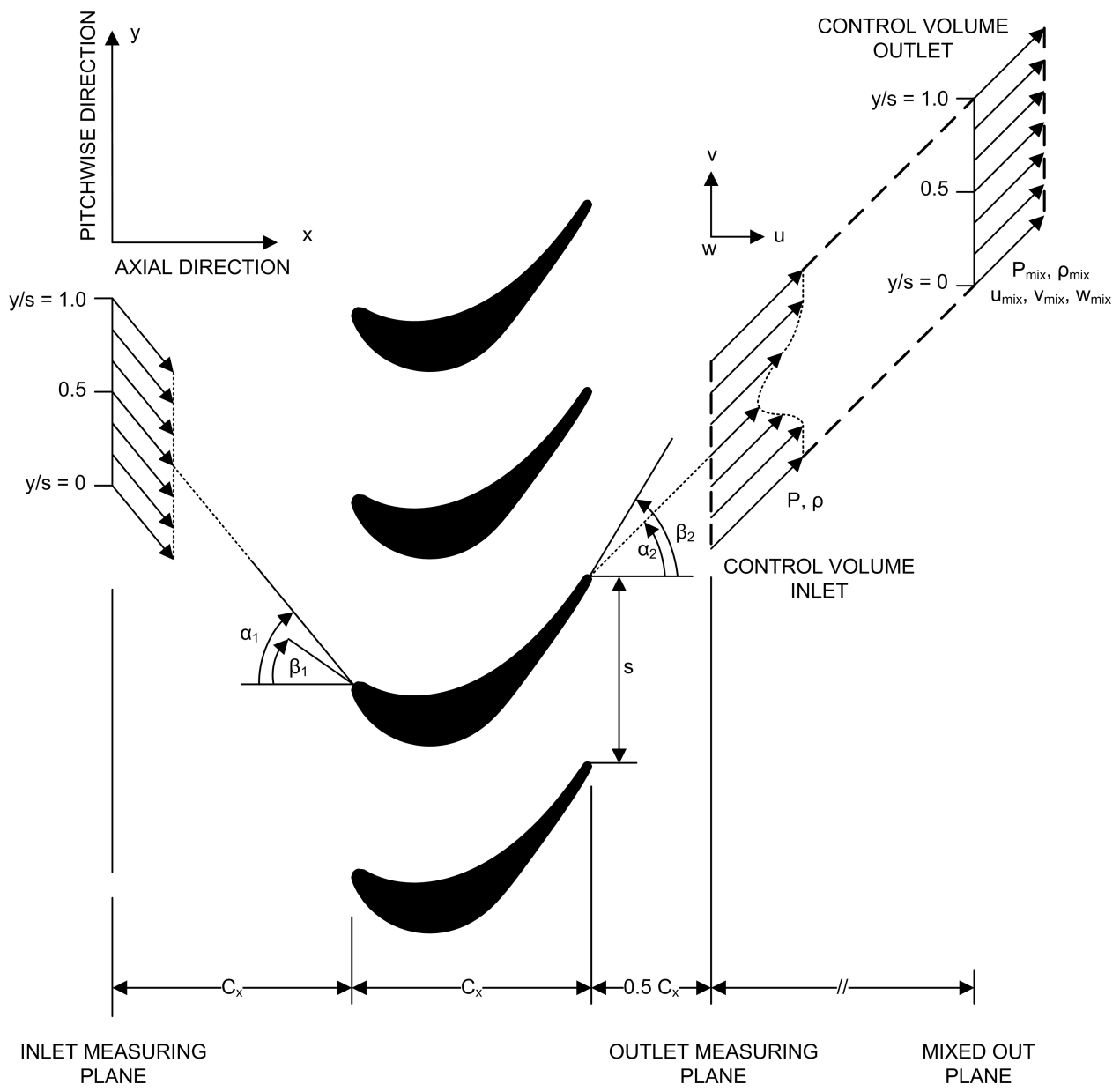

Figure 4.7: Control Volume Used for the Mixing Calculation (Adapted from Sooriyakumaran, 2014) 
the cascade, as shown in Figure 4.7. The control volume inlet is taken directly from the cascade outlet measuring plane, and the control volume outlet is located sufficiently far downstream that flow quantities have become uniform. The control volume has frictionless walls and a constant cross-sectional area so that the flow quantities are only changed by viscous mixing. In order to obtain the flow quantities at the mixed-out plane, the mass, momentum and energy conservation equations are applied between the control volume inlet and outlet. As a first step, the mass flow rate, the momentum flow rate in the $\mathrm{x}-, \mathrm{y}-$ and $\mathrm{z}$-directions, and the energy flow rate are integrated across the control volume inlet as follows:

$$
\begin{aligned}
\dot{m} & =\int \rho u \mathrm{~d} A \\
X & =\int(\rho u) u \mathrm{~d} A+\int P \mathrm{~d} A \\
Y & =\int(\rho u) v \mathrm{~d} A \\
Z & =\int(\rho u) w \mathrm{~d} A \\
E & =\frac{\gamma R}{\gamma-1} \int(\rho u) T \mathrm{~d} A+\frac{1}{2} \int(\rho u)\left(u^{2}+v^{2}+w^{2}\right) \mathrm{d} A
\end{aligned}
$$

where $\dot{m}$ is the mass flow, $\mathrm{X}$ Y Z are the rate of fluid momentum flow entering the control volume and $\mathrm{E}$ is the flow rate of energy entering the control volume. Given that measurements are made at the cascade midspan, the spanwise component of momentum flow (Z) is assumed to be zero. The density $(\rho)$ and static temperature $(T)$ distributions at the measuring plane can be obtained from the measured stagnation conditions using isentropic Mach number relations and the perfect gas law.

Meanwhile, at the control volume outlet plane, all flow quantities have reached uniform conditions, so it is unnecessary to integrate. 


$$
\begin{aligned}
\dot{m} & =\rho_{m i x} u_{m i x} A \\
X & =\dot{m} u_{m i x}+P_{m i x} A \\
Y & =\dot{m} v_{m i x} \\
Z & =\dot{m} w_{m i x} \\
E & =\frac{\gamma \dot{m} P_{m i x}}{(\gamma-1) \rho_{m i x}}+\frac{\dot{m}}{2}\left(u_{m i x}^{2}+v_{m i x}^{2}+w_{m i x}^{2}\right)
\end{aligned}
$$

The subscript mix in Equations 4.10 refers to mixed-out quantities at the control volume outlet. Solving Equations 4.10 for the mixed-out pressure $\left(P_{\text {mix }}\right)$ yields the following quadratic equation:

$$
Q P_{m i x}^{2}+L P_{m i x}+C=0
$$

where

$$
\begin{aligned}
Q & =\frac{A^{2}}{\dot{m}^{2}}\left(1-\frac{2 \gamma}{\gamma-1}\right) \\
L & =\frac{2 X A}{\dot{m}^{2}}\left(\frac{\gamma}{\gamma-1}-1\right) \\
C & =\frac{1}{\dot{m}^{2}}\left(X^{2}+Y^{2}+Z^{2}\right)-\frac{2 E}{\dot{m}}
\end{aligned}
$$

Once the mixed-out pressure $\left(P_{m i x}\right)$ is obtained, it is back-substituted into Equations 4.10 to calculate the remaining mixed-out variables.

Inherent in this analysis is the assumption that no heat transfer occurs in the wind tunnel test section, resulting in a constant total temperature across the cascade. Jouini (2000) performed a simplified heat transfer analysis for a cascade in the high-speed wind tunnel. Using estimates of the highest and lowest expected wind tunnel wall temperatures, it was found that the variation in total pressure loss coefficient (Y) and in axial-velocity-density ratio (AVDR) as a result of the heat transfer was within the estimated uncertainty for these variables. For this reason, the wind tunnel test section is assumed adiabatic. 


\subsection{Measurement Uncertainty}

\subsubsection{Introduction}

An assessment of the uncertainty of data collected in the high-speed wind tunnel was performed by Corriveau (2005), and subsequently by Hall (2012) for lower Mach numbers. Both authors employed the methods prescribed by the AIAA Standard S-071A-1999 titled "Assessment of Experimental Uncertainty with Application to Wind Tunnel Testing". These assessments are still valid, and the methods used to obtain the uncertainty estimates are summarized below.

\subsubsection{Methodology}

The uncertainty assessment method supposes a calculated variable $(F)$, which is a function of $\mathrm{N}$ independent variables $\left(\phi_{i}\right)$.

$$
F=F\left(\phi_{1}, \phi_{2}, \ldots \phi_{N}\right)
$$

Each variable $\left(\phi_{i}\right)$ is composed of its true value $\left(\hat{\phi}_{i}\right)$ and an error $\left(\delta \phi_{i}\right)$.

$$
\phi_{i}=\hat{\phi}_{i}+\delta \phi_{i}
$$

Error in the independent variables generates an error in the calculated variable, which can be estimated as follows:

$$
(\delta F)_{i}=\left(\frac{\partial F}{\partial \phi_{i}}\right) \delta \phi_{i}
$$

Assuming that the errors due to the independent variables $\left(\delta \phi_{1}, \delta \phi_{2}, \ldots \delta \phi_{N}\right)$ are normally distributed, the error in the calculated variable can be expressed as:

$$
\delta F=\sqrt{\left(\frac{\partial F}{\partial \phi_{1}}\right)^{2} \delta \phi_{1}^{2}+\left(\frac{\partial F}{\partial \phi_{2}}\right)^{2} \delta \phi_{2}^{2}+\ldots+\left(\frac{\partial F}{\partial \phi_{N}}\right)^{2} \delta \phi_{N}^{2}}
$$


There exist two types of error: bias (fixed/systematic) error and precision (random) error (Tavoularis, 2005). Bias errors result from the measurement method, and are constant throughout the experiment. They can sometimes be estimated and corrected for, but otherwise contribute to the measurement uncertainty. Precision errors result from the seemingly random effect of small unintended disturbances to the experiment and measurement system.

The errors due to the independent variables $\left(\delta \phi_{1}, \delta \phi_{2}, \ldots \delta \phi_{N}\right)$ can be either bias or precision errors. In the case of bias errors, the value of a particular uncertainty contribution $\delta \phi$ can be obtained from manufacturer specifications for the measuring device, from analytical considerations or from experience. The value for a particular precision uncertainty contribution can be obtained by repeating measurements and obtaining the standard deviation from a sample of size $N>10$. The value of $\delta \phi$ is twice the standard deviation in order to have a $95 \%$ confidence interval.

\subsubsection{Uncertainty in Pressure Measurements}

Pressure data are used in the calculation of every type of wind tunnel result. In order to assess the uncertainty in these results, the uncertainty in pressure data must first be quantified. Pressure data are obtained based on the voltage output of the pressure transducers, using the following linear curve fit:

$$
P=m V+b
$$

$P$ is the pressure, $V$ is the transducer output voltage, $m$ is the slope of the calibration curve, and $b$ is the intercept of the calibration curve. These transducer calibration curves were presented in Figure 4.4. Applying Equation 4.16, the error in the pressure measurement is given by the following expression:

$$
\delta P=\sqrt{\left(\frac{\partial P}{\partial m}\right)^{2} \delta m^{2}+\left(\frac{\partial P}{\partial V}\right)^{2} \delta V^{2}+\left(\frac{\partial P}{\partial b}\right)^{2} \delta b^{2}}
$$

which can be developed into:

$$
\delta P=\sqrt{(V \delta m)^{2}+(m \delta V)^{2}+\delta b^{2}}
$$




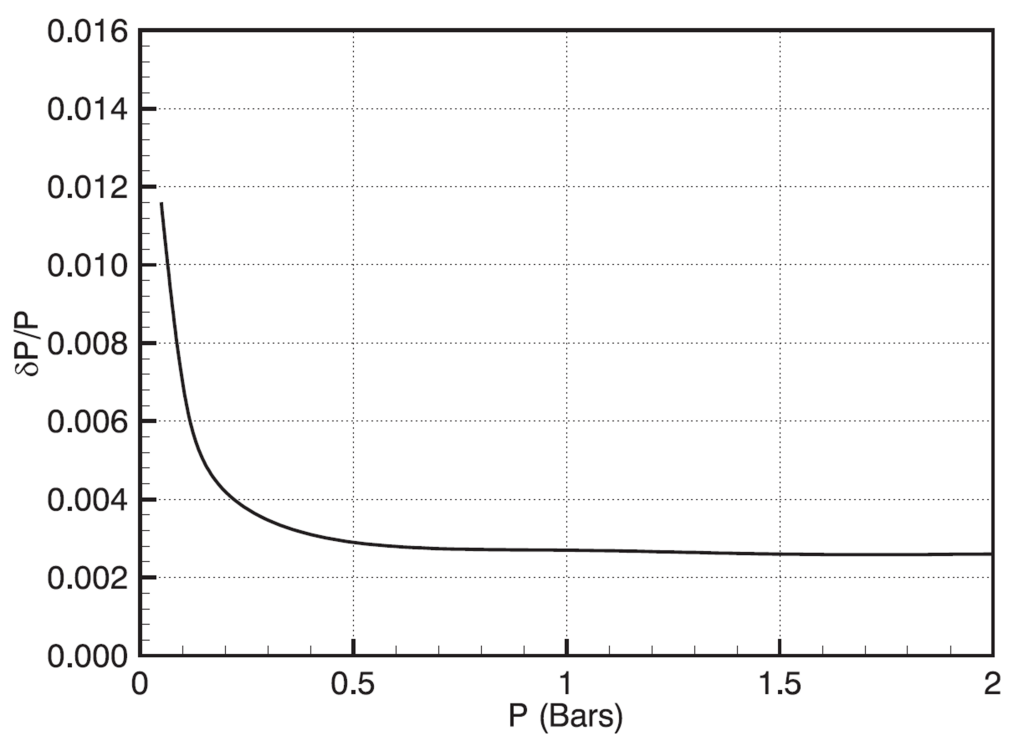

Figure 4.8: Variation of Uncertainty on Pressure Measurements (Corriveau, 2005)

Eleven different pressure transducers were employed in this study (as described in Section 3.3.1), and the estimated uncertainty was found to be similar for all transducers. The uncertainty in the voltage signal was estimated by Jeffries (2000) to be $0.25 \%$ of the absolute value of voltage, based on a 0.1 second sampling time and a sampling frequency of 2000 Hz. The uncertainty in the calibration curve slope $\delta m$ was obtained from the transducer calibration data from Sooriyakumaran (2014). The calibration curve offset $\delta b$ tended to drift over time, presumably due to changing ambient conditions in the laboratory throughout the seasons. This uncertainty was a known bias and was accounted for by measuring the correct offset $(b)$ at the beginning of every testing day. Using Equation 4.19 , the uncertainty in pressure measurements was obtained, as shown in Figure 4.8. The largest values of uncertainty occur at low pressures. Since the pressures encountered in the wind tunnel did not fall below 0.5 bar, the uncertainty can be taken as approximately constant, with a value of $\pm 0.3 \%$.

\subsubsection{Uncertainty in Calculated Quantities}

The methodology described above was repeated for other calculated quantities which are based on the pressure data. The results of the uncertainty analysis by Corriveau and Hall are provided in Table 4.1 . 
Table 4.1: Uncertainty of Measured and Calculated Values (Corriveau, 2005; Hall, 2012)

\begin{tabular}{lcc}
\hline & Range & Uncertainty Range \\
\hline \hline Pressure, $P$ & 0.5 to 1.5 bars & $\pm 0.3 \%$ \\
Inlet Flow Angle, $\alpha_{1}$ & Design Incidence $0^{\circ}$ & $\pm 1.0^{\circ}$ \\
Outlet Flow Angle, $\alpha_{2}$ & $\pm 10^{\circ}$ relative to probe axis & $\pm 0.5^{\circ}$ \\
Inlet Mach number, $M_{1}$ & $<0.6$ & \pm 0.008 \\
Outlet Mach number, $M_{2}$ & 0.8 to 1.3 & \pm 0.005 \\
Loss Coefficient, $Y$ & $M_{2, \text { is }}$ from 0.5 to 1.25 & \pm 0.020 to \pm 0.007 \\
Reynolds Number, $R e$ & $M_{2, \text { is }}$ from 0.5 to 1.25 & \pm 6000 to \pm 97000 \\
\hline
\end{tabular}

The Reynolds number depends on the pressure and temperature of the flow. It was demonstrated by Corriveau (2005) that the uncertainty in Reynolds number obtained using Equation 4.16 ranged from \pm 6000 to $\pm 12,000$, at the lowest and highest outlet isentropic Mach numbers encountered, respectively. This Reynolds number is based on the averaged static temperature during a run. However, as explained in Jeffries (2000), over the course of a run, the total temperature in the wind tunnel decreases due to the expansion of the air in the storage tanks. Using the range of temperatures encountered over the course of a run, Corriveau (2005) calculated new uncertainty estimates for the Reynolds number, which are the values shown in Table 4.1. The Reynolds number variation introduced by the temperature decrease in the wind tunnel was much larger than the uncertainty obtained from Equation 4.16 . The variation in Reynolds number is largest at high Mach numbers because the greater air expansion in the storage tanks causes a greater temperature decrease over the course of a run. Fortunately, at high Reynolds numbers such as those encountered in the high Mach number cases, the Reynolds number has a weak influence on the profile losses. It was shown by Jouini (2000) that the uncertainty in profile losses introduced by the change in Reynolds number was less than the estimated uncertainty due to all other sources.

The experimental procedures and measurement uncertainty have now been fully presented. The next chapter will describe the procedures used to generate numerical simulations of the cascade flows. The following chapter will cover the results and discussion of both the experimental and numerical approaches. 


\section{Chapter 5}

\section{Computational Procedures}

\subsection{Introduction}

The experiments in the high-speed wind tunnel provide valuable measurements of the physical processes occurring in the flow. However, there are certain regions in the flow that cannot be measured due to practical limitations, such as the geometry of the probe traverse mechanism and the interference caused by the probe blockage near blade surfaces. By simulating the flow conditions using Computational Fluid Dynamics (CFD), it becomes possible to examine the entire flow field around the blade. The simulations also have their shortcomings, such as the assumption of steady-state Reynolds-averaged flow, the use of a turbulence model, a transition model and idealized boundary conditions. It is acknowledged that CFD does not replace the need for experimental measurements, but it is a valuable tool.

In order to complement the measurements in the wind tunnel, the experimental conditions for the AF4 cascade were recreated in CFD using the ANSYS CFX package. Although the HS1 cascade family was not tested experimentally in the present study, numerical simulations of the HS1 cascades were also performed and compared to experimental data from Corriveau (2005).

The current chapter provides the procedures used in the numerical simulations of the AF4 cascade and HS1 family of cascades. First, the CFD solver is described, including the choices of turbulence and transition models. The fluid domain geometry and mesh topology 
are then described. Finally, the boundary conditions which are applied to the fluid domain are presented.

\subsection{Solver}

The numerical simulations were performed using ANSYS CFX version 15.0. The software uses a finite volume method to solve the Navier-Stokes equations iteratively. The method begins by constructing control volumes around the nodes of a mesh provided by the user. The governing equations are then integrated over each control volume and discretized. The solution variables are stored at the nodes of the control volumes. In order to obtain the values of these variables at the faces of the control volumes, values are interpolated between nodes. A shape factor is assigned based on the element type. In order to deal with the advection terms, the specified blend factor option was selected for all simulations. The blend factor was set to 1 , which indicates a central difference scheme. This option is second order accurate in space.

The Shear Stress Transport (SST) turbulence model is a two-equation eddy viscosity model (ans, 2013). The SST model combines the best aspects of the k-omega $(k-\omega)$ and k-epsilon $(k-\epsilon)$ turbulence models. Transport equations are used to obtain the turbulence kinetic energy $(k)$, the turbulence frequency $(\omega)$ and the turbulence eddy dissipation rate $(\epsilon)$. A blending function is then used to favour the k-omega model near the blade surface and the k-epsilon model in the freestream. The SST turbulence model was selected since it is claimed to give an accurate prediction of separation. This aspect was considered particularly important for base pressure predictions in the separated flow region behind the trailing edge.

The gamma-theta transition model was employed to predict transition from laminar to turbulent flow. The model uses two additional transport equations to obtain the turbulence intermittency $(\gamma)$ and the transition momentum thickness Reynolds number $\left(R e_{\theta t}\right)$. The model has been validated with the SST turbulence model and is based on an empirical correlation by Langtry \& Mentor 2005). 


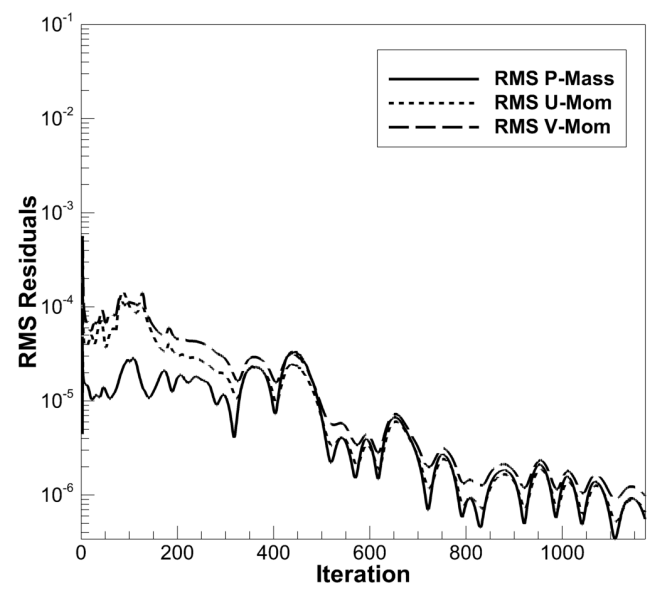

(a) Normalized Residuals of Mass Flow and Momentum

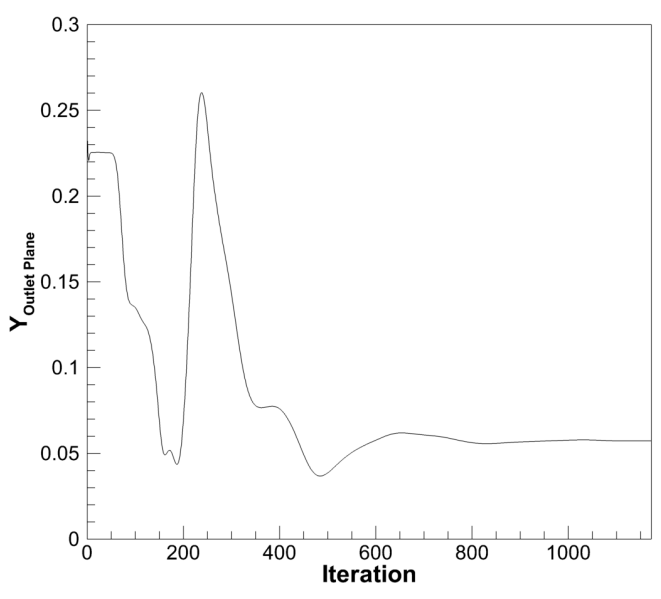

(b) Predicted Profile Losses

Figure 5.1: Typical Convergence Behaviour of the Simulations

All simulations were steady-state, and air was treated as an ideal gas. The total energy heat transfer model was employed with the viscous work term enabled, as recommended by the CFX documentation for turbomachinery flows (ans, 2013). Simulations were considered converged when the normalized RMS residuals were below $10^{-5}$. In addition to monitoring the residuals, the predicted profile losses were calculated based on the conditions at the inlet and outlet of the fluid domain. The total pressure loss coefficient was calculated as follows:

$$
Y_{\text {OutletPlane }}=\frac{P_{01}-P_{02}}{P_{02}-P_{2}}
$$

where the total pressure values were mass averaged and static pressure values were area averaged, based on the recommendations in Cumpsty \& Horlock (2006). In Figure 5.1, convergence plots of the normalized residuals and the predicted profile losses are shown for the AF4 cascade. The behaviour shown is typical of simulations for all four cascades. 


\subsection{Mesh}

Four meshes, one for each cascade, were created in ICEM CFD 15.0. The meshes were unstructured and employed hexahedral elements. ANSYS CFX 15.0 is a three-dimensional solver and, strictly speaking, cannot process two-dimensional meshes. Given that only profile losses were examined, the meshes were three nodes thick in the spanwise direction, and data were taken from the midplane nodes. The meshes had roughly 800000 nodes. A grid refinement study was performed for all four cascades, with comparable results. The results for the AF4 cascade are shown in Figure 5.2. Noting the truncated axis in Figure 5.2(a), the total pressure losses are seen to be nearly converged at about 350000 nodes. However, other monitored parameters such as the outlet flow angle and the integrated blade lift did not converge until after about 650000 nodes. The 800000 node mesh was therefore selected.

The mesh blocking is illustrated in Figure 5.3 for the AF4 cascade, showing an O-grid topology around the blade and an H-grid topology throughout the rest of the mesh. This approach was used for all four cascades. The fluid domain length and the location of the measurement plane are also indicated in Figure 5.3. The measurement plane is where wake traverse data were extracted from the simulation and processed using the same mixing-out calculation as the experimental data, as described in Section 4.4.6. A detailed view of the inflation layers at the trailing edge is shown in Figure 5.4. The mesh quality was assessed on the basis of several parameters: the determinant, the minimum internal angle and the volume change. The determinant refers to the normalized determinant of the Jacobian of each element. The volume change refers to the ratio of the volume of the largest adjacent element to the volume of the element in question. The quality of the mesh in the near-wall region (in this case, at the blade surface) was assessed based on the the $y^{+}$parameter and the boundary layer expansion ratio. The $y^{+}$parameter is defined as follows.

$$
y^{+}=\frac{\rho_{w} \sqrt{\frac{\tau_{w}}{\rho_{w}}} y}{\mu_{w}}
$$




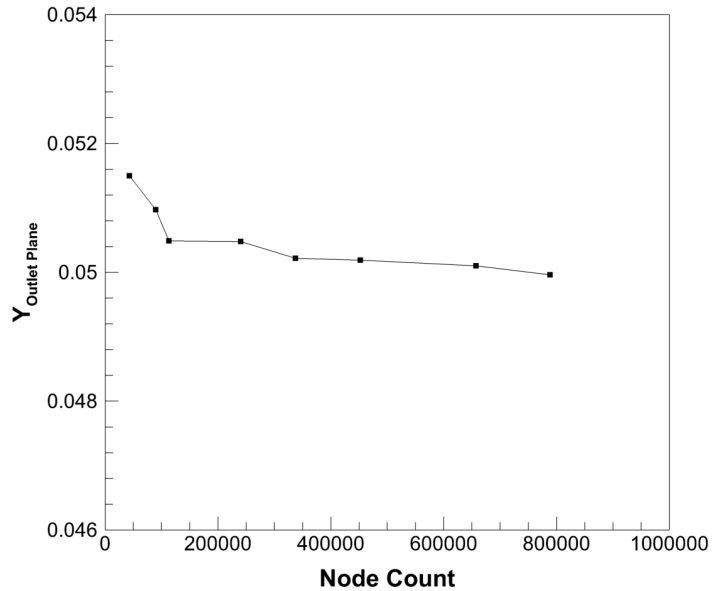

(a) Total Pressure Loss Coefficient

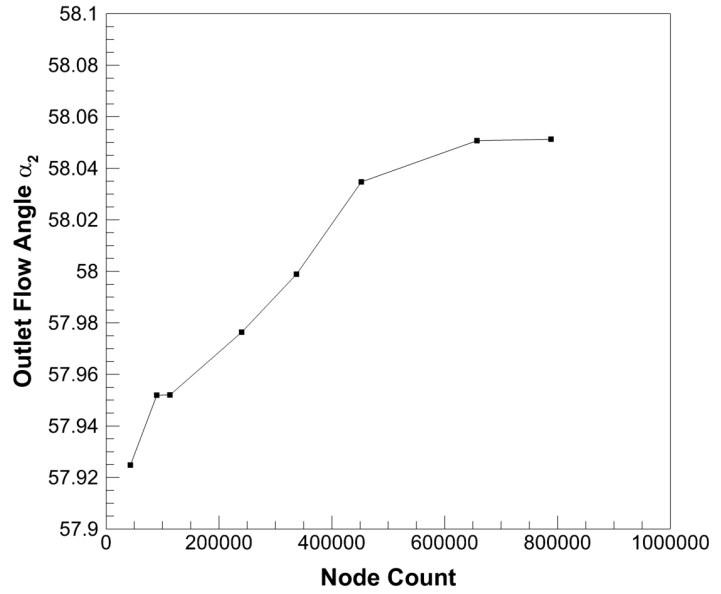

(b) Outlet Flow Angle

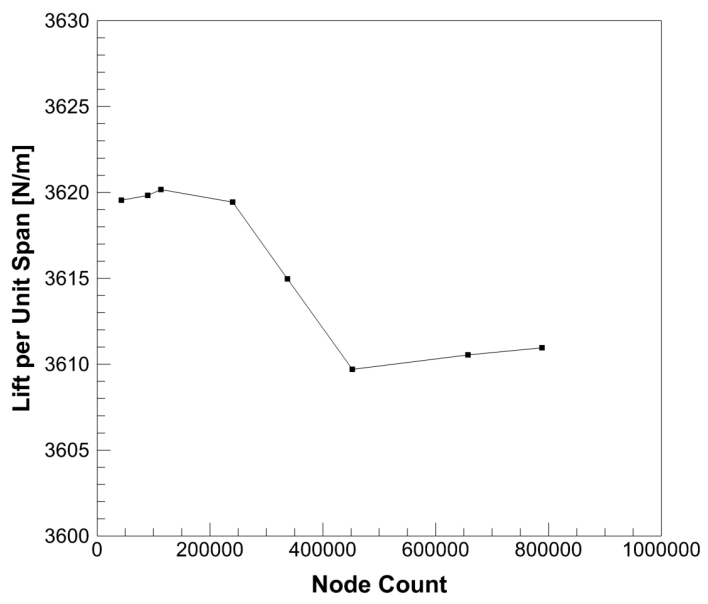

(c) Lift per Unit Span

Figure 5.2: Grid Convergence Study for the AF4 Airfoil 
Table 5.1: Mesh Quality Criteria for the Four Cascades

\begin{tabular}{lcc}
\hline Criterion & Recommended Value & Mesh Quality Achieved \\
\hline Determinant & $>0.3$ & $>0.8$ \\
Minimum Internal Angle & $>20^{\circ}$ & $>27^{\circ}$ \\
Volume Change & $<2$ & $<1.9$ \\
$y^{+}$of first node from the wall & $\sim 1$ or $<5$ & $<3$ \\
Boundary Layer Expansion Ratio & $\leq 1.1$ & 1.06 \\
\hline
\end{tabular}

In Equation 5.2 , the subscript $w$ denotes a value at the wall, and the variable $y$ is the normal distance from the wall. Since $\sqrt{\frac{\tau_{w}}{\rho_{w}}}$ has units of velocity (the term is known as the shear velocity), $y^{+}$is effectively a Reynolds number. In order for the gamma-theta transition model to be applicable, $y^{+}$should be approximately equal to $y^{+} \leq 1$. Values of $y^{+}$that are too high $(>5)$ cause the location of transition to move upstream (Langtry \& Mentor, 2005). Lastly, it is recommended that the expansion ratio throughout the domain have a value of $\leq 1.1$. Efforts were made to ensure that all parameters were within the recommended guidelines for ANSYS CFX. The mesh quality criteria achieved are given in Table 5.1 .

\subsection{Boundary Conditions}

The boundary conditions applied to the fluid domain are shown in Figure 5.5. The inlet was defined by a fixed total pressure, with values selected based on experimental data from the wind tunnel. The inlet was assumed to be subsonic. The flow angle was specified according to the design incidence of each cascade. The total temperature was fixed at 285 K. In order to emulate the turbulence conditions encountered in the wind tunnel, the inlet turbulence intensity was set to $4 \%$ and the eddy length scale was set to $1.5 \mathrm{~cm}$, according to the measurements made by Corriveau (2005).

The outlet was defined by a static pressure, taken as the average over the whole outlet. The combination of inlet total pressure and outlet static pressure allowed an outlet isentropic Mach number to be calculated as an initial estimate for the operating point. 


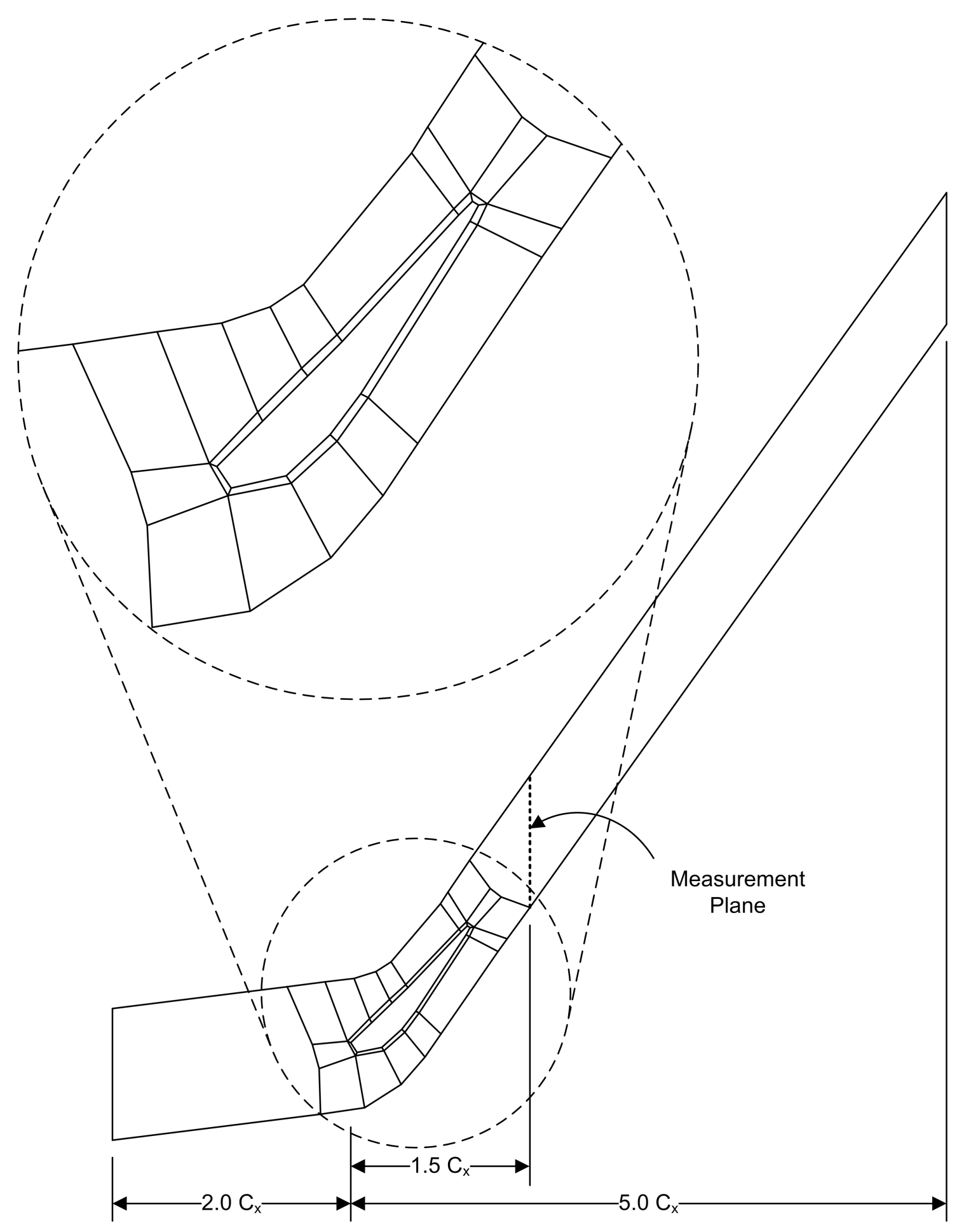

Figure 5.3: Mesh Blocking Topology 


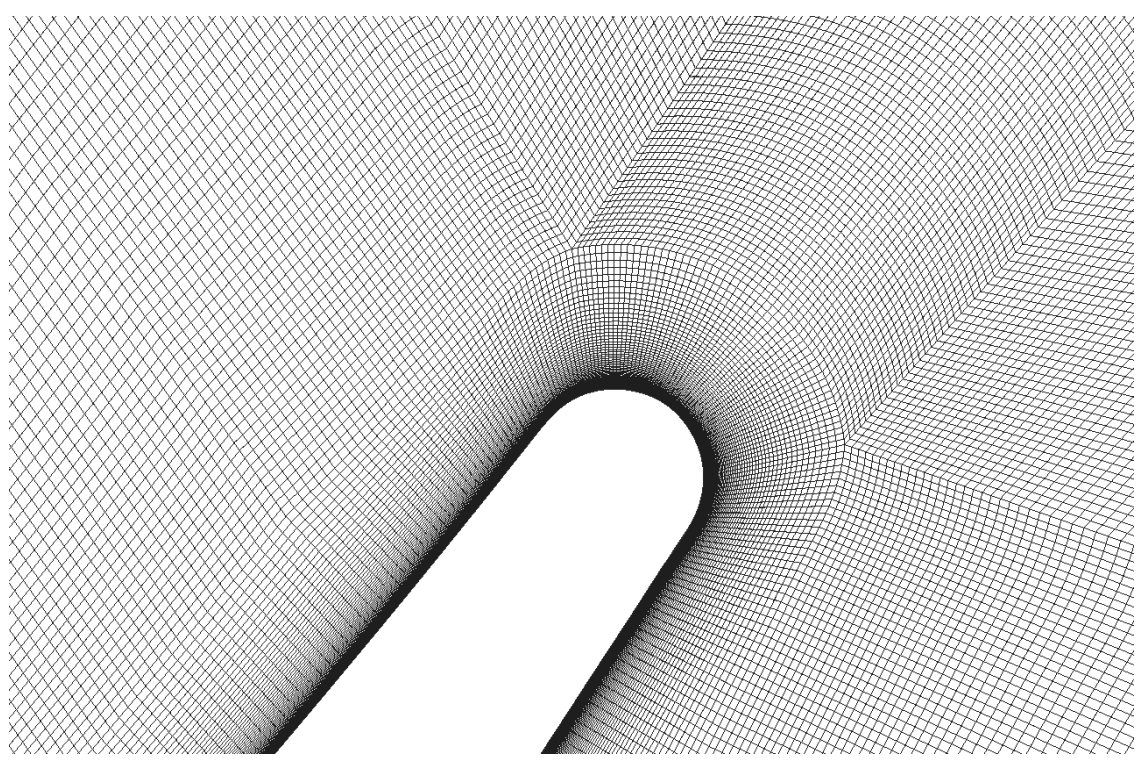

Figure 5.4: Trailing Edge Mesh Detail

The blade surface was defined as a smooth, no slip wall. The wall was assumed adiabatic, as justified in Section 4.4.6 based on a heat transfer analysis performed by Jouini (2000).

The spanwise boundaries were defined as symmetry, which is equivalent to having a blade with infinite span. This choice is appropriate since the flow around a two-dimensional blade was desired in order to obtain the profile losses. The pitchwise boundaries were defined using translational periodicity, with a General Grid Interface (GGI) mesh connection. The GGI connection is used when the node placement on either side of two connected surfaces does not match (ans, 2013).

The numerical simulations performed using the above methods complement the experimental data collected in the wind tunnel. In the next chapter, the results from the experiments and the simulations will be compared, then used to better understand the behaviour of flow around turbine blades at transonic and supersonic Mach numbers. 


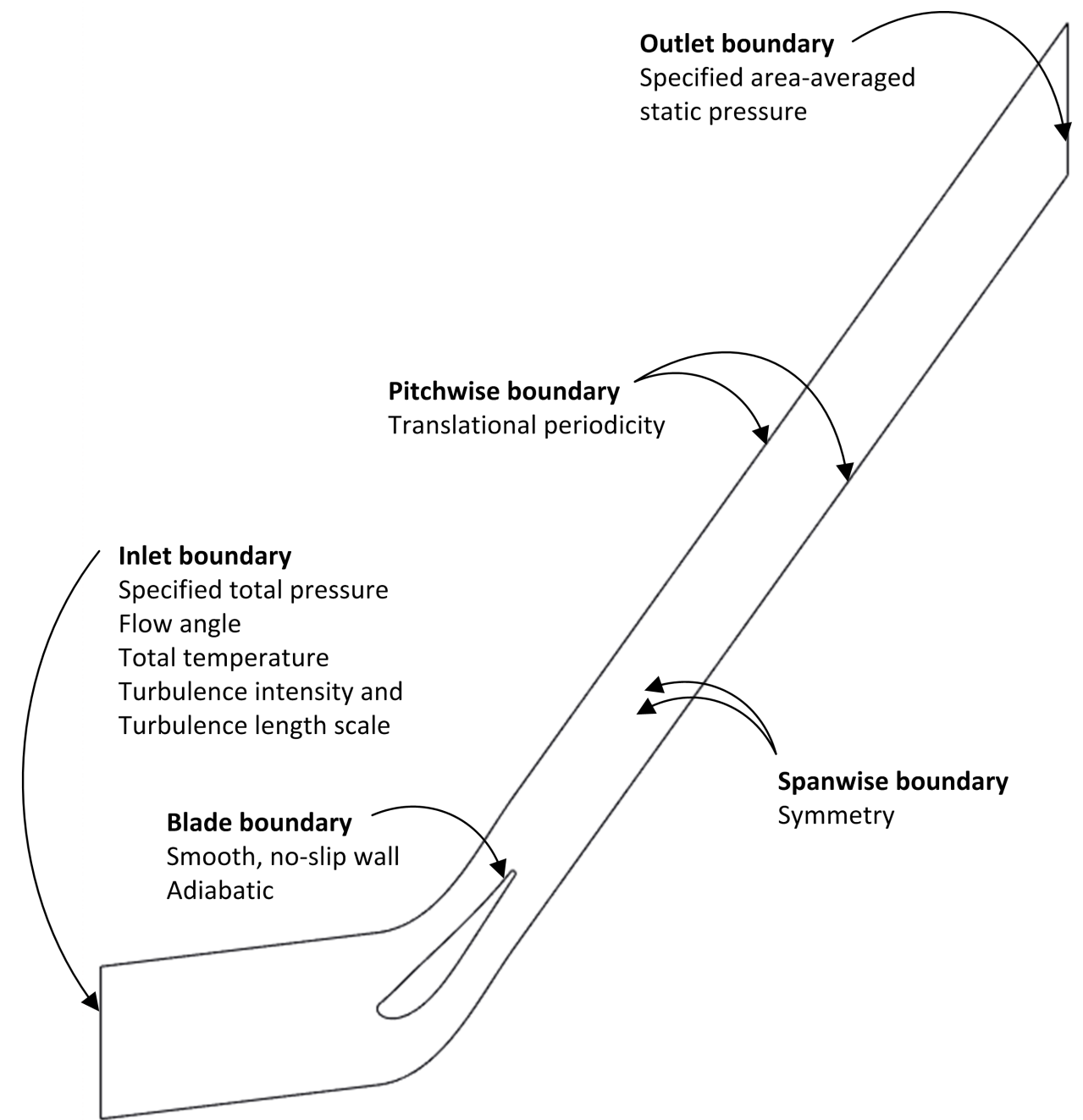

Figure 5.5: Boundary Conditions Applied to the Fluid Domain 


\section{Chapter 6}

\section{Experimental and Computational Results}

\subsection{Introduction}

The current chapter presents the experimental and computational results for the four cascades: AF4, HS1A, HS1C and HS1D. Firstly, an assessment is made of the flow quality, based on the inlet flow uniformity and the outlet flow periodicity. The experimental and computational blade loadings for the four cascades are then compared. The profile losses and the outlet flow angles of the four cascades are presented, followed by the base pressure results. In order to visualize the flow behaviour associated with the profile losses and base pressures, computed contour plots of the Mach number are shown for the four cascades. Boundary layer data are extracted from the simulations. Finally, the base pressure and boundary layer data are used in order to assess a trailing edge loss expression available in the literature.

Many of the results presented in this investigation are related to the same flow features. For this reason, much of the meaningful discussion is deferred to later in the chapter, where enough of the results have been presented to create a complete picture of the flow physics and to avoid repetition. 


\subsection{Flow Quality}

\subsubsection{Introduction}

In order to be confident in the validity of wind tunnel measurements, it is necessary to assess the flow quality. When the high-speed wind tunnel was first commissioned, Jeffries (2000) performed measurements on a well-known blade geometry that had been used to compare the performance of four European transonic cascade wind tunnels (Baines et al., 1986; Kiock et al. 1986). The study by Jeffries (2000) demonstrated that the wind tunnel at Carleton University is capable of producing data of comparable quality to the European wind tunnels.

Prior to taking aerodynamic measurements of a cascade, there are specific flow quality requirements that must be satisfied. In the current investigation, the term flow quality refers to two conditions: the pitchwise flow uniformity across the test section inlet and midspan pitchwise flow periodicity across the test section outlet. For the particular case of midspan measurements in a two-dimensional cascade, the axial velocity density ratio (AVDR) is obtained at the cascade midspan as an approximate indicator of the flow two-dimensionality. The current section demonstrates that all of these flow quality criteria are satisfied reasonably well for the AF4 cascade used in this study.

\subsubsection{Inlet Pitchwise Flow Uniformity}

The inlet flow uniformity is verified by checking that the pitchwise static pressure distribution is uniform across the inlet. Measurements are made using the endwall static pressure taps upstream of the cascade. The static pressure distribution is presented in the form of the inlet Mach number distribution in Figure 6.1 at an isentropic outlet Mach number of $M_{2, i s}=1.3$. The Mach number is approximately constant across the inlet, with the exception of the right-most blade passage. This non-uniformity is likely the result of a flow disturbance upstream, which propagates along the right sidewall boundary layer. This non-uniformity is considered acceptable since the flow is essentially uniform over the central blade passages. 


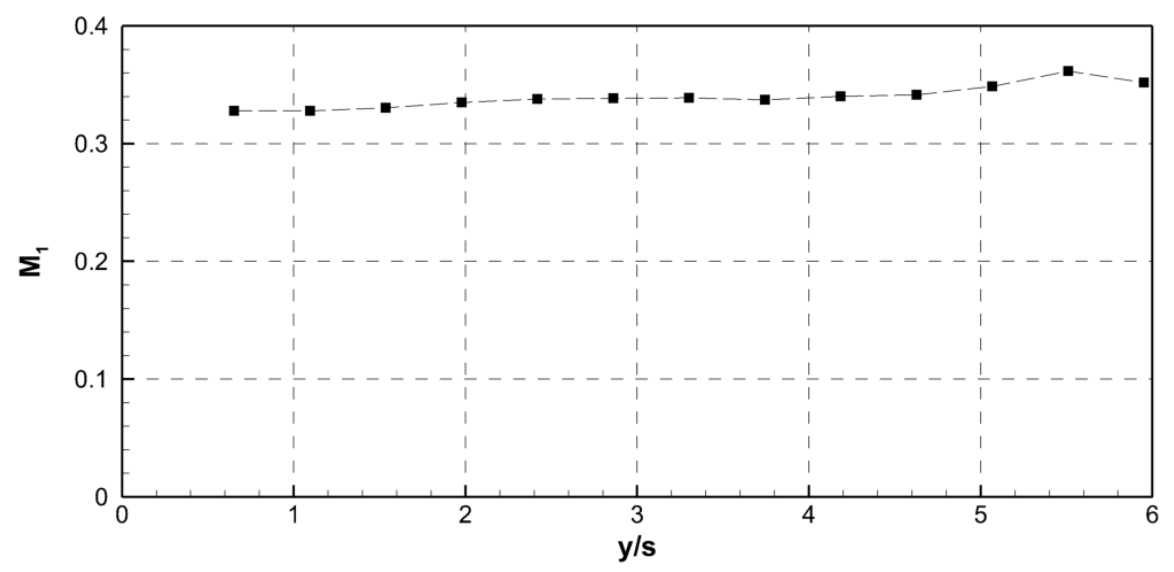

Figure 6.1: Inlet Mach Number Distribution at $M_{2, i s}=1.3$

\subsubsection{Outlet Flow Periodicity}

Since the cascade testing involves measuring flow over only one pitch, it is important to verify that the flow is periodic. The periodicity of the cascade flow can be judged by examining the blade wakes. Using both the three-hole probe and the static probe, wake data are processed for all five blades using the data reduction procedures described in Section 4.4 . The local total pressure loss coefficients $\left(Y_{\text {local }}\right)$ for the five blade wakes are shown in Figure 6.2. The local total pressure loss coefficient is defined as follows:

$$
Y_{\text {local }}=\frac{P_{o 1}-P_{o}}{P_{o 2}-P_{2}}
$$

where $P_{o 1}$ is the upstream total pressure (obtained using the method outlined in Appendix A), $P_{o}$ is the local downstream value of total pressure and $P_{o 2}$ and $P_{2}$ are the mixed-out values of total pressure and static pressure, respectively. Since the wind tunnel can only have a finite number of blades, the side walls that bound the two ends of the blade row generate boundary layers which affect the flow around the outboard blades. Because wake measurements for this study are performed on the central blade (Blade 3), the lower peak of losses observed for blade 5 is not a concern. The mismatches that occur in between blades result from mismatches in the flow conditions between the end of one run and the beginning of the next run. Since the data in Figure 6.2 were collected at a nominal $M_{2, i s}=1.2$, oblique shock waves form 


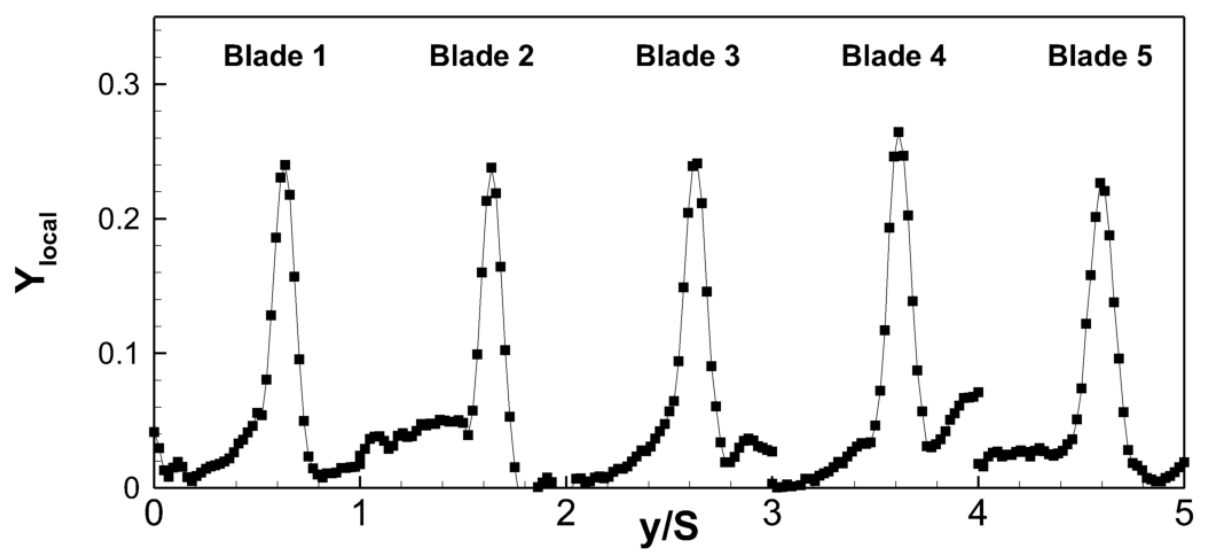

Figure 6.2: Local Total Pressure Loss Coefficient across the Full Cascade at $M_{2, i s}=1.2$

at the trailing edges of the blades and at the cascade end-blocks. The intersection points between these shocks and the measurement plane are sensitive to the outlet Mach number. Although efforts are made to collect data only when the control system has reached a steadystate operating point, the outlet Mach number can decrease slightly near the end of a run as a result of decreasing tank pressure. For wake data collected outside of this periodicity assessment, this issue occurs to a lesser extent because shorter, more conservative runs are performed. In Section 4.3.1 it was seen that a single blade pitch is traversed over the course of three separate runs.

The mixed-out profile losses for the five blades are given in Figure 6.3. The variation in total pressure loss coefficient between the blades in Figure 6.3 are within the quoted measurement uncertainty presented in Section 4.5 .

\subsubsection{Axial Velocity Density Ratio (AVDR)}

The current investigation is on profile losses, which would ideally involve the measurement of a two-dimensional flow around the blade profiles. In reality, the blade-to-blade pressure gradient tends to alter the thickness of the endwall boundary layers across each blade pitch. The higher pressure near the pressure surface tends to thin the endwall boundary layer by driving the low momentum fluid in the boundary layer towards the suction surface. The endwall boundary layer thus tends to become thicker near the suction surface. As a result, the 


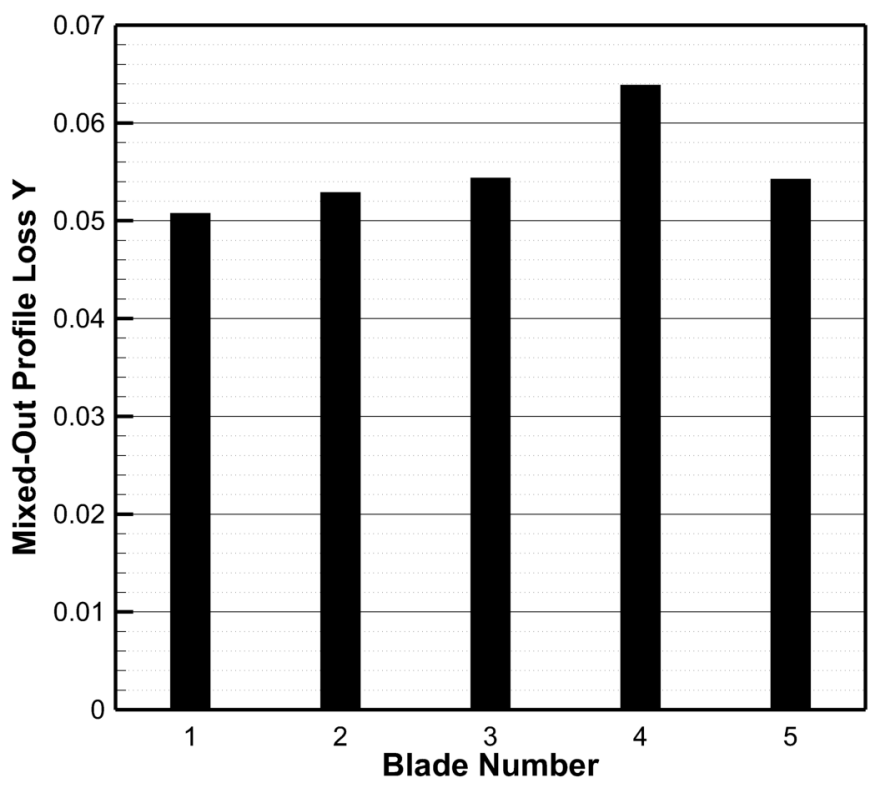

Figure 6.3: Periodicity of the Mixed-Out Profile Loss Coefficient of the AF4 Cascade at $M_{2, i s}=1.2$

flow at the blade midspan converges or diverges, because the altered boundary layer thickness effectively narrows or widens the flow path. The flow is therefore not two-dimensional at midspan.

It is however possible to estimate the pitchwise-averaged flow two dimensionality using the Axial Velocity Density Ratio (AVDR). The AVDR is defined as follows:

$$
A V D R=\frac{\int_{0}^{1}\left(\rho_{2} V_{x, 2}\right)_{M S} \mathrm{~d}(y / s)}{\int_{0}^{1}\left(\rho_{1} V_{x, 1}\right)_{M S} \mathrm{~d}(y / s)}
$$

As described in Section 2.5.5, the AVDR is the ratio of mass flow rate per unit area at the outlet and at the inlet. Since the AVDR is measured at midspan, it is an indicator of the flow convergence or divergence at midspan, averaged across one blade pitch. The AVDR measurements for the AF4 cascade are shown in Figure 6.4. The values are in the range of roughly 0.93 to 0.97 , suggesting an average decrease in mass flow per unit area at midspan. This overall midspan flow divergence may be caused by the large flow acceleration through the blades $\left(M_{1}=0.3\right.$ to $\left.M_{2, i s}=1.2\right)$, which could be thinning the endwall boundary layers. 


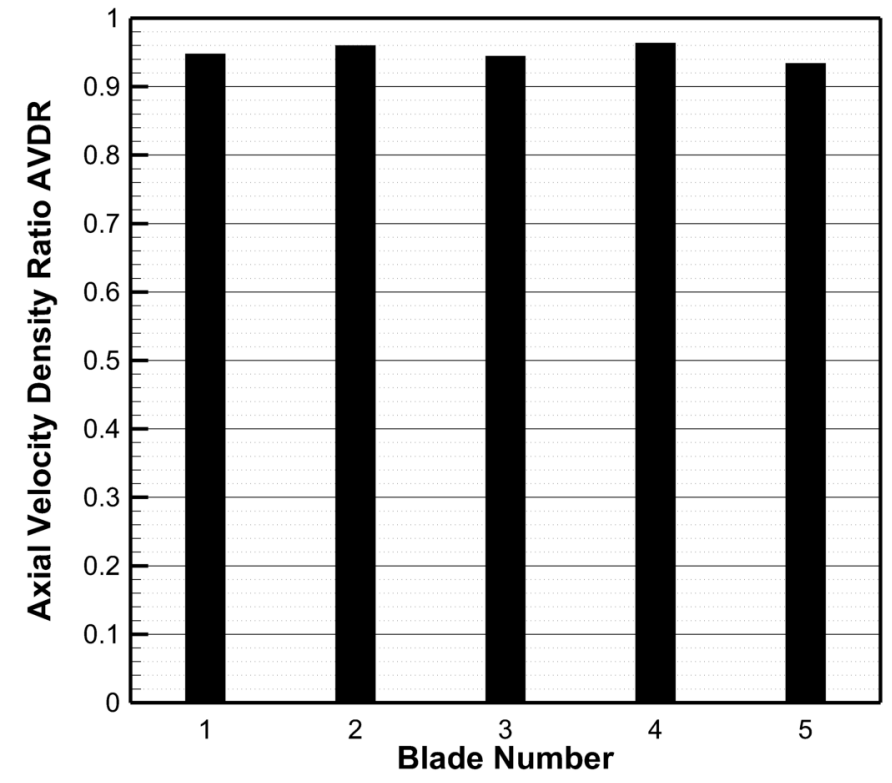

Figure 6.4: Periodicity of the Axial Velocity Density Ratio (AVDR) of the AF4 Cascade at $M_{2, i s}=1.2$

The AVDR values are comparable to values obtained in other cascades with similar flow acceleration. For example, Jeffries (2000) obtained values of AVDR in the range of 0.90 to 0.95 for a cascade named HS2, tested in the same wind tunnel.

\subsection{Test Cases}

The AF4 cascade was tested experimentally in the high-speed wind tunnel. These data were supplemented by experimental data previously collected by Corriveau (2005) for the HS1 family of cascades. In addition to the wind tunnel measurements, numerical simulations were carried out using boundary conditions taken from the wind tunnel data. The Mach number and Reynolds number ranges for the AF4 and HS1 cascades are provided in Figure 6.5.

The high-speed wind tunnel is controlled based on the downstream isentropic Mach number, which is obtained from the ratio of upstream total pressure to downstream static pressure. This control arrangement has the limitation that the Reynolds number increases concurrently with the Mach number. While it is important to keep this limitation in mind, it is not expected 


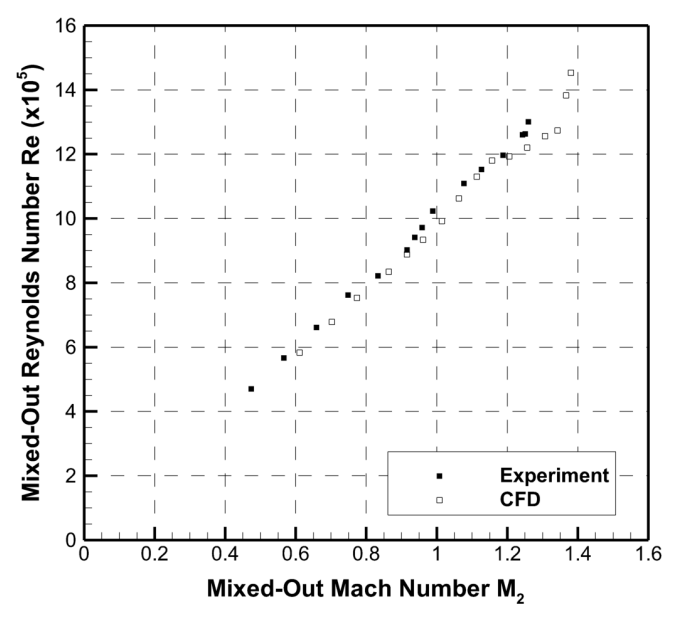

(a) AF4 Cascade

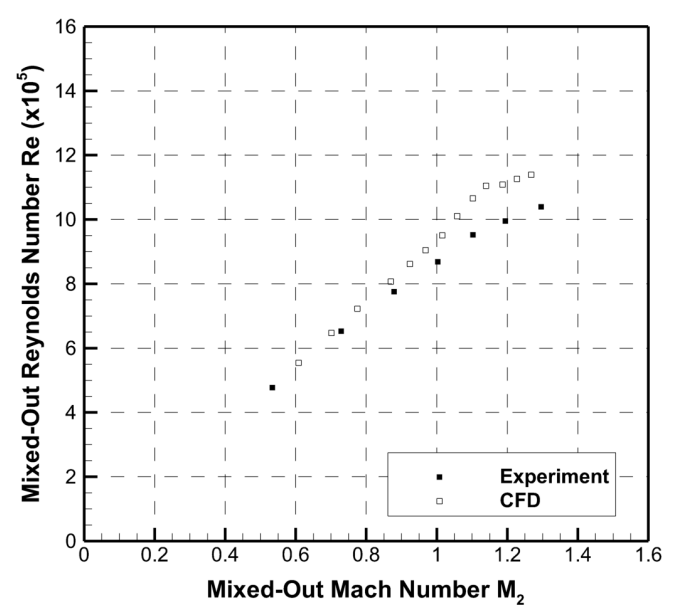

(c) HS1C Cascade

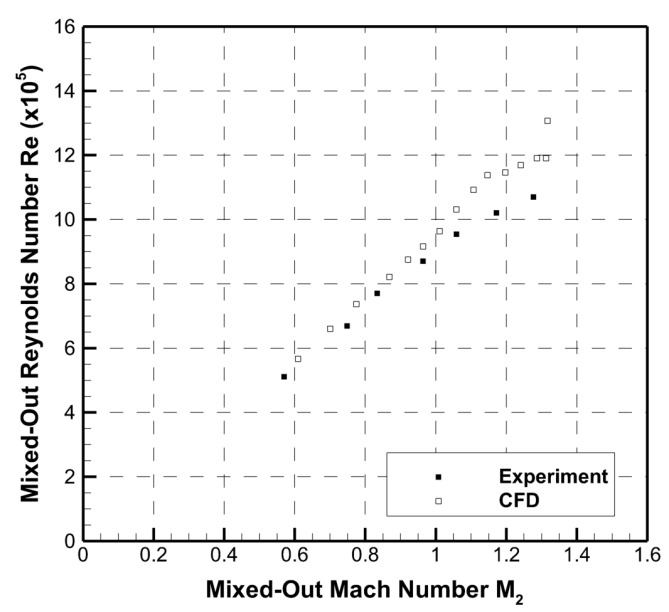

(b) HS1A Cascade

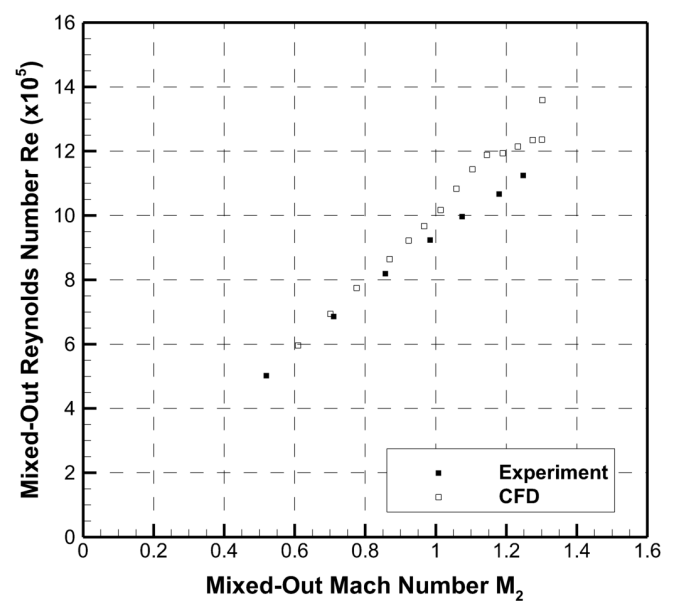

(d) HS1D Cascade

Figure 6.5: Mach Number and Reynolds Number Ranges of the Cascades Investigated in the Current Study 


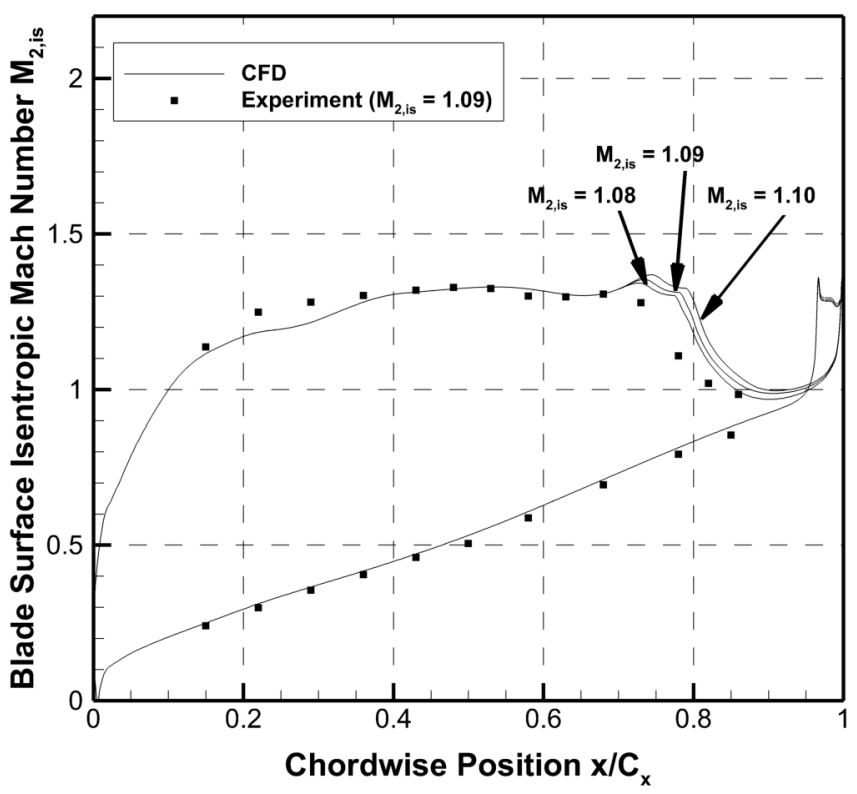

Figure 6.6: Comparison between Experimental and Numerical Blade Loadings for the AF4 Cascade

that the Reynolds number has a strong effect on the profile losses in the range of Reynolds numbers that occurs here. Based on an investigation by Corriveau $(2005)$ on the effect of the Reynolds number of the losses of the HS1 family of cascades, increasing the Reynolds number beyond about 750000 had only a moderate effect on the losses. Thus, the Reynolds number is expected to have only a moderate effect on the losses for runs at an outlet Mach number above roughly 0.8 .

\subsection{Blade Loading}

The blade loading is defined in this investigation based on the blade surface isentropic Mach number, as previously described in Section 4.4.2. The blade loadings are useful for comparing the CFD to the experimental results. One such comparison is made in Figure 6.6. showing the blade loading of the AF4 cascade. The upper line in Figure 6.6 represents the blade loading on the suction surface of the airfoil and the lower line represents the blade loading on the pressure surface. The experimental data are for an isentropic outlet Mach 
number of $M_{2, i s}=1.09$. An equivalent flow was generated numerically for the same outlet isentropic Mach number. Additional numerical simulations were performed at outlet isentropic Mach numbers slightly above and below the baseline case in order to observe the sensitivity of the loading distribution. The loading distribution obtained experimentally is reasonably well captured by the CFD. There is a mismatch in the loading distribution along the aft end of the blade suction surface where an abrupt flow deceleration occurs. As will be shown in Section 6.8, this flow deceleration results from the trailing edge shock from the adjacent blade impinging on the blade suction surface. The position of the shock impingement is highly dependent on the outlet Mach number of the flow, since the shock becomes more oblique as the Mach number is increased. The experimental and CFD cases may therefore have slightly different Mach number operating points, due to differences in the way the downstream isentropic Mach number is defined. Both the experiment and the CFD employ the upstream total pressure as a reference, but differ in the way that the downstream static pressure is obtained. In the CFD, the downstream static pressure is area-averaged across the measuring plane. In the experiment, a small manifold with four ports is connected to four endwall taps downstream of the cascade. This method was selected to provide a more robust measurement of static pressure in the case where an individual static tap is affected by a blade wake or a shock. The use of the manifold allows for more consistent control of the test section Mach number. However, it is possible for flow to occur within the tubes of the manifold as a result of pressure differences between ports, introduced by flow features or manufacturing defects in the drilled taps. The manifold arrangement thus causes ambiguity in the downstream static pressure measurement, and consequently in the downstream isentropic Mach number value. In hindsight, a more appropriate method of obtaining the downstream static pressure would have been to area-average the traverse measurements made by the static probe at the same operating conditions as the blade loading measurements. This method was already used to obtain the downstream static pressure in the mixed-out results, but the measurements were made at different wind tunnel operating points and could not be used with the blade loading data. 
The blade loadings for the four cascades are shown in Figure 6.7. Blade loadings were obtained at a large number of outlet isentropic Mach numbers for all four cascades, both experimentally and from the CFD. For Figure 6.7, a smaller selection of loading distributions were chosen in which the outlet isentropic Mach number was nearly the same between the experiment and the CFD. The mismatch in blade loading between the experiment and the CFD is less pronounced in the HS1 family of cascades than in the AF4 cascade, though it is unclear how the downstream Mach number was defined for the experimental blade loadings obtained from Corriveau (2005).

The loading distribution of a turbine blade provides insight into the regions of high flow acceleration and deceleration, and equivalently the regions of favourable and adverse pressure gradients. The blade loading for the AF4 cascade is shown in Figure 6.7a. Recalling the blade curvature distributions shown in Figure 3.11, the strong curvature near the front of the AF4 blade causes strong flow acceleration near the leading edge. Even at the low outlet isentropic Mach number of 0.79 , a pocket of supersonic flow occurs from the axial positions of $x / C_{x}=0.1$ to just below $x / C_{x}=0.5$. This pocket of supersonic flow ends in a strong shock, represented by the strong adverse pressure gradient.

The blade passage becomes choked at an outlet isentropic Mach number of roughly 0.88 , which is not shown in Figure 6.7a. The choked condition is confirmed by the fact that the pressure side blade loading and the front portion of the suction side blade loading become fixed at outlet isentropic Mach numbers above 0.88 .

At supersonic outlet Mach numbers, the flow accelerates at roughly $x / C_{x}=0.67$, then undergoes an abrupt deceleration, for example, at $x / C_{x}=0.75$ for $M_{2}=1.12$. The acceleration occurs because the blades form a diverging flow path downstream of the throat, allowing the flow to continue accelerating supersonically. To a lesser extent, the acceleration can also be attributed to the expansion waves that are generated from flow around the trailing edge of the adjacent blade, as shown in Figure 6.8. These waves propagate across the blade passage to accelerate the flow along the suction surface. Slightly downstream of these expansion waves, the trailing edge shock forms on the adjacent blade and impinges on the suction surface boundary layer. A small separation bubble occurs at the point of the shock-boundary layer interaction 


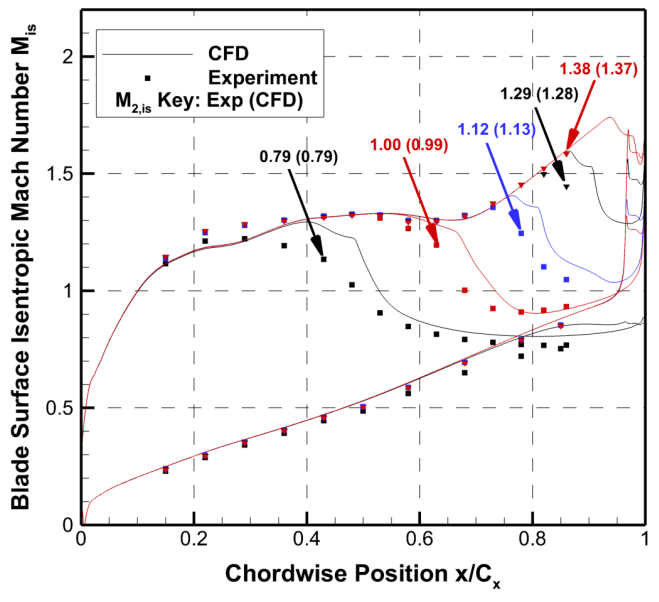

(a) AF4 Cascade

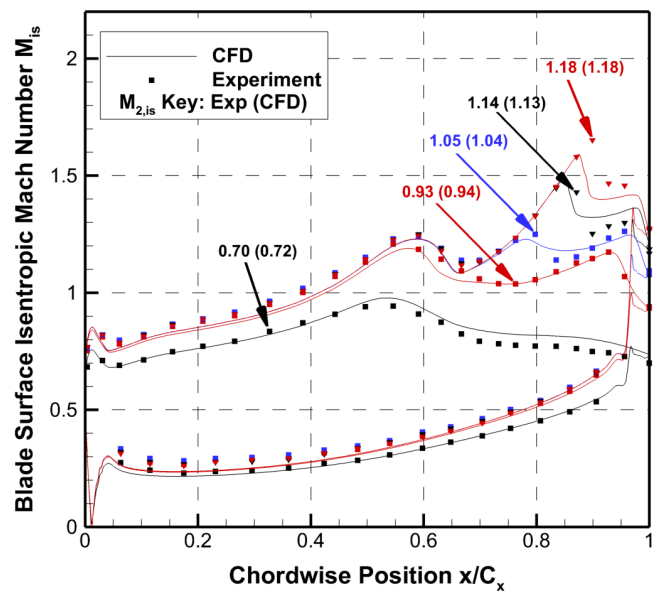

(c) HS1C Cascade

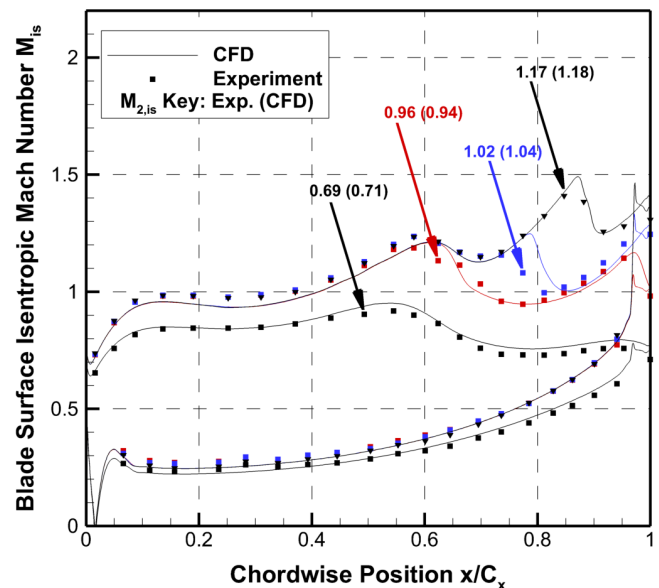

(b) HS1A Cascade

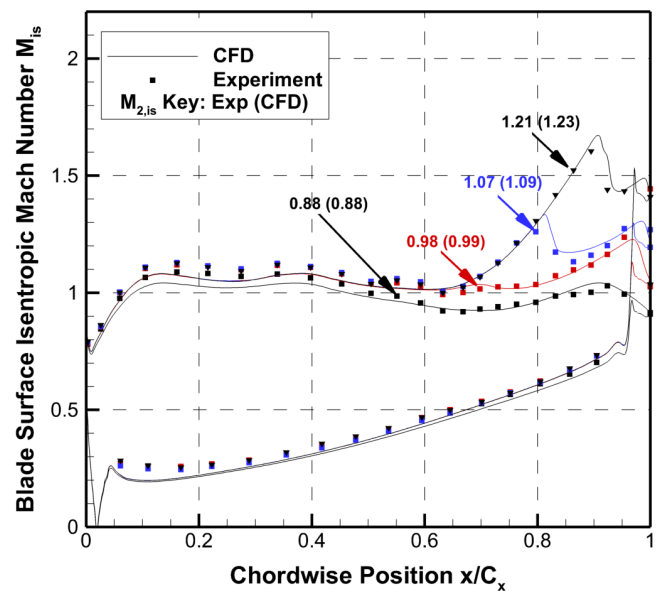

(d) HS1D Cascade

Figure 6.7: Blade Loading, Expressed in Terms of the Blade Surface Isentropic Mach Number 


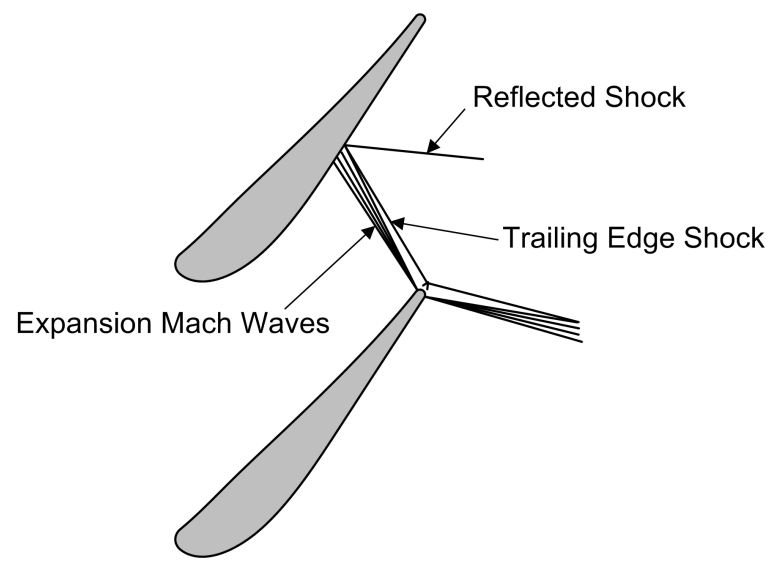

Figure 6.8: Schematic of Trailing Edge Expansion Waves and Shocks, Which Propagate across the Flow Passage

as a result of the adverse pressure gradient caused by the shock. However, the boundary layer reattaches after this interaction and remains attached up to the trailing edge, as evidenced by the low profile losses which will be presented in the next section. The acceleration and deceleration caused by the supersonic flow shown in Figure 6.8 is also present in the three HS1 cascades.

Downstream of the deceleration caused by the shock-boundary layer interaction, the pressure on the AF4 suction surface remains roughly constant. This behaviour is caused by the low wall curvature near the aft end of the AF4 blades, therefore causing little flow acceleration.

The blade loading for the HS1A cascade is shown in Figure 6.7b, This blade is considered mid-loaded. A suction peak occurs at an axial position of roughly $x / C_{x}=0.5$, followed by a strong adverse pressure gradient. At subsonic outlet isentropic Mach numbers, the flow deceleration is followed by a long region of nearly constant pressure, which would typically indicate flow separation. However, it will be seen in Section 6.8 that no flow separation occurs.

The HS1C cascade was designed by Corriveau (2005) to have an aft-loaded pressure distribution. This objective was achieved through lower suction side flow velocities on the front half of the blade, as shown in Figure 6.7c.

The HS1D cascade was intended to have a front-loaded pressure distribution by Corriveau (2005). As shown in Figure 6.7d, even at an outlet isentropic Mach number of 0.88, a long 
region of supersonic flow occurs over the front end of the suction surface. An adverse pressure gradient, though weak, exists over about $50 \%$ of the blade chord.

\subsection{Profile Losses}

The profile losses are expressed as a total pressure loss coefficient $(Y)$. Values for this coefficient are obtained by taking wake traverse data at the blade midspan and applying the mixing-out procedures described in Section 4.4 in order to obtain the corresponding fully mixed-out flow quantities. The effect of Mach number on the profile losses is shown in Figure 6.9 for the four cascades. The figures show both the experimental and numerical results. All numerical results were generated in the current study. The experimental results for the AF4 cascade were also collected in the current study, while the experimental results for the HS1 cascades were collected by Corriveau 2005.

An important feature in Figure 6.9 is the loss "plateau". The term loss plateau is used in this study to describe the following trend in profile loss coefficient. As the mixed-out Mach number reaches high subsonic values, the profile loss coefficient begins to increase. At transonic conditions, the coefficient increases less, and sometimes even decreases with increasing mixed-out Mach number, representing the lower limit of the loss plateau. The upper limit occurs at a supersonic mixed-out Mach number, in which case the profile loss coefficient begins to rise significantly with Mach number. This loss behaviour can be compared to the midspan loss predictions made using the Kacker \& Okapuu (1982) loss correlation, seen previously in Figure 2.21. The loss plateau is not captured by the correlation.

The four cascades exhibit different extents of this loss plateau. The most noticeable case is that of the AF4 cascade, which shows a decrease in profile losses as the mixed-out Mach number increases beyond about 0.96. The most moderate loss plateau occurs in the HS1C cascade, shown in Figure 6.9c. The experimental results from Corriveau (2005) show a narrow loss plateau, but this trend depends on a single data point at $M_{2}=1.1$. The numerical results for HS1C do not exhibit a supersonic loss plateau. The numerical simulations performed by Corriveau (2005) also show a smooth rise in losses. Corriveau attributed the mismatch 


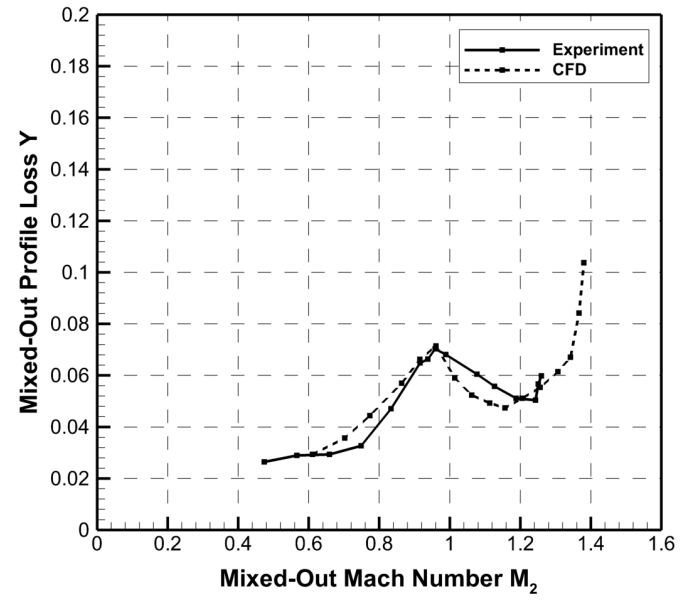

(a) AF4 Cascade

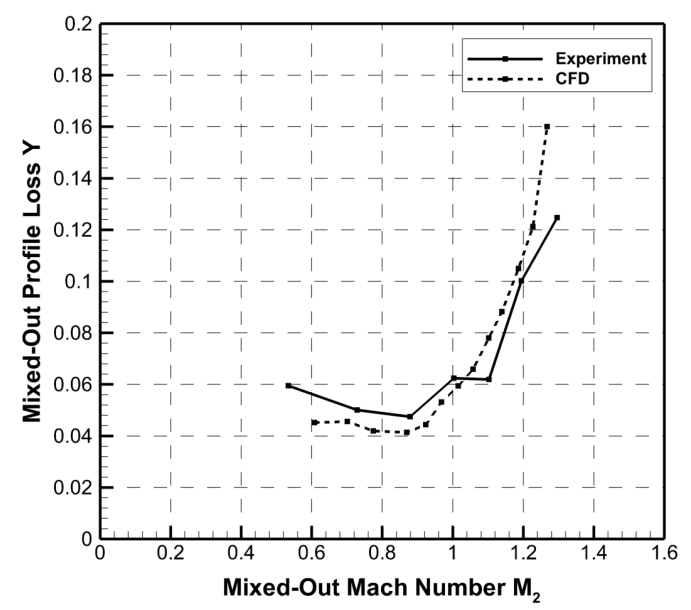

(c) HS1C Cascade

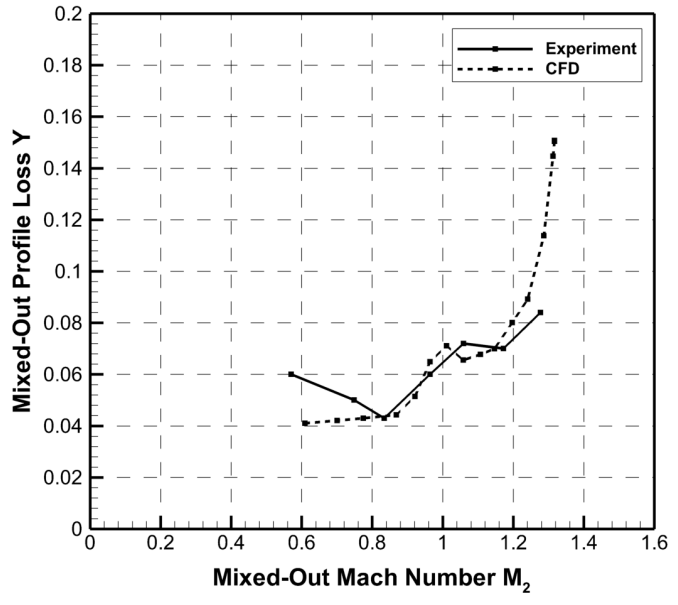

(b) HS1A Cascade

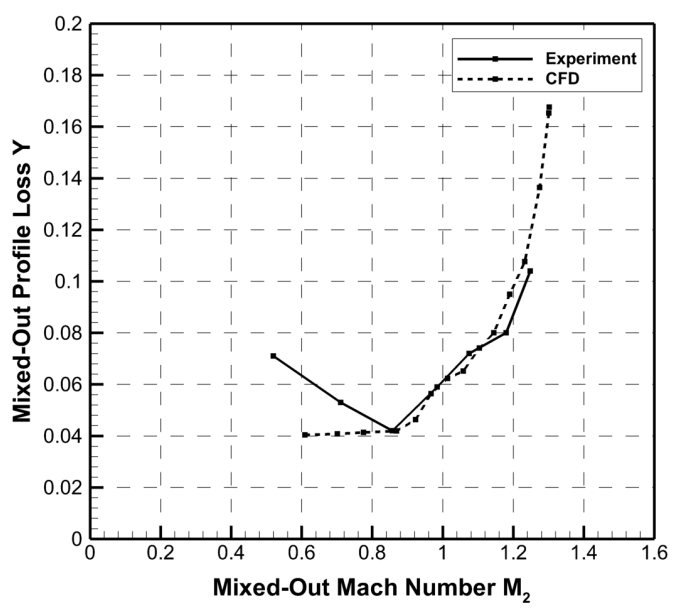

(d) HS1D Cascade

Figure 6.9: Effect of Mach Number on the Total Pressure Loss Coefficient 
between the experimental and numerical results to the absence of a transition model in the numerical simulations. Since the gamma-theta transition model was used in the current study and a loss plateau was still not observed in the numerical results, it is possible that the loss plateau observed in the experimental results may simply be the consequence of one erroneous data point at $M_{2}=1.1$.

Comparing the experimental and numerical results, the most notable discrepancy occurs at Mach numbers below $M_{2}=0.9$, particularly for the HS1A and HS1D cascades. Intuition would suggest that the mismatch is a Reynolds number effect, since the Reynolds number becomes more influential on the profile losses in the ranges encountered at low outlet Mach numbers. However, as seen in Section 6.3, the Reynolds numbers show reasonable agreement between the experiment and the CFD. The discrepancy may tentatively be attributed to the

recent improvements made to the wind tunnel Mach number control system, by Hall (2012). Prior to the improvements, the Mach number in the wind tunnel was rarely operated below outlet Mach numbers of 0.55 , due to concerns about the accuracy of the Mach number.

\subsection{Outlet Flow Angle}

The outlet flow angles measured in the high-speed wind tunnel and predicted by the simulations are included for completeness, as shown in Figure 6.10. The experimental trends are broadly reflected in the CFD, but the CFD seems to generally underpredict the outlet flow angle, particularly at subsonic mixed-out Mach numbers.

It is expected that the outlet flow angle decreases when the Mach number is increased beyond sonic conditions. Downstream of the throat, the streamtubes must expand in order to reach supersonic Mach numbers. This expansion is accomplished by decreasing the flow angle towards the axial direction, allowing the cross-sectional area observed by the streamtubes to increase. 


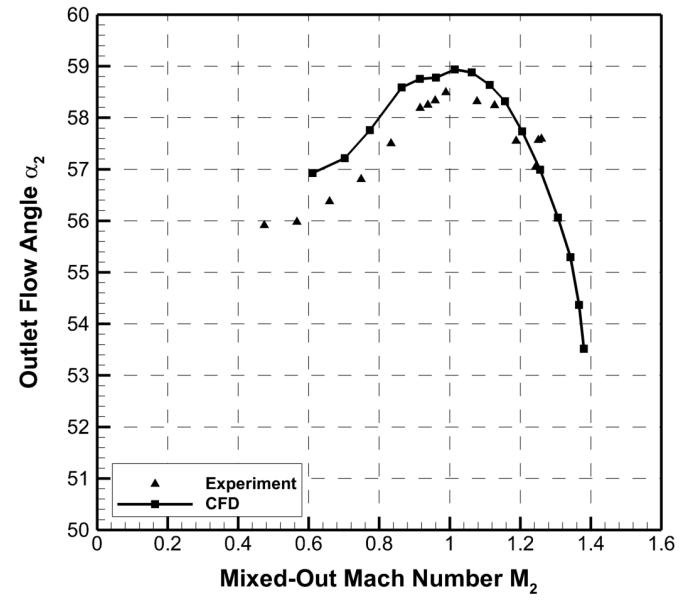

(a) AF4 Cascade

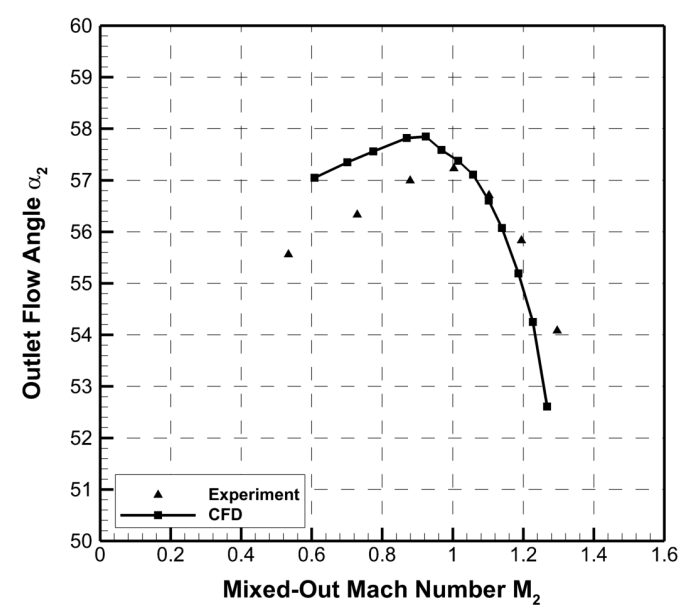

(c) HS1C Cascade

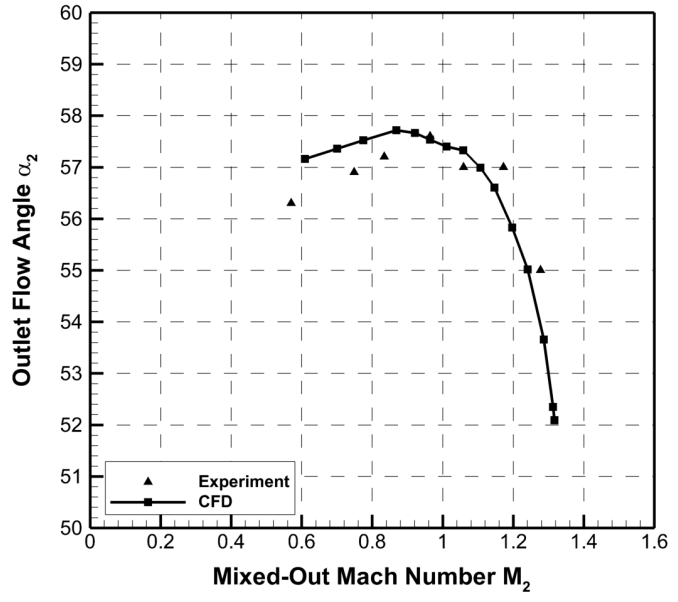

(b) HS1A Cascade

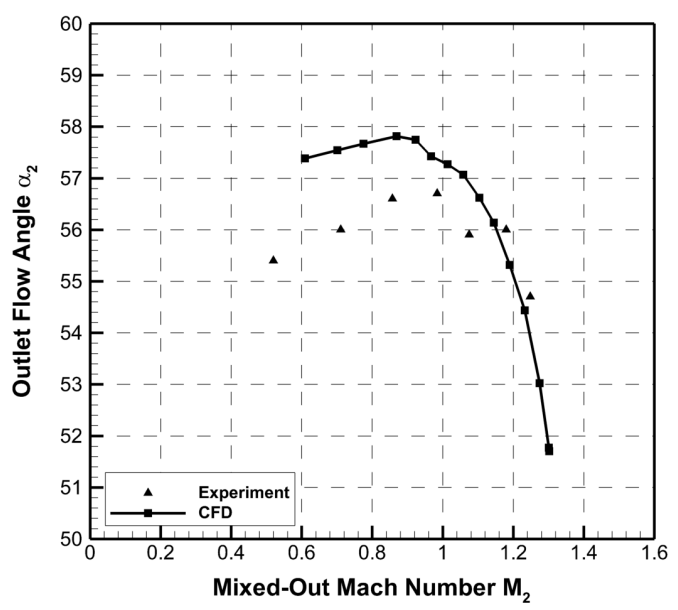

(d) HS1D Cascade

Figure 6.10: Effect of Mach Number on the Outlet Flow Angle 


\subsection{Base Pressure}

As described in Section 2.5.2, the base pressure is the static pressure measured at the trailing edge surface, which faces the region of separated flow immediately downstream of the trailing edge. The static pressure distribution along the surface of the trailing edge, extracted from the numerical simulations, is shown for the AF4 cascade in Figure 6.11, and is representative of all four cascades. The distribution is shown as a function of the angle normal to the blade surface, with $0^{\circ}$ corresponding to the direction of the outlet metal angle. Figure 6.11 strongly resembles the measured distribution presented by Sieverding et al. (1979). The wide region of uniform pressure is caused by the separated flow downstream of the trailing edge, known as the base region. The narrow pressure drops on either side of the base region result from the expanding flow just upstream of the separation shocks.

The base pressure was measured by Corriveau (2005) using a static tap at the trailing edge of one blade in each of the HS1 cascades. The tap diameter was $20 \%$ of the trailing edge thickness and was aligned with the outlet metal angle. From Figure 6.11, at all Mach numbers simulated, the region of approximately constant pressure spans well over $20 \%$ of the trailing edge thickness. Thus it seems likely that the base pressure measurements made by Corriveau are representative of the actual pressure in the base region, despite the relatively large size of the tap.

In the current study, the base pressure is provided in the form of the base pressure coefficient, defined as follows:

$$
C_{b}=\frac{P_{b}-P_{2}}{P_{o 2}-P_{2}}
$$

where $P_{b}$ is the base pressure, and $P_{o 2}$ and $P_{2}$ are the mixed-out total pressure and static pressure, respectively. The denominator $\left(P_{o 2}-P_{2}\right)$ reduces to the dynamic pressure $\left(q_{2}\right)$ in the case of incompressible flow.

The effect of the outlet mixed-out Mach number on the base pressure coefficient is shown in Figures 6.12a to 6.12d for the AF4, HS1A, HS1C and HS1D cascades, respectively. The AF4 cascade was not instrumented with a base pressure tap at the trailing edge, so only CFD 

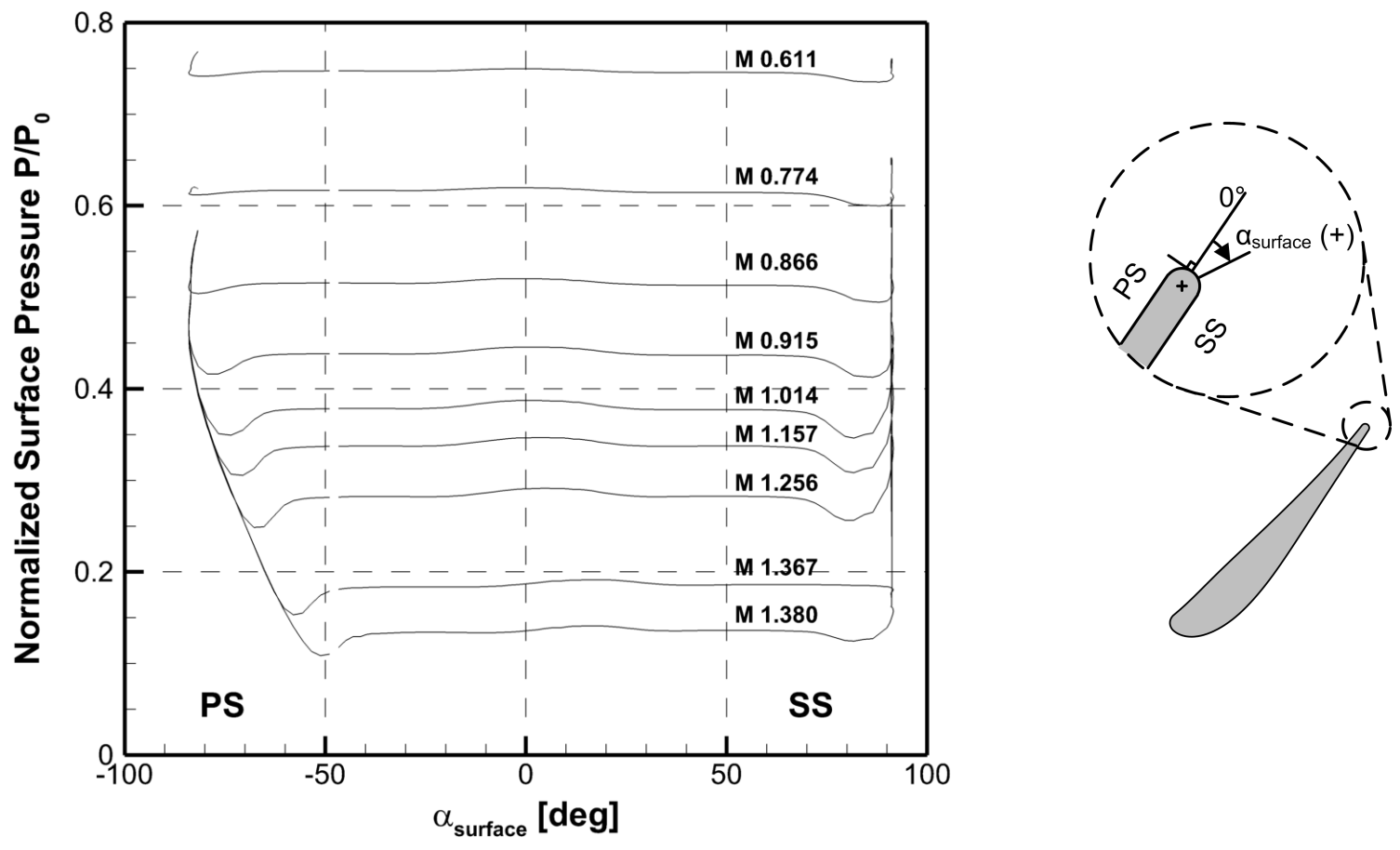

Figure 6.11: Base Pressure Distribution around the Trailing Edge Surface of the AF4 cascade as a Function of the Angle of the Blade Surface Normal Vector $\alpha_{\text {surface }}$ 
data are available. While the CFD and experiment do not agree perfectly, the qualitative trends are captured by the CFD simulations.

The trends in variation of base pressure coefficient with the outlet Mach number are very different for the four cascades. However, some general trends are observed. The base pressure coefficients exhibit a moderate to large decrease in the vicinity of a mixed-out Mach number of 1.0. The base pressure coefficients also appear to decrease significantly at supersonic Mach numbers. The only exception is the HS1A cascade experiment, but the final decrease in base pressure coefficient would likely have been observed if data had been collected at higher mixed-out Mach numbers.

In order to provide context to these data, the base pressure values are compared to the Sieverding et al. (1979) base pressure correlation. Sieverding et al. present the base pressure correlation on a plot of $P_{b} / P_{o 1}$ against $P_{2} / P_{o 1}$. The latter ratio is equivalent to the outlet isentropic Mach number. The trend lines proposed in the correlation are a function of the following parameter:

$$
\Phi=\frac{W_{e}+\theta_{u}}{2}
$$

where $W_{e}$ is the trailing edge wedge angle and $\theta_{u}$ is the unguided turning angle (both defined schematically in Figure 6.13). The values of these parameters for the four cascades in this study are summarized in Table 6.1. Unfortunately, Sieverding et al. (1979) did not provide a physical explanation for correlating based on the trailing edge wedge angle and the unguided turning angle.

The base pressures in the four cascades in this study are compared with the Sieverding et al. (1979) base pressure correlation in Figures 6.14 to 6.17. The dashed diagonal line represents the case where the base pressure is equal to the downstream mixed-out static pressure. Thus, data points located below the dashed diagonal line indicate that the base pressure is lower than the downstream mixed-out static pressure, meaning that there is suction in the base region.

In order to summarize the base pressure behaviour shown in Figures 6.14 to 6.17, the cascades with the lowest and highest values of $\Phi$ will be discussed first (AF4, 4.34 ${ }^{\circ}$; and 


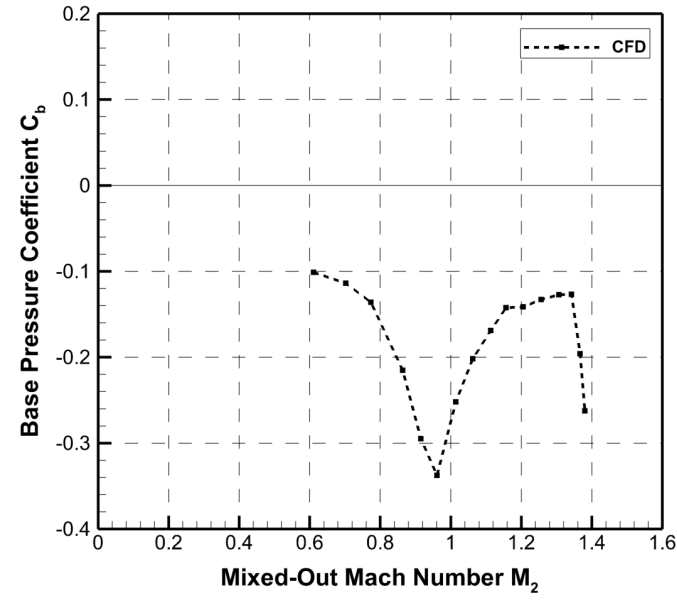

(a) AF4 Cascade

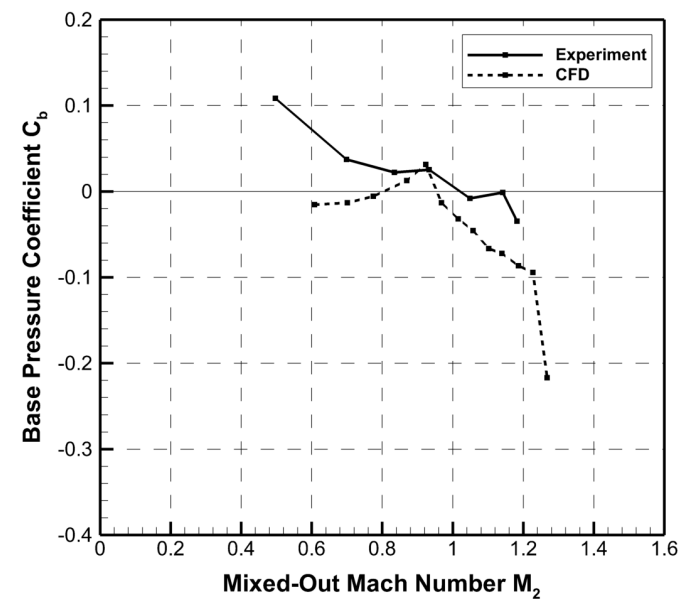

(c) HS1C Cascade

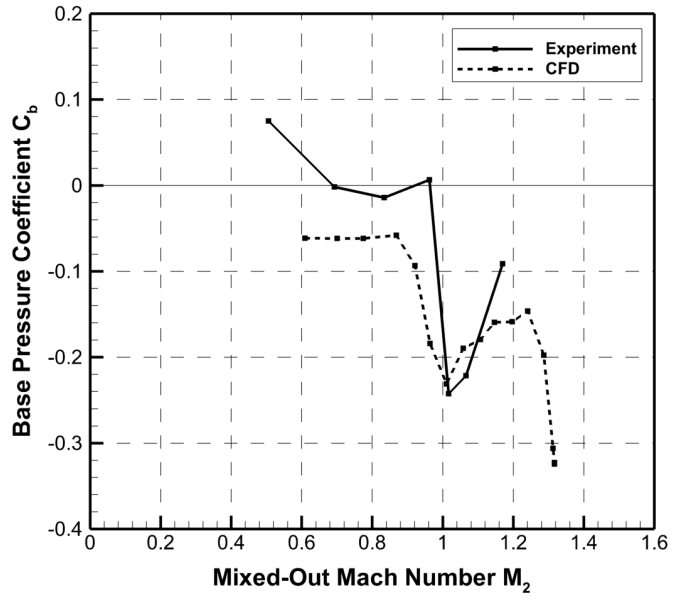

(b) HS1A Cascade

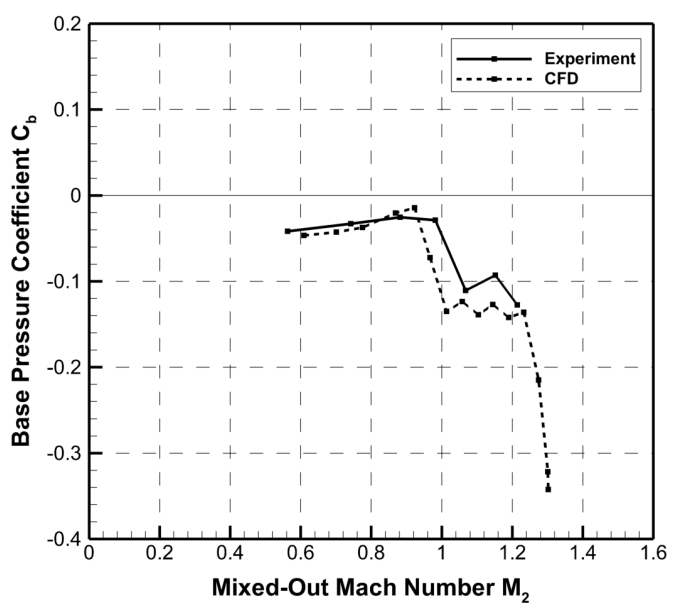

(d) HS1D Cascade

Figure 6.12: Base Pressure Coefficient $\left(C_{b}\right)$ Variation with Mixed-Out Mach Number 
Table 6.1: Summary of Geometry Values Used in the Sieverding et al. (1979) Base Pressure Correlation

\begin{tabular}{llcccc}
\hline Symbol & Parameter & AF4 & HS1A & HS1C & HS1D \\
\hline$W e_{T E}$ & TE Wedge Angle & $5^{\circ}$ & $6^{\circ}$ & $6^{\circ}$ & $6^{\circ}$ \\
$\theta_{u}$ & Unguided Turning & $3.68^{\circ}$ & $11.5^{\circ}$ & $14.5^{\circ}$ & $11.5^{\circ}$ \\
$\Phi$ & Sieverding et al. (1979) & $4.34^{\circ}$ & $8.75^{\circ}$ & $10.25^{\circ}$ & $8.75^{\circ}$ \\
& Parameter & & & & \\
\hline
\end{tabular}

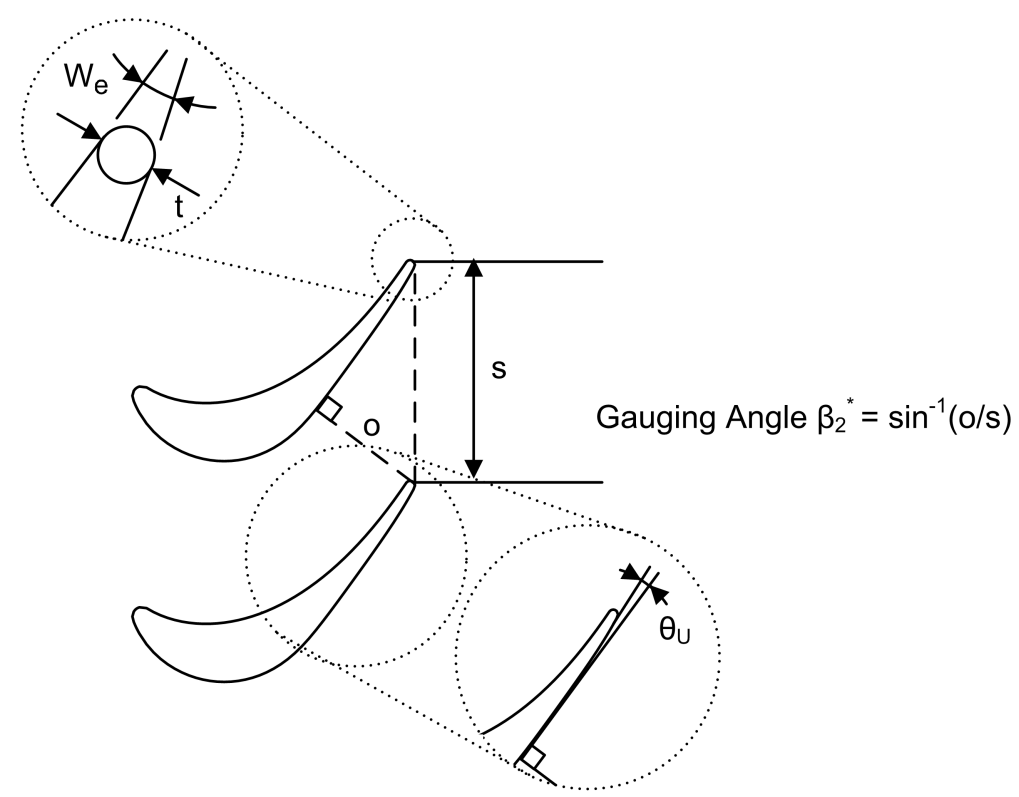

Figure 6.13: Definitions of the Symbols Used in the Sieverding et al. (1979) Base Pressure Correlation

HS1C, $10.25^{\circ}$ ). The AF4 cascade is shown in Figure 6.14. Beginning with the top right-hand corner, which corresponds to a low outlet Mach number, the base pressure is slightly below the downstream mixed-out static pressure. Moving left (increasing the outlet Mach number), the base pressure drops well below the downstream static pressure near an outlet Mach number of 1.0. Moving further left, the base pressure returns to a value slightly below the downstream static pressure. The behaviour just described agrees with the behaviour predicted by the Sieverding et al. (1979) correlation for low values of $\Phi$. At the highest Mach numbers tested, the base pressure decreases abruptly. It will be seen in Section 6.8 that this decrease occurs when the trailing edge shocks of the adjacent blade become sufficiently oblique to impinge on 


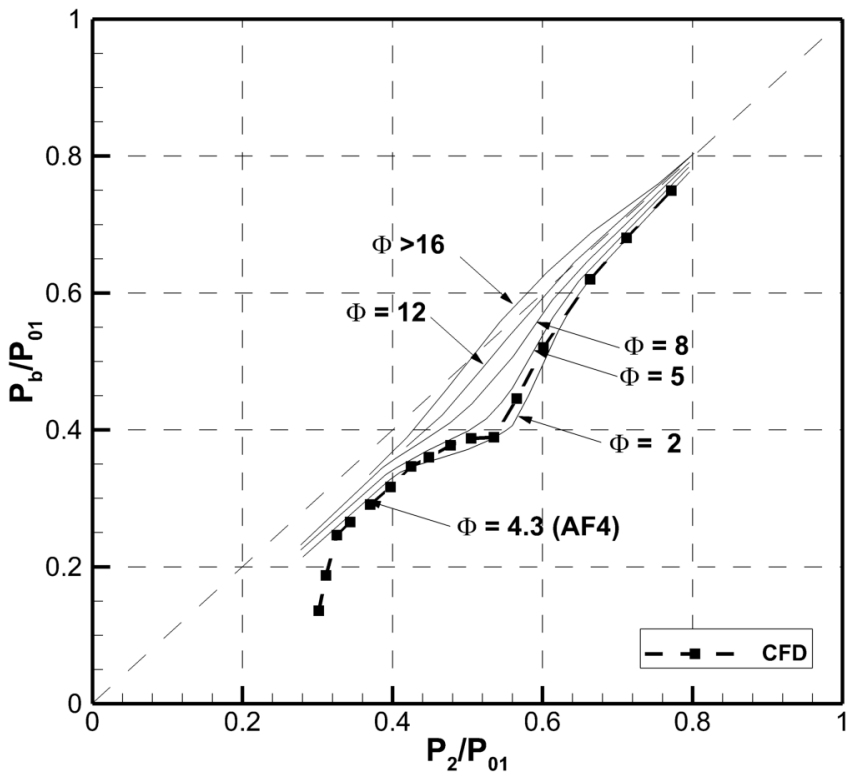

Figure 6.14: Comparison of the Base Pressure Measurements of the AF4 Cascade with the Sieverding et al. (1979) Correlation

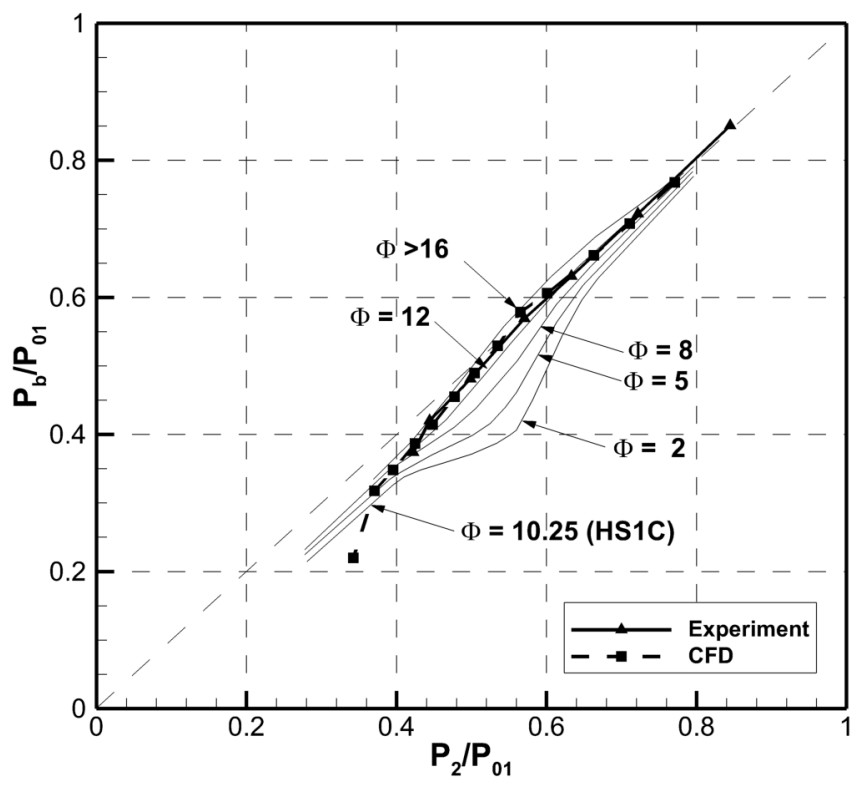

Figure 6.15: Comparison of the Base Pressure Measurements of the HS1C Cascade with the Sieverding et al. (1979) Correlation 


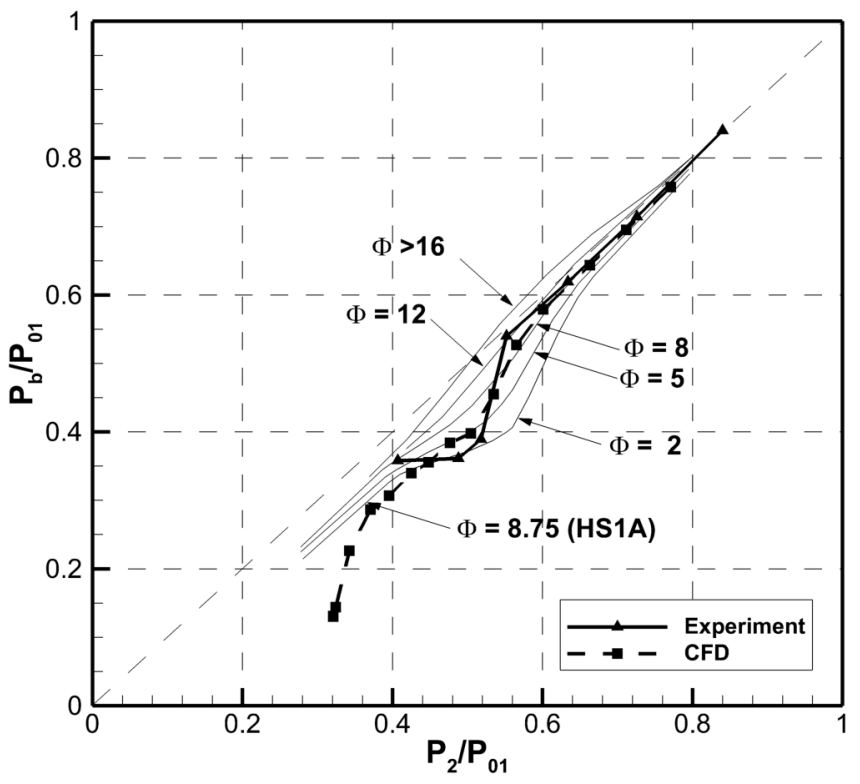

Figure 6.16: Comparison of the Base Pressure Measurements of the HS1A Cascade with the Sieverding et al. (1979) Correlation

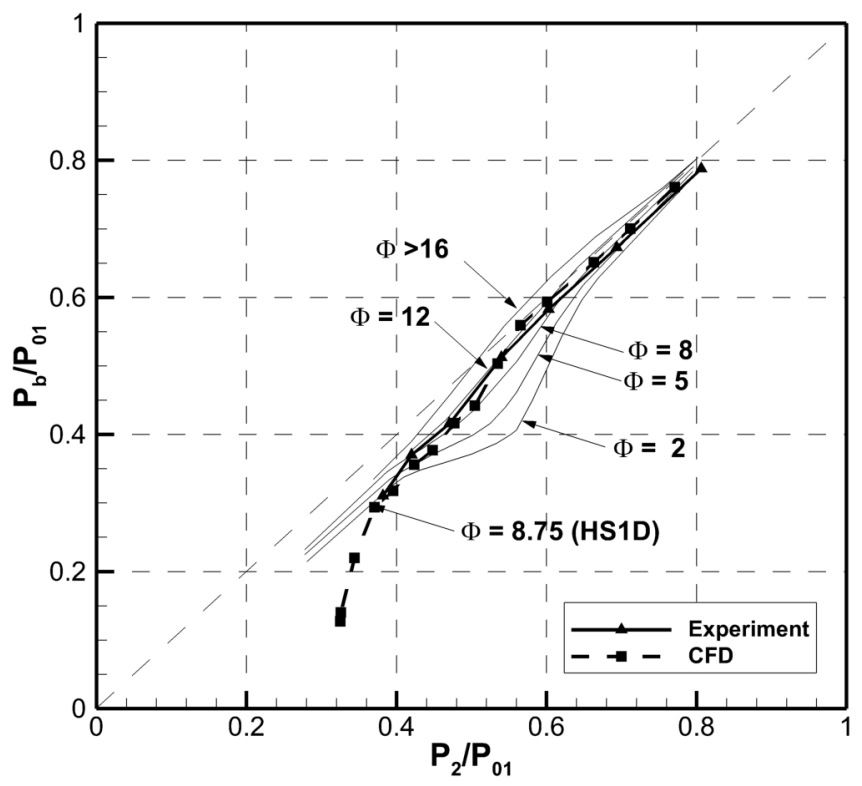

Figure 6.17: Comparison of the Base Pressure Measurements of the HS1D Cascade with the Sieverding et al. (1979) Correlation 
the base region. The base pressure is strongly affected by this interaction, and the Sieverding et al. (1979) correlation makes no attempt to capture the base pressure in this condition. According to Sieverding et al. (1979), the base pressure in this case is primarily influenced by the gauging angle $\beta_{2}^{*}$, a parameter defined in Figure 6.13. In order for the trailing edge shock to interact with the base region of an adjacent blade, the angle of this shock must roughly correspond to the gauging angle $\left(\beta_{2}^{*}\right)$. Thus, blades with different gauging angles reach this condition at different Mach numbers.

Unlike the AF4 cascade, which exhibits strong variations of base pressure as the outlet Mach number is increased, the base pressure of the HS1C cascade remains much closer to the downstream mixed-out static pressure $\left(P_{2}\right)$. The agreement of the HS1C cascade with the Sieverding et al. correlation, for a parameter value of $10.25^{\circ}$, is very good.

The Sieverding et al. (1979) correlation gives the broad trends in base pressure behaviour, but comparing the HS1A and HS1D cascades shows that the $\Phi$ parameter does not fully capture the behaviour. The HS1A and HS1D cascades have the same value of $\Phi\left(8.75^{\circ}\right)$, but do not show the same behaviour, as shown in Figures 6.16 and 6.17. Specifically, the HS1A cascade exhibits a stronger decrease in base pressure at sonic outlet conditions than the HS1D cascade. While both airfoils have the same unguided turning angle and trailing edge wedge angle, referring back to the blade curvature distributions in Figure 3.11 the blade curvature on the suction surface is distributed differently between the throat and the trailing edge. The possible reasons why this would affect the base pressure will be discussed in Section 6.9.

\subsection{Wake Profiles and Blade-to-Blade Flow Features}

The midspan blade-to-blade flow features are presented through a series of contour plots of the Mach number, as obtained from the numerical simulations, accompanied by the previously presented plots of profile loss and base pressure. Overlaid on each contour plot is the wake profile across one blade pitch. The wake profile is extracted at the same distance downstream of the trailing edge as the wake measurement plane in the high-speed wind tunnel $\left(0.5 C_{x}\right.$ downstream of the cascade trailing edge). The wake profile is provided in the form of a local 
total pressure loss coefficient $\left(Y_{\text {local }}\right)$, which is defined as follows:

$$
Y_{\text {local }}=\frac{P_{o 1}-P_{o}}{P_{o 2}-P_{2}}
$$

where $P_{o 1}$ is the upstream total pressure, $P_{o}$ is the local value of total pressure, and $P_{o 2}$ and $P_{2}$ are the mixed-out total pressure and static pressure, respectively. The wake profile is useful for identifying areas of high loss production in the wake.

Every contour plot of the Mach number shows the flow features at a particular mixed-out Mach number $\left(M_{2}\right)$. Above each contour plot, the profile loss and base pressure coefficient plots (from Sections 6.5 and 6.7) are included in order to relate the flow behaviour back to the previously presented results. A vertical line across these plots indicates the Mach number of the current contour plot.

Figures 6.18 to 6.23 show the flow features of the AF4 cascade. Figures 6.24 to 6.28 correspond to the HS1A cascade. Figures 6.29 to 6.32 correspond to the HS1C cascade. Figures 6.33 to 6.36 correspond to the HS1D cascade. In total, this section contains about twenty full-page contour plots. Though these plots add length to the text, it was considered that reducing the image size would negatively impact the readability. The most representative individual cases were selected, but the contour plots are most valuable when viewed as a group in sequence, thus showing the evolution of flow features as the Mach number is increased.

From Figures 6.18 and 6.19, showing Mach number contour plots for the AF4 cascade, the smaller peak in profile losses at an outlet Mach number of 0.96 is associated with the appearance of the trailing edge shock system. Comparing the wake profiles in Figures 6.18 and 6.19 , there is an increase in losses in the trailing edge wake. There is also a region of increased losses outside of the trailing edge wake which appears to originate from the trailing edge shocks. The base pressure coefficient decreases as the profile losses increase, and likely has an influence on the wake losses and the shock losses. Firstly, a low base pressure tends to increase losses in the wake by applying a pressure force between the fluid and the trailing edge surface. This pressure force can be considered as a "drag" force on the blade. Secondly, as the suction and pressure side flows pass through the sudden increase in area at the trailing 

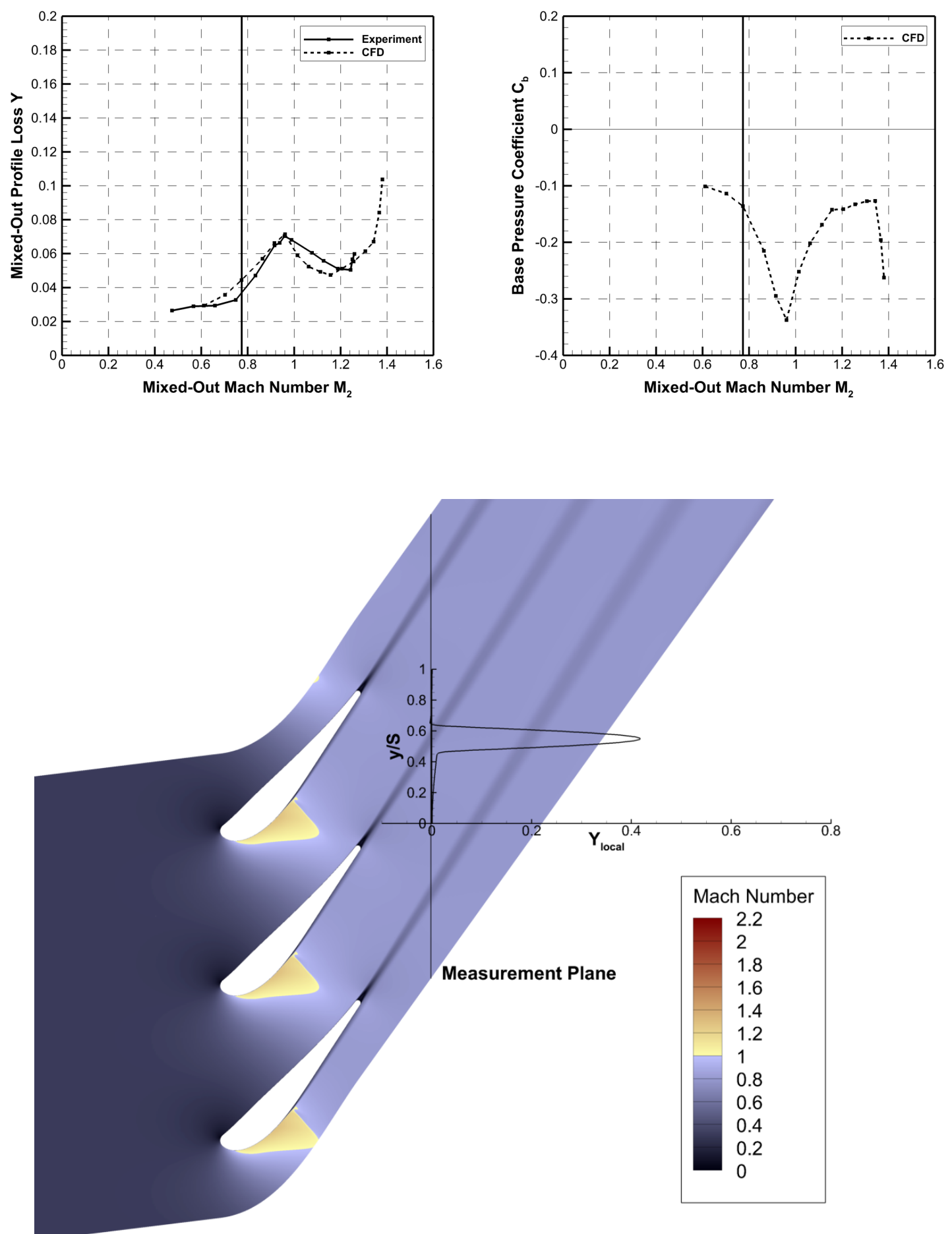

Figure 6.18: Blade-to-Blade Flow Features and Wake Profile of the AF4 Cascade at a Mixed-Out Mach Number of 0.77 (Profile Losses and Base Pressure Inset) 

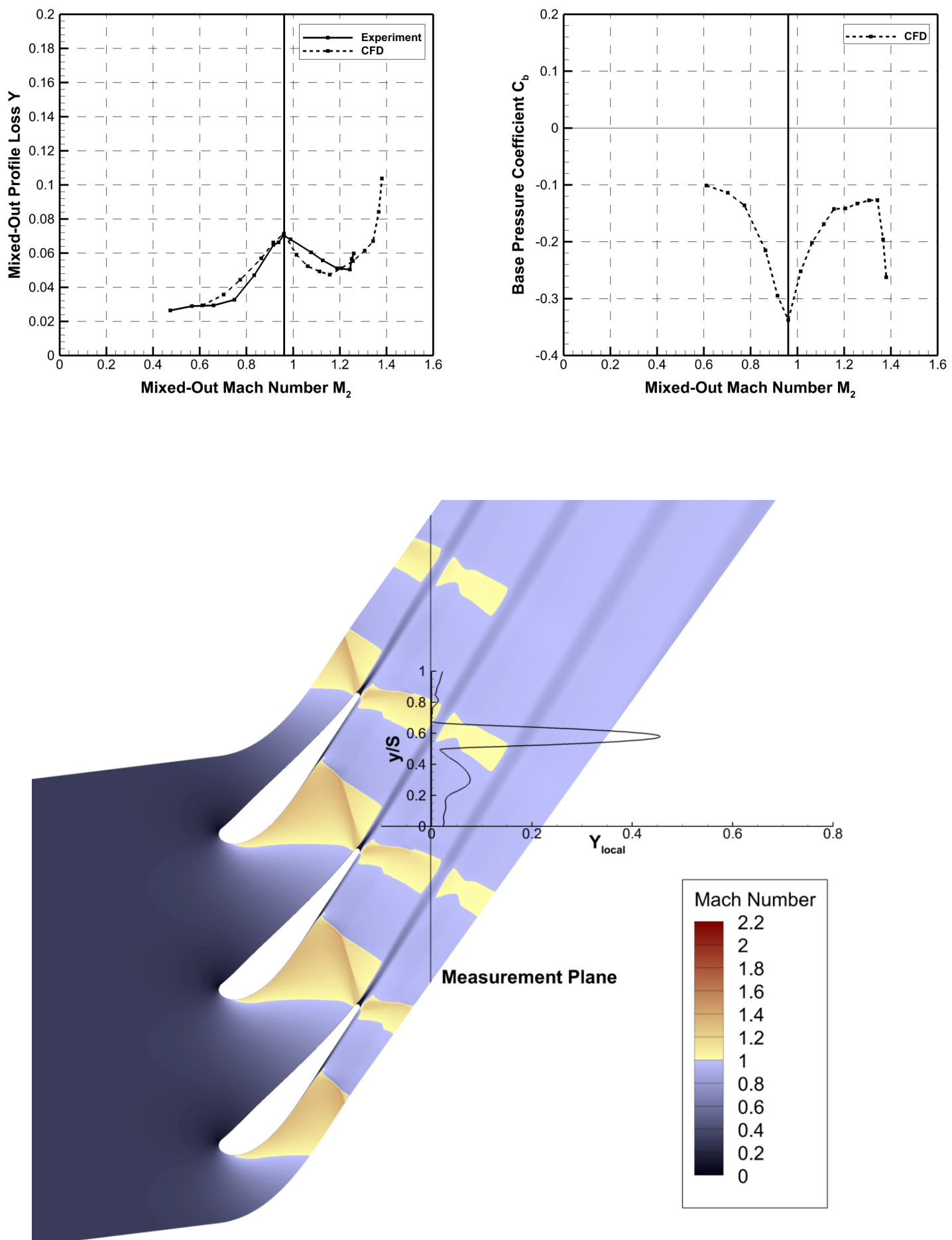

Figure 6.19: Blade-to-Blade Flow Features and Wake Profile of the AF4 Cascade at a Mixed-Out Mach Number of 0.96 (Profile Losses and Base Pressure Inset) 

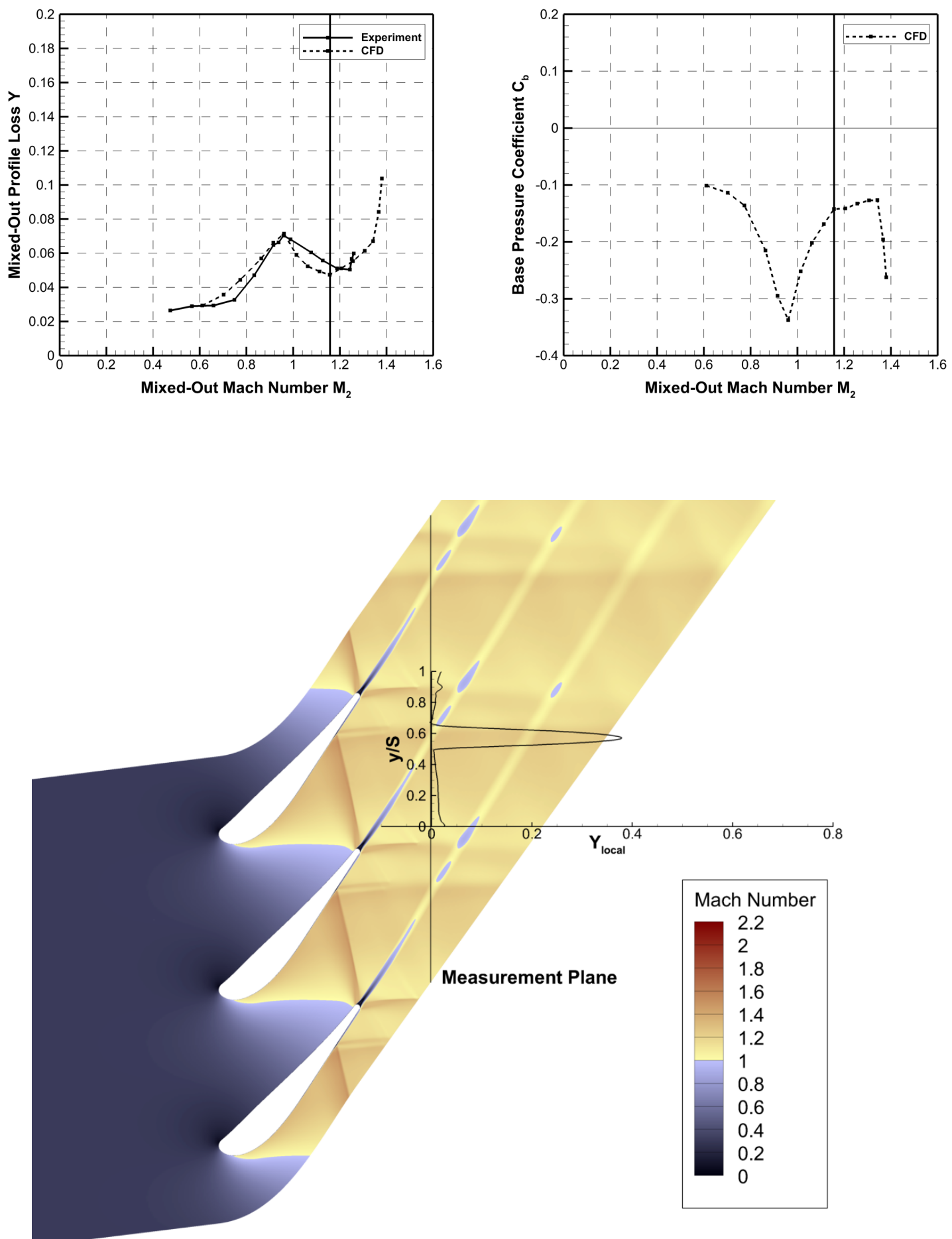

Figure 6.20: Blade-to-Blade Flow Features and Wake Profile of the AF4 Cascade at a Mixed-Out Mach Number of 1.16 (Profile Losses and Base Pressure Inset) 

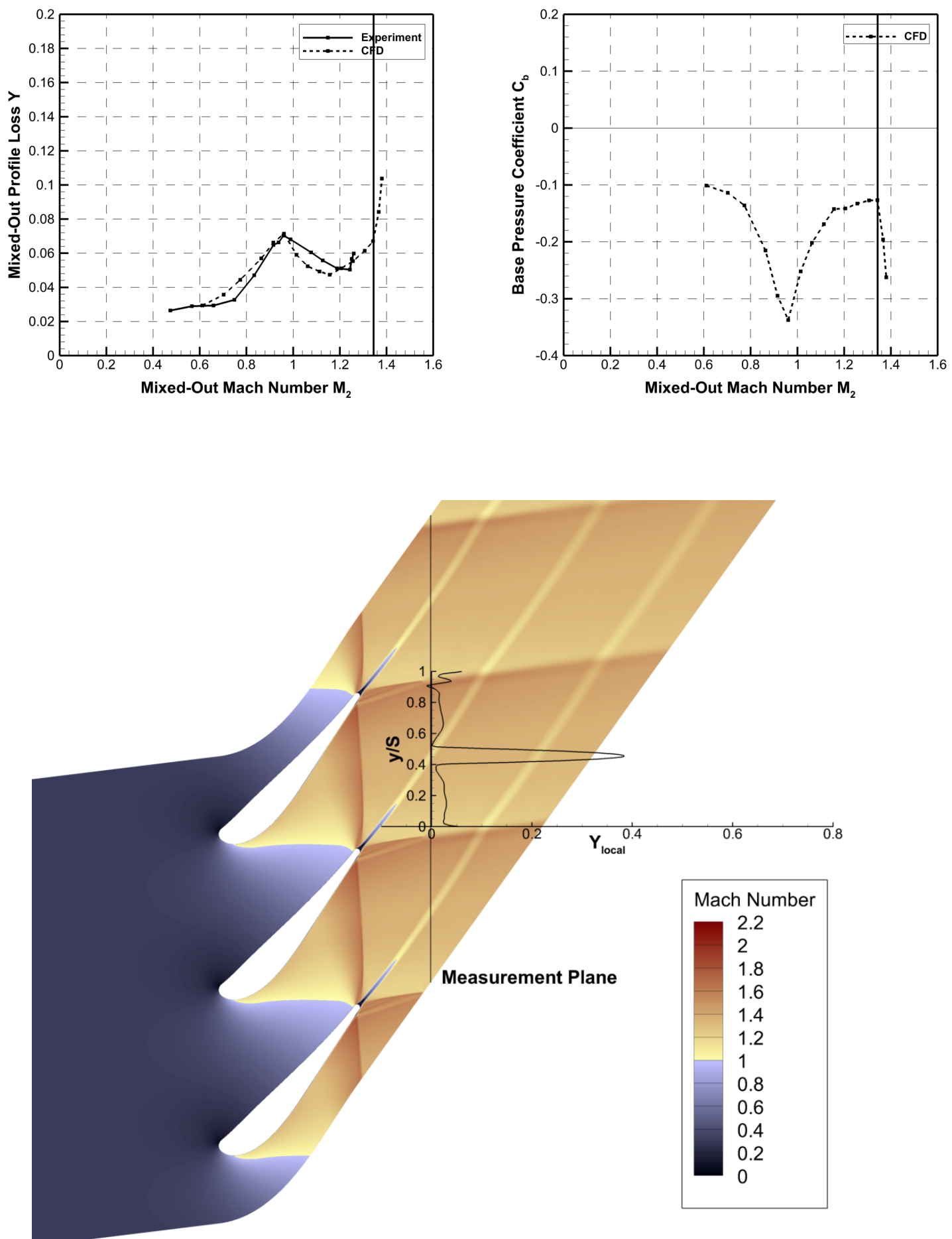

Figure 6.21: Blade-to-Blade Flow Features and Wake Profile of the AF4 Cascade at a Mixed-Out Mach Number of 1.34 (Profile Losses and Base Pressure Inset) 

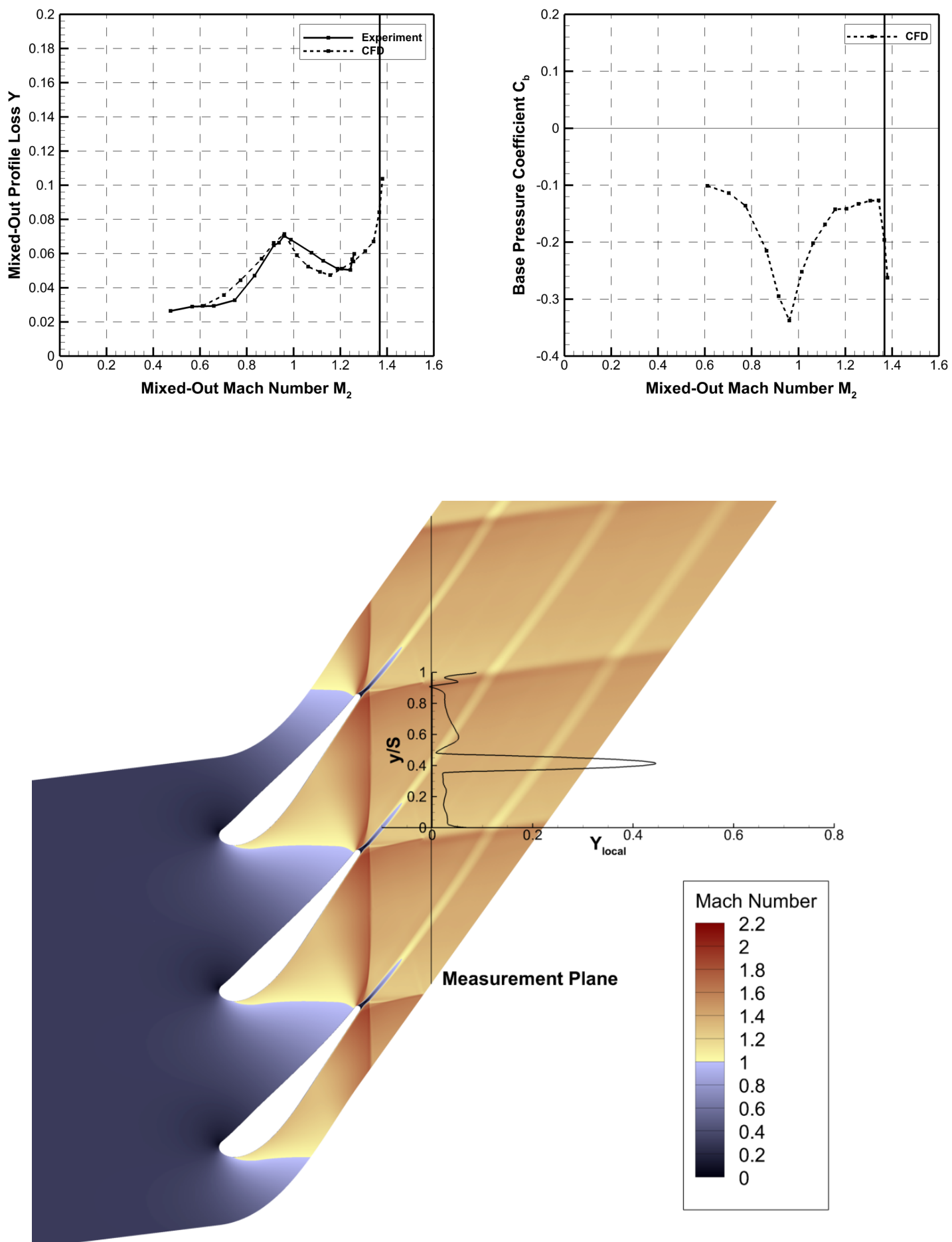

Figure 6.22: Blade-to-Blade Flow Features and Wake Profile of the AF4 Cascade at a Mixed-Out Mach Number of 1.37 (Profile Losses and Base Pressure Inset) 

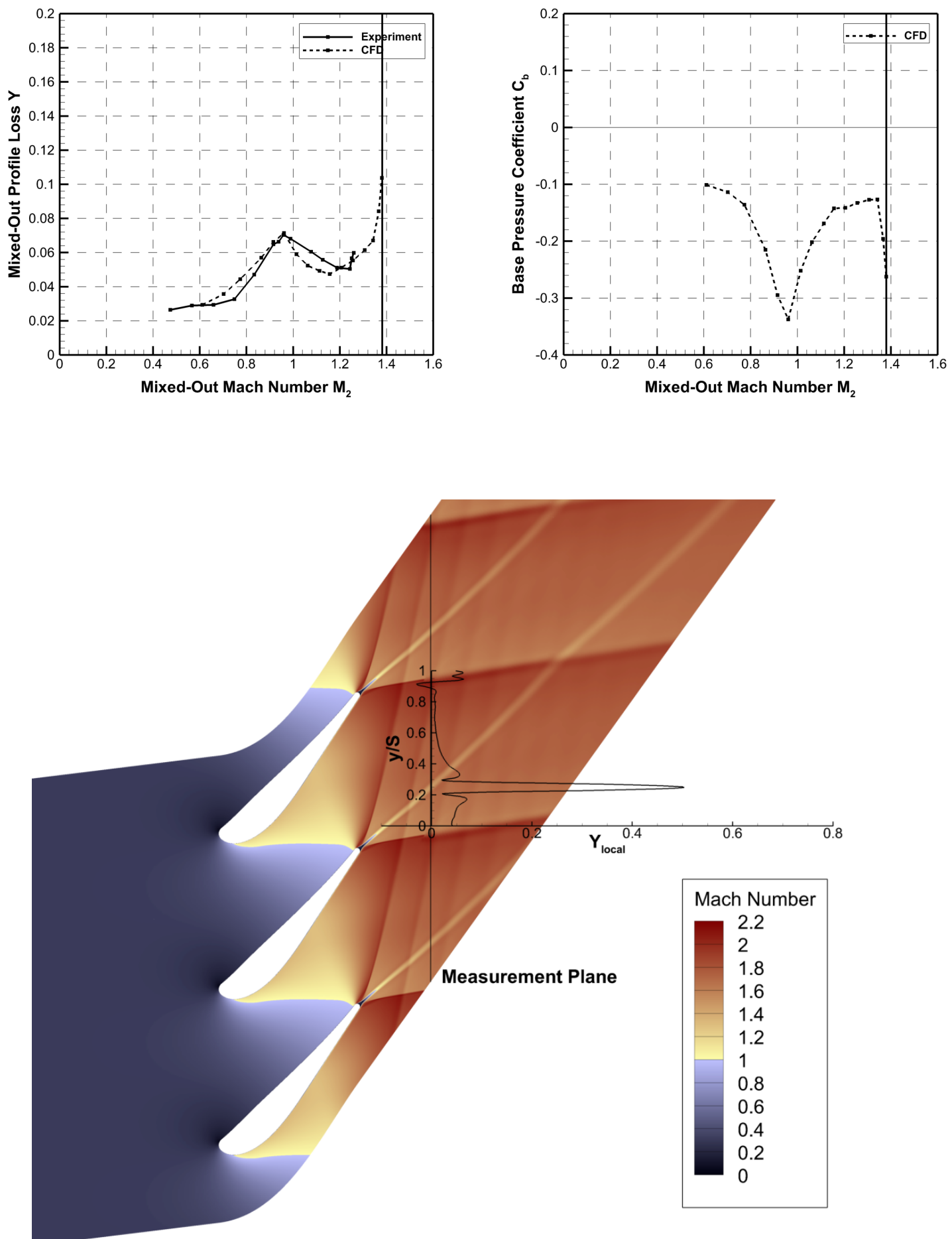

Figure 6.23: Blade-to-Blade Flow Features and Wake Profile of the AF4 Cascade at a Mixed-Out Mach Number of 1.38 (Profile Losses and Base Pressure Inset) 

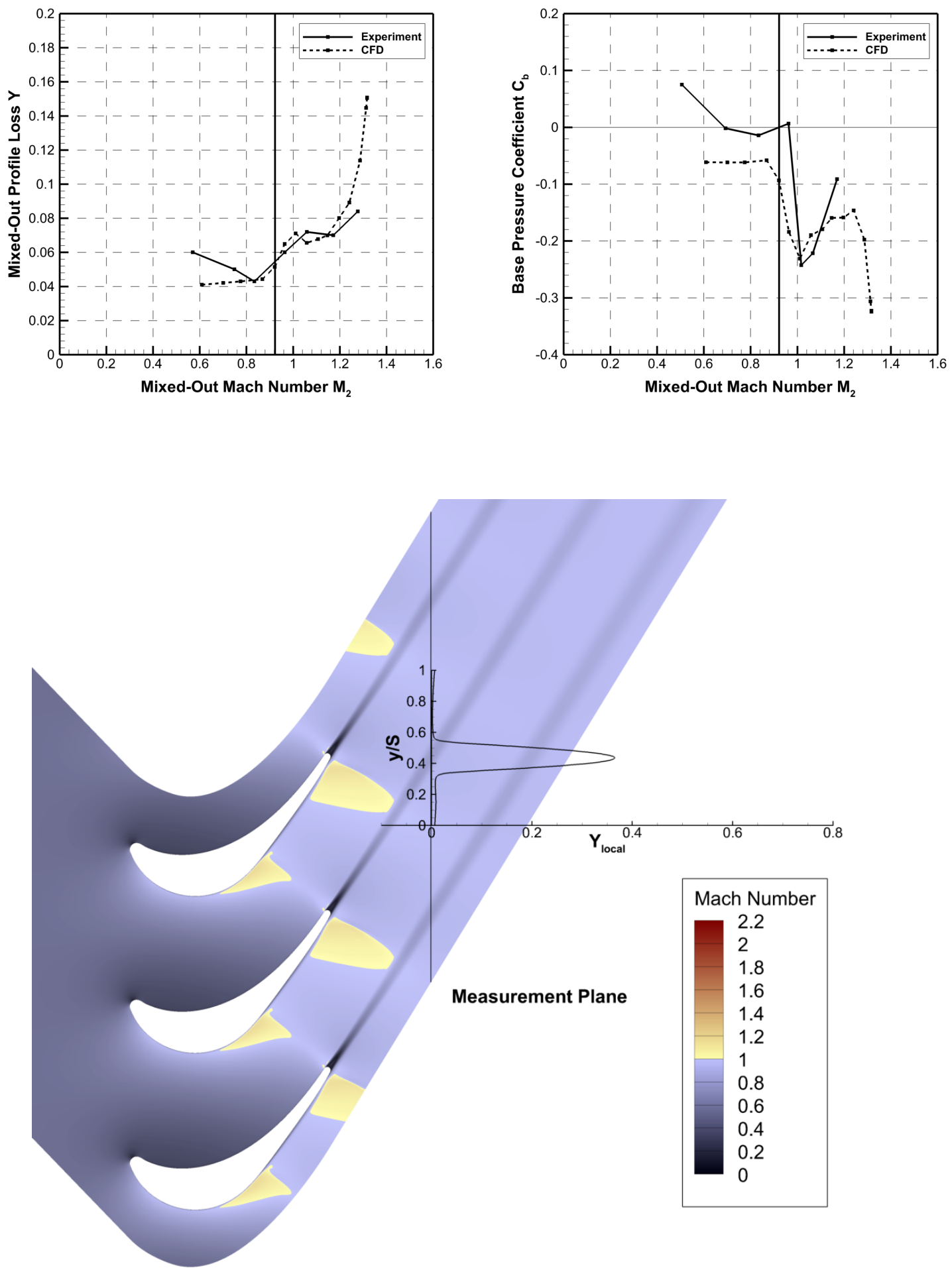

Figure 6.24: Blade-to-Blade Flow Features and Wake Profile of the HS1A Cascade at a Mixed-Out Mach Number of 0.92 (Profile Losses and Base Pressure Inset) 

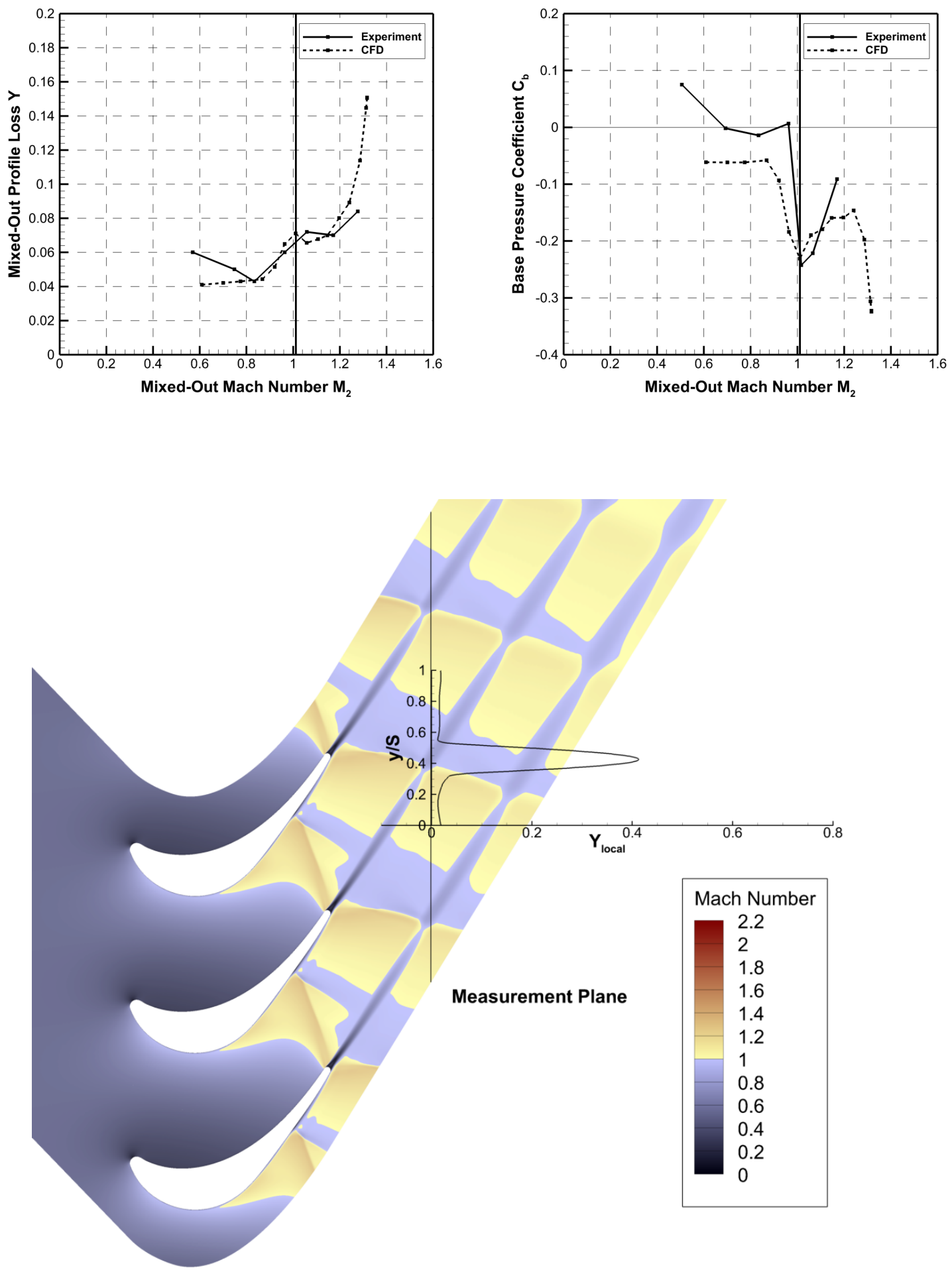

Figure 6.25: Blade-to-Blade Flow Features and Wake Profile of the HS1A Cascade at a Mixed-Out Mach Number of 1.01 (Profile Losses and Base Pressure Inset) 

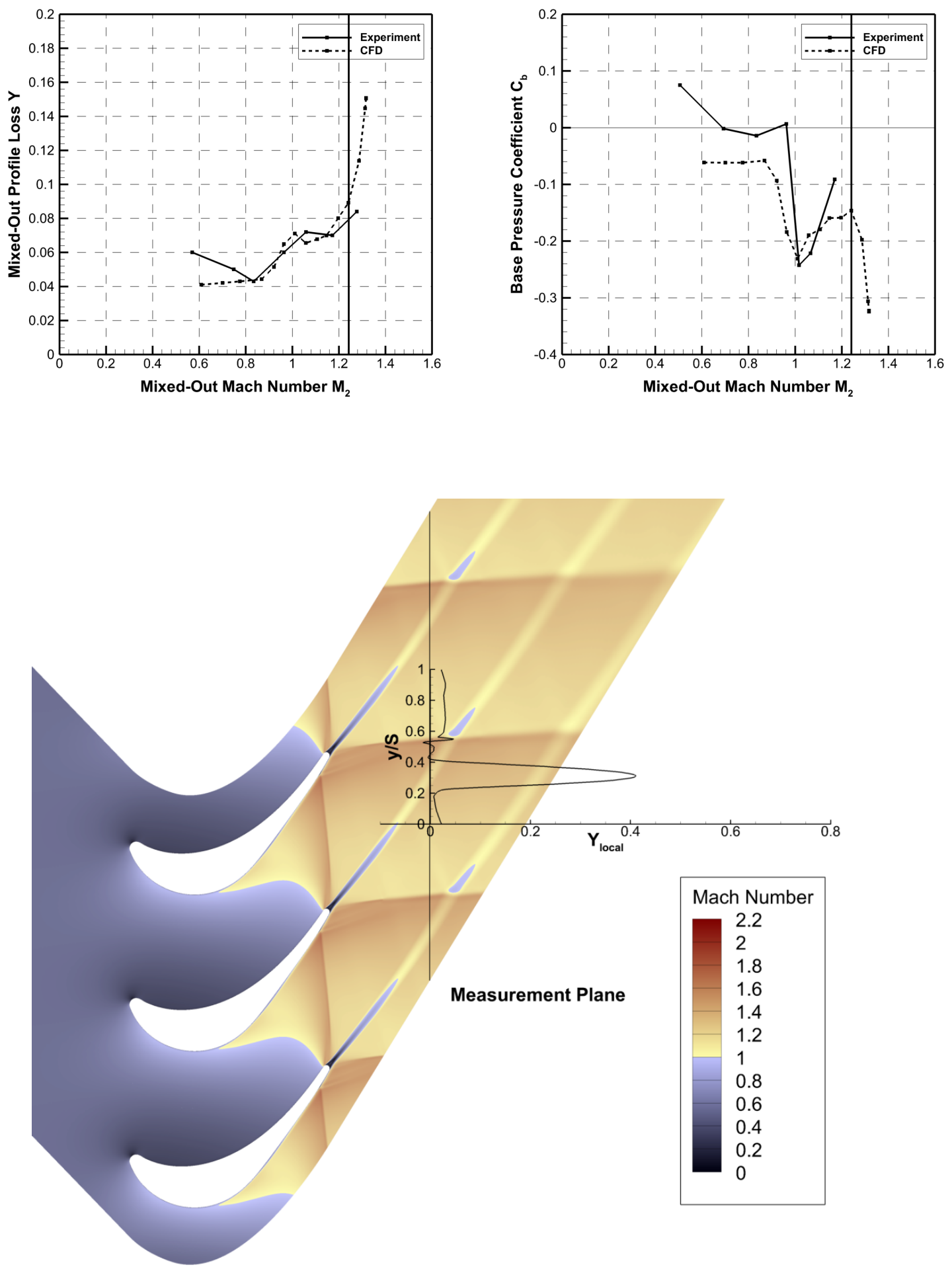

Figure 6.26: Blade-to-Blade Flow Features and Wake Profile of the HS1A Cascade at a Mixed-Out Mach Number of 1.24 (Profile Losses and Base Pressure Inset) 

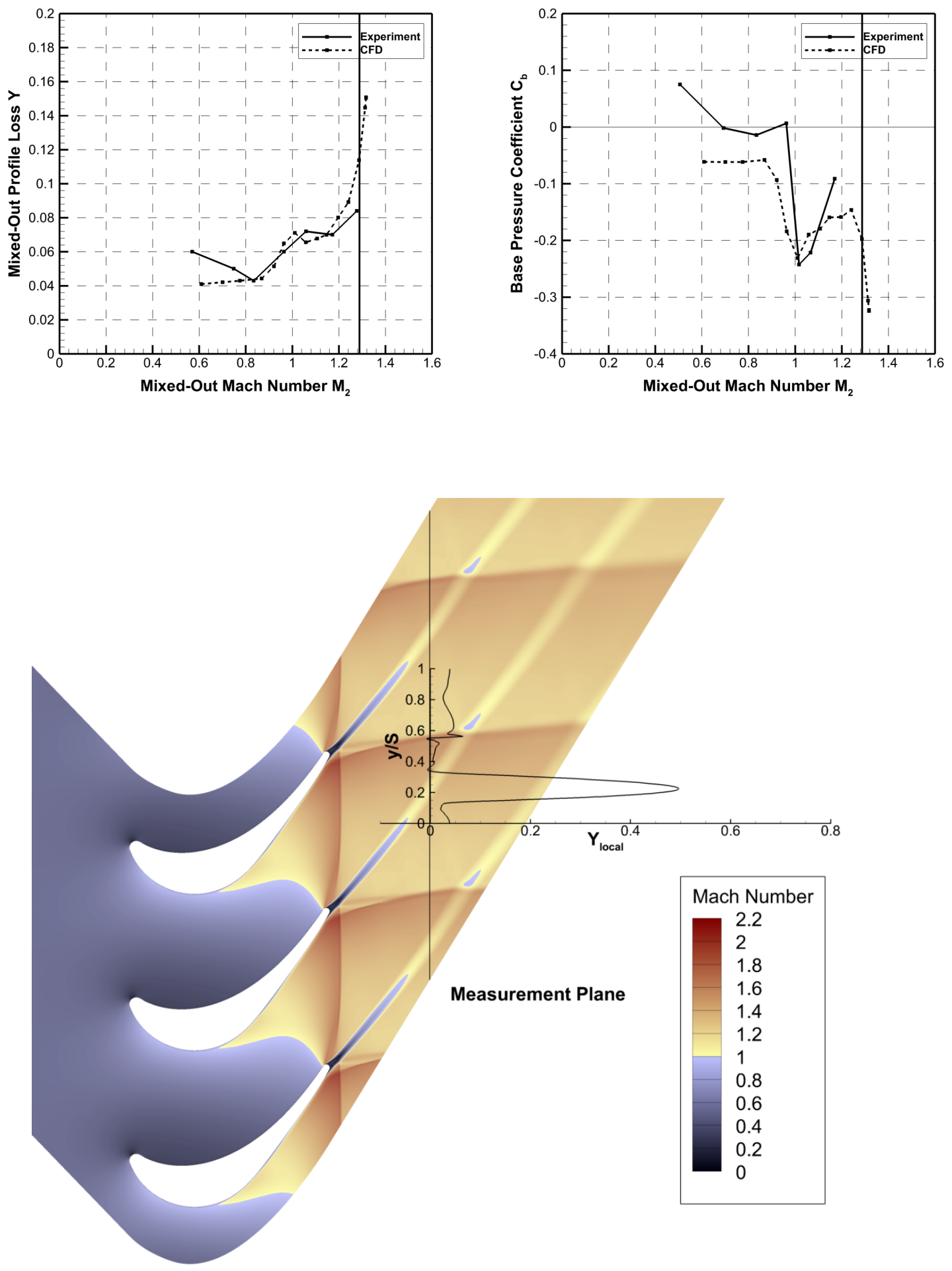

Figure 6.27: Blade-to-Blade Flow Features and Wake Profile of the HS1A Cascade at a Mixed-Out Mach Number of 1.29 (Profile Losses and Base Pressure Inset) 

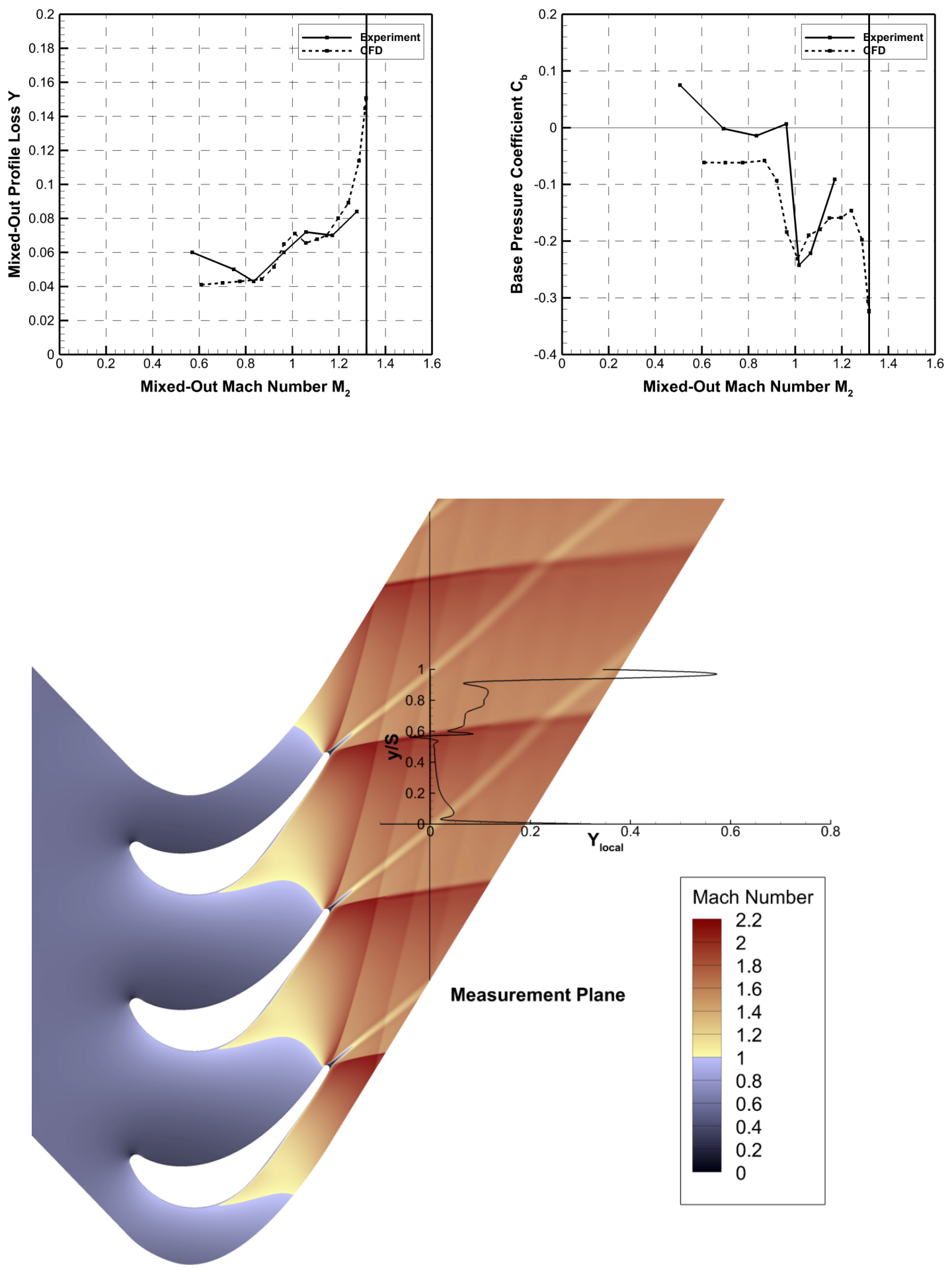

Figure 6.28: Blade-to-Blade Flow Features and Wake Profile of the HS1A Cascade at a Mixed-Out Mach Number of 1.32 (Profile Losses and Base Pressure Inset) 

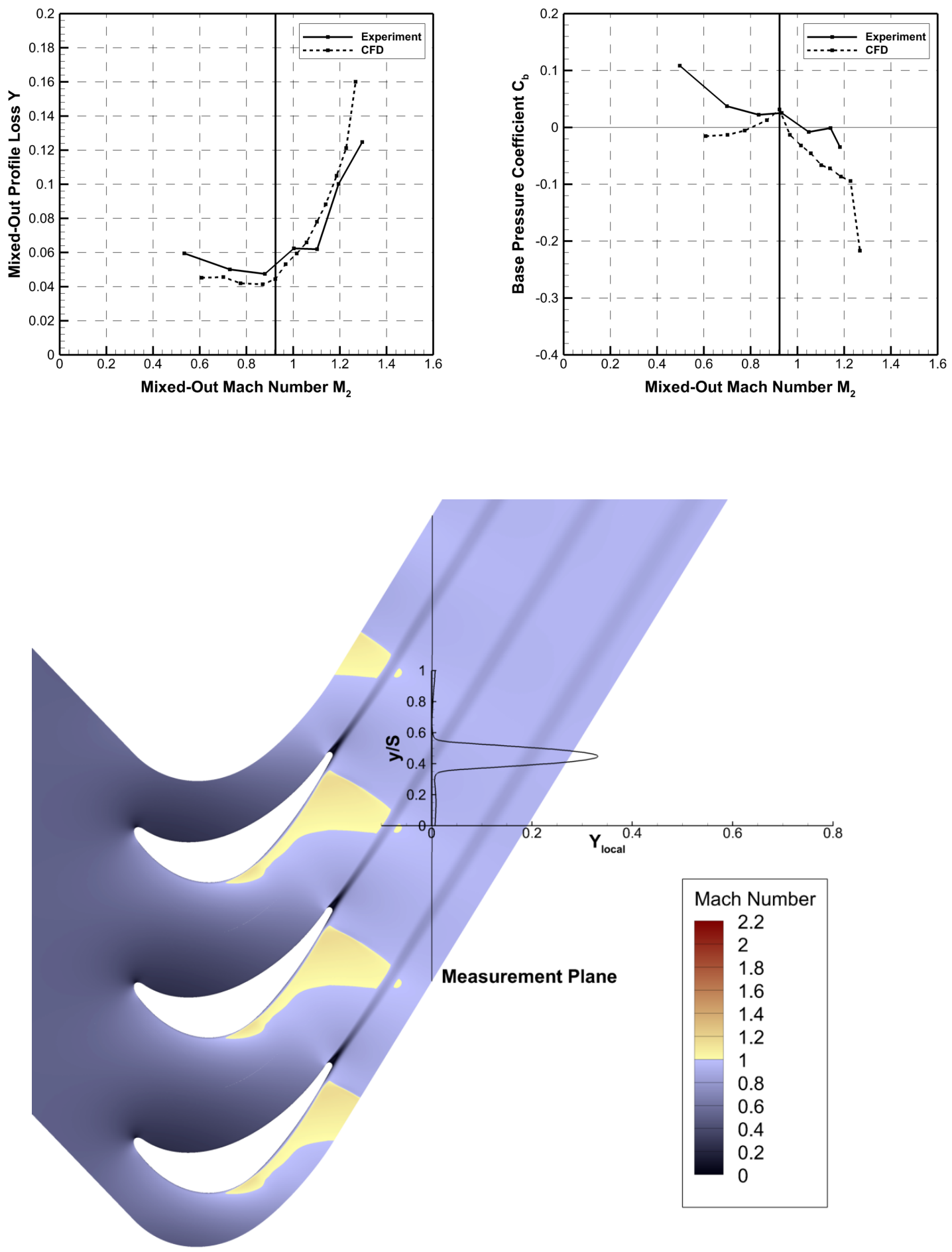

Figure 6.29: Blade-to-Blade Flow Features and Wake Profile of the HS1C Cascade at a Mixed-Out Mach Number of 0.92 (Profile Losses and Base Pressure Inset) 

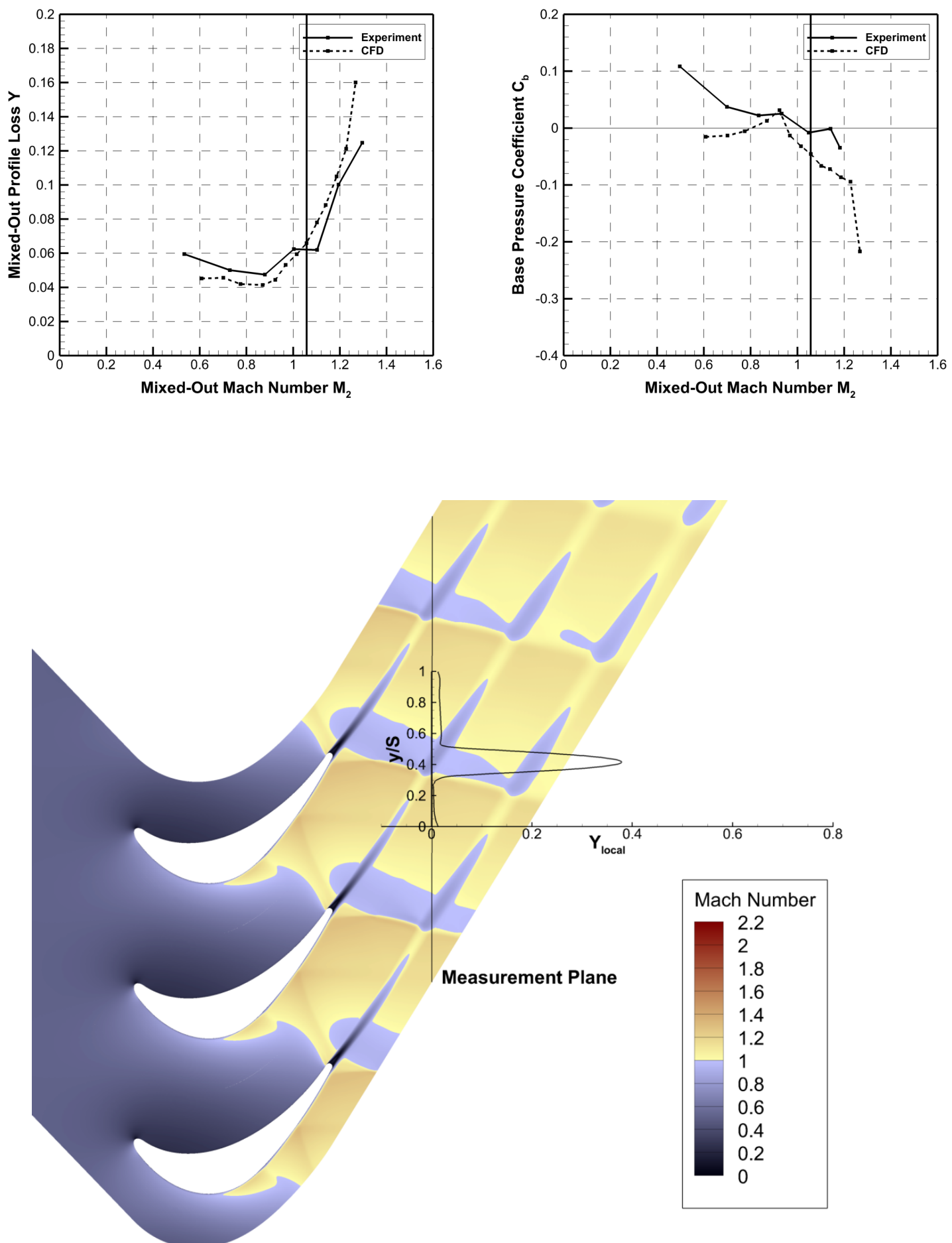

Figure 6.30: Blade-to-Blade Flow Features and Wake Profile of the HS1C Cascade at a Mixed-Out Mach Number of 1.06 (Profile Losses and Base Pressure Inset) 

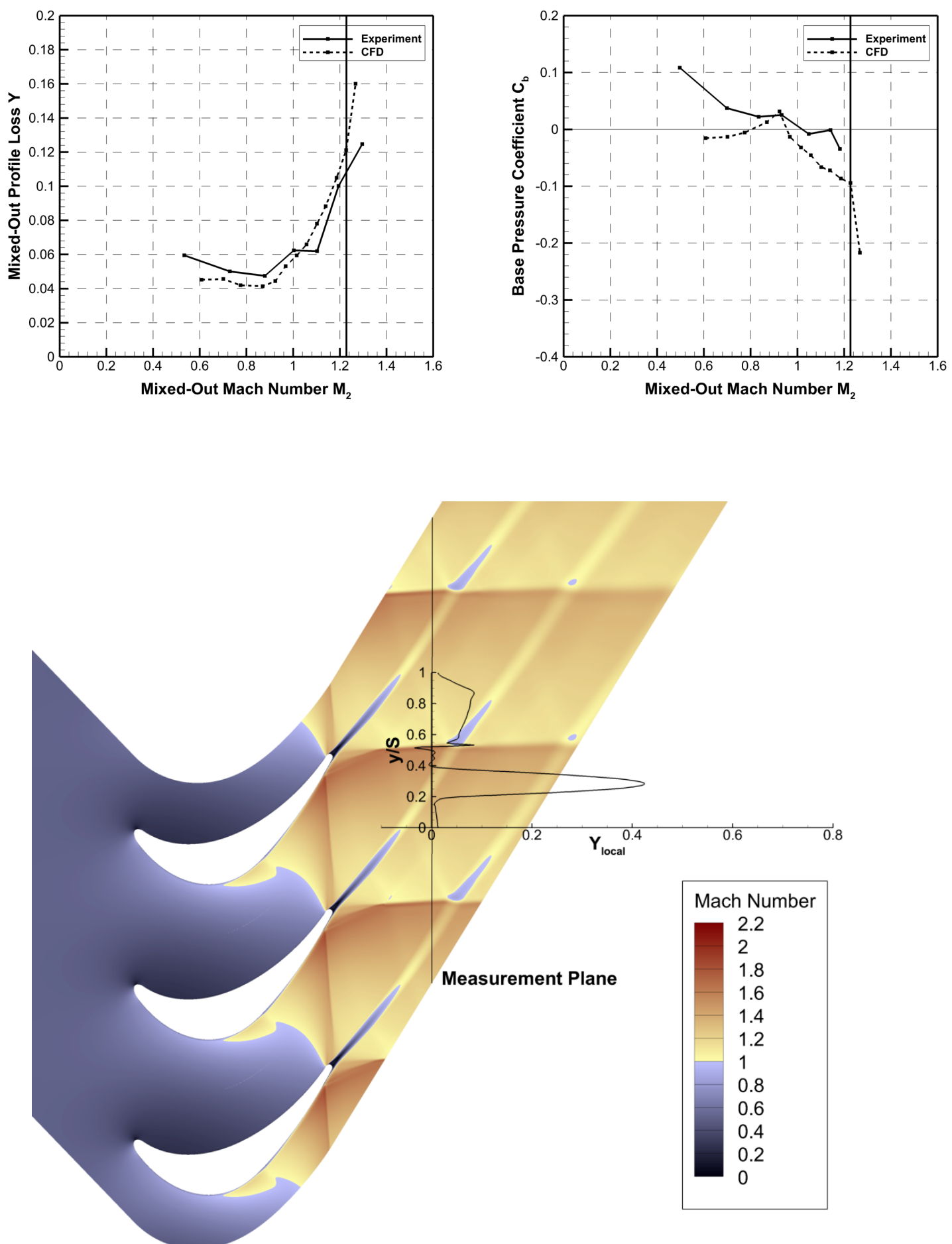

Figure 6.31: Blade-to-Blade Flow Features and Wake Profile of the HS1C Cascade at a Mixed-Out Mach Number of 1.23 (Profile Losses and Base Pressure Inset) 

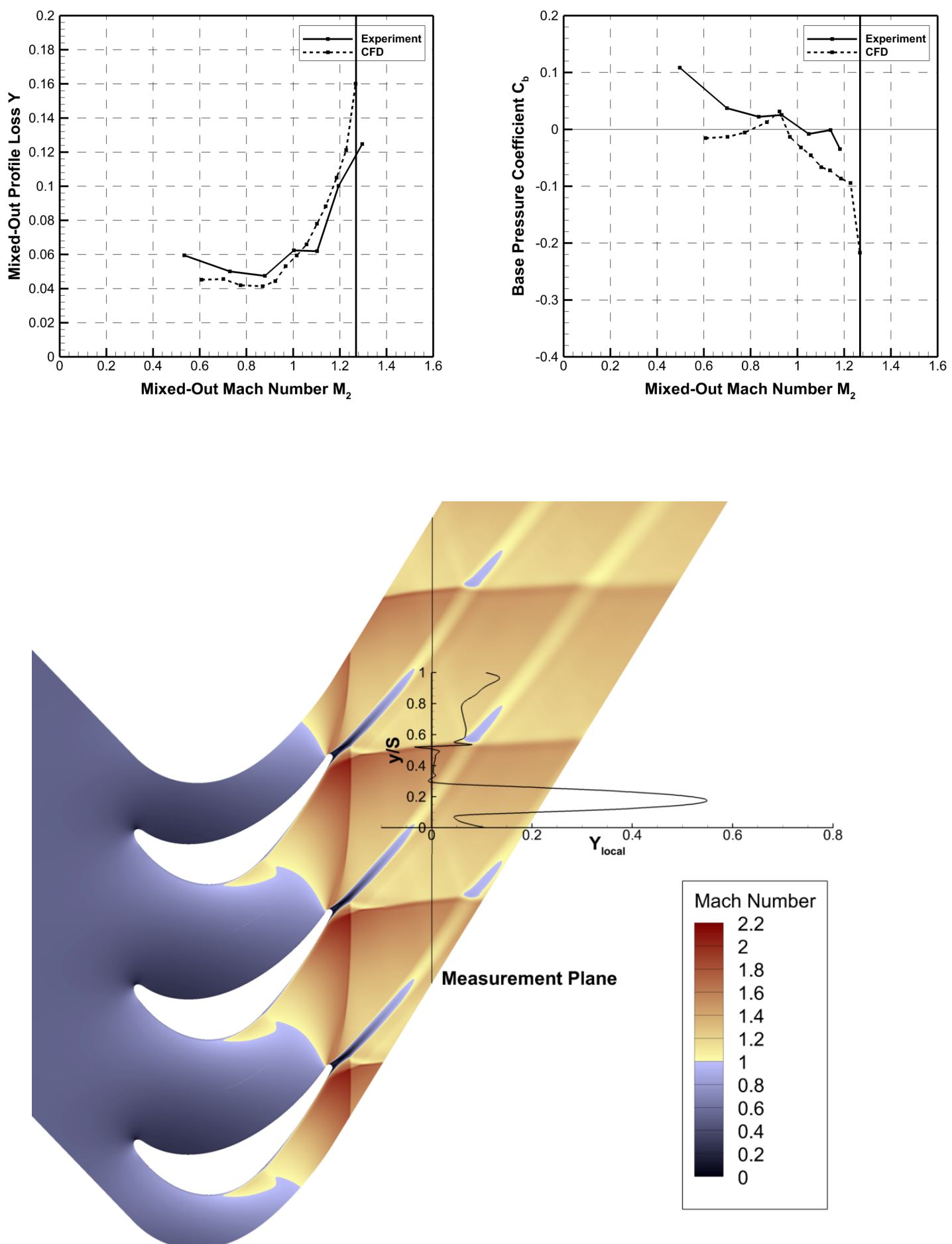

Figure 6.32: Blade-to-Blade Flow Features and Wake Profile of the HS1C Cascade at a Mixed-Out Mach Number of 1.27 (Profile Losses and Base Pressure Inset) 

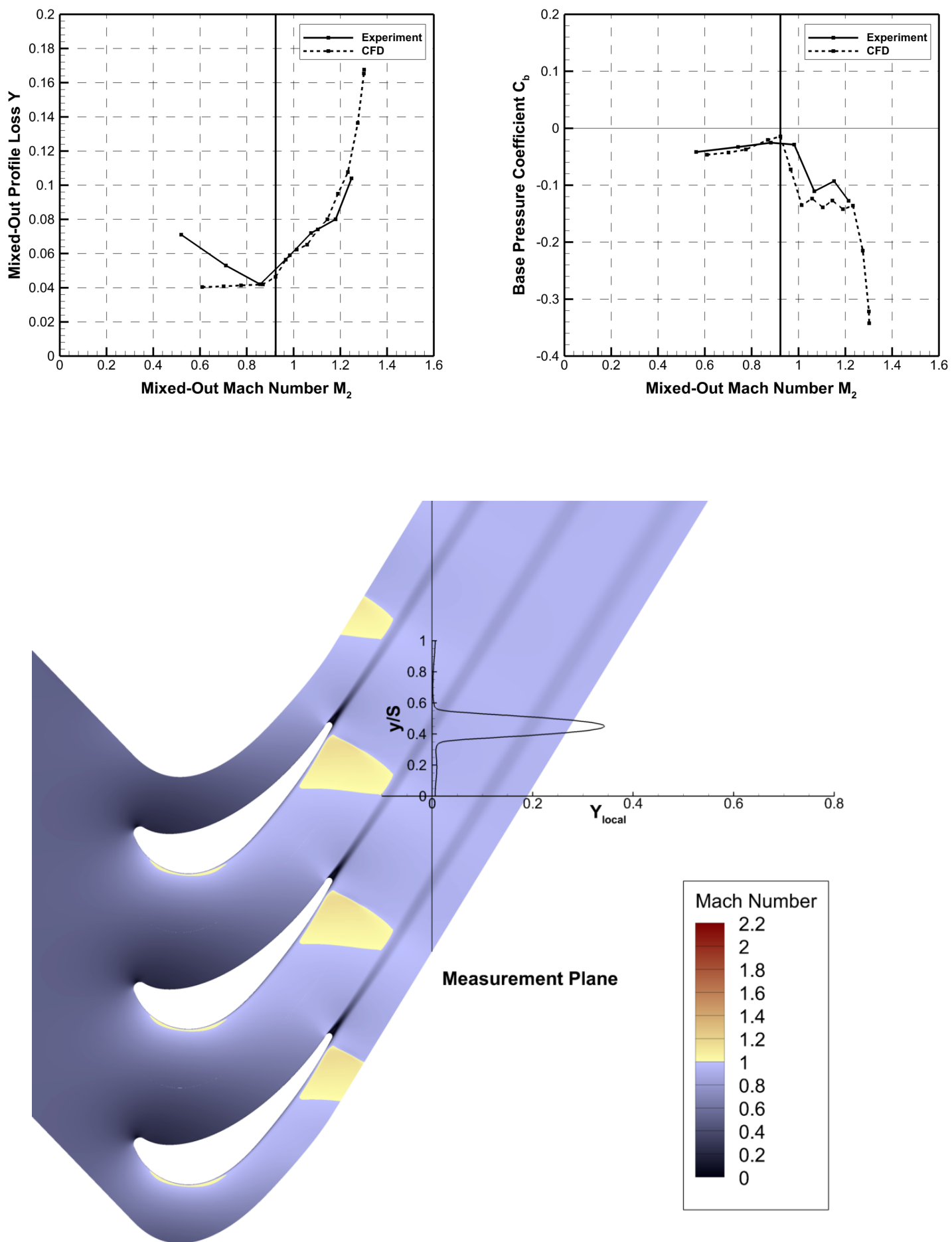

Figure 6.33: Blade-to-Blade Flow Features and Wake Profile of the HS1D Cascade at a Mixed-Out Mach Number of 0.92 (Profile Losses and Base Pressure Inset) 

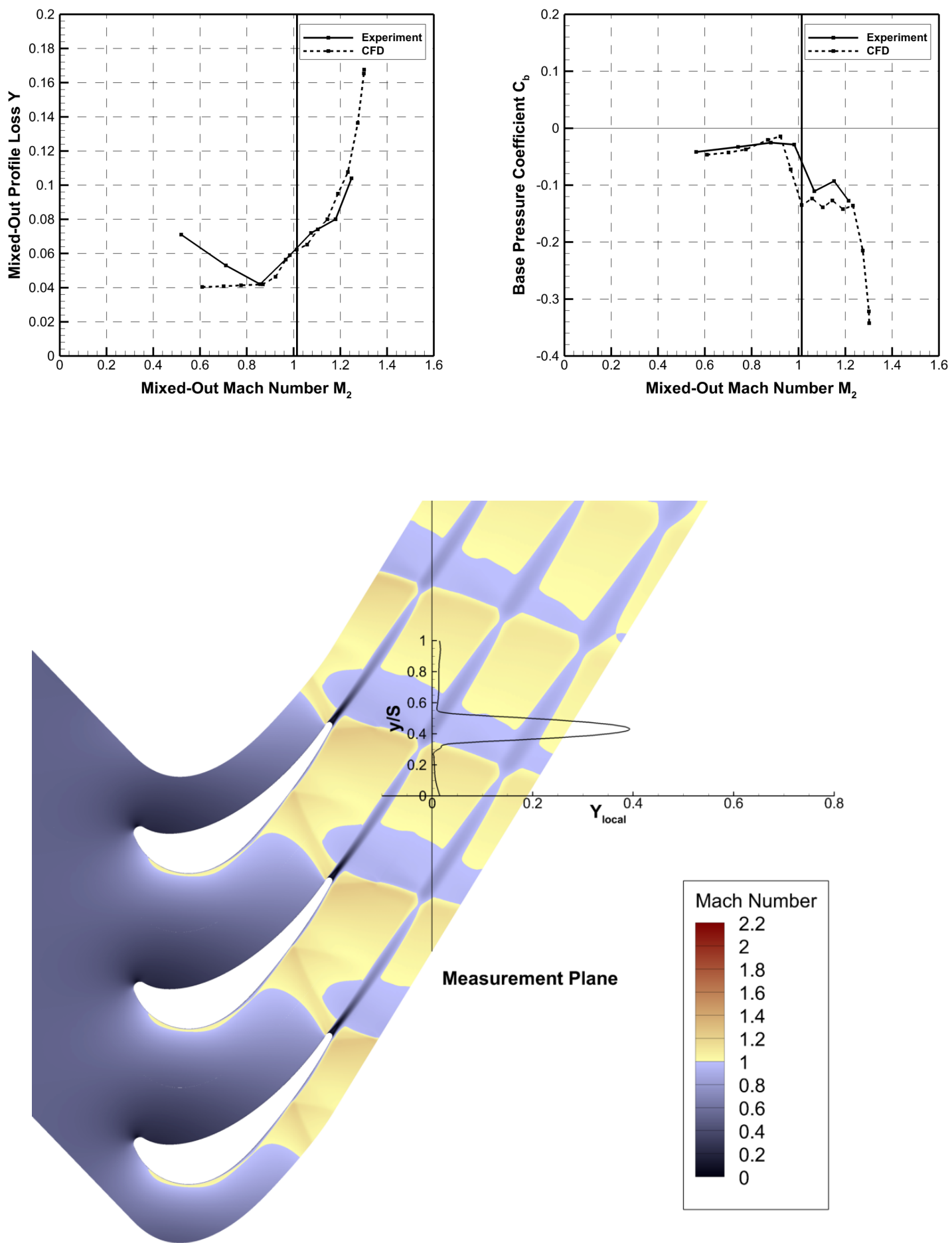

Figure 6.34: Blade-to-Blade Flow Features and Wake Profile of the HS1D Cascade at a Mixed-Out Mach Number of 1.01 (Profile Losses and Base Pressure Inset) 

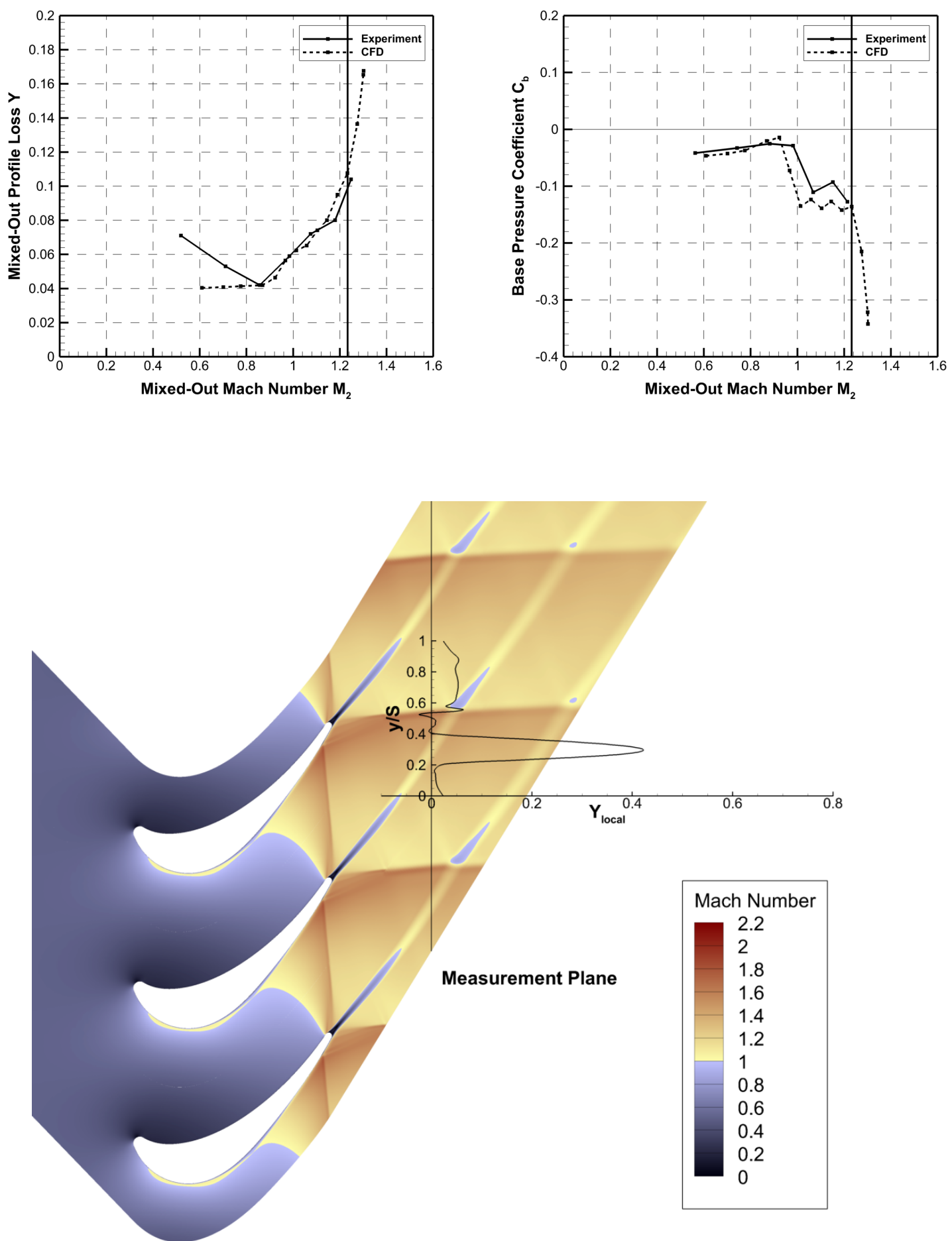

Figure 6.35: Blade-to-Blade Flow Features and Wake Profile of the HS1D Cascade at a Mixed-Out Mach Number of 1.23 (Profile Losses and Base Pressure Inset) 

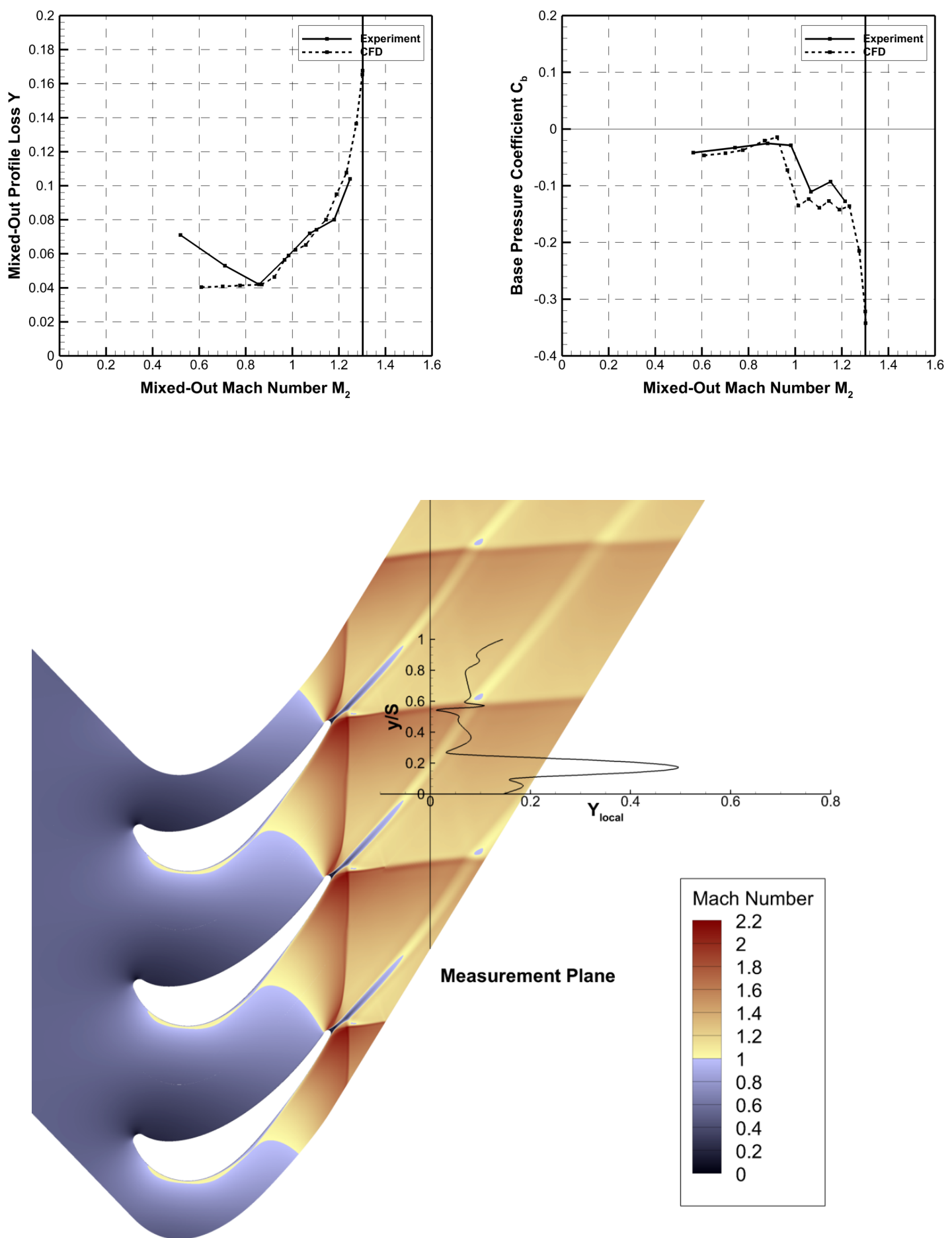

Figure 6.36: Blade-to-Blade Flow Features and Wake Profile of the HS1D Cascade at a Mixed-Out Mach Number of 1.30 (Profile Losses and Base Pressure Inset) 
edge, the base pressure likely causes the separated flows to turn towards each other to close up the separated region. A low base pressure would cause the flows to turn through a larger angle, thus requiring a stronger trailing edge shock to align the flows when they meet.

The AF4 cascade reaches a local minimum of profile loss coefficient in Figure 6.20, at an outlet Mach number of 1.16. The pressure side trailing edge shock is oblique and impinges on the suction surface of the adjacent blade. The shock-boundary layer interaction causes two closely-spaced parallel shocks to be "reflected" from the suction surface, which is expected based on the schematic of a shock-boundary layer interaction in Figure 2.7. Since this interaction is well away from the trailing edge, the position of this interaction does not seem to greatly affect the base pressure coefficient until higher Mach numbers are reached.

In Figure 6.21, at an outlet Mach number of 1.34, the trailing edge shocks approach the trailing edge of the adjacent blade, marking the beginning of a decrease in base pressure coefficient and an increase in profile losses. In Figure 6.22, at an outlet Mach number of 1.37, the trailing edge shocks have cleared the trailing edge of the adjacent blade, and have coalesced into a single shock just downstream of the cascade. From the wake profile in Figure 6.22, significant losses occur in the freestream outside of the trailing edge wakes as a result of this shock.

Increasing the downstream Mach number further results in the flow condition shown in Figure 6.23. The trailing edge shocks are highly oblique and have fully cleared the trailing edge of the adjacent blade. The outlet flow angle has significantly decreased and the trailing edge wakes have turned towards the axial direction.

The wake profile shown in Figure 6.23 has a small region of negative local loss coefficient $\left(Y_{\text {local }}\right)$ where a trailing edge shock crosses the measurement plane. Negative losses imply that the total pressure in the flow has increased, which cannot happen without work being done on the fluid. The exact cause of the negative values is uncertain, but might be attributed to computational errors such as approximations in the turbulence model. The two narrow regions of loss next to the trailing edge wake are also typical of CFD results, and are not considered physical. 
The Mach number contour plots for the HS1A cascade shown in Figures 6.24 to 6.28 show similar patterns to those observed in the AF4 cascade. The initial increase in losses near sonic outlet Mach numbers appears to result from the appearance of the trailing edge shock system, as shown in Figure 6.25. The base pressure decreases, causing increased losses in the wake and stronger trailing edge shocks. The increase in shock losses is confirmed by the increase in local loss in the freestream outside of the wake. This condition corresponds to the beginning of the loss plateau.

The loss plateau of the HS1A cascade appears to be closely related to the base pressure, which increases only slightly until an outlet Mach number of 1.24, as shown in Figure 6.26. Above this condition, the profile losses increase and the base pressure decreases as a result of the trailing edge shock approaching and crossing the trailing edge of the adjacent blade, as shown in Figures 6.26 and 6.27. In Figure 6.28, at an outlet Mach number of 1.32, the trailing edge shocks are sufficiently oblique to clear the trailing edge of the adjacent blade. High shock losses are observable in the wake profile, outside of the trailing edge wakes.

Figure 6.29 shows the HS1C cascade at an outlet Mach number of 0.92, at which point the profile losses are minimized. The HS1C cascade has a high value of base pressure at this condition. As the outlet Mach number is increased, the base pressure steadily decreases. The trailing edge shocks are gradually strengthened.

The behaviour shown in the contour plots of the HS1D cascade resembles that of the HS1A cascade. However, unlike the HS1A cascade, the long, weak adverse pressure gradient that covers a large proportion of the HS1D suction surface causes thickening of the boundary layer, which is visible as a dark narrow region of subsonic flow along the suction surface. At higher Mach numbers, such as in Figure 6.35 showing an outlet Mach number of 1.23, this boundary layer thins near the aft of the blade as a result of the expansion fan generated by the trailing edge of the adjacent blade.

The boundary layers near the trailing edge of the cascades will be characterized more quantitatively in the next section. 


\subsection{Blade Surface Boundary Layers}

From the base pressure results in Section 6.7, the Sieverding et al. (1979) base pressure correlation does not fully capture the base pressure behaviour based on the trailing edge wedge angle $\left(W_{e}\right)$ and unguided turning angle $\left(\theta_{u}\right)$. The HS1A and HS1D cascades have the same values of these parameters but do not show the same base pressure behaviour. Given that low base pressures are associated with high flow entrainment in the separated shear layer next to the base region, it is relevant to investigate the boundary layers of the blade surfaces near the trailing edge in order to better understand the base pressure behaviour.

The suction surface and pressure surface boundary layers were extracted from the CFD results just upstream of the trailing edge (at $0.95 C_{x}$ ). The boundary layer edge was defined as $99.5 \% V_{e}$ in this investigation, where $V_{e}$ is the edge velocity. The selection of a satisfactory boundary layer edge criterion was challenging. For a flat plate boundary layer, the flow outside of the boundary layer has a uniform velocity, often called the edge velocity $\left(V_{e}\right)$. In a cascade blade row, the blade-to-blade static pressure gradient implies that there is also a blade-toblade velocity gradient, with the highest flow velocities occurring near the suction surface. It thus becomes difficult to define a single value for the edge velocity. It was considered that a more appropriate boundary layer edge criterion might be based on the total pressure in the flow. Given that the total pressure decreases in the boundary layer, the boundary layer edge could be defined as the point where the total pressure reaches the value of the total pressure upstream of the cascade. In other words, the edge of the boundary layer is where inviscid flow is reached. However, at transonic and supersonic Mach numbers, the shocks that occur in the flow cause a reduction in total pressure even outside of the boundary layers, making it difficult to define the boundary layer edge in this way. For the results of the current investigation, a different edge velocity was defined for the suction and pressure surfaces. The velocity profile in the suction surface boundary layer reaches a maximum value, then begins to decrease as a result of the blade-to-blade velocity gradient. The edge velocity was defined as this maximum value. For the pressure surface boundary layer, the edge velocity was obtained at the point where the velocity distribution becomes approximately linear, as a result of the blade-to-blade 
velocity gradient. The boundary layer edge for both the suction and pressure surfaces was then defined as the point where the velocity reaches $99.5 \%$ of this "edge velocity" $V_{e}$.

Three parameters were calculated from the boundary layer velocity profile data: the displacement thickness $\left(\delta^{*}\right)$, the momentum thickness $(\theta)$ and the shape factor $(H)$. The displacement thickness is defined for compressible flow as:

$$
\delta^{*}=\int_{0}^{y_{e}}\left(1-\frac{\rho(y) V(y)}{\rho_{e} V_{e}}\right) \mathrm{d} y
$$

where $y$ is the distance normal to the surface. The subscript $e$ indicates a value at the edge of the boundary layer, as defined above. The momentum thickness is defined for compressible flow as:

$$
\theta=\int_{0}^{y_{e}} \frac{\rho(y) V(y)}{\rho_{e} V_{e}}\left(1-\frac{V(y)}{V_{e}}\right) \mathrm{d} y
$$

The shape factor is defined using the displacement thickness and the momentum thickness, as follows:

$$
H=\frac{\delta^{*}}{\theta}
$$

The shape factor is a measure of the fullness of a boundary layer velocity profile. Lower values of shape factor correspond to a fuller velocity profile, with greater fluid momentum near the wall. Higher values of shape factor indicate that the boundary layer may be approaching separation.

The displacement thicknesses and momentum thicknesses of the four cascades are plotted against the outlet mixed-out Mach number in Figure 6.37. The dashed portions of the line correspond to the cases in which the trailing edge shock from the adjacent blade crosses the boundary layer traverse, making it difficult to assign an edge velocity.

The pressure surface boundary layers are about $20 \%$ to $50 \%$ thinner than the suction surface boundary layers. Additionally, at transonic and supersonic Mach numbers, the flow through the blade passage is generally choked, with subsonic flow over the pressure surface. As previously seen in the blade loadings in Figure 6.7, this pressure side flow does not change significantly with the outlet Mach number over the range of Mach numbers examined. The 


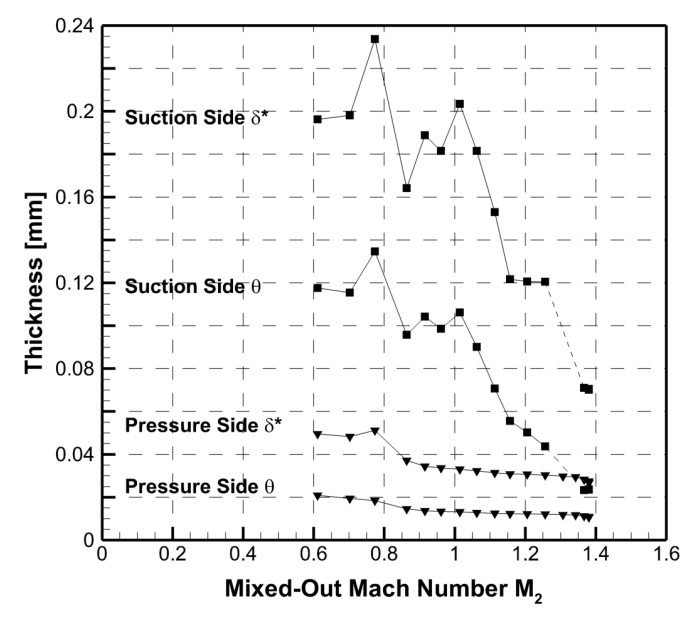

(a) AF4 Cascade

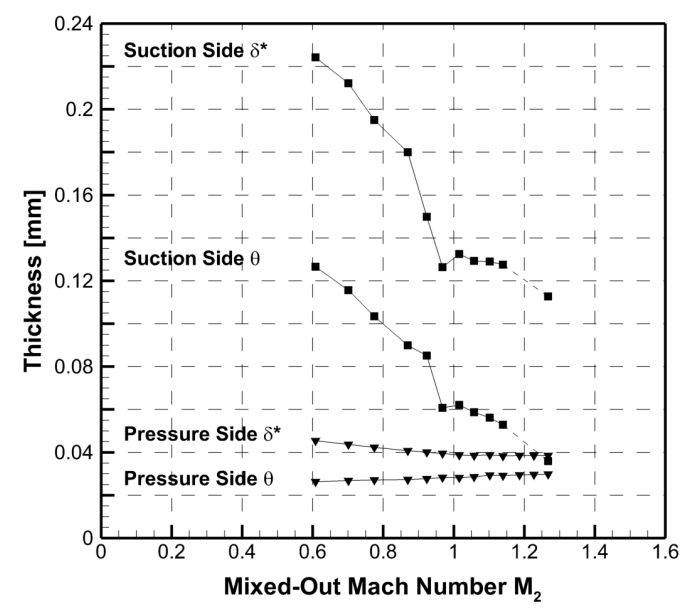

(c) HS1C Cascade

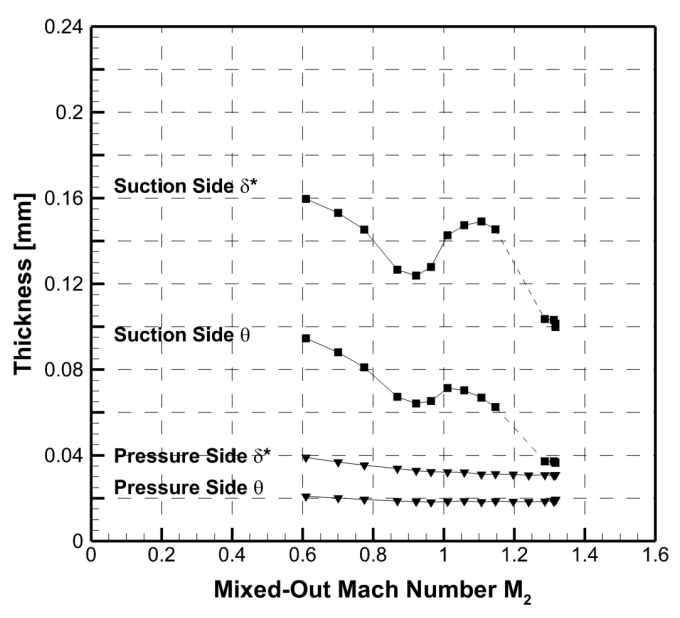

(b) HS1A Cascade

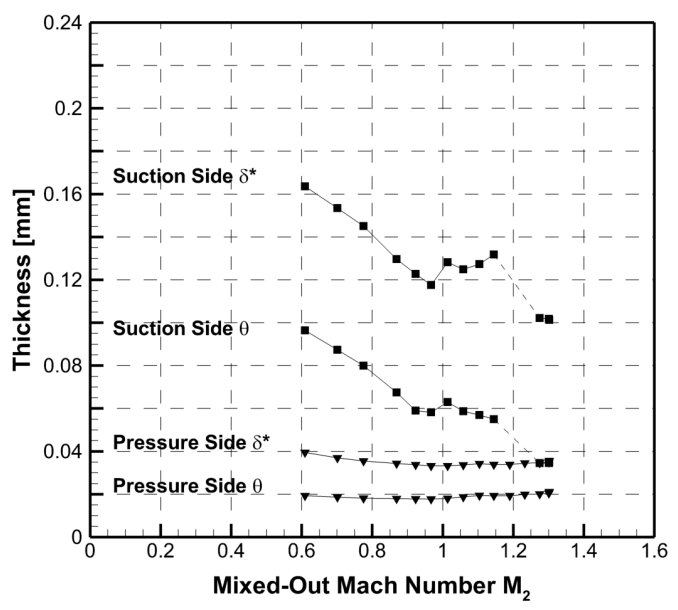

(d) HS1D Cascade

Figure 6.37: Effect of Mach Number on the Boundary Layer Displacement Thickness $\left(\delta^{*}\right)$ and Momentum Thickness $(\theta)$ at $95 \%$ Axial Chord 
choked flow and lower velocities over the pressure surface contribute to the approximately constant behaviour of the pressure side boundary layer parameters in Figure 6.37. Since the pressure side boundary layers are thinner and do not greatly evolve with increasing Mach number, they are not expected to have a strong effect on the profile losses or the base pressure trends observed in previous sections.

Unlike the flows near the pressure surfaces, the flows near the suction surfaces have higher velocities, often supersonic, and are a strong function of the outlet Mach number. For the four cascades, the displacement and momentum thicknesses undergo an overall decrease with increasing outlet Mach number. The flow becomes increasingly accelerated through the blade passage, causing the boundary layers to thin. In particular, at outlet Mach numbers just below 1.0, the HS1 cascades all exhibit a decrease in displacement and momentum thickness. Around this outlet Mach number, regions of supersonic flow begin to appear near the trailing edge, as seen previously in the Mach number contour plots in Figures 6.24, 6.29 and 6.33. The flow acceleration associated with the occurrence of supersonic flow likely causes the boundary layer to thin. As the flow becomes transonic and the outlet Mach number is increased, the shock-boundary layer interaction appears on the suction surface and gains strength, resulting in a strong local adverse pressure gradient which has a thickening effect on the boundary layer. At the highest outlet Mach numbers simulated, the highly oblique shocks from the adjacent blade sweep past the trailing edge, and the boundary layer is no longer thickened by the shock-boundary layer interaction.

The effect of the outlet Mach number on the shape factor of the suction surface boundary layer is shown in Figure 6.38 for the four cascades. Over the range of outlet Mach numbers examined, the shape factor increases for the four cascades. The shape factor trends for the four cascades are very similar, with the trend lines slightly offset in the vertical direction. Interestingly, the order in shape factor magnitude of the four cascades is also the order in base pressure values in Figures 6.14 to 6.17. For example, the cascade with the highest shape factor trend line is HS1C, which is the cascade with the highest value of Sieverding et al. (1979) parameter $\left(\Phi=\frac{W_{e}+\theta_{u}}{2}=10.25^{\circ}\right)$. From Figure 6.15. HS1C also showed the highest values of base pressure when compared to the other cascades. This connection can be explained as 


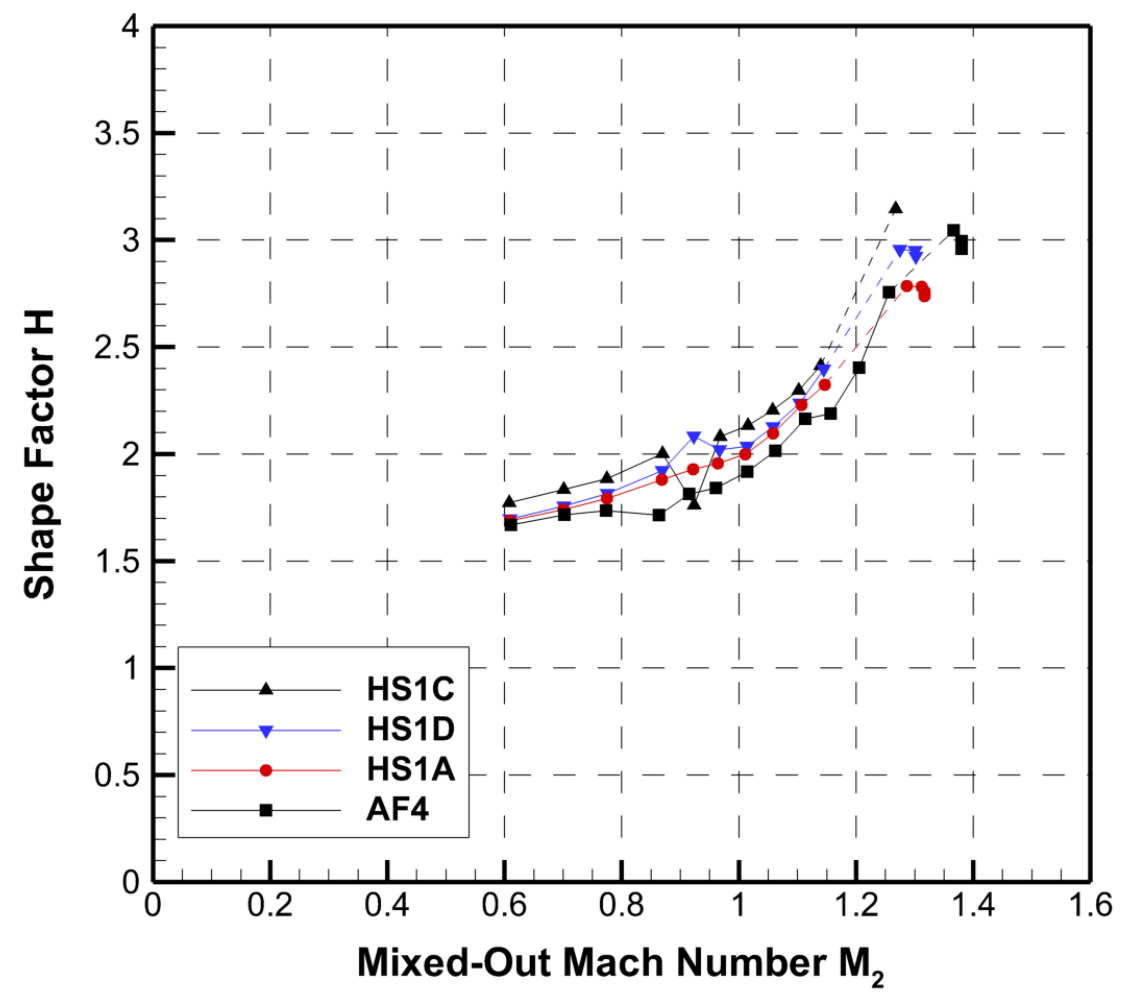

Figure 6.38: Effect of the Outlet Mach Number on the Shape Factor of the Suction Surface Boundary Layer at 95\% Axial Chord 
follows. The higher boundary layer shape factor implies that the boundary layer has a less full velocity profile, with less fluid momentum in the near-wall region. As a result, when the boundary layer separates at the trailing edge, the entrainment power of the shear layer is reduced and the base pressure remains high.

The HS1C exhibits an anomalous dip in shape factor at a mixed-out Mach number of 0.92. Referring back to the Mach number contour plot shown in Figure 6.29, a region of supersonic flow near the suction surface ends in a normal shock. The shock-boundary layer interaction occurs just downstream of the boundary layer traverse line $\left(0.95 C_{x}\right)$. The over-expansion that occurs just upstream of the shock is likely responsible for the anomalous low shape factor. This data point is not considered representative of the boundary layer at the trailing edge.

The shape factor is an indicator of the character of the streamwise pressure gradient (White, 2006). The higher shape factors observed in the HS1C and HS1D cascades, for example, suggest the presence of adverse pressure gradients in the boundary layer upstream of the point of interest. Recalling the blade loadings shown in Figure 6.7, the aft-loaded HS1C has a stong adverse pressure gradient after the suction peak. The front-loaded HS1D cascade has a weak adverse pressure gradient covering a large portion of the blade chord. These adverse pressure gradients reduce the momentum in the boundary layers, causing higher values of shape factor.

The shape factor trends help to explain the differences in base pressure trends between the HS1A and HS1D cascades. These two cascades have the same value of $\Phi$, but the HS1D cascade exhibits higher base pressure values. In Figure 6.38, the HS1D cascade has a slightly higher shape factor than the HS1A cascade, particularly just below an outlet Mach number of 1.0. The boundary layer near the trailing edge, having lower fluid momentum near the surface, has a reduced entrainment power over the base region, leading to a higher base pressure.

The AF4 cascade has lowest value of shape factor. As discussed previously, the AF4 cascade exhibits a strong adverse pressure gradient along its suction surface, causing relatively large values of the boundary layer integral length scales. It is thus surprising that the shape factor would be low at the trailing edge, after such strong flow deceleration. The AF4 cascade has the lowest value of the Sieverding et al. (1979) parameter, indicating low suction side 
curvature downstream of the throat. For this reason, it is hypothesized that the momentum in boundary layer is restored as the flow moves downstream along the nearly straight tail of the blade. The AF4 cascade also turns the flow through a smaller angle than HS1 cascades, so it may be more tolerant of an adverse pressure gradient. Perhaps an airfoil with similar flow turning but a less strong region of adverse pressure gradient would have an even lower shape factor. In any case, the AF4 cascade's behaviour once again supports the idea that a low shape factor in the boundary layer near the trailing edge leads to a low base pressure.

\subsection{Denton (1993) Trailing Edge Loss Prediction}

In previous sections, the behaviour of the base pressure and the boundary layer parameters near the trailing edge have been presented. These have been accompanied with qualitative descriptions of how their behaviour affects the total pressure losses. In order to have a better sense of the relative contributions of these variables to the losses, it is useful to return to the expression by Denton (1993) for trailing edge losses. The trailing edge losses are expressed as a total pressure loss coefficient:

$$
Y_{T E}=\frac{\Delta P_{o}}{0.5 \rho V_{r e f}^{2}}=-\frac{C_{b, D} t}{w}+\frac{2 \theta}{w}+\left(\frac{\delta^{*}+t}{w}\right)^{2}
$$

where $\theta$ and $\delta^{*}$ are the boundary layer momentum thickness and displacement thickness respectively, $t$ is the trailing edge thickness, $w$ is analogous to the throat opening $(w=t+o)$ and $C_{b, D}$ is the base pressure coefficient. The base pressure coefficient is defined by Denton (1993) as:

$$
C_{b, D}=\frac{P_{b}-P_{r e f}}{0.5 \rho V_{r e f}^{2}}
$$

where $P_{b}$ is the base pressure, $P_{r e f}$ is a reference static pressure and $V_{r e f}$ is the flow velocity at the same reference point. The choice of reference point will be explored further, below.

Some steps of the derivation were left out by Denton (1993). The full derivation is provided in Appendix B. The derivation contains a number of significant simplifying assumptions. Firstly, incompressible flow is assumed, while the flows encountered in the current investigation 
are clearly compressible. Secondly, it is assumed that the flow across the blade throat is uniform, while the flow in real blade rows has cross-passage distributions of pressure, velocity and density. One particularly problematic aspect of the derivation is that Denton selected the uniform flow conditions at the throat as the reference conditions for the total pressure loss coefficient $Y_{T E}$ and for the base pressure coefficient $C_{b, D}$. In reality, no such uniform flow conditions exist at the throat. The base pressure coefficient used throughout the current study is defined based on the outlet mixed-out conditions, and is thus defined differently than the form used in Denton's expression. Undoubtedly, Denton selected the throat plane as a reference condition because the expression resulting from the derivation is simple and easy to interpret. Therefore, Denton s expression may not have been meant to be applied directly to real blade rows, but was rather meant to identify and show the relative magnitudes of the loss contributions for a simplified case. With this scope in mind, the base pressure and boundary layer data obtained here are used in the trailing edge loss expression in order to gain a broad sense of the relative importance of the various parameters.

Using the base pressure and boundary layer parameters obtained from the CFD, the trailing edge loss was calculated from Equation 6.9, again noting that $C_{b}$ is defined differently than Denton's value of $C_{b, D}$. Figure 6.39 shows the trailing edge loss coefficient for the four cascades, as well as each individual term in Equation 6.9. While the results shown use the parameters obtained at a variety of Mach numbers, the expression developed by Denton assumes incompressible flow, as noted. The Mach number on the x-axis of Figure 6.39 indicates the mixed-out Mach number from the CFD case which was also used to obtain the base pressure and boundary layer parameters.

From Figure 6.39, the magnitudes of all three terms of Equation 6.9 are significant. The displacement thickness term remains roughly constant over the range of Mach numbers examined. The trailing edge thickness of the AF4 cascade is $1.22 \mathrm{~mm}$, and $1.26 \mathrm{~mm}$ for the three HS1 cascades. From Figure 6.37, the displacement thickness values are thus on the order of one tenth of the trailing edge thickness. Since the displacement thickness for all cascades is only a small fraction of the trailing edge thickness, the overall flow blockage at the trailing edge does not change significantly. For a blade with a thinner trailing edge, more variation 


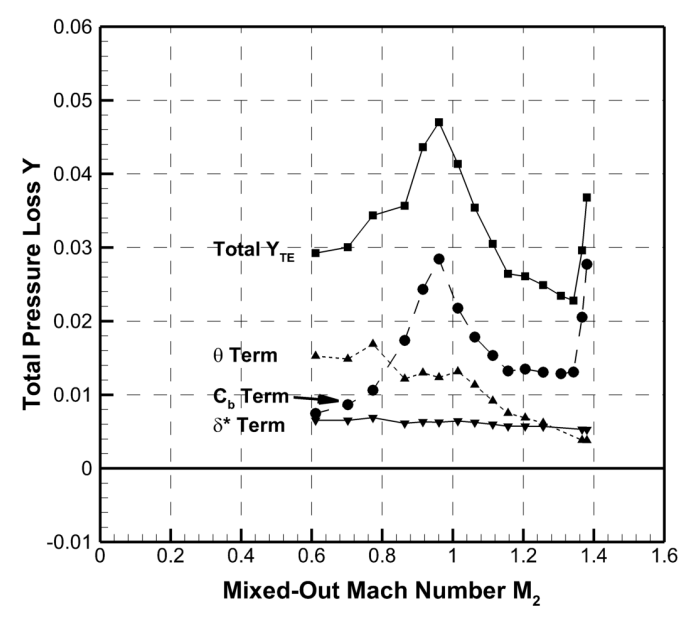

(a) AF4 Cascade

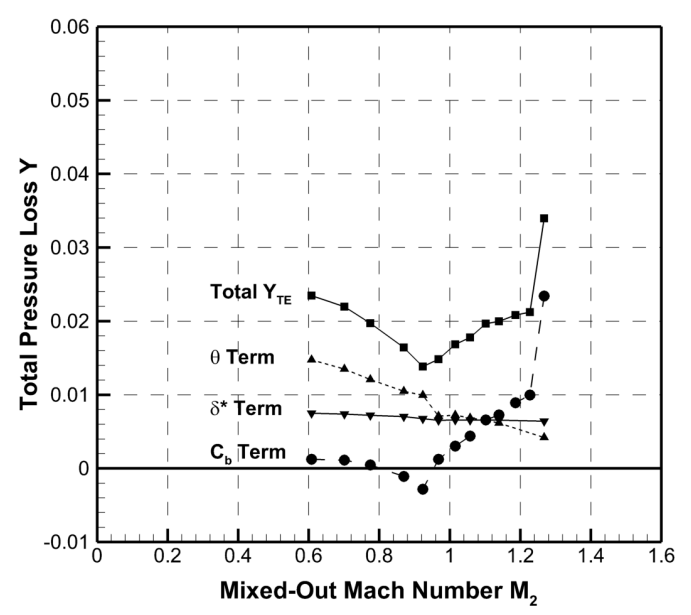

(c) HS1C Cascade

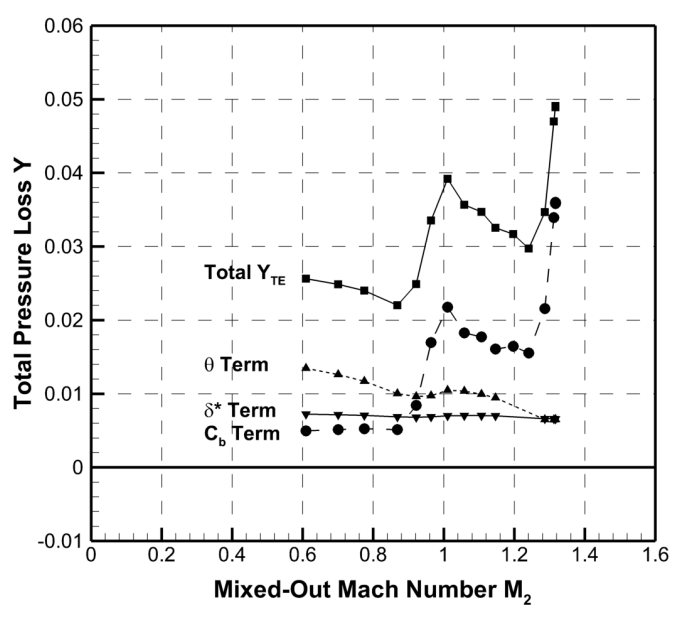

(b) HS1A Cascade

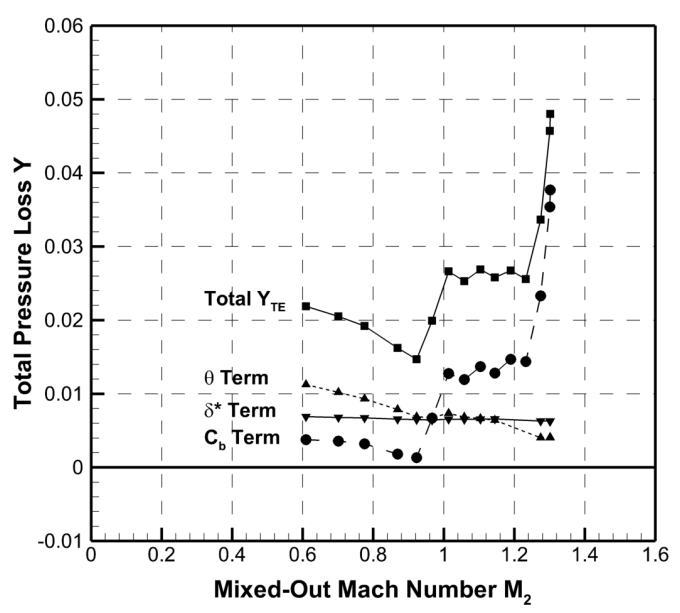

(d) HS1D Cascade

Figure 6.39: Trailing Edge Loss Prediction from Denton (1993) 
would occur in this displacement thickness term, particularly at lower Mach numbers where the displacement thickness is larger. The momentum thickness term tends to become less important at high Mach numbers, because the momentum thickness decreases as the Mach number is increased. The behaviour of the base pressure term is more easily understood by referring back to Figure 6.12. From the point of view of the linear momentum equation, the base pressure applies a force $($ Force $=$ Pressure $\times$ Area $)$ to the control volume. A negative $C_{b, D}$ value represents a net drag force whereas a positive value represents a thrust. For all cascades, the base pressure term becomes more important at transonic and supersonic Mach numbers. The abrupt increase of the base pressure term at high Mach numbers appears to cause the final rise in trailing edge losses. The exact influence of the base pressure is made ambiguous by the use of a different base pressure coefficient definition by Denton based on throat conditions. Using the continuity and momentum equations developed in Appendix B, the throat velocity and pressure can be estimated if density changes are ignored. Using such values, $C_{b, D}$ is estimated to be offset in the positive direction when compared with the values of $C_{b}$ obtained in this study. The influence of base pressure on the trailing edge losses may therefore be somewhat smaller than what is shown in Figure 6.39.

Figure 6.40 shows the incompressible trailing edge loss predictions from Denton (1993) compared with the overall profile losses previously presented in Figure 6.9. Both curves are taken from the numerical simulations. The overall profile losses are the sum of the boundary layer losses, the shock losses and the trailing edge losses. For this reason, it is difficult to accurately assess the prediction method without more information on the boundary layer and shock losses. Additional ambiguity is introduced by the different definitions of base pressure coefficient used between this study and that of Denton. Figure 6.40 does show that the general profile loss trends are reflected, and may even be driven by the trailing edge loss trends.

While a prediction of the shock losses was not made, the shock losses appear to increase with decreasing base pressure. This phenomenon was discussed in Section 6.8, when the shock losses were visible in the freestream portion of the wake profiles. It can be reasoned that the base pressure influences the strength of the trailing edge shocks by affecting the angle at which the suction and pressure side flows meet. Given that the base pressure appears to be 
correlated with the trailing edge losses and the shock losses, it can be concluded that accurate prediction of the base pressure is important for prediction of profile losses at transonic and supersonic Mach numbers.

\subsection{Applicability of the Denton (1993) Trailing Edge Loss Prediction to Compressible Flow}

The trailing edge loss expression developed by Denton assumes incompressible flow. In order to assess the applicability of Denton's approach for compressible flow, the control volume analysis was repeated in Appendix C without the incompressible flow assumption. Unsurprisingly, the result of the derivation is complex and requires an iterative procedure to solve the continuity, momentum and energy equations.

The trailing edge loss coefficient is defined more generally for the compressible flow as follows:

$$
Y_{T E}=\frac{P_{o, \text { Throat }}-P_{o 2}}{P_{o, \text { Throat }}-P_{\text {Throat }}}
$$

The throat was selected as a reference location for the total pressure loss coefficient in order to match Denton's expression. At the incompressible limit, the loss coefficient defined in Equation 6.11 simplifies to the form proposed by Denton:

$$
Y_{T E}=\frac{\Delta P_{o}}{0.5 \rho V_{\text {Throat }}^{2}}
$$

The trailing edge losses from the compressible analysis are shown in Figure 6.41, compared with the incompressible analysis. In order to isolate the effect of Mach number, all other parameters were held constant. The parameter values were the following, based on the lowest Mach number simulation of the AF4 cascade: $C_{b}=-0.111, \delta^{*}=0.246 \mathrm{~mm}$ and $\theta=0.138$ mm. From Figure 6.41, the Mach number is predicted to have a strong influence on the trailing edge losses. The baseline incompressible losses are nearly doubled at a throat Mach number of 1.0 . 


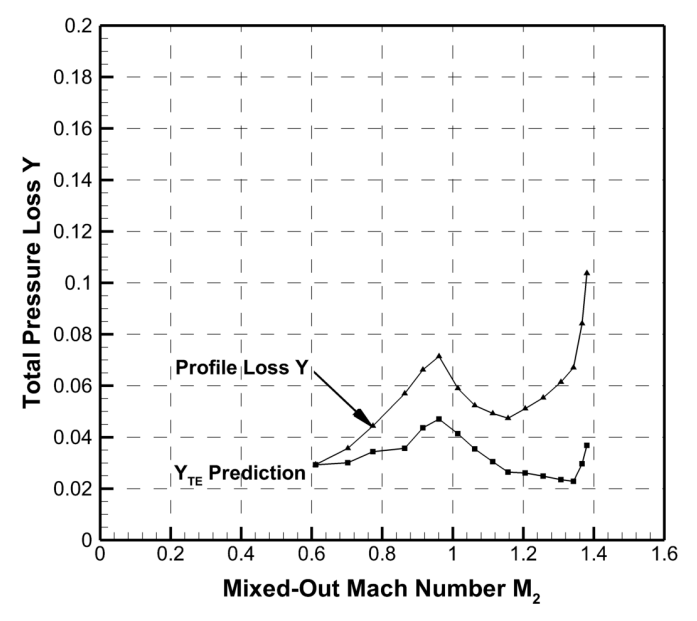

(a) AF4 Cascade

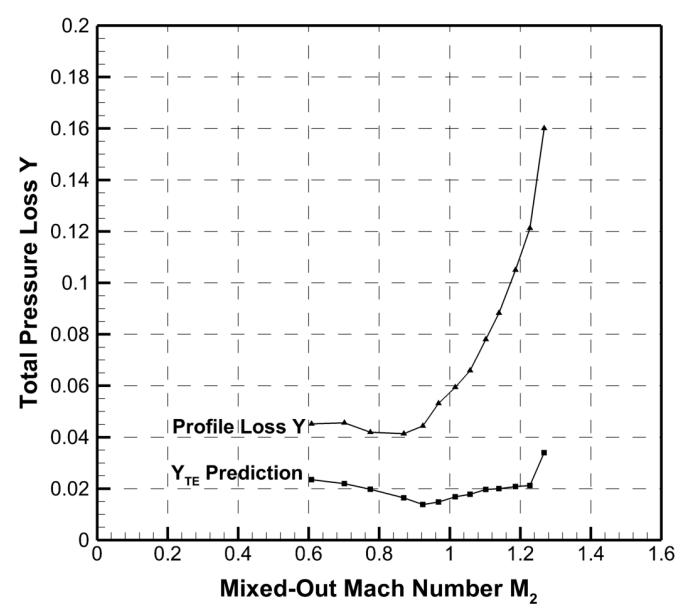

(c) HS1C Cascade

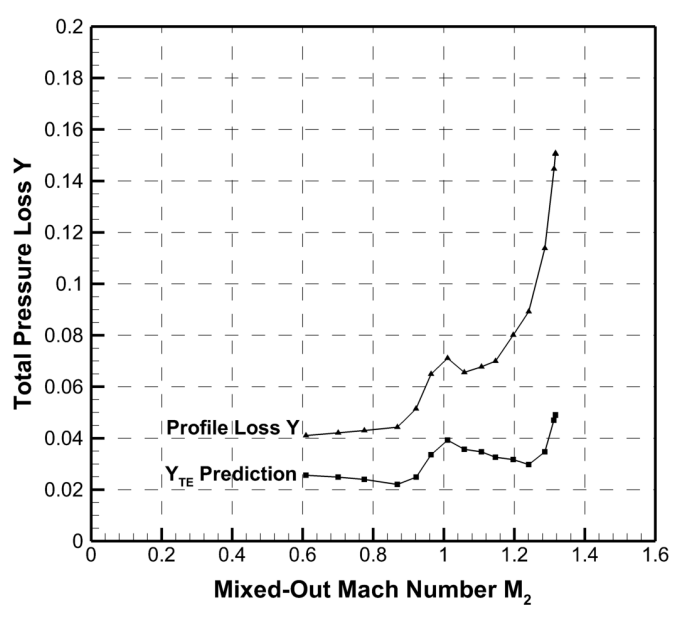

(b) HS1A Cascade

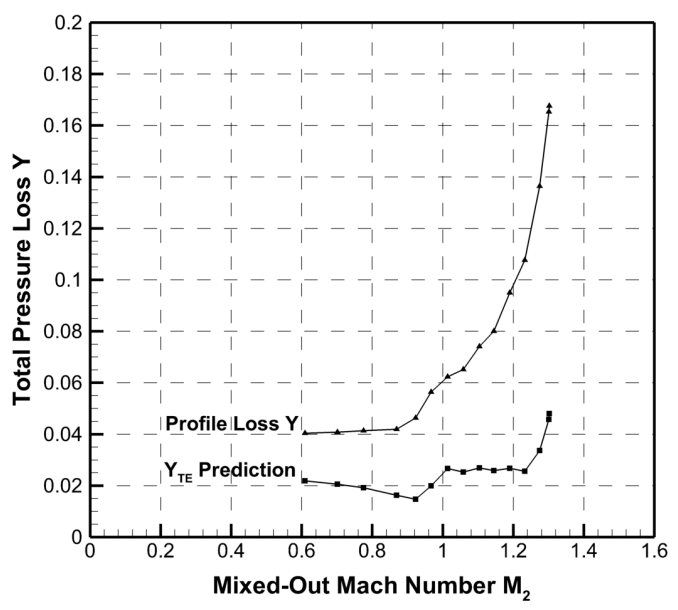

(d) HS1D Cascade

Figure 6.40: Trailing Edge Loss Prediction from Denton (1993) Compared to the Mixed-Out Profile Losses, Both Using Data from the Simulations 


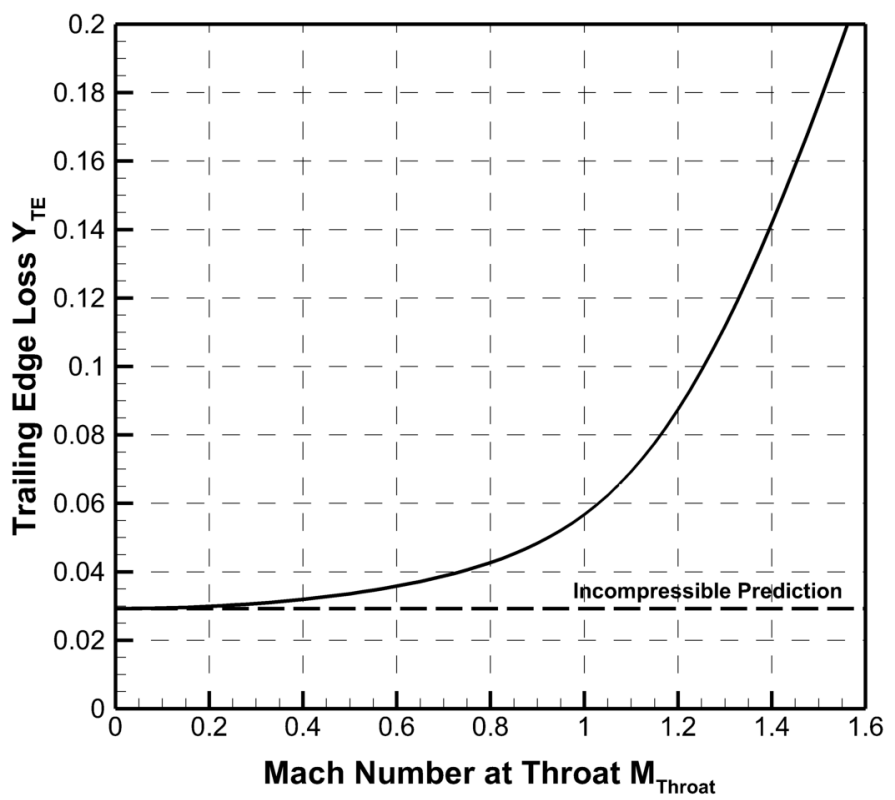

Figure 6.41: Effect of Mach Number on the Compressible Trailing Edge Loss Prediction of the AF4 Cascade, Using Constant Values of $C_{b}, \delta^{*}$ and $\theta$

Since the parameters are not actually constant at different Mach numbers, the calculation was repeated using modified parameters. In order to assess the sensitivity of the loss distribution to changes in the base pressure coefficient and boundary layer parameters, these parameters were varied by a factor of two. From Figure 6.37, the boundary layer displacement and momentum thicknesses both decrease as the Mach number is increased. The thicknesses in the present analysis were divided by two to reflect this decrease. From Figure 6.12, the base pressure coefficient becomes more negative as the Mach number is increased. The base pressure coefficient was thus multiplied by two. For each modified parameter, all other parameters were held at their original baseline values. The results of this sensitivity study are shown in Figure 6.42.

From Figure 6.42, the Mach number is the most important parameter affecting trailing edge losses. Variation of other parameters by a factor of two causes only a slight shift in the predicted losses. The effect of changing the base pressure coefficient $\left(C_{b, D}\right)$ and the momentum thickness $(\theta)$ diminishes as the Mach number is increased. Compared to the 


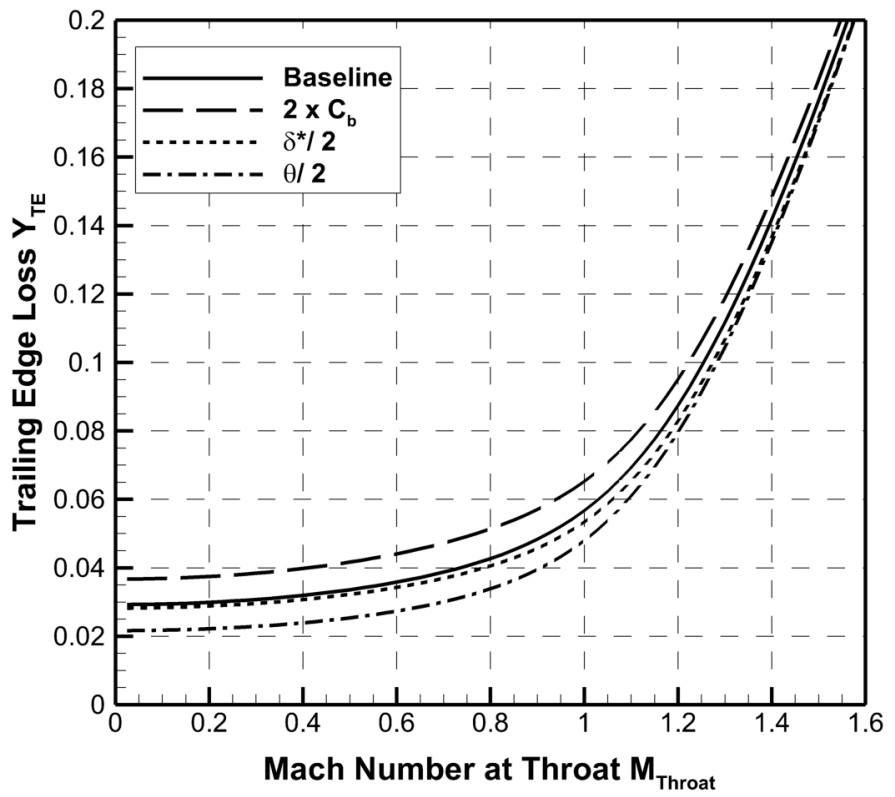

Figure 6.42: Effect of Individually Varying Each Parameter on the Trailing Edge Loss Prediction for Compressible Flow

other parameters, the effect of changing the displacement thickness $\left(\delta^{*}\right)$ remains very small, similar to the incompressible analysis shown in Figure 6.39. The influence of the displacement thickness is maximized near the sonic throat Mach number.

As the Mach number is increased, the increase in losses caused by the base pressure and the decrease in losses caused by the boundary layer thicknesses have competing effects. With all three parameters changing simultaneously as the Mach number is increased, the cumulative effect on the loss prediction would likely be small.

The Denton (1993) trailing edge loss expression is useful for making qualitative predictions of the trailing edge losses, as it was originally intended. The usefulness of the analysis begins to suffer when more quantitative results are desired. The trailing edge losses from the compressible prediction exceed even the overall measured profile losses at certain Mach numbers. This inaccuracy is no doubt due to the significant simplifying assumptions made during the derivation of the expression. 
All of the results for the present investigation have now been presented. The following chapter will summarize the most important conclusions from this work, as well as recommendations for future investigation. 


\section{Chapter 7}

\section{Conclusions and Recommendations}

\subsection{Conclusions}

The profile loss behaviour of four high-pressure turbine cascades has been investigated for flows in the transonic and supersonic Mach number range. Aerodynamic data on the AF4 cascade were collected in the high-speed wind tunnel at Carleton University, and were supplemented by data on the HS1 family of cascades, from Corriveau (2005). Numerical simulations were also performed in the current study for all four cascades. The objective of this study was to identify the mechanisms that determine the profile loss behaviour at transonic and supersonic Mach numbers.

From this study, it appears that the base pressure is strongly associated with the profile losses. It can be reasoned that the base pressure is related to the trailing edge losses by applying a pressure force on the fluid passing over the trailing edge, which would cause a momentum deficit in the wake of the blade. The base pressure might also have an influence on the shock losses by affecting the strength of the trailing edge shocks. At the trailing edge, lower base pressures would cause the suction side and pressure side flows to impinge upon each other at a higher angle, requiring stronger trailing edge shocks to realign the flows. The trailing edge shocks contribute to the profile losses outside of the wake.

An objective of this investigation was to better understand the flow mechanisms that cause the loss plateau at low supersonic outlet Mach numbers. A trailing edge loss prediction was made using a control volume analysis developed by Denton (1993), and using base 
pressure and boundary layer data obtained from the numerical simulations. This prediction method successfully recreated the loss plateau behaviour, and was driven primarily by the loss component resulting from the base pressure. For this reason, it appears that an accurate prediction of the base pressure is required in order to estimate the profile losses of turbine blades operating at low supersonic Mach numbers.

Given the importance of the base pressure, it was relevant to examine the usefulness of the Sieverding et al. (1979) base pressure correlation. This correlation predicts the base pressure over a range of isentropic Mach numbers, and is based on the unguided turning angle $\left(\theta_{u}\right)$ and the trailing edge wedge angle $\left(W_{e}\right)$. This correlation was able to predict the base pressure in general terms but did not fully capture the behaviour, suggesting that important parameters are missing. It seemed that the base pressure was influenced by the shape factor of the suction surface boundary layer, just upstream of the trailing edge. Lower shape factors, representing fuller boundary layer velocity distributions, were assumed to generate greater flow entrainment over the base region, resulting in lower base pressures. In order to aid in preliminary design of turbine blades, it is desirable to have a base pressure correlation which is based on simple geometric parameters. The suction surface boundary layer just upstream of the trailing edge is affected by streamwise pressure gradients along the length of the blade surface, and by the shock-boundary layer interaction. A simple method of predicting the boundary layer shape factor upstream of the trailing edge or the base pressure remains elusive.

From the blade-to-blade contour plots of Mach number, the upper end of the loss plateau occurs when the trailing edge shocks reach the trailing edge of the adjacent blade. This flow condition is associated with a decrease in base pressure and an increase in profile losses. It is believed that the flow acceleration caused by the expansion fan on the adjacent trailing edge thins the boundary layer, allowing greater flow entrainment to occur at the trailing edge, leading to a lower base pressure. It is advised that turbine blades be designed for a Mach number below this condition in order to avoid a high profile loss penalty. 


\subsection{Recommendations}

In order to estimate the profile losses of a turbine blade at transonic and supersonic Mach numbers, it appears that an accurate prediction of the base pressure is required. This prediction would be most useful if it was based on a limited number of geometric and flow parameters. The current research suggests that the boundary layer, and in particular the shape factor, have an influence on the base pressure. A future investigation might focus on better understanding how the shock-boundary layer interaction affects the boundary layer. Specifically, it is hypothesized that the unguided turning affects the angle at which the trailing edge shock impinges on the suction surface. The impingement angle of the shock may affect how much the boundary layer is altered by the shock-boundary layer interaction. In order to investigate this relationship further, one might begin by quantifying the strength of the trailing edge shocks (by measuring the pressure ratio across the shocks, for example). The boundary layer could then be compared upstream and downstream of the shock-boundary layer interaction, while taking into consideration the angle of the shock impingement.

The blades investigated here had very similar trailing edge thicknesses. The effect of the trailing edge blockage on the base pressure and profile losses was not investigated, though the base pressure is likely a function of the trailing edge blockage.

Once the influence of these parameters is better understood, it may become possible to modify the existing empirical loss correlations in order to better predict the profile losses at

high Mach numbers. The modification would likely take the form of a new Mach number correction that could replace the CFM correction used in the Kacker \& Okapuu (1982) loss correlation. 


\section{References}

(2013). ANSYS CFX-Solver Modeling Guide. ANSYS Inc., release 15.0 edition.

Ainley, D. G. \& Mathieson, G. (1951). A method of performance estimation for axial-flow turbines. Aeronautical Research Council of Great Britain, R\&M 2974.

Baines, N. C., King, P. I., Oldfield, M. L. G., Kiock, R., Hoheisel, H., Ramm, G., Lehthaus, F., Kost, F. H., \& Sieverding, C. H. (1986). A Comparison of Aerodynamic Measurements of the Transonic Flow Through a Plane Turbine Cascade in Four European Wind Tunnels. Technical Report Report No. OUEL 1624/86, Oxford University.

Baljé, O. E. \& Binsley, R. L. (1968). Axial turbine performance evaluation. ASME Paper $68-G T-13$, .

Binder, A., Schroeder, T., \& Hourmouziadis, J. (1989). Turbulence measurements in a multistage low-pressure turbine. ASME Journal of Turbomachinery, Vol. 111, pp. 153-161.

Bolte, W. (1964). Zur berechnung and optimiterung des wirkungsgrades axialer stromungsmaschinen. VDI ForschHftscheft 501.

Chen, S. (1987). A loss model for the transonic flow low-pressure steam turbine blades. Institute of Mechanical Engineers Paper No. C269/87, (C269/87).

Corriveau, D. (2005). Influence of loading distribution on the performance of high pressure turbine blades. Ph.D. thesis, Carleton University. 
Corriveau, D. \& Sjolander, S. A. (2007). Influence of loading distribution on the off-design performance of high-pressure turbine blades. ASME Journal of Turbomachinery, Vol. 129, pp. 563-571.

Craig, H. R. M. \& Cox, H. J. A. (1971). Performance estimation of axial flow turbines. Proceedings of the Institute of Mechanical Engineers, Vol. 185 32/71, pp. 407-424.

Cumpsty, N. A. \& Horlock, J. H. (2006). Averaging nonuniform flow for a purpose. ASME Journal of Turbomachinery, Vol. 128, pp. 120-129.

Deich, M. E. (1953). Flow of gas through turbine lattices. NACA Tech. Memo. 1393.

Delery, J. \& Marvin, J. (1986). Shock-wave boundary layer interactions. AGARDograph, 280.

Denton, J. D. (1971). A method of predicting the profile loss of turbine cascades. Aeronaut. Res. Counc, 33222 Turbo. 176 (Unpublished).

Denton, J. D. (1973). A survey and comparison of methods for predicting the profile loss of turbine blades. Institute of Mechanical Engineers Paper No. C76/73.

Denton, J. D. (1993). Loss mechanisms in turbomachines. ASME Journal of Turbomachinery, Vol. 115, pp. 621-656.

Denton, J. D. \& Xu, L. (1990). The trailing edge loss of transonic turbine blades. ASME Journal of Turbomachinery, Vol. 112, pp. 277-285.

Detemple-Laake, E. (1989). Measurement of the flow field in the blade passage and side-wall resgion of a plane turbine cascade. AGARD-CP-469.

Dunham, J. \& Came, P. M. (1970). Improvements to the ainley-mathieson method of turbine performance prediction. ASME Journal of Engineering for Gas Turbines and Power, Vol. 92 Ser A, pp. 252-256.

Graham, C. G. \& Kost, F. H. (1979). Shock boundary layer interaction on high turning transonic turbine cascades. ASME Paper 79-GT-3\%. 
Granovskiy, A., Kostege, M., \& Vassiliev, V. (2014). Effect of unguided turning angle and trailing edge shape on cooled blade loss. Proceedings of the ASME Turbo Expo.

Hall, S. R. (2012). Contributions to High-Speed Linear Turbine Cascade Testing: Design of a Wind Tunnel Control System; and Investigation of the Effects of Compressibility on Total Pressure Losses. M.A.Sc. thesis, Carleton University.

Hoheisel, H., Kiock, R., Lichtfuss, H. J., \& Fottner, L. (1987). Influence of free-stream turbulence and blade pressure gradient on boundary layer and loss behavior of turbine cascades. ASME Journal of Turbomachinery, Vol. 109, pp. 210-219.

Hourmouziadis, J. (1989). Aerodynamic design of low pressure turbines. AGARD-LS-16\%.

Islam, A. M. T. (1999). An Experimental and Computational Study of the Aerodynamics of Turbine Blades With Damage. Ph.D. thesis, Carleton University.

Jeffries, M. S. (1994). The Commissioning and Enhancement of a Blow Down Wind Tunnel. M.A.Sc. thesis, Carleton University.

Jeffries, M. S. (2000). Initial Investigations of Transonic Turbine Aerodynamics using the Carleton University High-Speed Wind Tunnel. Ph.D. thesis, Carleton University.

Jeffries, M. S., Jouini, D., \& Sjolander, S. A. (1997). Determining sampling rates and times in a high speed wind tunnel. CASI 6th Symposium on Aerodynamics, Toronto.

Jouini, D. B. M. (2000). Experimental Investigation of Two Transonic Linear Turbine Cascades at Off-Design Conditions. Ph.D. thesis, Carleton University.

Jouini, D. B. M., Sjolander, S. A., \& Moustapha, S. H. (2002). Midspan flow-field measurements for two transonic linear turbine cascades at off-design conditions. ASME Journal of Turbomachinery, Vol. 124, pp. 176-186.

Kacker, S. C. \& Okapuu, U. (1982). A mean line prediction method for axial flow turbine efficiency. ASME Journal of Engineering for Gas Turbines and Power, Vol. 104, pp. 111119. 
Kiock, R., Lehthaus, F., Baines, N. C., \& Sieverding, C. H. (1986). The transonic flow through a plane turbine cascade as measured in four european wind tunnels. Journal of Engineering for Gas Turbines and Power, 108, 277-284.

Ladwig, M. \& Fottner, L. (1993). Experimental investigations of the influence of incoming wakes on the losses of a linear turbine cascade. ASME Paper 93-GT-394.

Langtry, R. B. \& Mentor, F. R. (2005). Transition modmodel for general cfd applications in aeronautics. AIAA 2005-522.

Li, S.-M., Chu, T.-L., Yoo, Y.-S., \& Ng, W. F. (2004). Transonic and low supersonic flow losses of two steam turbine blades at large incidences. Journal of Fluids Engineering, 126, 966-975.

Mayle, R. E. (1991). The role of laminar-turbulent transition in gas turbine engines. ASME Journal of Turbomachinery, Vol. 113, pp. 509 - 537.

Mee, D. J., Baines, N. C., Oldfield, M. L. G., \& Dickens, T. E. (1992). An examination of the contributions to a loss on a transonic turbine in cascade. ASME Journal of Turbomachinery, Vol. 114, pp. 155-162.

Porreca, L., Hollenstein, M., Kalfas, A. I., \& Abhari, R. S. (2007). Turbulence measurements and analysis in a multistage axial turbine. Journal of Propulstion and Power, 23(1).

Prassad, A. (2005). Calculation of the mixed-out state in turbomachine flows. ASME Journal of Turbomachinery, 127, 564-572.

Rodger, P., Sjolander, S. A., \& Moustapha, S. H. (1992). Establishing two-dimensional flow in a large-scale planar turbine cascade. AIAA Paper 92-3066.

Sieverding, C. H. (1985). Thermodynamics and Fluid Mechanics of Turbomachinery Volume II, chapter Axial Turbine Performance Prediction Methods, (pp. 737-784). Martinus Nijhoff Publishers: Dordrecht. 
Sieverding, C. H., Stanislis, M., \& Snoek, J. (1979). The base pressure problem in transonic cascades. ASME Paper 79-GT-120.

Sooriyakumaran, C. (2014). Experimental Study of Profile Losses in Three Transonic Turbine Cascades. M.A.Sc. thesis, Carleton University.

Taremi, F., Sjolander, S. A., \& Abo El Ella, H. M. (2008). Extending the capabilities of a high-speed wind tunnel to secondary flow measurements in transonic linear turbine cascades. AIAA Paper No. 5272.

Tavoularis, S. (2005). Measurement in Fluid Mechanics. Cambridge University Press.

Traupel (1966). Thermische Turbomaschinen. Berlin: Springer-Verlag. (As referenced by Denton (1973)).

White, F. M. (2006). Fluid Mechanics. McGraw-Hill, 6th edition.

Xu, L. \& Denton, J. D. (1988). The base pressure and loss of a family of four turbine blades. Journal of Turbomachinery, 110, 9-17. 


\section{Appendix A}

\section{Upstream Reference Total Pressure Calibration}

As described in Section 4.3.1, a reference total pressure is measured far upstream of the cascade such that the wake of the pitot tube is mostly dissipated by the time the flow reaches the cascade. The total pressure measurement must therefore be corrected for losses that occur in the inlet duct. Prior to the current study, the common practice was to apply the following correction factor to the total pressure measurement:

$$
\frac{P_{o 1}}{P_{o, r e f}}=0.995
$$

where $P_{o, r e f}$ is the measured reference total pressure far upstream, and $P_{o 1}$ is the total pressure corrected for a location just upstream of the cascade. The correction factor is a constant, and is thus independent of the Mach number. This correction was based on total pressure measurements by Corriveau (2005), using a pitot tube placed $40 \mathrm{~mm}$ upstream of the cascade.

The upstream reference total pressure calibration was revisited in the current investigation when the profile losses obtained using this calibration were unusually low. A pitot tube, shown schematically in Figure A.1, was aligned with the flow at midspan, $60 \mathrm{~mm}$ upstream of the leading edge of the AF4 cascade. The wind tunnel was then operated over a wide range of outlet isentropic Mach numbers, and the pitot tube measurements were compared to the upstream reference total pressure measurements. The results of this experiment are

shown in Figure A.2. In all cases, the $\frac{P_{o 1}}{P_{o, r e f}}$ ratio is higher than the expected value of 0.995 . Furthermore, the ratio varies with Mach number . In order to better capture the behaviour of 


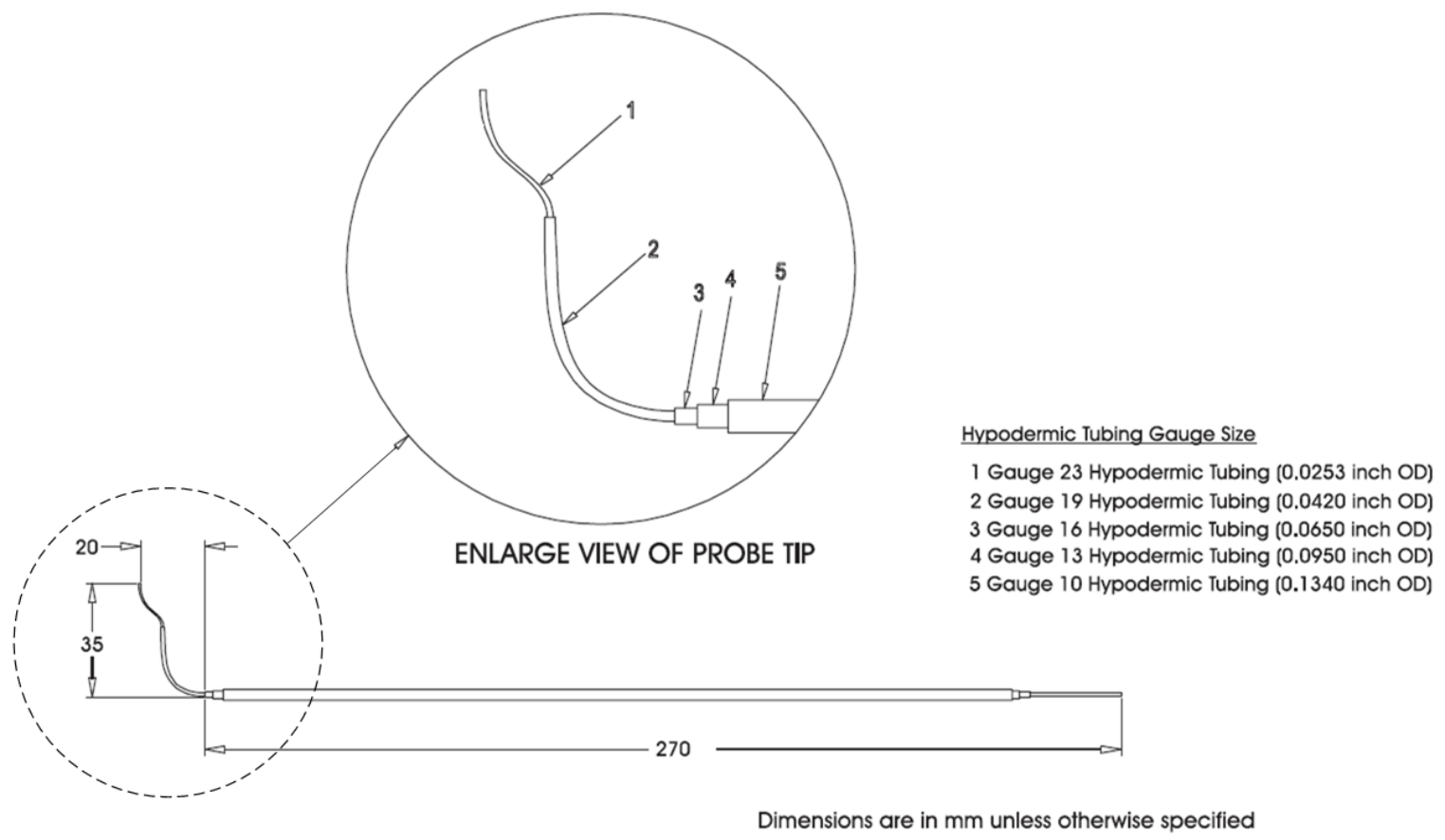

Figure A.1: Pitot tube used for upstream total pressure measurements

the inlet duct losses, the following fourth-order polynomial curve fit was applied to the data:

$$
\frac{P_{o 1}}{P_{o, \text { ref }}}=A\left(M_{2, i s}\right)^{4}+B\left(M_{2, i s}\right)^{3}+C\left(M_{2, i s}\right)^{2}+D\left(M_{2, i s}\right)+E
$$

where the polynomial coefficients have the following values:

$$
\begin{aligned}
& A=0.00814343406050 \\
& B=-0.0206607779426 \\
& C=0.0214265096391 \\
& D=-0.0151406806643 \\
& E=1.00199928100
\end{aligned}
$$

The correlation coefficient for this curve fit is $r=0.9444$. It was found that increasing the order of the polynomial curve fit resulted in negligible increase in the correlation coefficient, so the fourth-order polynomial was selected. The calibration process using the pitot tube should 


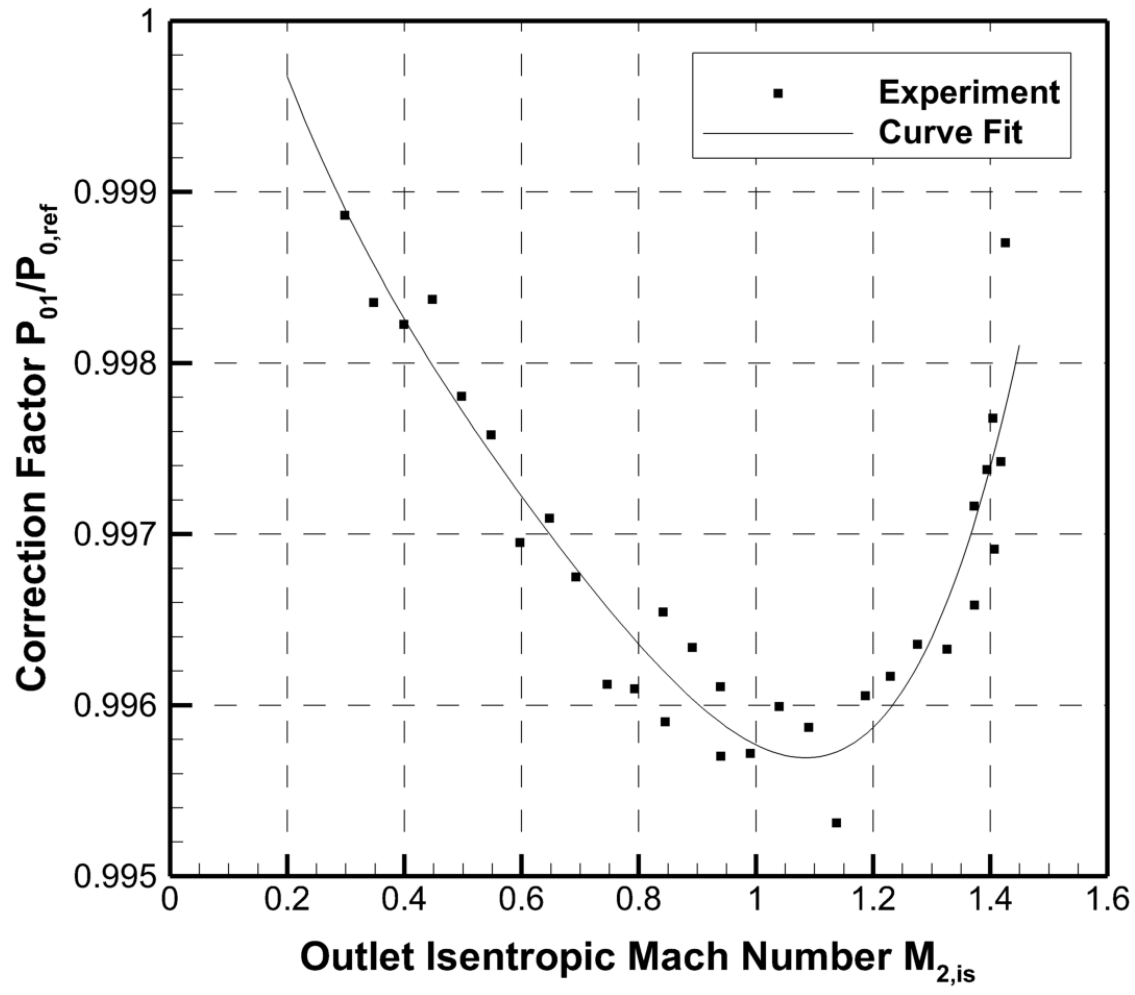

Figure A.2: 4th order polynomial curve fit of the correction factor for inlet total pressure be repeated for every new cascade that is installed in the wind tunnel, since the relationship between the upstream losses and downstream Mach number likely depends on the geometry of the cascade. 


\section{Appendix B}

\section{Incompressible Control Volume Analysis for the Trailing Edge Losses}

\section{B.1 Introduction}

As part of a comprehensive review of turbomachinery losses, Denton (1993) uses a control volume analysis to derive a simple expression for trailing edge losses. Some steps of the derivation were omitted by Denton, so the full derivation is presented here in order to clarify the simplifying assumptions that were applied.

Denton (1993) proposes a control volume just downstream of two turbine blades with constant thickness and no unguided turning, as shown in Figure B.1. The idealization shown is for blades with a trailing edge thickness $t$, a stagger angle $\alpha$ and a blade pitch $(w / \cos \alpha)$.

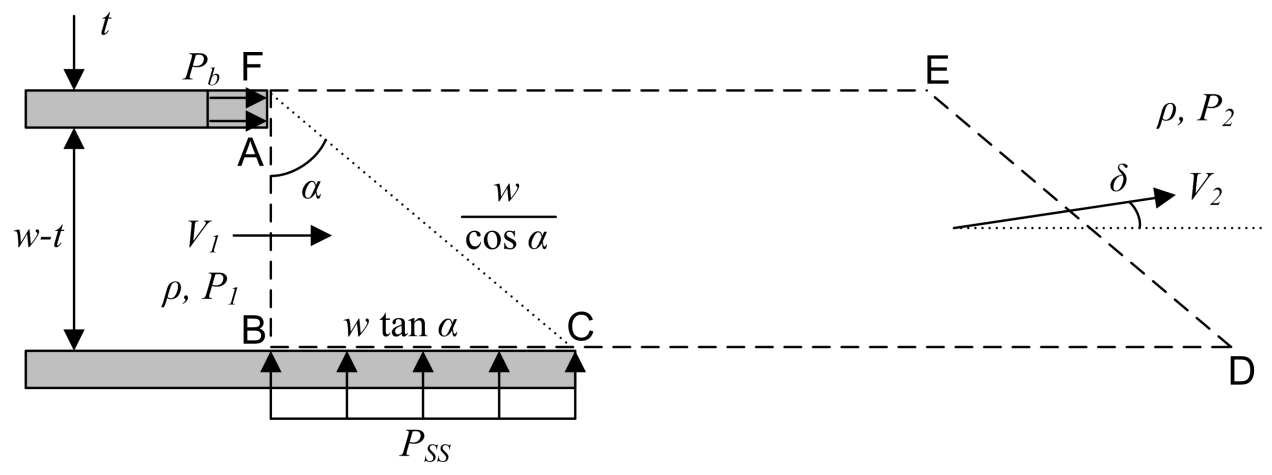

Figure B.1: Control Volume Defined Downstream of the Trailing Edge of a Row of Staggered Blades in Incompressible Flow (Adapted from Denton, 1993) 
The flow is considered uniform at the throat $\mathrm{AB}$ and also far downstream, where viscous dissipation has removed all flow non-uniformities. The base pressure $P_{b}$ is applied across the flat trailing edge surface of the upper blade. A uniform suction surface pressure $P_{S S}$ is applied along the suction surface of the lower blade. The boundary layer displacement thickness $\left(\delta^{*}\right)$ and momentum thickness $(\theta)$ are considered to be the combined values for the suction-side and pressure-side boundary layers. Incompressible flow is assumed, allowing for a simplified solution. Denton then writes the continuity and $\mathrm{x}$-momentum equations for this control volume in order to arrive at an expression for the trailing edge loss coefficient.

The reader should be aware that the nomenclature used in this appendix differs from the rest of the report. In the current derivation, the subscript 1 refers to the plane at the blade throat, which is the control volume inlet. In the rest of the report, the subscript 1 refers to the inlet of the cascade.

\section{B.2 Continuity Equation}

Based on the control volume defined in Figure B.1, the continuity equation can be written on the basis of mass flow rate per unit span, as follows:

$$
\left(\frac{\dot{m}}{\operatorname{span}}\right)_{1}=\left(\frac{\dot{m}}{\operatorname{span}}\right)_{2}
$$

where the subscripts 1 and 2 denote the conditions across the control volume inlet $\mathrm{AB}$ and outlet DE, respectively. The mass flow rate for uniform incompressible flow is $\dot{m}=\rho V A$, so Equation B.1 can be rewritten as:

$$
\rho V_{1}\left(w-t-\delta^{*}\right)=\rho V_{2} \cos (\alpha-\delta) \frac{w}{\cos \alpha}
$$

In order to simplify the right-hand side of Equation B.2, the following trigonometric identity is applied:

$$
\cos (\alpha-\delta)=\cos \alpha \cos \delta-\sin \alpha \sin \delta
$$


Assuming that the flow deviation angle $\delta$ is small, it can be assumed that $(\cos \delta \simeq 1)$ and $(\sin \delta \simeq \delta)$ where $\delta$ is expressed in radians. The trigonometric identity can be rewritten as:

$$
\cos (\alpha-\delta)=\cos \alpha(1)-\delta \sin \alpha
$$

Substituting this identity into the right-hand side of Equation B.2 yields the following:

$$
\rho V_{1}\left(w-t-\delta^{*}\right)=\rho V_{2} w \frac{\cos \alpha-\delta \sin \alpha}{\cos \alpha}
$$

and simplifying this result yields the following:

$$
\rho V_{1}\left(w-t-\delta^{*}\right)=\rho V_{2} w(1+\delta \tan \alpha)
$$

In order to obtain a value for the flow deviation angle $\delta$, Denton applies the momentum equation in the vertical direction. However, the final expression for $\delta$ is a function of the suction surface pressure $P_{S S}$. Without additional information, a value cannot be assigned to the suction surface pressure. Furthermore, substituting the final expression for $\delta$ into Equation B.6 yields an algebraically complex result. Instead, Denton assumes that the flow deviation angle $\delta=0$ for simplicity. Since the flow is assumed incompressible, the density $\rho$ can also be canceled out, as follows:

$$
\not V_{1}\left(w-t-\delta^{*}\right)=\phi V_{2} w(1+\delta \cos \alpha)^{0}
$$

Rearranging Equation B.7, an expression for the ratio of outlet to inlet velocity can be obtained, as follows:

$$
\frac{V_{2}}{V_{1}}=\frac{w-t-\delta^{*}}{w}
$$




\section{B.3 Momentum Equation}

Denton writes the momentum equation in the horizontal direction in the following form:

$$
\sum(\mathrm{x}-\text { Momentum })_{1}=\sum(\mathrm{x}-\text { Momentum })_{2}
$$

Expanding this expression yields the following:

$$
(w-t) P_{1}+t P_{b}+\dot{m}_{1} V_{1}-\rho V_{1}^{2} \theta=w P_{2}+\dot{m}_{2} V_{2} \cos \delta
$$

where the term containing $\theta$ is the momentum reduction as a result of the boundary layer momentum thickness. Substituting the inlet and outlet mass flow rates $\left(\dot{m}_{1}\right.$ and $\left.\dot{m}_{2}\right)$ from Equation B.6 into the momentum equation and setting the deviation angle $\delta \simeq 0$, the following is obtained:

$$
(w-t) P_{1}+t P_{b}+\left(\rho V_{1}\left(w-t-\delta^{*}\right)\right) V_{1}-\rho V_{1}^{2} \theta=w P_{2}+\left(\rho V_{2} w\right) V_{2}
$$

Dividing Equation B.11 by $w$, the expression can be rearranged as follows:

$$
P_{1}-P_{2}=-\rho V_{1}^{2}\left(1-\frac{t}{w}-\frac{\delta^{*}}{w}-\frac{\theta}{w}\right)+\rho V_{2}^{2}+\left(P_{1}-P_{b}\right) \frac{t}{w}
$$

For incompressible flow, the static pressures $P_{1}$ and $P_{2}$ can be expressed in terms of the total and dynamic pressures, as shown:

$$
\begin{aligned}
& P_{1}=P_{01}-\frac{1}{2} \rho V_{1}^{2} \\
& P_{2}=P_{02}-\frac{1}{2} \rho V_{2}^{2}
\end{aligned}
$$

Substituting these relations for $P_{1}$ and $P_{2}$ in Equation B.12 yields the following:

$$
P_{01}-P_{02}=\frac{1}{2} \rho V_{1}^{2}-\frac{1}{2} \rho V_{2}^{2}-\rho V_{1}^{2}\left(1-\frac{t}{w}-\frac{\delta^{*}}{w}-\frac{\theta}{w}\right)+\rho V_{2}^{2}+\left(P_{1}-P_{b}\right) \frac{t}{w}
$$


Rearranging, the following is obtained:

$$
P_{01}-P_{02}=-\frac{1}{2} \rho V_{1}^{2}\left(1-2\left(\frac{t}{w}+\frac{\delta^{*}}{w}+\frac{\theta}{w}\right)\right)+\frac{1}{2} \rho V_{2}^{2}+\left(P_{1}-P_{b}\right) \frac{t}{w}
$$

Dividing Equation B.15 by $\frac{1}{2} \rho V_{1}^{2}$ yields the following expression:

$$
\frac{P_{01}-P_{02}}{\frac{1}{2} \rho V_{1}^{2}}=\frac{V_{2}^{2}}{V_{1}^{2}}-1+2\left(\frac{t}{w}+\frac{\delta^{*}}{w}+\frac{\theta}{w}\right)-\frac{P_{b}-P_{1}}{\frac{1}{2} \rho V_{1}^{2}} \frac{t}{w}
$$

The last term in Equation B.16 can be rewritten using the base pressure coefficient $\left(C_{b, D}\right)$, which is defined by Denton as follows:

$$
C_{b, D}=\frac{P_{b}-P_{1}}{\frac{1}{2} \rho V_{1}^{2}}
$$

Denton chose the conditions at the throat (at point 1) as reference conditions for the coefficient, since the algebra in the derivation works out to a compact expression. However, the base pressure coefficient is typically defined based on the downstream conditions (at point 2).

Equation B.8, developed from the continuity equation, can now be substituted into Equation B.16, in addition to the base pressure coefficient $C_{b, D}$, as follows:

$$
\frac{P_{01}-P_{02}}{\frac{1}{2} \rho V_{1}^{2}}=\left(\frac{w-t-\delta^{*}}{w}\right)^{2}-1+2\left(\frac{t}{w}+\frac{\delta^{*}}{w}+\frac{\theta}{w}\right)-C_{b, D} \frac{t}{w}
$$

Expanding the term that is raised to the second power allows many of terms to be canceled, as shown:

$$
\frac{P_{01}-P_{02}}{\frac{1}{2} \rho V_{1}^{2}}=\left(1-2\left(\frac{t+\delta^{*}}{w}\right)+\left(\frac{t+\delta^{*}}{w}\right)^{2}\right)-\not 1+2\left(\frac{t+\delta^{*}}{w}\right)+\frac{2 \theta}{w}-C_{b, D} \frac{t}{w}
$$

Denton's expression for the trailing edge total pressure losses is thus the following:

$$
\zeta=\frac{P_{01}-P_{02}}{\frac{1}{2} \rho V_{1}^{2}}=-\frac{C_{b, D} t}{w}+\frac{2 \theta}{w}+\left(\frac{\delta^{*}+t}{w}\right)^{2}
$$




\section{Appendix C}

\section{Compressible Control Volume Analysis for the Trailing Edge Losses}

\section{C.1 Introduction}

In order to assess the applicability of the Denton (1993) trailing edge loss expression in compressible flow, the derivation performed in Appendix B is repeated without the incompressible flow assumption. The modified control volume is shown in Figure C.1. This control volume greatly resembles the one shown in Appendix B, with the addition of changing density and Mach number.

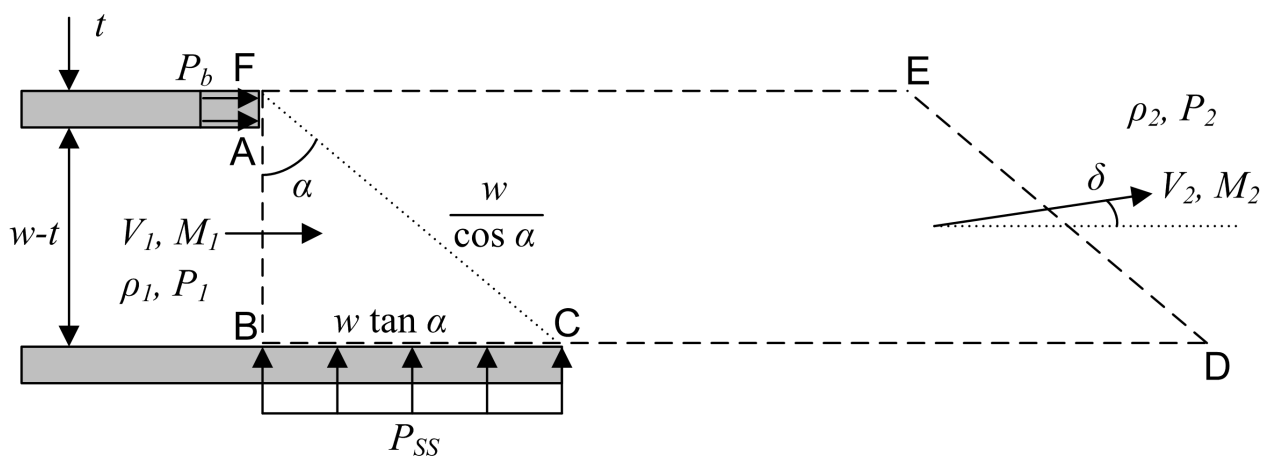

Figure C.1: Control Volume Defined Downstream of the Trailing Edge of a Row of Staggered Blades in Compressible Flow (Adapted from Denton, 1993) 
The total pressure loss coefficient for the trailing edge losses is defined as follows, based on the control volume in Figure C.1.

$$
Y_{T E}=\frac{P_{01}-P_{02}}{P_{01}-P_{1}}
$$

The reference conditions for the coefficient are defined at the throat plane (1). When the flow is incompressible, the denominator $P_{01}-P_{1}$ becomes $0.5 \rho_{1} V_{1}^{2}$, so the loss coefficient defined here is equivalent to the one defined by Denton (1993) in the incompressible derivation.

The following isentropic Mach number relationship can be used to rewrite the trailing edge loss coefficient:

$$
P_{0}=P\left(1+\frac{\gamma-1}{2} M^{2}\right)^{\frac{\gamma}{\gamma-1}}
$$

Inserting Equation C.2 into the trailing edge loss coefficient and dividing through by $P_{1}$ yields the following expression:

$$
Y_{T E}=\frac{\left(1+\frac{\gamma-1}{2} M_{1}^{2}\right)^{\frac{\gamma}{\gamma-1}}-\frac{P_{2}}{P_{1}}\left(1+\frac{\gamma-1}{2} M_{2}^{2}\right)^{\frac{\gamma}{\gamma-1}}}{\left(1+\frac{\gamma-1}{2} M_{2}^{2}\right)^{\frac{\gamma}{\gamma-1}}-1}
$$

The trailing edge loss coefficient is now in a form that requires only a static pressure ratio and the Mach numbers at the throat plane and the mixed-out plane. The following sections will demonstrate how the continuity, momentum and energy equations can be used to obtain these values.

\section{C.2 Continuity Equation}

The continuity equation can be defined on the basis of mass flow rate per unit span, similar to the incompressible derivation. However, in this case the density is allowed to vary from the throat (point 1) to the mixed-out plane (point 2).

$$
\rho_{1} V_{1}\left(w-t-\delta^{*}\right)=\rho_{2} V_{2} \cos (\alpha-\delta) \frac{w}{\cos \alpha}
$$


As with the incompressible case, the flow deviation angle $\delta$ is assumed to be zero. Equation C.4 simplifies as follows:

$$
\rho_{1} V_{1}\left(w-t-\delta^{*}\right)=\rho_{2} V_{2} w
$$

Since the flow is compressible, it is more appropriate to work with the Mach number than velocity. Velocity is related to the Mach number through the speed of sound $(a)$ through the following expression: $V=M a$. The speed of sound is defined as $a=\sqrt{\gamma R T}$, where $\gamma$ is the specific heat ratio, $R$ is the ideal gas constant for air and $T$ is the temperature. Furthermore, the speed of sound definition can be modified by substituting the ideal gas relationship $P=\rho R T$. Combining these expressions leads to the following expression for velocity

$$
V=M \sqrt{\frac{\gamma P}{\rho}}
$$

Substituting Equation C.6 for the velocity into the continuity equation leads to the following result:

$$
M_{1} \sqrt{\gamma \rho_{1} P_{1}}\left(w-t-\delta^{*}\right)=M_{2} \sqrt{\gamma \rho_{2} P_{2}} w
$$

Raising Equation C.7 to the second power eliminates the square roots.

$$
M_{1}^{2} \gamma \rho_{1} P_{1}\left(w-t-\delta^{*}\right)^{2}=M_{2}^{2} \gamma \rho_{2} P_{2} w^{2}
$$

Equation C.8 can then be rearranged to obtain an expression for the ratio of throat to mixedout density, as follows:

$$
\frac{\rho_{2}}{\rho_{1}}=\frac{P_{1}}{P_{2}} \frac{M_{1}^{2}}{M_{2}^{2}} \frac{\left(w-t-\delta^{*}\right)^{2}}{w^{2}}
$$

\section{C.3 Momentum Equation}

As with the incompressible analysis, the momentum equation in the horizontal direction can be written as follows:

$$
(w-t) P_{1}+t P_{b}+\dot{m}_{1} V_{1}-\rho_{1} V_{1}^{2} \theta=w P_{2}+\dot{m}_{2} V_{2} \cos \delta
$$


where the term containing $\theta$ is the momentum reduction as a result of the boundary layer momentum thickness. Substituting the inlet and outlet mass flow rates $\left(\dot{m}_{1}\right.$ and $\left.\dot{m}_{2}\right)$ from Equation C.7 into the momentum equation and setting the deviation angle $\delta \simeq 0$, the following is obtained:

$$
(w-t) P_{1}+t P_{b}+\left(\rho_{1} V_{1}\left(w-t-\delta^{*}\right)\right) V_{1}-\rho_{1} V_{1}^{2} \theta=w P_{2}+\left(\rho_{2} V_{2} w\right) V_{2}
$$

Rearranging Equation C.11, the following can be obtained:

$$
\rho_{1} V_{1}^{2}\left(w-t-\delta^{*}-\theta\right)-\rho_{2} V_{2}^{2} w=\left(P_{1}-P_{b}\right) t+\left(P_{2}-P_{1}\right) w
$$

Once again, the velocity variables can be replaced by the equivalent Mach number expression given by Equation C.6, as shown:

$$
\rho_{1}\left(M_{1} \sqrt{\frac{\gamma P_{1}}{\rho_{1}}}\right)^{2}\left(w-t-\delta^{*}-\theta\right)-\rho_{2}\left(M_{2} \sqrt{\frac{\gamma P_{2}}{\rho_{2}}}\right)^{2} w=\left(P_{1}-P_{b}\right) t+\left(P_{2}-P_{1}\right) w
$$

Evaluating the squared term allows the density variables to be canceled. The expression is then divided by $w$, yielding the following result:

$$
M_{1}^{2} \gamma P_{1}\left(1-\frac{t}{w}-\frac{\delta^{*}}{w}-\frac{\theta}{w}\right)-M_{2}^{2} \gamma P_{2}=\frac{\left(P_{1}-P_{b}\right) t}{w}+P_{2}-P_{1}
$$

The pressure terms can be collected as follows:

$$
P_{1}\left[M_{1}^{2} \gamma\left(1-\frac{t}{w}-\frac{\delta^{*}}{w}-\frac{\theta}{w}\right)+1\right]-P_{2}\left[M_{2}^{2} \gamma+1\right]=\frac{\left(P_{1}-P_{b}\right) t}{w}
$$

In order to obtain the base pressure coefficient on the right-hand side of Equation C.15, the entire expression can be divided by the dynamic pressure at the throat plane, which is defined as:

$$
\frac{1}{2} \rho_{1} V_{1}^{2}
$$


While Equation C.16 is the dynamic pressure for incompressible flow, this definition is still used in the compressible derivation in order to obtain the same base pressure coefficient as the one used by Denton (1993), which is the following:

$$
C_{b, D}=\frac{P_{b}-P_{1}}{\frac{1}{2} \rho V_{1}^{2}}
$$

The dynamic pressure can be recast more appropriately for compressible flow by replacing the velocity with Equation C.6, resulting in the following form:

$$
\frac{1}{2} \gamma M_{1}^{2} P_{1}
$$

Dividing Equation C.15 by the dynamic pressure yields the following, with the base pressure coefficient inserted on the right-hand side:

$$
\frac{P_{1}\left[M_{1}^{2} \gamma\left(1-\frac{t}{w}-\frac{\delta^{*}}{w}-\frac{\theta}{w}\right)+1\right]}{\frac{1}{2} \gamma M_{1}^{2} P_{1}}-\frac{P_{2}\left[M_{2}^{2} \gamma+1\right]}{\frac{1}{2} \gamma M_{1}^{2} P_{1}}=-\frac{C_{b, D} t}{w}
$$

Equation C.19 can be simplified by canceling out the following variables: $\gamma, P_{1}$ and $M_{1}$.

$$
2\left[\left(1-\frac{t}{w}-\frac{\delta^{*}}{w}-\frac{\theta}{w}\right)+\frac{1}{\gamma M_{1}^{2}}\right]-2 \frac{P_{2}}{P_{1}}\left[\frac{M_{2}^{2}}{M_{1}^{2}}+\frac{1}{\gamma M_{1}^{2}}\right]=-\frac{C_{b, D} t}{w}
$$

Equation C.20 can be rearranged as follows to isolate the pressure ratio $\frac{P_{2}}{P_{1}}$.

$$
\frac{P_{2}}{P_{1}}=\frac{1}{\left[\frac{M_{2}^{2}}{M_{1}^{2}}+\frac{1}{\gamma M_{1}^{2}}\right]}\left[\left(1-\frac{t}{w}-\frac{\delta^{*}}{w}-\frac{\theta}{w}\right)+\frac{1}{\gamma M_{1}^{2}}\right]+\frac{C_{b, D} t}{2 w\left[\frac{M_{2}^{2}}{M_{1}^{2}}+\frac{1}{\gamma M_{1}^{2}}\right]}
$$

\section{C.4 Energy Equation}

The energy equation can be written as follows:

$$
\left(h_{1}+\frac{1}{2} V_{1}^{2}\right) \dot{m}_{1}=\left(h_{2}+\frac{1}{2} V_{2}^{2}\right) \dot{m}_{2}
$$


where $h_{1}$ and $h_{2}$ are the enthalpy values at the throat plane and mixed-out plane, respectively. For an ideal gas, the enthalpy can be rewritten as follows:

$$
h=C_{P} T=\left(\frac{\gamma R}{\gamma-1}\right)\left(\frac{P}{\rho R}\right)=\frac{\gamma}{\gamma-1} \frac{P}{\rho}
$$

This definition for the enthalpy can be inserted into Equation C.22, in addition to the expression for velocity in Equation C.6. Additionally, since the mass flow rate into and out of the control volume are the same, the mass flow rates can be canceled, as follows:

$$
\frac{\gamma}{\gamma-1} \frac{P_{1}}{\rho_{1}}+\frac{1}{2}\left(M_{1} \sqrt{\frac{\gamma P_{1}}{\rho_{1}}}\right)^{2}=\frac{\gamma}{\gamma-1} \frac{P_{2}}{\rho_{2}}+\frac{1}{2}\left(M_{2} \sqrt{\frac{\gamma P_{2}}{\rho_{2}}}\right)^{2}
$$

Evaluating the squared terms, the following is obtained:

$$
\frac{\gamma}{\gamma-1} \frac{P_{1}}{\rho_{1}}+\frac{1}{2} \gamma M_{1}^{2} \frac{P_{1}}{\rho_{1}}=\frac{\gamma}{\gamma-1} \frac{P_{2}}{\rho_{2}}+\frac{1}{2} \gamma M_{2}^{2} \frac{P_{2}}{\rho_{2}}
$$

Equation C.25 is multiplied by $\frac{2 \rho_{2}}{\gamma P_{1}}$, and the left-side is factorized, yielding the following expression:

$$
\frac{\rho_{2}}{\rho_{1}}\left[\frac{2}{\gamma-1}+M_{1}^{2}\right]=\frac{2}{\gamma-1} \frac{P_{2}}{P_{1}}+M_{2}^{2} \frac{P_{2}}{P_{1}}
$$

If an inlet Mach number $M_{1}$ is known, the energy equation can be used to solve for the outlet Mach number $M_{2}$. The continuity equation from Equation C.9 is inserted into the energy equation in Equation C.26 in order to eliminate the density ratio $\frac{\rho_{2}}{\rho_{1}}$.

$$
\frac{P_{1}}{P_{2}} \frac{M_{1}^{2}}{M_{2}^{2}} \frac{\left(w-t-\delta^{*}\right)^{2}}{w^{2}}\left[\frac{2}{\gamma-1}+M_{1}^{2}\right]=\frac{2}{\gamma-1} \frac{P_{2}}{P_{1}}+M_{2}^{2} \frac{P_{2}}{P_{1}}
$$

Multiplying Equation C.27 through by $M_{2}^{2}$ and by the pressure ratio $\frac{P_{2}}{P_{1}}$, the following is obtained:

$$
M_{1}^{2} \frac{\left(w-t-\delta^{*}\right)^{2}}{w^{2}}\left[\frac{2}{\gamma-1}+M_{1}^{2}\right]=\frac{2}{\gamma-1}\left(\frac{P_{2}}{P_{1}}\right)^{2} M_{2}^{2}+\left(\frac{P_{2}}{P_{1}}\right)^{2} M_{2}^{4}
$$


Equation C.28 is a second order polynomial in $M_{2}^{2}$ and can be rearranged to show the coefficients A, B and C explicitly, as shown:

$$
\left(M_{2}^{2}\right)^{2}+\frac{2}{\gamma-1}\left(M_{2}^{2}\right)-\left(\frac{P_{1}}{P_{2}}\right)^{2}\left[\frac{2}{\gamma-1}+M_{1}^{2}\right] \frac{M_{1}^{2}\left(w-t-\delta^{*}\right)^{2}}{w^{2}}=0
$$

where the coefficients of the quadratic equation are the following:

$$
\begin{aligned}
& A=1 \\
& B=\frac{2}{\gamma-1} \\
& C=-\left(\frac{P_{1}}{P_{2}}\right)^{2}\left[\frac{2}{\gamma-1}+M_{1}^{2}\right] \frac{M_{1}^{2}\left(w-t-\delta^{*}\right)^{2}}{w^{2}}
\end{aligned}
$$

Equation C.29 can thus be solved using the quadratic equation, as follows:

$$
\left(M_{2}^{2}\right)=\frac{-B \pm \sqrt{B^{2}-4 A C}}{2 A}
$$

\section{C.5 Solution Method}

The end goal of this derivation is to obtain a value for the trailing edge loss coefficient defined Equation C.3 in the introduction. Using the equations derived above, it is possible to obtain values for the pressure ratio $\left(\frac{P_{2}}{P_{1}}\right)$ and the inlet and outlet Mach numbers. The solution to the system of equations requires an iterative process, which can be summarized as follows.

1. Begin with a known value of Mach number at the throat plane $\left(M_{1}\right)$.

2. Assume a value of $M_{2}$. A good starting guess is $M_{2}=M_{1}$.

3. Using Equation C.21, obtain the pressure ratio $\left(\frac{P_{2}}{P_{1}}\right)$.

4. Using Equation C.31 and the correct coefficients A, B and C, obtain a new value for the mixed-out Mach number $\left(M_{2}\right)$.

5. Using the new value of $M_{2}$ as a new guess, return to Step 3 and repeat the remaining steps iteratively. The process can be repeated until the value of mixed-out Mach number 
changes very little with each pass. An exit condition of $\Delta M_{2}<10^{-6}$ was used to produce the figures in this investigation.

6. The pressure ratio $\left(\frac{P_{2}}{P_{1}}\right)$ and the Mach numbers at the throat plane $\left(M_{1}\right)$ and the mixedout plane $\left(M_{2}\right)$ can be inserted into Equation C.3 to obtain the trailing edge loss coefficient for compressible flow. 